\title{
Dietary Patterns and Breast, Colorectal, Lung, and Prostate Cancer: A Systematic Review
}

\section{Dietary Guidelines Advisory Committee, Dietary Patterns Subcommittee \\ Published date: July 15, 2020}

Nutrition Evidence Systematic Review

Center for Nutrition Policy and Promotion

Food and Nutrition Service

U.S. Department of Agriculture

Braddock Metro Center II

1320 Braddock Place

Alexandria, Virginia 22314 
This systematic review was conducted by the 2020 Dietary Guidelines Advisory Committee in collaboration with the Nutrition Evidence Systematic Review (NESR) team at the Center for Nutrition Policy and Promotion, Food and Nutrition Service, U.S. Department of Agriculture (USDA). All systematic reviews from the 2020 Advisory Committee Project are available on the NESR website: https://nesr.usda.gov/2020-dietaryguidelines-advisory-committee-systematic-reviews.

Conclusion statements drawn as part of this systematic review describe the state of science related to the specific question examined. Conclusion statements do not draw implications, and should not be interpreted as dietary guidance. This portfolio provides the complete documentation for this systematic review. A summary of this review is included in the 2020 Advisory Committee's Scientific Report available at www.DietaryGuidelines.gov.

The contents of this document may be used and reprinted without permission. Endorsements by NESR, the Center for Nutrition Policy and Promotion, the Food and Nutrition Service, or the USDA of derivative products developed from this work may not be stated or implied.

Suggested citation for this systematic review: 2020 Dietary Guidelines Advisory Committee and Nutrition Evidence Systematic Review Team. Dietary Patterns and Breast, Colorectal, Lung, and Prostate Cancer: A Systematic Review. 2020 Dietary Guidelines Advisory Committee Project. Alexandria, VA: U.S. Department of Agriculture, Food and Nutrition Service, Center for Nutrition Policy and Promotion, July 2020. Available at: https://nesr.usda.gov/2020-dietary-guidelines-advisory-committee-systematic-reviews.

Related citation: Dietary Guidelines Advisory Committee. 2020. Scientific Report of the 2020 Dietary Guidelines Advisory Committee: Advisory Report to the Secretary of Agriculture and the Secretary of Health and Human Services. U.S. Department of Agriculture, Agricultural Research Service, Washington, DC.

In accordance with Federal civil rights law and USDA civil rights regulations and policies, the USDA, its Agencies, offices, and employees, and institutions participating in or administering USDA programs are prohibited from discriminating based on race, color, national origin, religion, sex, gender identity (including gender expression), sexual orientation, disability, age, marital status, family/parental status, income derived from a public assistance program, political beliefs, or reprisal or retaliation for prior civil rights activity, in any program or activity conducted or funded by USDA (not all bases apply to all programs). Remedies and complaint filing deadlines vary by program or incident.

Persons with disabilities who require alternative means of communication for program information (e.g., Braille, large print, audiotape, American Sign Language, etc.) should contact the responsible Agency or USDA's TARGET Center at (202) 720-2600 (voice and TTY) or contact USDA through the Federal Relay Service at (800) 877-8339. Additionally, program information may be made available in languages other than English.

To file a program discrimination complaint, complete the USDA Program Discrimination Complaint Form, AD3027, found online at How to File a Program Discrimination Complaint and at any USDA office or write a letter addressed to USDA and provide in the letter all of the information requested in the form. To request a copy of the complaint form, call (866) 632-9992. Submit your completed form or letter to USDA by: (1) mail: U.S. Department of Agriculture, Office of the Assistant Secretary for Civil Rights, 1400 Independence Avenue, SW, Washington, D.C. 20250-9410; (2) fax: (202) 690-7442; or (3) email: program.intake@usda.gov.

USDA is an equal opportunity provider, employer, and lender. 


\section{ACKNOWLEDGEMENTS}

\section{Dietary Patterns Subcommittee:}

- Carol Boushey, PhD, MPH, RD, University of Hawaii, Subcommittee Chair

- Jamy Ard, MD, Wake Forest School of Medicine

- Lydia Bazzano, MD, PhD, Tulane University and Ochsner Health System

- Steven Heymsfield, MD, Louisiana State University, Pennington Biomedical Research Center

- Elizabeth Mayer-Davis, PhD, RD, University of North Carolina at Chapel Hill

- Joan Sabaté, DrPH, MD, Loma Linda University

- Linda Snetselaar, PhD, RDN, University of lowa

- Linda Van Horn, PhD, RDN, LD, Northwestern University

- Barbara Schneeman, PhD, University of California, Davis, Chair of the 2020 Dietary Guidelines Advisory Committee

\section{Nutrition Evidence Systematic Review (NESR) Team:}

- Laural Kelly English, PhD, Analyst, Panum Group ${ }^{i}$

- Marlana Bates, MPH, RD, Analyst, Panum Group ${ }^{i}$

- Emily Callahan, MS, Analyst, Office of Nutrition Guidance and Analysis (ONGA), Center for Nutrition Policy and Promotion (CNPP), Food and Nutrition Service (FNS), U.S. Department of Agriculture (USDA)

- Gisela Butera, MLIS, MEd, Systematic Review Librarian, Panum Groupi

- Nancy Terry, MS, MLS, Biomedical Librarian, National Institutes of Health (NIH) Library, U.S. Department of Health and Human Services (HHS)

- Julie Obbagy, PhD, RD, Project lead, ONGA, CNPP, FNS, USDA

\section{Federal Liaisons:}

- Elizabeth Rahavi, RD, ONGA, CNPP, FNS, USDA

- Clarissa (Claire) Brown, MS, MPH, RD, ONGA, CNPP, FNS, USDA

\section{Project Leadership:}

- Eve Essery Stoody, PhD, Designated Federal Officer and Director, ONGA, CNPP, FNS, USDA

- Janet de Jesus, MS, RD, Nutrition Advisor, Office of Disease Prevention and Health Promotion, Office of the Assistant Secretary for Health, HHS

USDA and HHS implemented a process to identify topics and scientific questions to be examined by the 2020 Dietary Guidelines Advisory Committee. The Committee conducted its review of evidence in subcommittees for discussion by the full Committee during its public meetings. The role of the Committee members involved

\footnotetext{
' Under contract with the Food and Nutrition Service, United States Department of Agriculture.
} 
establishing all aspects of the protocol, which presented the plan for how they would examine the scientific evidence, including the inclusion and exclusion criteria; reviewing all studies that met the criteria they set; deliberating on the body of evidence for each question; and writing and grading the conclusion statements to be included in the scientific report the 2020 Committee submitted to USDA and HHS. The NESR team with assistance from Federal Liaisons and Project Leadership, supported the Committee by facilitating, executing, and documenting the work necessary to ensure the reviews were completed in accordance with NESR methodology. More information about the 2020 Dietary Guidelines Advisory Committee, including the process used to identify topics and questions, can be found at www.DietaryGuidelines.gov. More information about NESR can be found at NESR.usda.gov.

The Committee and NESR staff thank USDA's Agricultural Research Service for coordinating the peer review of this systematic review, and the Federal scientist peer reviewers for their time and expertise.

FUNDING SOURCE: United States Department of Agriculture, Food and Nutrition Service, Center for Nutrition Policy and Promotion, Alexandria, VA 


\section{TABLE OF CONTENTS}

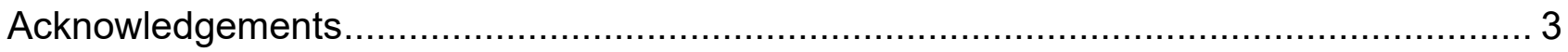

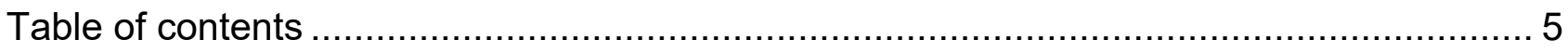

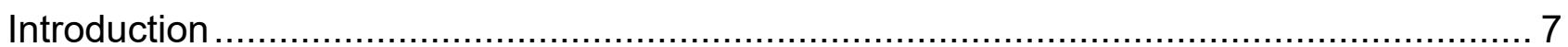

What is the relationship between dietary patterns consumed and risk of certain types of

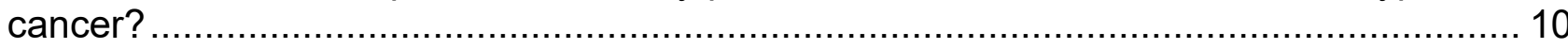

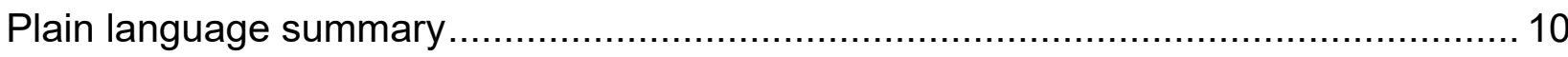

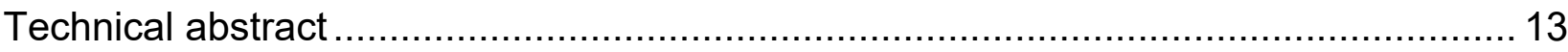

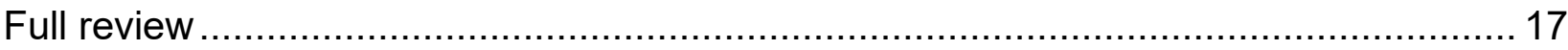

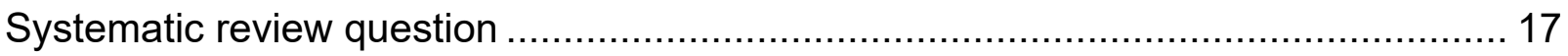

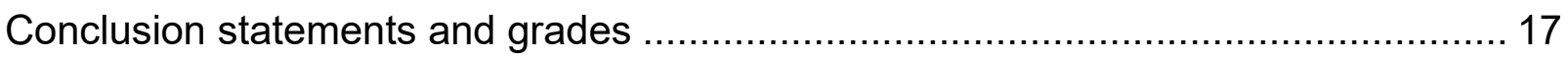

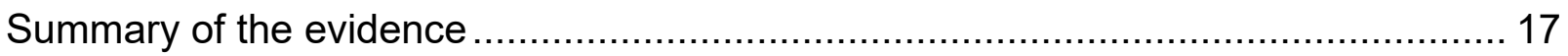

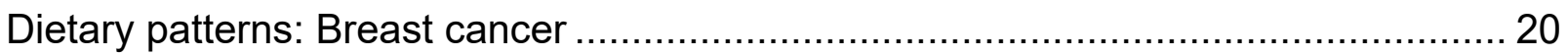

Dietary patterns: Colorectal cancer .............................................................. 27

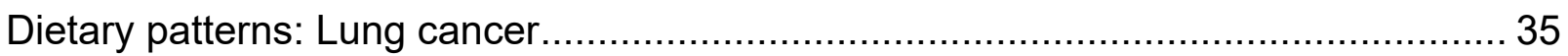

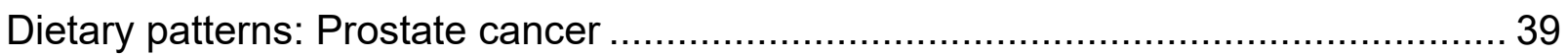

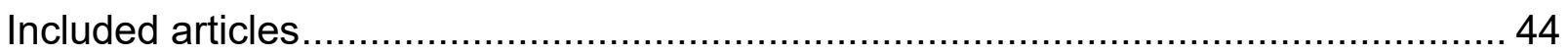

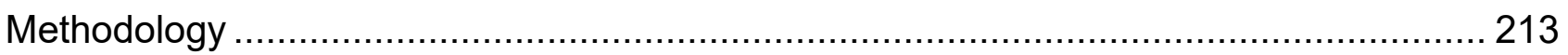

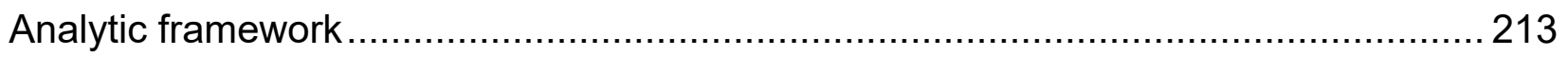

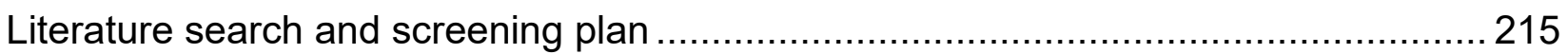

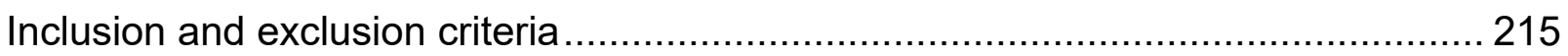

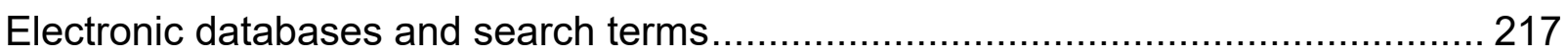

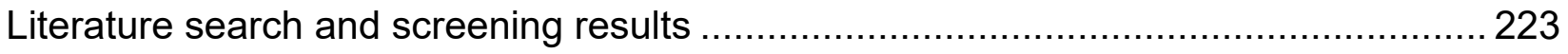

Excluded articles from updated literature search .............................................. 225

Table 1. Description of studies that examined the relationship between dietary patterns and

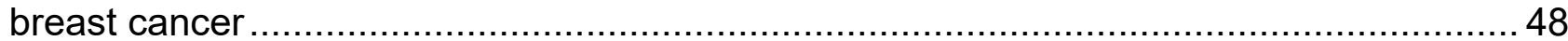

Table 2. Summary of the results from studies that examined the relationship between

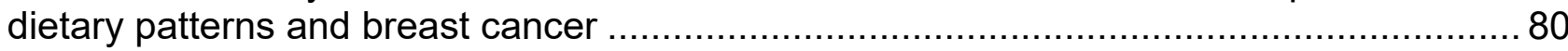
Table 3. Risk of bias for randomized controlled trials examining dietary patterns and breast

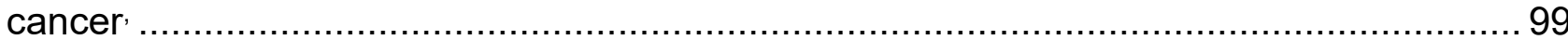

Table 4. Risk of bias for observational studies examining dietary patterns and breast

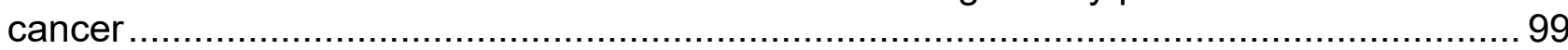

Table 5. Description of studies that examined the relationship between dietary patterns and colorectal cancer. 101

Table 6. Summary of the results from studies that examined the relationship between 
dietary patterns and colorectal cancer

Table 7. Risk of bias for randomized controlled trials examining dietary patterns and colorectal cancer.

Table 8. Risk of bias for observational studies examining dietary patterns and colorectal cancer

Table 9. Description of studies that examined the relationship between dietary patterns and lung cancer 159

Table 10. Summary of the results from studies that examined the relationship between dietary patterns and lung cancer 172 Table 11. Risk of bias for observational studies examining dietary patterns and lung cancer

Table 12. Description of studies that examined the relationship between dietary patterns and prostate cancer 185

Table 13. Summary of the results from studies that examined the relationship between dietary patterns and prostate cancer 202

Table 14. Risk of bias for observational studies examining dietary patterns and prostate cancer, 212

Table 15. Inclusion and exclusion criteria 215

Table 16. Articles excluded after full-text screening with rationale for exclusion

Figure 1: Analytic framework 214

Figure 2: Flow chart of literature search and screening results 


\section{INTRODUCTION}

This document describes a systematic review conducted to answer the following question: What is the relationship between dietary patterns consumed and risk of certain types of cancer? This systematic review was conducted by the 2020 Dietary Guidelines Advisory Committee, supported by USDA's Nutrition Evidence Systematic Review (NESR).

More information about the 2020 Dietary Guidelines Advisory Committee is available at the following website: www.DietaryGuidelines.gov.

NESR specializes in conducting food- and nutrition-related systematic reviews using a rigorous, protocol-driven methodology. More information about NESR is available at the following website: NESR.usda.gov.

NESR's systematic review methodology involves developing a protocol, searching for and selecting studies, extracting data from and assessing the risk of bias of each included study, synthesizing the evidence, developing conclusion statements, grading the evidence underlying the conclusion statements, and recommending future research. A detailed description of the systematic reviews conducted for the 2020 Dietary Guidelines Advisory Committee, including information about methodology, is available on the NESR website: https://nesr.usda.gov/2020-dietary-guidelines-advisory-committee-systematic-reviews. In addition, starting on page 214, this document describes the final protocol as it was applied in the systematic review. A description of and rationale for modifications made to the protocol are described in the 2020 Dietary Guidelines Advisory Committee Report, Part D: Chapter 8. Dietary Patterns.

The systematic review described in this document updates existing systematic reviews that were conducted by the 2015 Dietary Guidelines Advisory Committee with support from USDA's Nutrition Evidence Systematic Review (NESR) team. Information about the 2015 Dietary Guidelines Advisory Committee's review of the evidence on dietary patterns and cancer can be found in their report, which is available at the following website: https://nesr.usda.gov/dietary-patterns-foods-and-nutrients-and-health-outcomessubcommittee and https://www.dietaryguidelines.gov/current-dietary-guidelines/processdevelop-2015-2020-dg/advisory-committee. 


\section{List of abbreviations}

\begin{tabular}{ll}
\hline Abbreviation & Full name \\
\hline AHEI-2010 & Alternative Healthy Eating Index-2010 \\
\hline AICR & American Institute for Cancer Research \\
\hline aMED & alternate Mediterranean diet score \\
\hline aMEDr & alternate Mediterranean diet score without alcohol \\
\hline BMI & Body mass index \\
\hline CSDLH & Canadian Study of Diet, Lifestyle and Health \\
\hline DASH & Dietary Approaches to Stop Hypertension \\
\hline DRE & Digital rectal exam \\
\hline EDIH & Empirical dietary index for hyperinsulinemia \\
\hline EDIP & Empirical dietary inflammatory pattern \\
\hline ERDP & Estrogen-related dietary pattern \\
\hline f/u & Follow-up \\
\hline FSAm-NPS & $\begin{array}{l}\text { Nutrient Profiling System of the British Food Standards Agency } \\
\text { (modified version) }\end{array}$ \\
\hline FSAm-NPS DI & $\begin{array}{l}\text { Nutrient Profiling System of the British Food Standards Agency } \\
\text { dietary index (modified version) }\end{array}$ \\
\hline h & Hour(s) \\
\hline HDI & Human development index \\
\hline HEI-2010 & Healthy Eating Index-2010 \\
\hline HER2 & Human epidermal growth factor receptor 2 \\
\hline HHS & United States Department of Health and Human Services \\
\hline MEDI-LITE & Mediterranean diet score (French NutriNet-Sante study) \\
\hline MET & Metabolic equivalent of task \\
\hline NBSS & Modified Mediterranean Diet \\
\hline
\end{tabular}




\begin{tabular}{ll}
\hline Abbreviation & Full name \\
\hline NESR & Nutrition Evidence Systematic Review \\
\hline NIH & National Institutes of Health \\
\hline PCA & Principal component analysis \\
\hline PCS & Prospective cohort study \\
\hline PNNS-GS & French National Nutrition Health Program-Guideline Score \\
\hline PSA & Prostate-specific antigen \\
\hline RCT & Randomized controlled trial \\
\hline RRR & Reduced rank regression \\
\hline SES & Socioeconomic status \\
\hline USDA & United States Department of Agriculture \\
\hline WCRF & World Cancer Research Fund \\
\hline wk & Week(s) \\
\hline y & Year(s) \\
\hline
\end{tabular}




\section{WHAT IS THE RELATIONSHIP BETWEEN DIETARY PATTERNS CONSUMED AND RISK OF CERTAIN TYPES OF CANCER?}

\section{PLAIN LANGUAGE SUMMARY}

\section{What is the question?}

- The question is: What is the relationship between dietary patterns consumed and risk of certain types of cancer?

\section{What is the answer to the question?}

\section{Dietary patterns: Breast cancer}

- Moderate evidence indicates that dietary patterns rich in vegetables, fruits, and whole grains, and lower in animal-source foods and refined carbohydrates, are associated with reduced risk of postmenopausal breast cancer. The data regarding these dietary patterns and premenopausal breast cancer risk point in the same direction, but the evidence is limited as fewer studies include premenopausal breast cancer.

\section{Dietary patterns: Colorectal cancer}

- Moderate evidence indicates that dietary patterns higher in vegetables, fruits, legumes, whole grains, lean meats and seafood, and low-fat dairy; and low in red and processed meats, saturated fat and sugar-sweetened beverages and sweets relative to other dietary patterns are associated with lower risk of colon and rectal cancer. Moderate evidence also indicates that dietary patterns that are higher in red and processed meats, French fries, potatoes, and sources of sugars (e.g., sugar-sweetened beverages, sweets and dessert foods) are associated with a greater colon and rectal cancer risk.

\section{Dietary patterns: Lung cancer}

- Limited evidence suggests that dietary patterns containing more frequent servings of vegetables, fruits, seafood, grains and cereals, legumes and lean vs. higher fat meats and lower fat or non-fat dairy products may be associated with lower risk of lung cancer, primarily among former smokers and current smokers.

\section{Dietary patterns: Prostate cancer}

- Limited evidence suggests no relationship between dietary patterns and risk of prostate cancer.

Why was this question asked?

- This important public health question was identified by the U.S. Departments of Agriculture (USDA) and Health and Human Services (HHS) to be examined by the 2020 Dietary Guidelines Advisory Committee.

How was this question answered?

- The 2020 Dietary Guidelines Advisory Committee, Dietary Patterns Subcommittee conducted a systematic review to answer this question with support from the 
Nutrition Evidence Systematic Review (NESR) team. The systematic review updates existing systematic reviews conducted by the 2015 Dietary Guidelines Advisory Committee.

- Dietary patterns were defined as the quantities, proportions, variety, or combination of different foods, drinks, and nutrients (when available) in diets, and the frequency with which they are habitually consumed.

\section{What is the population of interest?}

- For the intervention/exposure, children through older adults, age 2 years and older

- For the outcome, children through older adults, age 2 years and older

\section{What evidence was found?}

\section{Dietary patterns: Breast cancer}

- This review identified 26 articles that met inclusion criteria.

- Most studies reported dietary patterns were related with lower risk of postmenopausal breast cancer.

- Dietary patterns were higher in vegetables, fruits, and whole grains, and lower in animal products and refined carbohydrates.

- Alcohol was not consistently included within the dietary patterns

- Few studies reported results for premenopausal breast cancer risk.

- Studies differed in dietary pattern methods, dietary intake assessment, and duration of follow-up.

- Key limitations of the studies include not accounting for key confounders or possible changes in dietary intake over follow-up.

- The 2020 Committee updates and concurs with the conclusions drawn by the 2015 Committee.

\section{Dietary patterns: Colorectal cancer}

- This review identifies 24 articles that met inclusion criteria.

- Most studies reported dietary patterns were related to lower risk of colorectal cancer.

- Dietary patterns were higher in vegetables, fruits, legumes, whole grains, lean meats and seafood, and low-fat dairy, and lower in red and processed meats, saturated fat, sodas, and sweets.

- Alcohol was not consistently included within the dietary patterns.

- Results were more consistent in men, and for total colorectal cancer risk.

- Studies differed in dietary pattern methods, dietary intake assessment, and duration of follow-up.

- Key limitations in the study design and conduct of included articles were identified. This includes a lack of accounting for key confounders or possible changes in dietary intake over follow-up.

- The 2020 Committee updates the conclusions drawn by the 2015 Committee.

\section{Dietary patterns: Lung cancer}

- This review identified 8 articles that met inclusion criteria.

- Most studies reported dietary patterns were associated to lower risk of lung cancer, but had several limitations. 
- Dietary patterns had more frequent servings of vegetables, fruits, seafood, grains and cereals, legumes and lean vs. higher fat meats and lower fat or non-fat dairy products.

- Protective effects of the patterns were more consistent among participants who were former smokers and current smokers than among participants who were never smokers.

- Alcohol was not consistently included within the dietary patterns.

- Many limitations in the study design and conduct of included articles were identified. This includes a lack of accounting for key confounders or possible changes in dietary intake over follow-up.

- The 2020 Committee updates and concurs with the conclusions drawn by the 2015 Committee.

\section{Dietary patterns: Prostate cancer}

- This review identified 8 articles that met inclusion criteria.

- Most studies reported no significant associations between dietary patterns and risk of prostate cancer.

- Many limitations in the study design and conduct of included articles were identified. This includes a lack of accounting for key confounders or possible changes in dietary intake over follow-up.

- The 2020 Committee updates an existing review from the 2015 Committee, which did not draw a conclusion about this relationship.

\section{How up-to-date is this systematic review?}

- This review searched for studies from January, 2014 to January, 2020, and updated existing systematic reviews that included evidence from January, 2000 to January, 2014. 


\section{TECHNICAL ABSTRACT}

\section{Background}

- This important public health question was identified by the U.S. Departments of Agriculture (USDA) and Health and Human Services (HHS) to be examined by the 2020 Dietary Guidelines Advisory Committee.

- The 2020 Dietary Guidelines Advisory Committee, Dietary Patterns Subcommittee conducted a systematic review to answer this question with support from the Nutrition Evidence Systematic Review (NESR) team.

- The goal of this systematic review was to examine the following question: What is the relationship between dietary patterns consumed and risk of certain types of cancer?

\section{Conclusion statements and grades}

\section{Dietary patterns: Breast cancer}

- Moderate evidence indicates that dietary patterns rich in vegetables, fruits, and whole grains, and lower in animal-source foods and refined carbohydrates, are associated with reduced risk of postmenopausal breast cancer. The data regarding these dietary patterns and premenopausal breast cancer risk point in the same direction, but the evidence is limited as fewer studies include premenopausal breast cancer. (Grade: Moderate - Postmenopausal breast cancer risk, Limited Premenopausal breast cancer risk)

\section{Dietary patterns: Colorectal cancer}

- Moderate evidence indicates that dietary patterns higher in vegetables, fruits, legumes, whole grains, lean meats and seafood, and low-fat dairy; and low in red and processed meats, saturated fat and sugar-sweetened beverages and sweets relative to other dietary patterns are associated with lower risk of colon and rectal cancer. Moderate evidence also indicates that dietary patterns that are higher in red and processed meats, French fries, potatoes, and sources of sugars (e.g., sugarsweetened beverages, sweets and dessert foods) are associated with a greater colon and rectal cancer risk. (Grade: Moderate)

\section{Dietary patterns: Lung cancer}

- Limited evidence suggests that dietary patterns containing more frequent servings of vegetables, fruits, seafood, grains and cereals, legumes and lean vs. higher fat meats and lower fat or non-fat dairy products may be associated with lower risk of lung cancer, primarily among former smokers and current smokers. (Grade: Limited)

\section{Dietary patterns: Prostate cancer}

- Limited evidence suggests no relationship between dietary patterns and risk of prostate cancer. (Grade: Limited)

\section{Methods}

- A literature search was conducted using 4 databases (PubMed, Embase, Cochrane, $\mathrm{CINAHL}$ ) to identify articles that evaluated the intervention or exposure of dietary patterns consumed and risk of breast, colorectal, lung, and prostate cancer. A 
manual search was conducted to identify articles that may not have been included in the electronic databases searched. Articles were screened by two NESR analysts independently for inclusion based on pre-determined criteria.

- Data extraction and risk of bias assessment were conducted for each included study, and both were checked for accuracy. The Committee qualitatively synthesized the body of evidence to inform development of a conclusion statement(s), and graded the strength of evidence using pre-established criteria for risk of bias, consistency, directness, precision, and generalizability.

- Dietary patterns were defined as the quantities, proportions, variety, or combination of different foods, drinks, and nutrients (when available) in diets, and the frequency with which they are habitually consumed.

\section{Summary of the evidence}

\section{Dietary patterns: Breast cancer}

- This systematic review update includes 26 studies that examined the relationship between dietary patterns and risk of breast cancer, met inclusion criteria, and were published between January 2014 and January 2020:

- Three studies were randomized controlled trials,

- Twenty-one were prospective cohort studies, and

- Two studies were nested case-control studies.

- The studies were heterogeneous, in terms of which methods were used to identify or assess dietary patterns, how dietary intake was assessed, and duration of followup. However, despite this heterogeneity, the body of evidence was consistent in the types of foods and beverages examined in a number of the patterns, particularly in those studies that reported statistically significant associations with lower risk of breast cancer.

- In a number of studies, dietary patterns that included vegetables, fruits, and whole grains, and that were lower in animal products and refined carbohydrates, were associated with reduced risk of postmenopausal breast cancer.

- Alcohol was not consistently included within the patterns found to be inversely associated with breast cancer risk.

- Few studies reported results for premenopausal breast cancer risk.

- The studies were direct and generalizable, in that the populations, interventions, comparators, and outcomes of interest in the included studies were directly related to the systematic review question, and were applicable to the U.S. population.

- The body of evidence had several risks of bias, particularly in the observational studies, including lack of adjustment for all key confounders, assessment of a dietary pattern only once at baseline or in the first few years of follow-up, and a lack of accounting for possible changes in dietary intake that may have occurred over follow-up.

- This systematic review updates and concurs with the conclusions drawn by the 2015 Committee.

\section{Dietary patterns: Colorectal cancer}

- This systematic review update includes 24 studies that examined the relationship 
between dietary patterns and risk of colorectal cancer, met inclusion criteria, and were published between January 2014 and January 2020:

- Two studies were randomized controlled trials,

- Twenty-one studies were prospective cohort studies, and

- One study was a nested case-control study.

- The studies were heterogeneous, in terms of which methods were used to identify or assess dietary patterns, how dietary intake was assessed, and duration of followup. However, despite this heterogeneity, the body of evidence was consistent in the types of foods and beverages examined in a number of the patterns, particularly in those studies that reported statistically significant associations with lower risk of colorectal cancer.

- In a number of studies, dietary patterns that included vegetables, fruits, legumes, whole grains, lean meats and seafood, and low-fat dairy, and that were lower in red and processed meats, saturated fat, sodas, and sweets were associated with lower risk of colorectal cancer.

- Alcohol was not consistently included within the patterns found to be inversely associated with colorectal cancer risk.

- Results were more consistent in men, and for total colorectal cancer risk.

- The studies were direct and generalizable, in that that the populations, intervention, comparators, and outcomes of interest in the included studies were directly related to the systematic review question and were applicable to the U.S. population.

- The body of evidence had several risks of bias, particularly in the observational studies, including lack of adjustment for all key confounders, assessment of a dietary pattern only once at baseline or in the first few years of follow-up, and a lack of accounting for possible changes in dietary intake that may have occurred over follow-up.

- This systematic review updates the conclusions drawn by the 2015 Committee. The 2020 Committee determined that the body of evidence included in this update was consistent with that considered by the 2015 Committee, with the exception of alcohol. Because alcohol was not consistently part of the patterns found to be significantly associated with lower colorectal cancer risk, and in some cases, were part of cases associated with increased risk, "moderate alcohol" was removed from the conclusion statement.

\section{Dietary patterns: Lung cancer}

- This systematic review update includes 7 prospective cohort studies and one nested case-control study that examined the relationship between dietary patterns and risk of lung cancer, met inclusion criteria, and were published between January 2014 and January 2020.

- Though the body of evidence had some inconsistencies in direction and magnitude of effect, most studies reported significant associations between adherence to a dietary pattern and lower risk of lung cancer.

- In several studies, dietary patterns containing more frequent servings of vegetables, fruits, seafood, grains and cereals, legumes and lean vs. higher fat meats and lower fat or non-fat dairy products were associated with lower risk of lung cancer 
- The protective effects of the patterns were more consistent among participants who were former smokers and current smokers than among participants who were never smokers.

- Alcohol was not consistently included within the patterns found to be inversely associated with lung cancer risk.

- Most studies had large analytic sample sizes with a sufficient number of lung cancer cases occurring over follow-up to examine associations. However, the width of confidence intervals indicates some degree of imprecision within the body of evidence.

- The studies were direct and generalizable, in that the populations, intervention, comparators, and outcomes of interest in the included studies were directly related to the systematic review question, and were applicable to the U.S. population.

- The body of evidence had several risks of bias, including lack of adjustment for all key confounders, assessment of dietary pattern only once at baseline or in the first few years of follow-up, and a lack of accounting for possible changes in dietary intake that may have occurred over follow-up.

- This systematic review updates and concurs with the conclusions drawn by the 2015 Committee.

\section{Dietary patterns: Prostate cancer}

- This systematic review update includes 7 prospective cohort studies and one nested case-control study that examined the relationship between dietary patterns and risk of prostate cancer, met inclusion criteria, and were published between January 2014 and January 2020.

- Though the direction and magnitude of effect across the body of evidence was inconsistent, most studies reported no significant associations between adherence to a dietary pattern and risk of prostate cancer.

- Most studies had large analytic sample sizes with a sufficient number of prostate cancer cases occurring over follow-up to examine associations. However, the width of confidence intervals indicates some degree of imprecision within the body of evidence.

- The studies were direct and generalizable, in that the populations, exposures, comparators, and outcomes of interest in the included studies were directly related to the systematic review question, and were applicable to the U.S. population.

- The body of evidence had several risks of bias, including lack of adjustment for all key confounders, assessment of a dietary pattern only once at baseline or in the first few years of follow-up, and a lack of accounting for possible changes in dietary intake that may have occurred over follow-up.

- This systematic review updates the review done by the 2015 Committee, which did not draw a conclusion regarding the relationship between dietary patterns and the risk of prostate cancer due to limited evidence from a small number of studies with wide variation in study design, dietary assessment methodology and prostate cancer outcome ascertainment. The 2020 Committee determined that, based on the 8 additional studies in their update, limited evidence is now available to suggest no relationship between dietary patterns and risk of prostate cancer. 


\section{FULL REVIEW}

\section{Systematic review question}

What is the relationship between dietary patterns consumed and risk of certain types of cancer?

\section{Conclusion statements and grades}

\section{Dietary patterns: Breast cancer}

Moderate evidence indicates that dietary patterns rich in vegetables, fruits, and whole grains, and lower in animal-source foods and refined carbohydrates, are associated with reduced risk of postmenopausal breast cancer. The data regarding these dietary patterns and premenopausal breast cancer risk point in the same direction, but the evidence is limited as fewer studies include premenopausal breast cancer. (Grade: Moderate - Postmenopausal breast cancer risk, Limited - Premenopausal breast cancer risk)

\section{Dietary patterns: Colorectal cancer}

Moderate evidence indicates that dietary patterns higher in vegetables, fruits, legumes, whole grains, lean meats and seafood, and low-fat dairy; and low in red and processed meats, saturated fat and sugar-sweetened beverages and sweets relative to other dietary patterns are associated with lower risk of colon and rectal cancer. Moderate evidence also indicates that dietary patterns that are higher in red and processed meats, French fries, potatoes, and sources of sugars (e.g., sugar-sweetened beverages, sweets and dessert foods) are associated with a greater colon and rectal cancer risk. (Grade: Moderate)

\section{Dietary patterns: Lung cancer}

Limited evidence suggests that dietary patterns containing more frequent servings of vegetables, fruits, seafood, grains and cereals, legumes and lean vs. higher fat meats and lower fat or non-fat dairy products may be associated with lower risk of lung cancer, primarily among former smokers and current smokers. (Grade: Limited)

\section{Dietary patterns: Prostate cancer}

Limited evidence suggests no relationship between dietary patterns and risk of prostate cancer. (Grade: Limited)

\section{Summary of the evidence}

\section{Dietary patterns: Breast cancer}

- This systematic review update includes 26 studies that examined the relationship between dietary patterns and risk of breast cancer, met inclusion criteria, and were published between January 2014 and January 2020:

- Three studies were randomized controlled trials (RCTs), ${ }^{1-3}$

$\circ$ Twenty-one were prospective cohort studies (PCSs), ${ }^{4-24}$ and 
- Two studies were nested case-control studies. ${ }^{25,26}$

- The studies were heterogeneous, in terms of which methods were used to identify or assess dietary patterns, how dietary intake was assessed, and duration of follow-up ( $\mathrm{f} / \mathrm{u})$. However, despite this heterogeneity, the body of evidence was consistent in the types of foods and beverages examined in a number of the patterns, particularly in those studies that reported statistically significant associations with lower risk of breast cancer.

- In a number of studies, dietary patterns that included vegetables, fruits, and whole grains, and that were lower in animal products and refined carbohydrates, were associated with reduced risk of postmenopausal breast cancer.

- Alcohol was not consistently included within the patterns found to be inversely associated with breast cancer risk.

- Few studies reported results for premenopausal breast cancer risk.

- The studies were direct and generalizable, in that the populations, interventions, comparators, and outcomes of interest in the included studies were directly related to the systematic review question, and were applicable to the U.S. population.

- The body of evidence had several risks of bias, particularly in the observational studies, including lack of adjustment for all key confounders, assessment of a dietary pattern only once at baseline or in the first few years of $f / u$, and a lack of accounting for possible changes in dietary intake that may have occurred over f/u.

- This systematic review updates and concurs with the conclusions drawn by the 2015 Committee.ii

\section{Dietary patterns: Colorectal cancer}

- This systematic review update includes 24 studies that examined the relationship between dietary patterns and risk of colorectal cancer, met inclusion criteria, and were published between January 2014 and January 2020:

- Two studies were RCTs, ${ }^{1,2}$

- Twenty-one studies were PCSs, $, 4,7,16,24,27-43$ and,

- One study was a nested case-control study. ${ }^{44}$

- The studies were heterogeneous, in terms of which methods were used to identify or assess dietary patterns, how dietary intake was assessed, and duration of $\mathrm{f} / \mathrm{u}$. However, despite this heterogeneity, the body of evidence was consistent in the types of foods and beverages examined in a number of the patterns, particularly in those studies that reported statistically significant associations with lower risk of colorectal cancer.

- In a number of studies, dietary patterns that included vegetables, fruits,

\footnotetext{
ii Dietary Guidelines Advisory Committee. Scientific Report of the 2015 Dietary Guidelines Advisory Committee: Advisory Report to the Secretary of Health and Human Services and the Secretary of Agriculture. US Department of Agriculture, Agricultural Research Service. https://health.gov/ourwork/food-nutrition/2015-2020-dietary-guidelines/advisory-report. Published 2015. Accessed April 30, 2020.
} 
legumes, whole grains, lean meats and seafood, and low-fat dairy, and that were lower in red and processed meats, saturated fat, sodas, and sweets were associated with lower risk of colorectal cancer.

- Alcohol was not consistently included within the patterns found to be inversely associated with colorectal cancer risk.

- Results were more consistent in men, and for total colorectal cancer risk.

- The studies were direct and generalizable, in that that the populations, intervention, comparators, and outcomes of interest in the included studies were directly related to the systematic review question and were applicable to the U.S. population.

- The body of evidence had several risks of bias, particularly in the observational studies, including lack of adjustment for all key confounders, assessment of a dietary pattern only once at baseline or in the first few years of $f / u$, and a lack of accounting for possible changes in dietary intake that may have occurred over f/u.

- This systematic review updates the conclusions drawn by the 2015 Committee. The 2020 Committee determined that the body of evidence included in this update was consistent with that considered by the 2015 Committee, with the exception of alcohol. Because alcohol was not consistently part of the patterns found to be significantly associated with lower colorectal cancer risk, and in some cases, were part of cases associated with increased risk, "moderate alcohol" was removed from the conclusion statement.

\section{Dietary patterns: Lung cancer}

- This systematic review update includes 7 PCSs ${ }^{4,14,24,27,45-47}$ and one nested case-control study ${ }^{48}$ that examined the relationship between dietary patterns and risk of lung cancer, met inclusion criteria, and were published between January 2014 and January 2020.

- Though the body of evidence had some inconsistencies in direction and magnitude of effect, most studies reported significant associations between adherence to a dietary pattern and lower risk of lung cancer.

- In several studies, dietary patterns containing more frequent servings of vegetables, fruits, seafood, grains and cereals, legumes and lean vs. higher fat meats and lower fat or non-fat dairy products were associated with lower risk of lung cancer

- The protective effects of the patterns were more consistent among participants who were former smokers and current smokers than among participants who were never smokers.

- Alcohol was not consistently included within the patterns found to be inversely associated with lung cancer risk.

- Most studies had large analytic sample sizes with a sufficient number of lung cancer cases occurring over f/u to examine associations. However, the width of confidence intervals indicates some degree of imprecision within the body of evidence.

- The studies were direct and generalizable, in that the populations, intervention, comparators, and outcomes of interest in the included studies were directly 
related to the systematic review question, and were applicable to the U.S. population.

- The body of evidence had several risks of bias, including lack of adjustment for all key confounders, assessment of dietary pattern only once at baseline or in the first few years of $f / u$, and a lack of accounting for possible changes in dietary intake that may have occurred over $\mathrm{f} / \mathrm{u}$.

- This systematic review updates and concurs with the conclusions drawn by the 2015 Committee.

\section{Dietary patterns: Prostate cancer}

- This systematic review update includes 7 PCSs $4,6,7,14,16,49,50$ and one nested case-control study ${ }^{51}$ that examined the relationship between dietary patterns and risk of prostate cancer, met inclusion criteria, and were published between January 2014 and January 2020.

- Though the direction and magnitude of effect across the body of evidence was inconsistent, most studies reported no significant associations between adherence to a dietary pattern and risk of prostate cancer.

- Most studies had large analytic sample sizes with a sufficient number of prostate cancer cases occurring over f/u to examine associations. However, the width of confidence intervals indicates some degree of imprecision within the body of evidence.

- The studies were direct and generalizable, in that the populations, exposures, comparators, and outcomes of interest in the included studies were directly related to the systematic review question, and were applicable to the U.S. population.

- The body of evidence had several risks of bias, including lack of adjustment for all key confounders, assessment of a dietary pattern only once at baseline or in the first few years of $f / u$, and a lack of accounting for possible changes in dietary intake that may have occurred over $\mathrm{f} / \mathrm{u}$.

- This systematic review updates the review done by the 2015 Committee, which did not draw a conclusion regarding the relationship between dietary patterns and the risk of prostate cancer due to limited evidence from a small number of studies with wide variation in study design, dietary assessment methodology and prostate cancer outcome ascertainment. The 2020 Committee determined that, based on the 8 additional studies in their update, limited evidence is now available to suggest no relationship between dietary patterns and risk of prostate cancer.

\section{Dietary patterns: Breast cancer}

\section{Description of the evidence}

This systematic review update includes 26 studies that examined the relationship between dietary patterns and risk of breast cancer, met inclusion criteria, and were published between January 2014 and January 2020. (Table 1). Three studies are randomized controlled trials (RCTs), ${ }^{1-3} 21$ are prospective cohort studies, ${ }^{4-24}$ and 2 are 
nested case-control studies. ${ }^{25,26}$

\section{Population/participant characteristics}

The studies included in this systematic review were conducted in Canada, ${ }^{25}$ France ${ }^{5-}$ $7,14,16$ Japan, ${ }^{15,22}$ Spain, ${ }^{3}$ Sweden, ${ }^{11,17}$ The Netherlands, ${ }^{23,24}$ the United Kingdom, ${ }^{26}$ the United States, ${ }^{1,2,8-10,12,13,19-21}$, and Europe (Denmark, France, Germany, Greece, Italy, the Netherlands, Norway, Spain, Sweden, United Kingdom). ${ }^{4,18}$

Two studies reported results from the same RCT, ${ }^{1,2}$ but reported results at different lengths of $\mathrm{f} / \mathrm{u}$. Four studies are from the same cohort in France, 5,7,14,16 2 were from the same European cohort, 4,18 and 2 each were from 2 U.S. cohorts ${ }^{9,21,12,13}$ but examined different dietary patterns in relation to breast cancer risk.

Sample sizes of the studies were large, ranging from 2,492 to 330,766 participants. Studies enrolled women, and mean age of participants at baseline ranged from approximately 38 years to 64 years. Mean BMI ranged from $\sim 21$ to $28 \mathrm{~kg} / \mathrm{m}^{2}$.

All studies excluded participants with prevalent or prior history of cancer, and some excluded participants who were diagnosed with cancer during the first 2 or $3 y$ of f/u. ${ }^{4,6,7}$ Studies included participants who were healthy and/or at risk of chronic disease, or diagnosed with a chronic disease other than cancer. One study excluded participants who had a history of stroke, cardiovascular disease, or diabetes at baseline. ${ }^{15}$

\section{Intervention/exposure}

Included studies examined dietary patterns using a variety of methods (Table 1). Two RCTs tested an intervention with increased vegetables, fruits, and grains, and decreased carbohydrates, and total, saturated, and unsaturated fat, ${ }^{1,2}$ and another tested an intervention of a Mediterranean diet with extra-virgin olive oil, with nuts, or with decreased fat intakes. ${ }^{3}$ Fourteen observational studies examined adherence to dietary patterns using different indices or scores. ${ }^{4-7,10,12,14,16-19,21,23,24,26}$ Five studies identified dietary patterns using factor analysis $12,15,22,25,26$ and 5 studies identified dietary patterns using reduced rank regression. ${ }^{8,9,11,13,26}$ One study examined variations of vegetarian diets. ${ }^{20}$

Dietary intake was assessed using a variety of validated dietary assessment methods, including food frequency questionnaires, 24-hour dietary recalls, or dietary records. Most studies assessed diet once, at baseline, and 9 studies collected dietary data at baseline and at least one other time during $f / u^{1-3,5-7,14,16,19}$.

\section{Outcome assessment}

All included studies examined risk of developing breast cancer, with f/u ranging from $4 y$ to $23 y$. Most studies reported risk of total breast cancer. Nine studies examined risk of premenopausal breast cancer, $5,7,12-15,17,22,2513$ studies examined risk of postmenopausal breast cancer, $5,7,9-11,14,15,17,18,20,22,25,26$ and 5 studies examined risk of invasive breast cancer. ${ }^{1,2,8,9,21}$

\section{Evidence synthesis}

Results from the included articles in this systematic review are provided in Table 1 and Table 2. Prentice et $\mathrm{al}^{1}$ and Thomson et $\mathrm{al}^{2}$ reported results from the Women's Health 
Initiative Dietary Modification Trial, conducted in the United States. Both reported no difference between intervention and comparison groups in risk of total or invasive breast cancer during f/u, through $19.6 \mathrm{y}$. However, Thomson $\mathrm{et}^{2} \mathrm{l}^{2}$ did report that women with higher baseline fat intake (quartiles) had significantly reduced risk of invasive breast cancer.

Toledo et $\mathrm{al}^{3}$ reported results from the Prevención con Dieta Mediterránea (PREDIMED) trial in Spain. They found that breast cancer risk after $4.8 \mathrm{y}$ f/u was significantly reduced after consuming a Mediterranean diet supplemented with extravirgin olive oil compared to a control low-fat diet.

Catsburg et $\mathrm{al}^{25}$ reported results from 2 Canadian cohorts, the Canadian Study of Diet, Lifestyle and Health (CSDLH), and the National Breast Screening Study (NBSS).

Higher adherence to the Meat and potatoes pattern was associated with increased risk of postmenopausal breast cancer in both the CSDLH and NBSS cohorts. However, the Meat and potatoes pattern was not associated with total risk of breast cancer or risk of premenopausal breast cancer in either cohort. Higher adherence to the Healthy pattern in the CSDLH cohort at 60y was associated with decreased risk of breast cancer after $13 \mathrm{y} / \mathrm{u}$. However, results were not significant when pre- and postmenopausal women were analyzed separately. In addition, there was no significant association between adherence to the healthy pattern at 40-59y in the NBSS cohort and risk of breast cancer after 23y f/u. Adherence to the Ethnic pattern was not significantly associated with risk of breast cancer in either the CSDLH or NBSS cohort.

Deschasaux et al, ${ }^{5}$ Deschasaux et al, ${ }^{4}$ and Donnenfeld et al ${ }^{6}$ examined the Nutrient Profiling System of the British Food Standards Agency (modified version) (FSAm-NPS) score and breast cancer risk in different cohorts, from France and several European countries. Deschausaux et al ${ }^{4}$ found that consuming a diet that scores higher on the FSAm-NPS at $51 \mathrm{y}$ was associated with increased risk of breast cancer after $15.3 \mathrm{y} f / \mathrm{u}$. Deschausaux et $\mathrm{al}^{5}$ found that higher FSAm-NPS score at $51 \mathrm{y}$ was significantly associated with increased risk of total and premenopausal breast cancer after $4 \mathrm{y}$ f/u. However, FSAm-NPS score was not significantly associated with risk of postmenopausal breast cancer. Finally, Donnenfeld et $\mathrm{al}^{6}$ reported that FSAm-NPS score at 49y was not significantly associated with risk of breast cancer after 12.6y f/u.

Fiolet et $\mathrm{al}^{7}$ examined an ultra-processed food score in a French cohort and found that higher ultra-processed food score at $49 \mathrm{y}$ was significantly associated with increased risk of postmenopausal breast cancer after $5.4 \mathrm{y} f / \mathrm{u}$. The higher score, when analyzed continuously, was also associated with higher risk of total breast cancer. However, ultra-processed food score at $49 y$ was not significantly associated with risk of total breast cancer after $5.4 \mathrm{y} f / \mathrm{u}$, when analyzed categorically. It was also not associated with risk of premenopausal breast cancer (categorically or continuously).

Guinter et $\mathrm{al}^{8}$ and Guinter et $\mathrm{al}^{9}$ examined an estrogen-related dietary pattern (ERDP), derived using reduced rank regression, in 2 different cohorts from the United States. Guinter et al ${ }^{8}$ found that higher ERDP score at $62 \mathrm{y}$ was significantly associated with increased risk of total and invasive breast cancer after 10.9y f/u. However, Guinter et $\mathrm{al}^{9}$ reported that ERDP score at 58y was not significantly associated with risk of total 
postmenopausal or invasive breast cancer after $6-12 \mathrm{y} / \mathrm{u}$. Harris et al ${ }^{11}$ examined a different estrogen dietary pattern, also derived using reduced rank regression, and reported that higher adherence to an estrogen dietary pattern at 62y was significantly associated with increased risk of breast cancer after $15 \mathrm{y} / \mathrm{u}$.

Haridass et $\mathrm{al}^{10}$ examined several different dietary pattern indices/scores in a U.S. cohort, and found that aMED, DASH, AHEI-2010, and Paleo scores at 40y were not significantly associated with risk of postmenopausal breast cancer risk after $\sim 14 \mathrm{y}$ f/u.

Harris et al ${ }^{12}$ and Harris et al $^{13}$ examined several different dietary patterns in the same cohort of women from the U.S. Harris et $\mathrm{al}^{12}$ found that, in all women, higher adherence to the 'Prudent' pattern during adolescence was significantly associated with reduced risk of breast cancer after 22y f/u. However, there was no significant association with risk of premenopausal breast cancer risk. In addition, adherence to the 'Western', 'Fast food', and AHEI patterns during adolescence were not significantly associated with risk of total or premenopausal breast cancer risk after 22y f/u. Harris et $\mathrm{al}^{13}$ found that higher adherence to an inflammatory dietary pattern during adolescence was significantly associated with increased risk of premenopausal breast cancer after $22 \mathrm{f} / \mathrm{u}$. However, adherence to the inflammatory dietary pattern was not significantly associated with total or postmenopausal breast cancer risk. Results were similar when the early adulthood inflammatory pattern was analyzed, and when adolescent and early adult inflammatory patterns were averaged, except for the results for all cases of breast cancer. When the inflammatory dietary pattern was calculated based on an average of adolescence and young adulthood, higher adherence was significantly associated with increased risk of total breast cancer after $22 \mathrm{y} f / \mathrm{u}$.

Kane-Diallo et al ${ }^{14}$ found that a pro plant-based dietary score at $49 y$, in a French cohort, was not significantly associated with risk of breast cancer, including pre- and postmenopausal breast cancer, after $4.3 \mathrm{y} / \mathrm{u}$.

Kojima et $\mathrm{al}^{15}$ examined dietary patterns in a Japanese cohort, and found that a higher animal food pattern adherence at 55y was significantly associated with increased premenopausal breast cancer risk after $16.9 \mathrm{y} / \mathrm{u}$. However, animal food pattern adherence was not significantly associated with postmenopausal breast cancer risk. In addition, adherence to the vegetable pattern or the dairy product pattern at 55y were not significantly associated with postmenopausal breast cancer risk after 16.9y f/u.

Lavalette et $\mathrm{al}^{16}$ found that, in a French cohort of women, scores representing AHEI2010, Mediterranean diet score (MEDI-LITE), and French National Nutrition Health Program-Guideline Score (PNNS-GS) at 49y were not significantly associated with risk of breast cancer after $8.5 \mathrm{y} / \mathrm{u}$.

Li et $\mathrm{al}^{17}$ examined a cohort of Swedish women, and found the Healthy Nordic food index score at $39 y$ was not significantly associated with risk of breast cancer (total, pre- and post-menopausal) after $20 \mathrm{y} / \mathrm{u}$.

McKenzie et al ${ }^{18}$ derived and examined a diet score in a large European cohort, reporting that a higher diet score at $53 y$ was significantly associated with reduced risk of postmenopausal breast cancer after $10.9 \mathrm{y} / \mathrm{u}$.

Nomura et al ${ }^{19}$ examined women in a cohort from the U.S., and found that adherence 
to the World Cancer Research Fund/American Institute for Cancer Research (WCRF/AICR) diet score at 38y was not significantly associated with risk of breast cancer after $13.9 \mathrm{y}$ f/u when analyzed categorically or continuously. In addition, adherence to the WCRF/AICR diet score was also not significantly associated with breast cancer risk when analyzed categorically, time-varying. However, a higher WCRF/AICR score, diet only, time-varying, analyzed continuously, was associated with significantly decreased risk of breast cancer after $13.9 \mathrm{y} / \mathrm{u}$.

In a cohort from the United States, Penniecook-Sawyers et a ${ }^{20}$ examined four different vegetarian dietary patterns compared to a non-vegetarian dietary pattern. Results showed that adherence to the various vegetarian patterns at $64 \mathrm{y}$ were not significantly associated with risk of total or premenopausal breast cancer after $7.8 \mathrm{y} / \mathrm{u}$.

Petimar et al ${ }^{21}$ examined several different dietary patterns scores in a U.S. cohort, and found that a higher DASH score at $55 \mathrm{y}$ was significantly associated with reduced risk of total invasive breast cancer after 7.6y f/u. AHEI-2010 and aMED scores, with and without alcohol, at 55y, were not significantly associated with risk of total invasive breast cancer after 7.6y f/u.

Pot et $\mathrm{al}^{26}$ examined several dietary patterns, dervied using different methodologies, in a case-control study using cases and controls from 4 cohorts within the United Kingdom. The Medieterranean diet score; a principal component analysis (PCA) identifying three dietary patterns; and a reduced rank regression (RRR) informed by response variables alcohol, total fat, and fiber were created. Using RRR, a high response score for alcohol was associated with a higher risk of total or postmenopausal breast cancer.

Shin et al ${ }^{22}$ identified several dietary patterns using factor analysis in a Japanese cohort. Higher adherence to a Westernised dietary pattern at 57y was significantly associated with increased risk of breast cancer after 14.6y f/u. When pre- and postmenopausal breast cancer were analyzed separately, only post-menopausal breast cancer risk was significantly associated with Westernized dietary pattern adherence. Adherence to a prudent or traditional Japanese dietary pattern at 57y was not significantly associated with risk of breast cancer after 14.6y f/u. Results were similar when pre- and post-menopausal breast cancer were analyzed separately.

Van den Brandt et al ${ }^{23}$ examined several dietary patterns scores in a cohort from the Netherlands. Alternative Mediterranean Diet (aMED) and mMED scores, with and without alcohol, were not significantly associated with risk of breast cancer after 20.3y $\mathrm{f} / \mathrm{u}$. Results were also not significant when stratified by years of f/u, age at baseline, smoking status, alcohol intake, BMI, physical activity, and family history of breast cancer. Also, aMED vs. WCRF, diet only, scores were not significantly associated with breast cancer risk.

Voortman et a ${ }^{24}$ examined women from three sub-cohorts of the Rotterdam Study, and found the Dutch Dietary Guidelines 2015 score at 64y was not significantly associated with breast cancer after $11 \mathrm{y}$ f/u. 


\section{Assessment of the evidence ${ }^{i i i}$}

This systematic review update included 26 studies that met inclusion criteria, including 3 RCTs and 23 observational studies. The studies were heterogeneous, both in terms of the methods used to identify or assess dietary patterns, how dietary intake was assessed, and in duration of $\mathrm{f} / \mathrm{u}$. However, despite this heterogeneity there was consistency in the types of foods and beverages examined in a number of the patterns, particularly in those studies that reported statistically significant associations with lower risk of breast cancer. In a number of studies, dietary patterns that included vegetables, fruits, and whole grains, and were lower in animal products and refined carbohydrate, were associated with reduced risk of post-menopausal breast cancer. Fewer studies reported results for pre-menopausal breast cancer risk.

Publication bias is always a consideration, however it is not a serious concern for this body of evidence because a mix of significant and non-significant findings were reported.

As outlined and described below, the body of evidence examining dietary patterns and risk of breast cancer was assessed for the following elements used when grading the strength of evidence.

- Risk of bias: The included studies had a number of potential risks of bias, or limitations that may have influenced study results (Table 1; Table 3; Table 4). While observational studies accounted for a number of potential confounders, they did not adjust for all key confounders, such as race/ethnicity, hormonal contraceptive use, or socioeconomic status. All studies, regardless of study design, examined dietary patterns once at baseline or in the first few years of f/u, and did not account for possible changes in dietary intake that may have occurred over $\mathrm{f} / \mathrm{u}$. In addition, the studies enrolled older individuals, and did not account for dietary patterns consumed earlier in life. None of the studies fully accounted for and/or analyzed the impact of missing data, either due to loss to f/u or criteria used when selecting participants into the analyses. Finally, because preregistered statistical plans are uncommon for observational studies, the risk of potential selective outcome reporting is unclear.

- Consistency: Though there were some inconsistencies in the direction and magnitude of effect across the body of evidence, most studies reporting significant results found that dietary patterns including vegetables, fruits and whole grains, and were lower in animal products and refined carbohydrate, were associated with reduced risk of breast cancer. Results were primarily reported for risk of postmenopausal breast cancer risk, as fewer studies examined premenopausal breast cancer.

\footnotetext{
iiiA detailed description of the methodology used for grading the strength of the evidence is available on the NESR website: https://nesr.usda.gov/2020-dietary-guidelines-advisory-committee-systematicreviews and in Part C of the following reference: Dietary Guidelines Advisory Committee. 2020. Scientific Report of the 2020 Dietary Guidelines Advisory Committee: Advisory Report to the Secretary of Agriculture and the Secretary of Health and Human Services. U.S. Department of Agriculture, Agricultural Research Service, Washington, DC.
} 
- Directness: The populations, intervention or exposures, comparators, and outcomes of interest in the included studies are directly related to the systematic review question.

- Precision: Though the included studies did not report power analyses or sample size calculations, the majority had large analytic sample sizes with a sufficient number of breast cancer cases, particularly postmenopausal breast cancer cases, occurring over f/u to examine associations. However, there were fewer cases of premenopausal breast cancer. The width of confidence intervals indicates some degree of imprecision within the body of evidence.

- Generalizability: The study participants, interventions and/or exposures, comparators, and outcomes examined in the body of evidence are applicable to the U.S. population.

This systematic review updates and concurs with the conclusion drawn by the 2015 Dietary Guidelines Advisory Committee. iv The 2015 Committee concluded that, "Moderate evidence indicates that dietary patterns rich in vegetables, fruits and whole grains, and lower in animal products and refined carbohydrate, are associated with reduced risk of postmenopausal breast cancer. The data regarding this dietary pattern and premenopausal breast cancer risk point in the same direction, but the evidence is limited due to fewer studies." The 2015 conclusion was based on 26 articles, including $1 \mathrm{RCT}$, and 25 prospective cohort studies, that were published between January 2000 and January 2014. The 2020 Committee determined that the body of evidence included in this update was consistent with that considered by the 2015 Committee.

\section{Research recommendations}

The 2020 Committee concurs with the 2015 Committee that to better assess the relationship between dietary patterns and risk of developing breast cancer, additional research is needed to:

1. Improve and validate novel dietary assessment tools for the accurate assessment of dietary patterns over the life course, including the use of biomarkers

2. Adopt methodologic approaches for defining different dietary patterns such that patterns can be consistently identified, scored and compared across studies

- Assess associations of vegetarian diet patterns, particularly vegan diets and risk of breast cancer

- Examine the relationship of highly processed food patterns with breast cancer risk

3. Establish population studies starting earlier in life to better capture dietary

\footnotetext{
iv Dietary Guidelines Advisory Committee. Scientific Report of the 2015 Dietary Guidelines Advisory Committee: Advisory Report to the Secretary of Health and Human Services and the Secretary of Agriculture. US Department of Agriculture, Agricultural Research Service. https://health.gov/ourwork/food-nutrition/2015-2020-dietary-guidelines/advisory-report. Published 2015. Accessed April 30, 2020.
} 
patterns contributing to risk of breast cancer risk later in life. Important considerations would be addressing phases of the life cycle relevant to breast cancer, including childhood and menarche, adolescence and periods of mammary gland development and growth, periods of reproduction and lactation and subsequent years prior to cancer development

4. Assess associations of dietary patterns by subtypes of breast cancer defined by histopathologic outcomes, tumor hormone receptor status, molecular genotypes, gene expression patterns and other biological characteristics that influence the tumor behavior, for example, by tumor hormone receptor status and other relevant phenotypic characteristics (e.g. HER2 status)

5. Examine how anthropometry, physical activity, sedentary behaviors, and sleep modify the relationship between dietary patterns and risk of breast cancer.

6. Examine the impact of SES and ethnic/racial groups regarding dietary patterns and breast cancer.

\section{Dietary patterns: Colorectal cancer}

\section{Description of the evidence}

This systematic review update includes 24 articles, including 2 articles from one randomized controlled trial, ${ }^{1,2}$ and 21 prospective cohort studies, , ,7,16,24,27-43 and one nested case-control study ${ }^{44}$ that examined the relationship between dietary patterns and risk of colorectal cancer, met inclusion criteria, and were published between January 2014 and January 2020 (Table 5).

\section{Population/participant characteristics}

The randomized controlled trial included in this systematic review ${ }^{1,2}$ was conducted among 48,835 postmenopausal women, mean age $\sim 62 \mathrm{y}$, from the United States. The studies reported risk of colorectal cancer at different f/u time points (13.5y and 19.6y).

The observational studies included in this systematic review were conducted in France, Japan, Sweden, The Netherlands, the United Kingdom, the United States, and a multi-country study from Europe (Denmark, France, Germany, Greece, Italy, the Netherlands, Norway, Spain, Sweden, United Kingdom). Two studies are from the same cohort in France ("NutriNet-Sante"), ${ }^{7,16}$ but examined different dietary patterns in relation to colorectal cancer risk. Five studies were from the same cohorts in the United States (Nurses' Health Study, Health Professionals F/u Study), ${ }^{31,32,35,38,39}$ four of which examined different dietary patterns.

Sample sizes of the studies were large, ranging from 8,050 to 471,495 participants. Most studies enrolled women and men, with the mean age of participants at baseline ranging from approximately 39 years to $64 \mathrm{y}$. Four studies enrolled women only. ${ }^{29,36,41,43}$ Mean BMI of participants ranged from approximately 24 to $28 \mathrm{~kg} / \mathrm{m}^{2}$.

The health status of study participants enrolled in the studies was representative of the general population, including healthy individuals, as well as those at-risk for or diagnosed with a chronic disease. All studies excluded participants with prevalent or prior history of cancer, and some excluded participants who were diagnosed with 
cancer during the first 1-2y of f/u. 4,7,29,41 In addition, several studies excluded individuals with a history of ulcerative colitis, ${ }^{31,32,35,38}$ and one study excluded participants with end-stage renal disease, a history of colorectal polyps, or a firstdegree relative with colon cancer. ${ }^{40}$

\section{Intervention/exposure}

Included studies examined dietary patterns using a variety of methods (Table 5). The randomized controlled trial compared a low-fat diet with fruits, vegetables, and grains to a control diet. Most studies examined adherence to dietary patterns using different indices or scores, 4,7,16,24,27-29,34-36,38-44. Three studies identified dietary patterns using factor or cluster analysis ${ }^{30,32,37}$ one study used reduced rank regression to derive dietary patterns, ${ }^{31}$ and one study examined variations of vegetarian diets. ${ }^{33}$

Dietary intake was assessed using a variety of validated dietary assessment methods, including food frequency questionnaires, 24-hour dietary recalls, or dietary records. Most studies assessed diet once, at baseline, though several studies collected dietary data on 2 or more occasions. ${ }^{7,16,27,31,32,35,38,39}$

\section{Outcome assessment}

All included studies examined risk of developing colorectal cancer, with f/u ranging from $4 y$ to 20y. All studies reported risk of overall colorectal cancer.

Additionally 4 studies also examined risk of rectal and colon cancer separately, ${ }^{30,33,41,42}$ and 8 others further examined rectal colon, proximal colon, and distal colon separately. ${ }^{29,32,34,35,37-39,44}$

\section{Evidence synthesis}

\section{Randomized controlled trials}

Thomson et $\mathrm{al}^{2}$ and Prentice et $\mathrm{al}^{1}$ reported results from the Women's Health Initiative Dietary Modification Trial in the United States (Table 5 and Table 6). The trial compared a low-fat diet, higher in fruits, vegetables, and whole and total grains, to a control diet, and reported no significant group differences in risk of colorectal cancer during the intervention, and after all f/u time points (8.5y, 13.5y, and 19.6y).

\section{Observational studies}

\section{Mediterranean diet scores}

A number of observational studies examined adherence to a Mediterranean diet, using various different indices and scores, and found that higher adherence was associated with decreased risk, especially among men (Table 5 and Table 6). Fasanelli et al ${ }^{28}$ reported that higher adherence to the Italian Mediterranean Index at 50y was significantly associated with decreased risk of colorectal cancer after $11 \mathrm{y} \mathrm{f/u}$, and the reported association was independent of waist-to-hip ratio. Jones et al ${ }^{29}$ found that higher adherence to the Mediterranean diet score at 52y was significantly associated with lower risk of colorectal and rectal cancer after $17.4 \mathrm{y}$ f/u. However, the Mediterranean diet score at 52y was not significantly associated with colon, proximal colon, or distal colon cancer. Park et $\mathrm{al}^{34}$ reported that higher adherence to a Mediterranean diet at $60 \mathrm{y}$ was significantly associated with lower risk of colorectal cancer after $16 \mathrm{y}$ f/u in men, but not women. And, when results were stratified by race, 
greater adherence was significantly associated with lower risk of colorectal cancer in all groups, except African Americans. Results from Petimar et $\mathrm{al}^{35}$ showed that higher adherence to a Mediterranean diet at 55y was significantly associated with lower risk of rectal cancer after $26 \mathrm{y} f / u$. When women and men were analyzed separately, results were significant in men, but not in women. In addition, higher adherence in men was also associated with lower risk of total colorectal cancer. However, adherence in men and women was not significantly associated with different types of colorectal cancer (colorectal, colon, distal colon, proximal colon). Schulpen and van den Brandt ${ }^{44}$ found the Mediterranean diet score, with and without alcohol, at 61y was not significantly associated with colorectal cancer risk after $20.3 \mathrm{y}$ f/u. Results were also not significant when stratified by smoking status in men, alcohol consumption, body mass index, education, or family history of colorectal cancer. However, in women former smokers, greater adherence to the aMEDr was significantly associated with lower risk of colorectal cancer. Torres Stone et $\mathrm{al}^{40}$ also examined a Mediterranean diet score and found that higher scores were significantly associated with lower risk of colorectal cancer after 123 months $\mathrm{f} / \mathrm{u}$ in men, but not in women.

Several studies examined adherence to a Mediterranean diet, and reported no significant associations with risk of colorectal cancer. Boden et $\mathrm{al}^{27}$ and Lavalette et $\mathrm{al}^{16}$ both found the Mediterranean diet score was not significantly associated with risk of colorectal cancer after 15y and 8.5y of f/u in men and women. And, both Cheng et $\mathrm{al}^{43}$ and Vargas et $\mathrm{al}^{41}$ examined data from cohort of women-only, and found that Mediterranean diet score was not significantly associated with risk of colorectal cancer after $18.1 \mathrm{y}$ and $12.4 \mathrm{y}$ of f/u, respectively.

\section{DASH diet score}

All studies that examined adherence to the DASH diet, reported statistically significant associations showing that higher adherence was associated with lower risk of colorectal cancer (Table 5 and Table 6). Park et al ${ }^{34}$ found that in both men and women, higher DASH adherence was associated with lower risk of colorectal cancer after $16 \mathrm{y} / \mathrm{u}$. Higher adherence was also associated with significantly lower risk of left colon and rectum cancer, but not right colon cancer. And, when results were stratified by race, increased adherence was significantly associated with lower risk of colorectal cancer in all groups, except African Americans. Petimar et al ${ }^{35}$ found that while DASH score at 55y was not significantly associated with colorectal cancer (colorectal, colon, distal colon, proximal colon, rectal cancer) after $26 \mathrm{y} f / \mathrm{u}$, in men, greater DASH adherence was significantly associated with lower total colorectal, total colon, and distal colon cancer risk. Results from Torres Stone et $\mathrm{al}^{40}$ showed that in both men and women, higher DASH score was significantly associated with lower risk of colorectal cancer after 123 months f/u. Additionally, Vargas et a ${ }^{41}$ reported that higher DASH scores at $63 y$ were significantly associated with decreased risk of colorectal and colon cancer after $12.4 \mathrm{y} / \mathrm{u}$, but not rectal cancer.

\section{Dietary guidelines-related scores}

Several studies examined adherence to the Dietary Guidelines for Americans, using either the Healthy Eating Index 2010 (HEl-2010) or the Alternative Healthy Eating Index 2010 (AHEl-2010) (Table 5 and Table 6)). Results consistently showed that 
higher HEI-2010 scores were associated with lower risk of colorectal cancer. However, results for the AHEI-2010 were less consistent, particularly among women. Park et al ${ }^{34}$ examined both the Healthy Eating Index 2010 (HEI-2010) and the alternative HEI2010. In men, increased adherence to the HEI-2010 and AHEI-2010 at 60y were significantly associated with lower risk of colorectal cancer after 16y f/u. In women, greater adherence to the HEI-2010, but not the AHEI-2010, was significantly associated with lower risk of colorectal cancer. HEI-2010 and AHEI-2010 scores were also associated with significantly lower risk of left colon and rectum cancer, but not right colon cancer. Furthermore, when results were stratified by race, increased adherence to both scores was significantly associated with lower risk of colorectal cancer in all groups, except African Americans. Vargas et al ${ }^{41}$ also examined both the $\mathrm{HEI}-2010$ and the AHEI-2010 in data from a cohort of women, and found that higher $\mathrm{HEI}-2010$ score at $63 \mathrm{y}$ was significantly associated with decreased risk of colorectal and colon cancer after $12.4 \mathrm{y} / \mathrm{u}$, but not rectal cancer. But, AHEl-2010 score was not significantly associated with risk of colorectal, colon, or rectal cancer. Torres Stone et $\mathrm{al}^{40}$ examined the HEI-2010 and found that in both men and women, higher HEI-2010 scores were significantly associated with lower risk of colorectal cancer after 123 months f/u. Petimar et al ${ }^{35}$ reported that AHEI-2010 score at 55y, in men and women pooled, was not significantly associated with colorectal cancer (colorectal, colon, distal colon, proximal colon, rectal cancer) after 26y f/u. However, in men only, higher AHEI2010 adherence was significantly associated with lower total colorectal cancer risk. Finally, Lavalette et al ${ }^{16}$ found that AHEI-2010 score at $49 y$ was not significantly associated with risk of colorectal cancer.

\section{Other indices and scores}

A variety of additional indices and scores were also examined in this body of evidence (Table 5 and Table 6), and those results are described below.

Some studies reported that higher adherence to various scores was associated with lower risk of colorectal cancer. Liu et $\mathrm{al}^{31}$ found that higher empirical dietary inflammatory pattern score at $52 \mathrm{y}$ was associated with significantly greater risk of colorectal cancer, but this was only significant for quintile 2. Comparisons between the rest of the quintiles were not significant. Lavalette et $\mathrm{al}^{16}$ found that higher French National Nutrition Health Program-Guideline Score (PNNS-GS) when analyzed continuously (but not categorically) at $49 y$ was associated with significantly lower risk of colorectal cancer after $8.5 \mathrm{y} f / \mathrm{u}$. Voortman et al ${ }^{24}$ found that higher Dutch Dietary Guidelines 2015 score at $64 \mathrm{y}$ was significantly associated with lower risk of colorectal cancer after $11 \mathrm{y}$ f/u. Additionally, Vulcan et $\mathrm{al}^{42}$ reported that higher colorectal diet quality index score at $59 \mathrm{y}$ was significantly associated with lower risk of colorectal, colon, and rectal cancer after f/u.

Some studies reported no significant associations between various scores and risk of colorectal cancer. Cheng et al ${ }^{43}$ found the evolutionary-concordance diet score at $61 \mathrm{y}$ was not associated with risk of colorectal cancer over a 18y period of f/u. Fiolet et al ${ }^{7}$ reported the ultra-processed food score at $49 \mathrm{y}$ was not significantly associated with risk of colorectal cancer after $5.4 \mathrm{y} / \mathrm{u}$. Results from Roswall et $\mathrm{al}^{36}$ showed that the Healthy Nordic Food Index score at 39y was not significantly associated with risk of 
colorectal cancer during f/u. Schulpen and van den Brandt ${ }^{44}$ found that WCRF/AICR scores, with and without alcohol, at $61 \mathrm{y}$ was not significantly associated with colorectal cancer risk after $20.3 \mathrm{y} / \mathrm{u}$. Results were also not significant when stratified by smoking status in men, alcohol consumption, body mass index, education, or family history of colorectal cancer.

Finally, some studies reported that higher adherence to various scores emphasizing less healthful foods was associated with increased risk of colorectal cancer. Deschasaux et $\mathrm{al}^{4}$ reported that consuming a diet that scores higher on the Nutrient Profiling System of the British Food Standards Agency (modified version) (FSAm-NPS) at $51 \mathrm{y}$ was associated with a higher risk of colorectal cancer after $15.3 \mathrm{y}$ of f/u. Tabung et $\mathrm{al}^{38}$ found that higher adherence to EDIP scores for up to $26 \mathrm{y}$ of $\mathrm{f} / \mathrm{u}$, which represented a proinflammatory diet, was significantly associated with risk of colorectal cancer, colon cancer, proximal colon cancer, distal colon cancer in men, women, and both combined, as well as risk of rectal cancer in men. Results were not significant for rectal cancer risk in women or men/women combined. In addition, when stratified by $\mathrm{BMI}<$ or $>25 \mathrm{~kg} / \mathrm{m}^{2}$, results were significant for men in both groups, and only in women with $\mathrm{BMl}<25 \mathrm{~kg} / \mathrm{m}^{2}$. When stratified by alcohol intake (no drink, $0.1-1$ drink $/$ day, $>1$ drink/d), results were significant for men consuming no drinks or 0.1-1 drink/day, and in women consuming no drinks. Tabung et $\mathrm{al}^{39}$ also found that the higher empirical dietary index for hyperinsulinemia (EDIH) scores at $55 \mathrm{y}$ were significantly associated with higher risk of colorectal cancer after $26 \mathrm{y} / \mathrm{u}$. Results were similar for men only, women only, and men and women combined for colorectal, colon, and distal colon cancer. Results were also significant for women only and for men and women combined for proximal colon cancer, and in men only for rectal cancer. However, EDIH scores were not significantly associated with proximal colon cancer in men only, or in rectal cancer in women only or men and women combined. When stratified by BMI < or $>25 \mathrm{~kg} / \mathrm{m}^{2}$, results were significant for men in both groups, and only in women with $\mathrm{BM}>25 \mathrm{~kg} / \mathrm{m}^{2}$. When results were stratified by physical activity (MET-hour/wk below/above median), results were significant for men and women below the median.

\section{Other dietary patterns}

Three studies examined dietary patterns that were identified using factor or cluster analysis (Table 5 and Table 6). Kumagai et al ${ }^{30}$ found that higher adherence to a highdairy, high-fruit-and-vegetable, low, alcohol dietary pattern at $60 \mathrm{y}$ was significantly associated with lower risk of colorectal and rectal cancer after 11y f/u. The Japanese dietary pattern and animal food dietary patterns were not significantly associated with risk of colorectal cancer. In addition, none of the patterns were significantly associated with risk of colon or rectal cancer, analyzed as separate outcomes. Mehta et $\mathrm{al}^{32}$ found that higher adherence to a prudent dietary pattern at 52y was significantly associated with lower risk of colorectal cancer after $32 \mathrm{y} / \mathrm{u}$. However, it was not significantly associated with proximal colon, distal colon, or rectal cancer. Results for the prudent dietary pattern were similar in women; however, in men, higher prudent diet score was significantly associated with decreased risk of distal colon and rectal cancer. Conversely, higher adherence to a western dietary pattern was shown to be significantly associated with increased risk of colorectal, distal colon, and rectal 
cancer. However, it was not significantly associated with proximal colon cancer. And, results for the western dietary pattern were similar when men and women were analyzed separately. Results from Shin et $\mathrm{al}^{37}$ also showed that higher adherence to a prudent dietary pattern was significantly associated with decreased risk of colorectal cancer and distal cancer in men and increased risk of rectal cancer in women. And, higher adherence to a westernized dietary pattern was significantly associated with increased risk of colon cancer and distal cancer in women.

Finally, one study, Orlich et $\mathrm{al}^{33}$, examined various types of vegetarian diets (Table 5 and Table 6). Results showed that consuming a vegetarian vs. nonvegetarian diet at $58 y$ was associated with a significant reduction in risk of colorectal cancer after 7.3y $\mathrm{f} / \mathrm{u}$. When results were broken down by type of vegetarian diet, consuming a pescovegetarian diet vs a nonvegetarian diet at 58y was associated with lower risk, while there were no differences with vegan, lacto-ovo, or semi-vegetarian diets. When results were stratified by sex and race, they were no significant associations in men or Black participants. However, in women and non-black vegetarians, there was a borderline significant lower risk of colorectal cancer. And, vegetarian diet at 58y was not significantly associated with risk of rectal or colon cancer after $7.3 \mathrm{y} / \mathrm{u}$, when they were analyzed separately.

\section{Assessment of the evidence ${ }^{v}$}

This systematic review update includes 24 articles, including 2 articles from one RCT and 21 prospective cohort studies. The studies were heterogeneous, both in terms of which methods were used to identify or assess dietary patterns, how dietary intake was assessed, and in duration of $f / u$. However, despite this heterogeneity, the body of evidence was consistent in the types of foods and beverages examined in a number of the patterns, particularly in those studies reporting statistically significant associations with lower risk of colorectal cancer. In a number of studies, dietary patterns that included vegetables, fruits, legumes, whole grains, lean meats and seafood, and lowfat dairy, and were lower in red and processed meats, saturated fat, sodas, and sweets were associated with lower risk of colorectal cancer. While alcohol was included in many of the dietary patterns, there was inconsistency in how it was treated within the patterns (e.g., a positive, neutral, or negative contributor for various indices and scores) and in analyses (e.g., analysis of scores with or without alcohol, stratification by alcohol intake).

Publication bias is always a consideration, however it is not a serious concern for this body of evidence because a mix of significant and non-significant findings were reported.

As outlined and described below, the body of evidence examining dietary patterns and

\footnotetext{
${ }^{v} \mathrm{~A}$ detailed description of the methodology used for grading the strength of the evidence is available on the NESR website: https://nesr.usda.gov/2020-dietary-guidelines-advisory-committee-systematicreviews and in Part C of the following reference: Dietary Guidelines Advisory Committee. 2020. Scientific Report of the 2020 Dietary Guidelines Advisory Committee: Advisory Report to the Secretary of Agriculture and the Secretary of Health and Human Services. U.S. Department of Agriculture, Agricultural Research Service, Washington, DC.
} 
risk of colorectal cancer was assessed for the following elements used when grading the strength of evidence.

- Risk of bias: The included studies had a number of potential risks of bias, or limitations that may have influenced study results (Table 5; Table 7; Table 8). While observational studies adjusted for a number of potential confounders, they did not account for all key confounders, such as race/ethnicity, inflammatory bowel disease, and colorectal polyps. All studies, regardless of study design, examined dietary patterns once at baseline or in the first few years of f/u and did not account for possible changes in dietary intake that may have occurred over $\mathrm{f} / \mathrm{u}$. In addition, the studies enrolled older individuals, and did not account for dietary patterns consumed earlier in life. None of the studies fully accounted for and/or analyzed the impact of missing data, either due to loss to f/u or criteria used when selecting participants into the analyses. Finally, because preregistered statistical plans are uncommon for observational studies, the risk of potential selective outcome reporting is unclear.

- Consistency: Though there were some inconsistencies in the direction and magnitude of effect across the body of evidence, most studies that reported significant associations found that dietary patterns that included vegetables, fruits, legumes, whole grains, lean meats and seafood, and low-fat dairy, and were lower in red and processed meats, saturated fat, sodas, and sweets were associated with lower risk of colorectal cancer. Alcohol was not consistently included within the patterns found to be inversely associated with colorectal cancer risk. Results were most consistent in men, and for total colorectal cancer risk.

- Directness: The populations, interventions and/or exposure, comparators, and outcomes of interest in the included studies are directly related to the systematic review question.

- Precision: Though the included studies did not report power analyses or sample size calculations, the majority had large analytic sample sizes with a sufficient number of colorectal cancer cases. However, there were fewer cases of colon, rectal, proximal colon, and distal colon cancer. The width of confidence intervals indicates some degree of imprecision within the body of evidence.

- Generalizability: The study participants, interventions and/or exposures, comparators, and outcomes examined in the body of evidence are applicable to the U.S. population.

This systematic review updates the conclusion drawn by the 2015 Dietary Guidelines Advisory Committee. ${ }^{\text {vi }}$ The 2015 Advisory Committee concluded that, "Moderate

\footnotetext{
vi Dietary Guidelines Advisory Committee. Scientific Report of the 2015 Dietary Guidelines Advisory Committee: Advisory Report to the Secretary of Health and Human Services and the Secretary of Agriculture. US Department of Agriculture, Agricultural Research Service. https://health.gov/ourwork/food-nutrition/2015-2020-dietary-guidelines/advisory-report. Published 2015. Accessed April 30,
} 
evidence indicates an inverse association between dietary patterns that are higher in vegetables, fruits, legumes, whole grains, lean meats and seafood, low-fat dairy and moderate alcohol; and low in red and processed meats, saturated fat and sodas and sweets relative to other dietary patterns and the risk of colon and rectal cancer. Conversely, diets that are higher in red and processed meats, French fries and potatoes, and sources of sugars (e.g., sugar-sweetened beverages, sweets and dessert foods) are associated with a greater colon and rectal cancer risk." The 2015 conclusion was based on 22 articles, including $1 \mathrm{RCT}$, and 21 prospective cohort studies, that were published between January, 2000 and January, 2014. The 2020 Dietary Guidelines Advisory Committee determined that the body of evidence included in this update was consistent with that considered by the 2015 Committee, with the exception of alcohol. Because alcohol was not consistently part of the patterns found to be significantly associated with lower colorectal cancer risk, and in some cases, were part of cases associated with increased risk, "moderate alcohol" was removed from the conclusion statement.

\section{Research recommendations}

The 2020 Committee concurs with the 2015 Committee that in order to better assess the relationship between dietary patterns and risk of developing colorectal cancer, additional research is needed to:

1. Improve and validate dietary assessment tools for the accurate assessment of dietary patterns over the life course, include the use of biomarkers

2. Adopt methodologic approaches for defining different dietary patterns such that patterns can be consistently identified, scored and compared across studies. However, recognize exploratory pattern definitions and analysis can assist with discovery especially as food supply changes

3. Establish cohort studies that start earlier in life in order to capture dietary patterns contributing to risk of colorectal cancer risk later in life

4. Examine the impact of gender, SES and ethnic/racial groups regarding dietary patterns and colorectal cancer

5. Examine dietary patterns in context of physical activity and sedentary behaviors

6. Continue to explore the role of energy balance and obesity (including patterns of weight change throughout the life cycle) and anthropometric measures in colorectal cancer risk

7. Assess associations of dietary patterns by sub-types of colorectal cancer defined by location within the colon, cancer genetics and other histopathologic characteristics

- Assess associations of vegetarian dietary patterns and risk of colorectal cancer

- Examine the relationship of highly processed food patterns with breast cancer risk

8. Continue to define the role of specific nutrients, phytochemicals and foods that may individually or in combination contribute to risk of colorectal cancer 
9. Examine the interactives roles of dietary intake and the gut microbiome and its influence on colorectal cancer risk.

\section{Dietary patterns: Lung cancer}

\section{Description of the evidence}

This systematic review update includes 7 prospective cohort studies ${ }^{5,14,24,27,45-47}$ and one nested case-control study ${ }^{48}$ that examined the relationship between dietary patterns and risk of lung cancer, met inclusion criteria, and were published between January 2014 and January 2020 (Table 9).

\section{Population/participant characteristics}

The studies included in this systematic review were conducted in Australia, ${ }^{46} \mathrm{France}^{14}$ Italy, ${ }^{47}$ the Netherlands, ${ }^{24,48}$ Sweden, ${ }^{27}$ the United States, ${ }^{45}$ and Europe (Denmark, France, Germany, Greece, Italy, the Netherlands, Norway, Spain, Sweden, United Kingdom). ${ }^{4}$ Sample sizes of the studies were large, ranging from 4,336 to 460,700 participants. Studies enrolled older adults, mean age of participants at baseline ranged from approximately 51 years to 64 years. Mean BMI was $\sim 24-27 \mathrm{~kg} / \mathrm{m}^{2}$.

All studies excluded participants with prevalent or prior history of cancer, and some excluded participants who were diagnosed with cancer during the first $1 \mathrm{y}$ to $3 \mathrm{y}$ of f/u. $., 24,27$ Studies included participants who were healthy and/or at risk of chronic disease, or diagnosed with a chronic disease other than cancer. One study excluded all participants who had a history of or existing diabetes, heart attack, or angina at baseline ${ }^{46}$ and another excluded participants with end-stage renal disease ${ }^{45}$

\section{Intervention/exposure}

All 8 studies examined adherence to dietary patterns using indices or scores (Table 9; Table 10). $5,14,24,27,45-48$ However, the studies differed in terms of the indices or scores assessed.

Dietary intake was assessed using a variety of validated dietary assessment methods, including food-frequency questionnaires, 24-hour dietary recalls, or dietary records. Most studies assessed diet once, at baseline, though 2 studies assessed diet at multiple time points during the first $1-2$ years of $f / u .^{14,27}$

\section{Outcome assessment}

All included studies examined risk of developing lung cancer, with f/u ranging from $4 y$ to $20 \mathrm{y}$. All studies reported risk of total lung cancer.

\section{Evidence synthesis}

Results reported in the studies included in this systematic review were mixed (Table 9; Table 10). In a large cohort from the United States, Anic et al ${ }^{45}$ found that higher adherence to the HEI-2010, AHEI-2010, aMED, and DASH scores at $62 \mathrm{y}$ were all associated with significantly lower risk of lung cancer after $10.5 \mathrm{y} / \mathrm{u}$. When they analyzed by smoking status, AHEI-2010, aMED, and DASH score results remained significant for former smokers, but were no longer significant for never or current 
smokers. For HEl-2010, results remained significant for former and current smokers, but were no longer significant for never smokers.

Maissoneuve et $\mathrm{al}^{47}$ also examined adherence to the aMED, in an Italian cohort, and reported that a higher aMED score at $>50 \mathrm{y}$ was associated with significantly lower risk of lung cancer after $8.5 \mathrm{y} / \mathrm{u}$. Hodge et $\mathrm{al}^{46}$ examined a different Mediterranean diet score in an Australian cohort, reporting that a higher score at 40-69y was associated with significantly lower risk of lung cancer after $18 \mathrm{y} / \mathrm{u}$. When they analyzed by smoking status, results remained significant in current smokers, but were no longer significant in never and former smokers. However, Boden et $\mathrm{al}^{27}$ examined a Mediterranean diet score in a Swedish cohort at $46 \mathrm{y}$ and found no significant association with risk of lung cancer after $15 \mathrm{y} / \mathrm{u}$.

Schulpen and van den Brandt ${ }^{48}$ implemented a nested case-cohort approach in the Netherlands, and examined several different dietary patterns scores, including 2 variations on Mediterranean diet scores, including the aMED, in relation to risk of lung cancer. Schulpen and van den Brandt ${ }^{48}$ analyzed all diet scores with and without alcohol. Results showed that aMED and mMED, and WCRF/AICR scores, with and without alcohol, at $61 \mathrm{y}$ were not significantly associated with lung cancer after $20.3 \mathrm{y}$ $\mathrm{f} / \mathrm{u}$. Though, in men, WCRF/AICR score with alcohol was significantly associated with lower risk of lung cancer.

Deschasaux et $\mathrm{al}^{4}$ reported that in a group of European men, score on the Nutrient Profiling System of the British Food Standards Agency dietary index (modified version) (FSAm-NPS DI) at 51y was not significantly associated with risk of lung cancer after 15.3y f/u in men and women combined, or in women-only. However, in men only, higher FSAm-NPS score was significantly associated with increased risk of lung cancer.

Kane-Diallo et al $^{14}$ found that a higher pro plant-based dietary score, among a group of French participants who were 57y at baseline, was associated with a significantly reduced risk of lung cancer after $4.3 \mathrm{y} / \mathrm{u}$.

Voortman et $\mathrm{al}^{24}$ applied the Dutch Dietary Guidelines 2015 score to inform disease incidence among men and women at $64 \mathrm{y}$. With regard to lung cancer, after $11 \mathrm{y}$ f/u in a group of participants from the Netherlands, adherence to the guidelines was not significantly associated with lung cancer.

\section{Assessment of the evidence ${ }^{\text {vi }}$}

This systematic review update included 8 studies that met inclusion criteria, and all were observational studies. The studies varied in terms of which dietary patterns were examined, how dietary intake was assessed, and in duration of f/u. However, despite

\footnotetext{
viiA detailed description of the methodology used for grading the strength of the evidence is available on the NESR website: https://nesr.usda.gov/2020-dietary-guidelines-advisory-committee-systematicreviews and in Part $\mathrm{C}$ of the following reference: Dietary Guidelines Advisory Committee. 2020. Scientific Report of the 2020 Dietary Guidelines Advisory Committee: Advisory Report to the Secretary of Agriculture and the Secretary of Health and Human Services. U.S. Department of Agriculture, Agricultural Research Service, Washington, DC.
} 
this heterogeneity, most studies reported significant associations between higher adherence to a dietary pattern higher in fruits, vegetables, whole grains, fish, and legumes, and lower in red and processed meat and dairy products and lower risk of lung cancer, particularly among participants who were current or former smokers, and therefore at higher risk of lung cancer. Many studies adjusted for smoking status, including factors such as current or former smoking status, duration of smoking, or cigarettes per day. Other stratified analyses by smoking status, reported different results in former or current smokers compared to never smokers. However, while it is apparent that smoking status may modify the effect of dietary patterns on risk of lung cancer, it unclear whether there may be residual confounding or reverse causality that may be impacting the results.

Publication bias is always a consideration, however it is not a serious concern for this body of evidence because a mix of significant and non-significant findings were reported.

As outlined and described below, the body of evidence examining dietary patterns and risk of lung cancer was assessed for the following elements used when grading the strength of evidence.

- Risk of bias: The included studies had a number of potential risks of bias, or limitations that may have impacted study results (Table 9 and Table 11). While studies adjusted for a number of potential confounders, including smoking, they did not adjust for all key confounders, such as race/ethnicity, history of lung disease, or environmental exposure to lung carcinogens. All studies examined dietary patterns once at baseline or in the first few years of $\mathrm{f} / \mathrm{u}$, and did not account for possible changes in dietary intake that may have occurred over f/u. In addition, the studies enrolled older individuals, and did not account for dietary patterns consumed earlier in life. None of the studies fully accounted for and/or analyzed the impact of missing data, either due to lost to f/u or criteria used when selecting participants into the analyses. Finally, because preregistered statistical plans were not available for some of the included articles, the risk of potential selective outcome reporting is unclear.

- Consistency: Though there were some inconsistencies in the direction and magnitude of effect across the body of evidence, most studies reported significant associations between higher adherence to a dietary pattern higher in fruits, vegetables, whole grains, fish, and legumes, and lower in red and processed meat and dairy products and lower risk of lung cancer. Most studies had large analytic sample sizes with a sufficient number of prostate cancer cases occurring over f/u to examine associations. However, the width of confidence intervals indicates some degree of imprecision within the body of evidence.

- Directness: The populations, interventions and/or exposures, comparators, and outcomes of interest in the included studies are directly related to the systematic review question. 
- Precision: Though the included studies did not report power analyses or sample size calculations, the majority had large analytic sample sizes with a sufficient number of lung cancer cases occurring over $\mathrm{f} / \mathrm{u}$ to examine associations. However, the width of confidence intervals indicates some degree of imprecision within the body of evidence.

- Generalizability: The study participants, interventions and/or exposures, comparators, and outcomes examined in the body of evidence are applicable to the U.S. population.

This systematic review updates and concurs with the conclusion drawn by the 2015 Dietary Guidelines Advisory Committee. viii The 2015 Advisory Committee concluded that, "Limited evidence from a small number of studies suggests a lower risk of lung cancer associated with dietary patterns containing more frequent servings of vegetables, fruits, seafood, grains and cereals, legumes and lean vs. higher fat meats and lower fat or non-fat dairy products. Despite reported modest significant reductions in risk, definitive conclusions cannot be established at this time because of the small number of articles, as well as wide variation in study design, dietary assessment and case ascertainment." The 2015 conclusion was based on three prospective cohort studies published between January 2000 and January 2014. The 2020 Dietary Guidelines Advisory Committee determined that the 8 additional studies in this update are consistent with the 2015 conclusion.

\section{Research recommendations}

The 2020 Committee concurs with the 2015 Committee that in order to better assess the relationship between dietary patterns and risk of developing lung cancer, additional research is needed to:

1. Examine dietary patterns and associations with lung cancer risk among diverse ethnic/racial minority groups in the United States

2. Investigate how dietary patterns consumed across the life cycle, including children and in younger aged smokers impact the risk of lung cancer

3. Examine dietary patterns in association with smoking status (including smokers, ex-smokers, never smokers and passive smoking exposure) and by duration and amount of smoking with risk of lung cancer including possible biological mechanisms

4. Examine alternatives to tobacco smoking and/or vaping, and their associated with dietary intakes, body weight, and with risk of lung cancer

5. Consider histopathologic and molecular subtypes of lung cancer and whether dietary pattern and lung cancer association vary by subtype

6. Continue to define the role of specific nutrients, phytochemicals and foods that

viii Dietary Guidelines Advisory Committee. Scientific Report of the 2015 Dietary Guidelines Advisory Committee: Advisory Report to the Secretary of Health and Human Services and the Secretary of Agriculture. US Department of Agriculture, Agricultural Research Service. https://health.gov/ourwork/food-nutrition/2015-2020-dietary-guidelines/advisory-report. Published 2015. Accessed April 30, 2020. 
may individually or in combination during various stages of the life cycle impact the risk of lung cancer.

\section{Dietary patterns: Prostate cancer}

\section{Description of the evidence}

This systematic review update includes 7 prospective cohort studies ${ }^{5-7,14,16,49,50}$ and one nested case-control study ${ }^{51}$ that examined the relationship between dietary patterns and risk of prostate cancer, met inclusion criteria, and were published between January 2014 and January 2020 (Table 12).

\section{Population/participant characteristics}

The studies included in this systematic review were conducted in France, ${ }^{6,7,14,16}$ Japan, 49 the Netherlands, ${ }^{51}$ the United States, ${ }^{50}$ and Europe (Denmark, France, Germany, Greece, Italy, the Netherlands, Norway, Spain, Sweden, United Kingdom). ${ }^{4}$ Three studies are from the same cohort in France, $, 714,16$ but examined different dietary patterns in relation to prostate cancer risk. Sample sizes of the studies were large, ranging from 2,753 to 140,729 participants. Studies enrolled older men and mean age of participants at baseline ranged from approximately 43 y to $61 \mathrm{y}$. Mean BMI was $\sim 24-$ $25 \mathrm{~kg} / \mathrm{m}^{2}$.

All studies excluded participants with prevalent or prior history of cancer, and some excluded participants who were diagnosed with cancer during the first 2 or $3 y$ of $f / u .4,6,7$ Studies included participants who were healthy and/or at risk of chronic disease or diagnosed with a chronic disease other than cancer.

\section{Intervention/exposure}

Included studies examined dietary patterns using a variety of methods (Table 12). Six studies examined adherence to dietary patterns using different indices or scores. ${ }^{4,6,7,14,16,51}$ One study identified dietary patterns using factor analysis ${ }^{49}$ and one study examined variations of vegetarian diets. ${ }^{50}$

Dietary intake was assessed using a variety of validated dietary assessment methods, including food-frequency questionnaires and dietary records. Most studies assessed diet once, at baseline, though the 3 studies from one cohort in France collected dietary data using at least 3 dietary records collected at different specified times during the first $1-2$ years of $f / u .^{7,14,16}$

\section{Outcome assessment}

All included studies examined risk of developing prostate cancer, with $\mathrm{f} / \mathrm{u}$ ranging from $4 y$ to $20 y$. All studies reported risk of overall prostate cancer. Additionally, three studies also examined risk of advanced prostate cancer, ${ }^{49-51}$ one examined nonadvanced prostate cancer, ${ }^{51}$ and one examined localized prostate cancer. ${ }^{49}$

\section{Evidence synthesis}


Results reported in the studies included in this systematic review were mixed, though many reported no significant associations between dietary patterns and risk of lung cancer (Table 12; Table 13). Deschasaux et al ${ }^{4}$ reported that European men who consumed a diet that scored higher on the Nutrient Profiling System of the British Food Standards Agency dietary index (modified version) (FSAm-NPS DI) at 51y, had borderline significantly higher risk of prostate cancer after 15.3y f/u. However, Donnenfeld et $\mathrm{al}^{6}$ found that FSAm-NPS DI score, in a cohort of French men, $\sim 49 \mathrm{y}$ at baseline, was not significantly associated with risk of prostate cancer after $12.6 \mathrm{y} \mathrm{f/u}$.

All three studies from the same cohort in France, reported no significant relationships between various dietary patterns and risk of prostate cancer. Fiolet et $\mathrm{al}^{7}$ found that an ultra-processed food score at 49y was not significantly associated with risk of prostate cancer after $5.4 \mathrm{y} \mathrm{f} / \mathrm{u}$. Kane-Diallo et al $^{14}$ found that a pro plant-based dietary score at $57 \mathrm{y}$ was not significantly associated with risk of prostate cancer after $4.3 \mathrm{y} / \mathrm{u}$. And, Lavalette et al $^{16}$ found that AHEI-2010, MEDI-LITE, and PNNS-GS scores at 55y were not significantly associated with risk of prostate cancer after $8.5 \mathrm{y} / \mathrm{u}$.

Schulpen and van den Brandt ${ }^{51}$ conducted a nested case-control study in the Netherlands, and examined several different dietary patterns scores, with and without alcohol, in relation to risk of total, nonadvanced, and advanced prostate cancer. Higher alternative Mediterranean Diet Score (aMED) score (continuous and categorical) with and without alcohol was associated with significantly increased risk of nonadvanced prostate cancer after 20.3y f/u. In addition, the aMED score (continuous; categorical was borderline significant) with alcohol was significantly associated with all cases after 20.3y f/u. However, aMED scores, with and without alcohol, were not significantly associated with total risk of advanced prostate cancer after 20.3y f/u. Modified Mediterranean Diet (mMED) score, with and without alcohol, was not significantly associated with risk of prostate cancer, including nonadvanced and advanced prostate cancer, after 20.3y f/u. Finally, WCRF/AICR diet only score, with and without alcohol, was not significantly associated with risk of prostate cancer, including nonadvanced and advanced prostate cancer, after 20.3y f/u.

Shin et $\mathrm{al}^{49}$ identified 3 dietary patterns using exploratory factor analysis in a cohort of men form Japan. Results showed that higher adherence to a "westernized pattern" at $56 y$ was associated with significantly increased risk of total and localized prostate cancer after 13.8y f/u, but was not significantly associated with risk of advanced prostate cancer. Additionally, adherence to the "prudent pattern" and the "traditional pattern" at 56y were not significantly associated with risk of advanced prostate cancer after 13.8y f/u.

Finally, Tantamango-Bartley et al ${ }^{50}$ examined various iterations of a vegetarian diet in relation to risk of prostate cancer. Results showed that consuming a vegan diet vs. a nonvegetarian diet was associated with a significantly lower risk of prostate cancer after 7.8y f/u. However, when stratified by race, results were only significant in white participants and not in black participants. In addition, consuming vegan, vegetarian, and nonvegetarian diets were not significantly associated with risk of advanced prostate cancer. 


\section{Assessment of the evidence ${ }^{i x}$}

This systematic review update included 8 studies that met inclusion criteria, and all were observational studies. The studies were heterogeneous, both in terms of which dietary patterns were examined, how dietary intake was assessed, in duration of f/u, and in direct and magnitude of effect of reported results. Based on this assessment, a conclusion was drawn indicating that limited evidence suggests no relationship between dietary patterns and risk of prostate cancer.

Publication bias is always a consideration, however it is not a serious concern for this body of evidence because a mix of significant and non-significant findings were reported.

As outlined and described below, the body of evidence examining dietary patterns and risk of prostate cancer was assessed for the following elements used when grading the strength of evidence.

- Risk of bias: The included studies had a number of potential risks of bias, or limitations that may have impacted study results (Table 12; Table 14). While studies adjusted for a number of potential confounders, they did not adjust for all key confounders, such as race/ethnicity. All studies examined dietary patterns once at baseline or in the first few years of $f / u$, and did not account for possible changes in dietary intake that may have occurred over $\mathrm{f} / \mathrm{u}$. In addition, the studies enrolled older individuals, and did not account for dietary patterns consumed earlier in life. None of the studies fully accounted for and/or analyzed the impact of missing data, either due to lost to $f / u$ or criteria used when selecting participants into the analyses. Finally, because preregistered statistical plans were not available for many of the included articles, the risk of potential selective outcome reporting is unclear.

- Consistency: The direction and magnitude of effect across the body of evidence was inconsistent. Most studies reported no significant associations between adherence to a dietary pattern and risk of prostate cancer. Though some studies reported significant associations depending on prostate cancer type (advanced, nonadvanced), whether dietary pattern score was examined categorically or continuously, if alcohol was included or excluded from the dietary pattern score, or subject characteristics (e.g., race), the reported associations were not consistent across the body of evidence.

- Directness: The populations, intervention, comparators, and outcomes of interest in the included studies are directly related to the systematic review question.

\footnotetext{
${ }^{i x} A$ detailed description of the methodology used for grading the strength of the evidence is available on the NESR website: https://nesr.usda.gov/2020-dietary-guidelines-advisory-committee-systematicreviews and in Part $\mathrm{C}$ of the following reference: Dietary Guidelines Advisory Committee. 2020. Scientific Report of the 2020 Dietary Guidelines Advisory Committee: Advisory Report to the Secretary of Agriculture and the Secretary of Health and Human Services. U.S. Department of Agriculture, Agricultural Research Service, Washington, DC.
} 
- Precision: Though the included studies did not report power analyses or sample size calculations, the majority had large analytic sample sizes with a sufficient number of prostate cancer cases occurring over f/u to examine associations. However, the width of confidence intervals indicates some degree of imprecision within the body of evidence.

- Generalizability: The study participants, interventions and/or exposures, comparators, and outcomes examined in the body of evidence are applicable to the U.S. population.

This systematic review updates the conclusion drawn by the 2015 Committee. ${ }^{x}$ The 2015 Committee concluded that, "No conclusion can be drawn regarding the relationship between dietary patterns and the risk of prostate cancer. This is due to limited evidence from a small number of studies with wide variation in study design, dietary assessment methodology and prostate cancer outcome ascertainment. (Grade: Grade Not Assignable)." This conclusion was based on seven prospective cohort studies published between January, 2000 and January, 2014. The 2015 Committee noted that most of the studies included in that review did not detect clear or consistent relationships between dietary patterns and risk of prostate cancer. The 2020 Dietary Guidelines Advisory Committee determined that, based on the 8 additional studies in this update, there is now limited evidence to suggest no relationship between dietary patterns and risk of prostate cancer.

\section{Research recommendations}

The 2020 Committee concurs with the 2015 Committee that in order to better assess the relationship between dietary patterns and risk of developing prostate cancer, additional research is needed to:

1. Investigate how dietary patterns consumed across the life cycle impact the risk of prostate cancer later in life, including childhood and adolescence, mid-life and later years.

2. Examine the impact of other potential confounders on the relationship between dietary patterns and prostate cancer risk, including PSA and DRE screening history, family history and genetics and the use of pharmaceutical agents impacting hormonal status.

3. Examine dietary patterns and associations with prostate cancer risk among diverse ethnic/racial minority groups in the United States.

4. Continue to explore the role of energy balance and obesity (including patterns of weight change throughout the life cycle), anthropometrics and physical activity in

\footnotetext{
x Dietary Guidelines Advisory Committee. Scientific Report of the 2015 Dietary Guidelines Advisory Committee: Advisory Report to the Secretary of Health and Human Services and the Secretary of Agriculture. US Department of Agriculture, Agricultural Research Service. https://health.gov/ourwork/food-nutrition/2015-2020-dietary-guidelines/advisory-report. Published 2015. Accessed April 30, 2020.
} 
prostate cancer risk.

5. Continue to define the role of specific nutrients, phytochemicals and foods that may individually or in combination during various stages of the life cycle impact the risk of prostate cancer.

6. When designing studies and conducting data analyses consider the possibility that men consuming a protective dietary pattern may also be living longer with less comorbidity, less competing mortality and may be more likely to be screened over a longer time interval (higher chance of detecting prostate cancers).

7. Recognize that prostate cancer is a heterogeneous collection of diseases and that future evaluation of dietary patterns, as well as specific nutrients and dietary components, may be more informative when considering specific subtypes defined by aggressiveness or molecular phenotyping. 


\section{Included articles}

1. Prentice RL, Aragaki AK, Howard BV, et al. Low-fat dietary pattern among postmenopausal women influences long-term cancer, cardiovascular disease, and diabetes outcomes. J Nutr. 2019;149(9):1565-1574. doi:10.1093/jn/nxz107.

2. Thomson CA, Van Horn L, Caan BJ, et al. Cancer incidence and mortality during the intervention and postintervention periods of the Women's Health Initiative dietary modification trial. Cancer Epidemiol Biomarkers Prev. 2014;23(12):2924-2935. doi:10.1158/1055-9965.Epi-14-0922.

3. Toledo E, Salas-Salvado J, Donat-Vargas C, et al. Mediterranean diet and invasive breast cancer risk among women at high cardiovascular risk in the PREDIMED Trial: a randomized clinical trial. JAMA Intern Med. 2015;175(11):1752-1760. doi:10.1001/jamainternmed.2015.4838.

4. Deschasaux M, Huybrechts I, Murphy N, et al. Nutritional quality of food as represented by the FSAm-NPS nutrient profiling system underlying the Nutri-Score label and cancer risk in Europe: results from the EPIC prospective cohort study. PLoS Med. 2018;15(9):e1002651. doi:10.1371/journal.pmed.1002651.

5. Deschasaux M, Julia C, Kesse-Guyot E, et al. Are self-reported unhealthy food choices associated with an increased risk of breast cancer? Prospective cohort study using the British Food Standards Agency nutrient profiling system. BMJ Open. 2017;7(6):e013718. doi:10.1136/bmjopen-2016-013718.

6. Donnenfeld M, Julia C, Kesse-Guyot E, et al. Prospective association between cancer risk and an individual dietary index based on the British Food Standards Agency Nutrient Profiling System. Br J Nutr. 2015;114(10):1702-1710. doi:10.1017/s0007114515003384.

7. Fiolet T, Srour B, Sellem L, et al. Consumption of ultra-processed foods and cancer risk: results from NutriNet-Sante prospective cohort. BMJ. 2018;360:k322.

doi:10.1136/bmj.k322.

8. Guinter MA, McLain AC, Merchant AT, Sandler DP, Steck SE. A dietary pattern based on estrogen metabolism is associated with breast cancer risk in a prospective cohort of postmenopausal women. Int J Cancer. 2018;143(3):580-590. doi:10.1002/ijc.31387.

9. Guinter MA, Sandler DP, McLain AC, Merchant AT, Steck SE. An estrogen-related dietary pattern and postmenopausal breast cancer risk in a cohort of women with a family history of breast cancer. Cancer Epidemiol Biomarkers Prev. 2018;27(10):12231226. doi:10.1158/1055-9965.Epi-18-0514.

10. Haridass V, Ziogas A, Neuhausen SL, Anton-Culver H, Odegaard AO. Diet quality scores inversely associated with postmenopausal breast cancer risk are not associated with premenopausal breast cancer risk in the California Teachers Study. $J$ Nutr. 2018;148(11):1830-1837. doi:10.1093/jn/nxy187.

11. Harris HR, Bergkvist L, Wolk A. An estrogen-associated dietary pattern and breast cancer risk in the Swedish Mammography Cohort. Int J Cancer. 2015;137(9):21492154. doi:10.1002/ijc.29586.

12. Harris HR, Willett WC, Vaidya RL, Michels KB. Adolescent dietary patterns and premenopausal breast cancer incidence. Carcinogenesis. 2016;37(4):376-384. doi:10.1093/carcin/bgw023. 
13. Harris HR, Willett WC, Vaidya RL, Michels KB. An adolescent and early adulthood dietary pattern associated with inflammation and the incidence of breast cancer. Cancer Res. 2017;77(5):1179-1187. doi:10.1158/0008-5472.Can-16-2273. 14. Kane-Diallo A, Srour B, Sellem L, et al. Association between a pro plant-based dietary score and cancer risk in the prospective NutriNet-sante cohort. Int $\mathrm{J}$ Cancer. 2018;143(9):2168-2176. doi:10.1002/ijc.31593.

15. Kojima R, Okada E, Ukawa S, et al. Dietary patterns and breast cancer risk in a prospective Japanese study. Breast Cancer. 2017;24(1):152-160. doi:10.1007/s12282016-0689-0.

16. Lavalette C, Adjibade M, Srour B, et al. Cancer-specific and general nutritional scores and cancer risk: results from the prospective NutriNet-Sante cohort. Cancer Res. 2018;78(15):4427-4435. doi:10.1158/0008-5472.Can-18-0155.

17. Li Y, Roswall N, Sandin S, Strom P, Adami HO, Weiderpass E. Adherence to a healthy Nordic food index and breast cancer risk: results from a Swedish cohort study. Cancer Causes Control. 2015;26(6):893-902. doi:10.1007/s10552-015-0564-X. 18. McKenzie F, Ferrari $\mathrm{P}$, Freisling $\mathrm{H}$, et al. Healthy lifestyle and risk of breast cancer among postmenopausal women in the European Prospective Investigation into Cancer and Nutrition cohort study. Int J Cancer. 2015;136(11):2640-2648.

doi:10.1002/ijc.29315.

19. Nomura SJ, Dash C, Rosenberg L, Yu J, Palmer JR, Adams-Campbell LL.

Adherence to diet, physical activity and body weight recommendations and breast cancer incidence in the Black Women's Health Study. Int J Cancer. 2016;139(12):27382752. doi:10.1002/ijc.30410.

20. Penniecook-Sawyers JA, Jaceldo-Siegl K, Fan J, et al. Vegetarian dietary patterns and the risk of breast cancer in a low-risk population. Br J Nutr. 2016;115(10):17901797. doi:10.1017/s0007114516000751.

21. Petimar J, Park YM, Smith-Warner SA, Fung TT, Sandler DP. Dietary index scores and invasive breast cancer risk among women with a family history of breast cancer. Am J Clin Nutr. 2019;109(5):1393-1401. doi:10.1093/ajcn/nqy392.

22. Shin S, Saito E, Inoue M, et al. Dietary pattern and breast cancer risk in Japanese women: the Japan Public Health Center-based Prospective Study (JPHC Study). Br J Nutr. 2016;115(10):1769-1779. doi:10.1017/s0007114516000684.

23. van den Brandt PA, Schulpen M. Mediterranean diet adherence and risk of postmenopausal breast cancer: results of a cohort study and meta-analysis. Int $\mathrm{J}$ Cancer. 2017;140(10):2220-2231. doi:10.1002/ijc.30654.

24. Voortman T, Kiefte-de Jong JC, Ikram MA, et al. Adherence to the 2015 Dutch dietary guidelines and risk of non-communicable diseases and mortality in the Rotterdam Study. Eur J Epidemiol. 2017;32(11):993-1005. doi:10.1007/s10654-0170295-2.

25. Catsburg C, Kim RS, Kirsh VA, Soskolne CL, Kreiger N, Rohan TE. Dietary patterns and breast cancer risk: a study in 2 cohorts. Am J Clin Nutr. 2015;101(4):817-823. doi:10.3945/ajcn.114.097659.

26. Pot GK, Stephen AM, Dahm CC, et al. Dietary patterns derived with multiple methods from food diaries and breast cancer risk in the UK Dietary Cohort Consortium. Eur J Clin Nutr. 2014;68(12):1353-1358. doi:10.1038/ejcn.2014.135. 
27. Boden S, Myte R, Wennberg M, et al. The inflammatory potential of diet in determining cancer risk; a prospective investigation of two dietary pattern scores. PLoS One. 2019;14(4):e0214551. doi:10.1371/journal.pone.0214551.

28. Fasanelli F, Zugna D, Giraudo MT, et al. Abdominal adiposity is not a mediator of the protective effect of Mediterranean diet on colorectal cancer. Int $J$ Cancer. 2017;140(10):2265-2271. doi:10.1002/ijc.30653.

29. Jones P, Cade JE, Evans CEL, Hancock N, Greenwood DC. The Mediterranean diet and risk of colorectal cancer in the UK Women's Cohort Study. Int J Epidemiol. 2017;46(6):1786-1796. doi:10.1093/ije/dyx155.

30. Kumagai Y, Chou WT, Tomata Y, et al. Dietary patterns and colorectal cancer risk in Japan: the Ohsaki Cohort Study. Cancer Causes Control. 2014;25(6):727-736. doi:10.1007/s10552-014-0375-5.

31. Liu L, Nishihara R, Qian ZR, et al. Association between inflammatory diet pattern and risk of colorectal carcinoma subtypes classified by immune responses to tumor. Gastroenterology. 2017;153(6):1517-1530.e1514. doi:10.1053/j.gastro.2017.08.045. 32. Mehta RS, Song M, Nishihara R, et al. Dietary patterns and risk of colorectal cancer: analysis by tumor location and molecular subtypes. Gastroenterology. 2017;152(8):1944-1953.e1941. doi:10.1053/j.gastro.2017.02.015.

33. Orlich MJ, Singh PN, Sabate J, et al. Vegetarian dietary patterns and the risk of colorectal cancers. JAMA Intern Med. 2015;175(5):767-776.

doi:10.1001/jamainternmed.2015.59.

34. Park SY, Boushey CJ, Wilkens LR, Haiman CA, Le Marchand L. High-quality diets associate with reduced risk of colorectal cancer: analyses of diet quality indexes in the Multiethnic Cohort. Gastroenterology. 2017;153(2):386-394.e382.

doi:10.1053/j.gastro.2017.04.004.

35. Petimar J, Smith-Warner SA, Fung TT, et al. Recommendation-based dietary indexes and risk of colorectal cancer in the Nurses' Health Study and Health Professionals Follow-up Study. Am J Clin Nutr. 2018;108(5):1092-1103. doi:10.1093/ajcn/nqy171.

36. Roswall N, Li Y, Kyro C, et al. No association between adherence to a healthy Nordic food index and colorectal cancer: results from a Swedish cohort study. Cancer Epidemiol Biomarkers Prev. 2015;24(4):755-757. doi:10.1158/1055-9965.Epi-14-1314. 37. Shin S, Saito E, Sawada N, et al. Dietary patterns and colorectal cancer risk in middle-aged adults: a large population-based prospective cohort study. Clin Nutr. 2018;37(3):1019-1026. doi:10.1016/j.clnu.2017.04.015.

38. Tabung FK, Liu L, Wang W, et al. Association of dietary inflammatory potential with colorectal cancer risk in men and women. JAMA Oncol. 2018;4(3):366-373.

doi:10.1001/jamaoncol.2017.4844.

39. Tabung FK, Wang W, Fung TT, et al. Association of dietary insulinemic potential and colorectal cancer risk in men and women. Am J Clin Nutr. 2018;108(2):363-370. doi:10.1093/ajcn/nqy093.

40. Torres Stone RA, Waring ME, Cutrona SL, Kiefe Cl, Allison J, Doubeni CA. The association of dietary quality with colorectal cancer among normal weight, overweight and obese men and women: a prospective longitudinal study in the USA. BMJ Open. 2017;7(6):e015619. doi:10.1136/bmjopen-2016-015619. 
41. Vargas AJ, Neuhouser ML, George SM, et al. Diet quality and colorectal cancer risk in the Women's Health Initiative Observational Study. Am J Epidemiol. 2016;184(1):2332. doi:10.1093/aje/kwv304.

42. Vulcan A, Ericson U, Manjer J, Ohlsson B. A colorectal cancer diet quality index is inversely associated with colorectal cancer in the Malmo diet and cancer study. Eur $J$ Cancer Prev. 2019;28(6):463-471. doi:10.1097/cej.0000000000000486.

43. Cheng E, Um CY, Prizment AE, Lazovich D, Bostick RM. Evolutionary-concordance lifestyle and diet and Mediterranean diet pattern scores and risk of incident colorectal cancer in lowa women. Cancer Epidemiol Biomarkers Prev. 2018;27(10):1195-1202. doi:10.1158/1055-9965.Epi-17-1184.

44. Schulpen M, van den Brandt PA. Mediterranean diet adherence and risk of colorectal cancer: the prospective Netherlands Cohort Study. Eur J Epidemiol. 2020;35(1):25-35. doi:10.1007/s10654-019-00549-8.

45. Anic GM, Park Y, Subar AF, Schap TE, Reedy J. Index-based dietary patterns and risk of lung cancer in the NIH-AARP diet and health study. Eur J Clin Nutr. 2016;70(1):123-129. doi:10.1038/ejcn.2015.122.

46. Hodge AM, Bassett JK, Shivappa N, et al. Dietary inflammatory index, Mediterranean diet score, and lung cancer: a prospective study. Cancer Causes Control. 2016;27(7):907-917. doi:10.1007/s10552-016-0770-1.

47. Maisonneuve P, Shivappa N, Hebert JR, et al. Dietary inflammatory index and risk of lung cancer and other respiratory conditions among heavy smokers in the COSMOS screening study. Eur J Nutr. 2016;55(3):1069-1079. doi:10.1007/s00394-015-0920-3. 48. Schulpen $M$, van den Brandt PA. Adherence to the Mediterranean diet and risk of lung cancer in the Netherlands Cohort Study. Br J Nutr. 2018;119(6):674-684. doi:10.1017/s0007114517003737.

49. Shin S, Saito E, Sawada N, et al. Dietary patterns and prostate cancer risk in Japanese: the Japan Public Health Center-based Prospective Study (JPHC Study). Cancer Causes Control. 2018;29(6):589-600. doi:10.1007/s10552-018-1030-3. 50. Tantamango-Bartley $\mathrm{Y}$, Knutsen SF, Knutsen R, et al. Are strict vegetarians protected against prostate cancer? Am J Clin Nutr. 2016;103(1):153-160. doi:10.3945/ajcn.114.106450. 51. Schulpen M, van den Brandt PA. Adherence to the Mediterranean diet and risks of prostate and bladder cancer in the Netherlands Cohort Study. Cancer Epidemiol Biomarkers Prev. 2019;28(9):1480-1488. doi:10.1158/1055-9965.Epi-19-0224. 
Table 1. Description of studies that examined the relationship between dietary patterns and breast cancer ${ }^{\mathbf{x}}$

\section{Study and \\ Participant \\ Characteristics \\ Randomized \\ Controlled Trials}

Intervention/Exposure and Outcomes

Results
Confounding, Study Limitations, and Summary of Findings

\section{Prentice, $2019^{1}$}

RCT (Women's Health Initiative Dietary Modification (DM) trial)

United States

Analytic N: 48835

(Intervention: 19541,

Comparison: 29294)

(Attrition: 0\%)

Participants were

$100 \%$ female, $\sim 62 \mathrm{y}$

$(50-79 \mathrm{y}), 28.2 \mathrm{~kg} / \mathrm{m} 2$,

$51 \%$ never smokers

\section{Dietary patterns:}

- Intervention group: Reduction in fat from $\sim 35 \% \mathrm{E}$ to $20 \% \mathrm{E}, 5$ servings/d fruits and vegetables, 6 serving/d grains; achieved reductions $8-10 \% \mathrm{E}$ of total, saturated, and unsaturated fat, reductions in $8-10 \%$ of carbohydrate, and increased vegetables, fruit, and grains vs. comparison.

- Comparison group: Received written healthrelated materials only; lower intakes of fruits, vegetables, whole grains, and total grains

Dietary assessment methods: Adherence was monitored using FFQs at baseline, 1y, and every $3 y$ thereafter

\section{Significant: N/A}

\section{Non-Significant:}

There were no significantly differences in postmenopausal invasive breast cancer risk during the intervention or after 19.6y $\mathrm{f} / \mathrm{u}$ between the intervention vs. comparison groups.

\section{Key confounders accounted for: N/A for RCTs \\ Other:}

Baseline hazard stratified on age at random assignment, ethnicity, hysterectomy status, prior disease (if applicable), randomization status in the hormone therapy trials, and study phase

\section{Limitations:}

- The intensity of the intervention may have differed between groups, as the intervention group received more intensive education than the comparison

Funding Sources: $\mathrm{NIH}$

Summary: There were no difference between intervention and comparison groups in risk of

\footnotetext{
${ }^{x i}$ Abbreviations: AHEI-2010, Alternative Healthy Eating Index-2010; aMED, Alternative Mediterranean Diet Score ; BMI, body mass index; CSDLH, Canadian Study of Diet, Lifestyle and Health; CVD, Cardiovascular disease; d, day; DASH, Dietary Approaches to Stop Hypertension; DM, dietary modification; DP, Dietary pattern; \%E, \% of energy; EPIC, European Prospective Investigation into Cancer and Nutrition; ERDP, Estrogen-related dietary pattern, EVOO, extra-virgin olive oil; FFQ, food frequency questionnaire; FSAm-NPS, Nutrient Profiling System of the British Food Standards Agency (modified version); f/u, follow-up; HNFI, Healthy Nordic food index; HR, hazard ratio; IMI, Italian Mediterranean Index; MEDI-LITE, Mediterranean diet score; MD, Mediterranean diet; MDS, Mediterranean Diet Score; mMED, modified Mediterranean diet score; mo, month(s); N/A, Not applicable; NBSS, National Breast Screening Study; NIH, National Institutes of Health; NOVA, Ultra-processed food score; NS, Not significant; NR, Not reported; PCS, prospective cohort study; pt, point; RCT, randomized controlled trial; SEER, Surveillance, Epidemiology, and End Results Program; WCRF/AICR, World Cancer Research Fund/American Institute for Cancer Research; wk, week(s); y, year(s)
} 


\section{Study and \\ Participant \\ Characteristics \\ Intervention/Exposure and Outcomes \\ Outcome assessment methods: US National \\ Cancer Institute's SEER system}

\section{Thomson, $2014^{2}$}

RCT (Women's Health Initiative Dietary Modification (DM) trial)

United States

Analytic N: 48835

(Intervention: 19541

Comparison: 29294)

(Attrition: 0\%)

Participants were

$100 \%$ female, $\sim 62 \mathrm{y}$

(50-79y), $28.2 \mathrm{~kg} / \mathrm{m} 2$,

$51 \%$ never smokers

\section{Dietary patterns:}

- Intervention group: Reduction in fat from $\sim 35 \%$ E to $20 \% E, 5$ servings/d fruits and vegetables, 6 serving/d grains; achieved reductions $8-10 \% \mathrm{E}$ of total, saturated, and unsaturated fat, reductions in $8-10 \%$ of carbohydrate, and increased vegetables, fruit, and grains vs. comparison.

- Comparison group: Received written healthrelated materials only; lower intakes of fruits, vegetables, whole grains, and total grains

Dietary assessment methods: Adherence was monitored using FFQs at baseline, 1y, and every $3 y$ thereafter

Outcome assessment methods: US National Cancer Institute's SEER system (Note: This paper also examined various molecular subtypes of breast cancer; results for molecular subtypes were not extracted in this table)
Results

Confounding, Study Limitations, and Summary of Findings

postmenopausal breast cancer during the

$8.5 y$ intervention or over $19.6 y \mathrm{f} / \mathrm{u}$.

\section{Significant:}

Women with higher baseline fat intake (quartiles) had significantly reduced risk of postmenopausal breast cancer $(p=0.03)$ :

- During the intervention: HR: 0.76, 95\% Cl: 0.62, 0.92

- During post-intervention f/u: HR: 1.11, 95\% Cl: $0.84,1.4$

\section{Non-Significant:}

There were no significant differences in invasive postmenopausal breast cancer risk after $5.2 \mathrm{y}, 8.5 \mathrm{y}$, or $13.5 \mathrm{y}$ f/u between the intervention vs. comparison groups.
Key confounders accounted for: N/A for RCTs

\section{Other: N/A}

\section{Limitations:}

- The intensity of the intervention may have differed between groups, as the intervention group received more intensive education than the comparison

\section{Funding Sources: NIH}

Summary: There were no difference between intervention and comparison groups in risk of invasive postmenopausal breast cancer during the $8.5 y$ intervention or over $19.6 \mathrm{y} / \mathrm{u}$.

However, women with higher baseline fat intake (quartiles) had significantly reduced risk of invasive postmenopausal breast cancer. 


\section{Study and}

Participant

Characteristics

Confounding, Study Limitations, and Summary of Findings

\section{Toledo, $2015^{3}$}

RCT (PREDIMED

Trial)

Spain

Analytic N: 4152

Participants were

$100 \%$ female, $60-80 y$

Excluded women with previous breast cancer ( $3 \%$ of original sample)

\section{Dietary patterns:}

- Mediterranean diet (MD) with extra-virgin olive oil (EVOO): MD supplemented with EVOO (provided 1L per week); MD diet included live oil for cooking and dressing; fruit, vegetables, legumes and fish; reduced total meat consumption, white meat instead of red or processed meat; homemade sauce with tomato, garlic, onion and spices with olive oil to dress vegetables, pasta, rice and other dishes; avoidance of butter, cream, fast food, sweets, pastries and sugar-sweetened beverages; and moderate red wine

- MD with mixed nuts: MD supplemented with mixed nuts (MD-nuts), with $30 \mathrm{~g}$ per day of mixed nuts $(15 \mathrm{~g}$ walnuts, $7.5 \mathrm{~g}$ hazelnuts and $7.5 \mathrm{~g}$ almonds)

- Control diet: Consumed similar food groups, but were counseled to also decrease fat intake in accordance with American Heart Association guidelines

Dietary assessment methods: Screening questionnaires were used to asses adherence to assigned diet

Outcome assessment methods: Medical records, death certificate review (Note: This paper also examined various molecular subtypes of breast cancer; results for molecular subtypes were not extracted in this table)

\section{Significant:}

Postmenopausal Breast cancer after 4.8y f/u:

- Control, $n=18 / 12$ 523: HR: 1.00

- MD+EVOO, n=17/5829: HR: 0.31, 95\% Cl: 0.13, 0.77

- MD+Nuts, $n=8 / 7031$ : HR: 0.53 , $95 \% \mathrm{Cl}: 0.23,1.26$

- $\quad$ MD diets combined, $n=10 / 5492$ : HR: $0.41,95 \% \mathrm{Cl}: 0.19,0.86$

Results were similar when excluding diagnosed within first year.

In the stratified analyses by age $(<$ or $>67 y$ ), smoking (never, ever), alcohol intake $(<$ or $>25 \mathrm{~g} / \mathrm{d})$, diabetes mellitus (yes, no), BMI (< or $>30 \mathrm{~kg} / \mathrm{m} 2)$, use of hormone therapy (yes, no), family history of cancer (no, yes), baseline MD adherence (low, high), all but 2 point estimates (for MD+nuts vs. control, among participants with $\mathrm{BMI}>30$ and for those with high baseline adherence to MD) showed an inverse association between the $M D+E V O O$ intervention and the incidence of breast cancer.

Non-Significant: N/A

\section{Key confounders accounted for:}

N/A for RCTs

\section{Other:}

Adjusted for Age, BMI, hormone therapy use, physical activity, total energy intake, alcohol consumption, age at menopause, baseline MD adherence, recruitment center, education to account for intra-cluster correlations in participants who were not individually randomized, considering as clusters the households and each of the clinics of Site D that were allocated in clusters

\section{Limitations:}

- Some concerns about bias due to randomization

Funding Sources: Spanish government (Instituto de Salud Carlos III); supplemental foods were donated by Patrimonio Comunal Olivarero and Hojiblanca (EVOO), California Walnut Commission (walnuts), Borges SA (almonds) and La Morella Nuts (hazelnuts)

Summary: Consuming a MD diet with EVOO or nuts was significantly decreased risk of postmenopausal breast cancer after $4.8 \mathrm{y} / \mathrm{fu}$, comapred to a control low-fat diet. 


\section{Study and}

Participant

Characteristics
Results
Confounding, Study Limitations, and Summary of Findings

Observational

Studies

\section{Catsburg, 2015 ${ }^{25}$}

Nested Case-Control Study and PCS (Canadian Study of Diet, Lifestyle and Health (CSDLH),

National Breast Screening Study (NBSS))

Canada

Analytic N: CSDLH: 4417, NBSS: 49410

Subjects were $100 \%$, female, $\sim 60$ yo (49-

$77 y)$ : CSDLH and 40$59 y:$ NBSS,

$\sim 23.5 \mathrm{~kg} / \mathrm{m}^{2}$ : CSDLH and NR: NBSS, $54 \%$ never smokers:

CSDLH and NR:

NBSS, $\sim 3.4 \mathrm{~g} / \mathrm{d}$

alchohol: CSDLH and

NR: NBSS

Exclusion criteria: NR

\section{Dietary patterns:}

Adherence to 3 dietary patterns identified using principal components factor analysis using CSDLH data, and replicated using confirmatory factor analysis using NBSS data:

- "Healthy" pattern: Higher loadings for vegetable and legume food groups

- "Ethnic" pattern: Higher loading for rice, spinach, fish, tofu, liver, eggs, and salted and dried meat

- "Meat and potatoes" pattern: Higher loadings for red meat groups and potatoes

Dietary assessment methods: 166 -item, validated FFQ at baseline (CSDLH); 86-item, validated FFQ at baseline (NBSS)

Outcome assessment methods: Canadian Cancer Registry, National Mortality Database

\section{Significant:}

"Healthy" pattern at $\sim 60 y$ (CSLDH) and total breast cancer after $13 \mathrm{y} / \mathrm{u}$ :

- Q1, n=125: HR: 1.00

- Q2, $\mathrm{n}=258: \mathrm{HR}: 0.93,95 \% \mathrm{Cl}$ : $0.74,1.18$

- Q3, $n=270: H R: 0.78,95 \% \mathrm{Cl}$ : $0.61,0.99$

- Q4, n=391: HR: $0.80,95 \% \mathrm{Cl}$ : $0.64,1.01$

- Q5, n=452: HR: $0.73,95 \% \mathrm{Cl}$ : $0.58,0.91$

- $\quad$-trend $=0.0001$

Results for the "Healthy" pattern were no longer significant when pre- and postmenopausal breast cancer were analyzed separately.

"Meat and potatoes" pattern at $\sim 60 \mathrm{y}$ (CSLDH) and postmenopausal breast cancer after $13 y \mathrm{f} / \mathrm{u}$ :

- Q1, n=57: HR: 1.00

- Q2, $\mathrm{n}=66$ : HR: $0.80,95 \% \mathrm{Cl}: 0.55$, 1.18

- Q3, n=148: HR: $1.49,95 \% \mathrm{Cl}$ : $1.07,2.07$

- Q4, n=149: HR: $1.21,95 \% \mathrm{Cl}$ : $0.87,1.69$

\section{Key confounders accounted for:}

Sex, age, physical activity, BMI, family history of the cancer outcome, aenopausal status

\section{Other:}

Energy intake, other dietary patterns

\section{Limitations:}

- Did not account for race/ethnicity, socioeconomic status, alcohol intake (in adults), smoking, hormonal contraceptive

- Criteria used to select subjects into the analysis not reported

- Only assessed dietary intake once at baseline; did not account for possible changes in dietary intake over $\mathrm{f} / \mathrm{u}$

- Did not account for missing data

- No preregistered statistical plan; potential for selective outcome reporting

Funding Sources: Breast Cancer Research Foundation

Summary: Higher adherence to the "Healthy" pattern in the CSLDH cohort at $60 y$ was associated with decreased risk of breast cancer after 13y f/u. However, results were not significant when pre- and postmenopausal women were analyzed separately. In addition, there was no significant associated between adherence to the "healthy" pattern at 40-59y 


\section{Study and}

Participant

Characteristics

\section{Results}

- Q5, n=205: HR: 1.26, 95\% Cl: $0.92,1.73$

- $\quad p$-trend $=0.043$

"Meat and potatoes pattern" at 40-59y (NBSS) and postmenopausal breast cancer after $\sim 23 \mathrm{y}$ f/u:

- $Q 1, n=358: H R: 1.00$

- Q2, n=361: HR: $1.03,95 \% \mathrm{Cl}$ : $0.85,1.24$

- Q3, n=399: HR: $1.22,95 \% \mathrm{Cl}$ : $0.98,1.52$

- Q4, n=365: HR: $1.17,95 \% \mathrm{Cl}$ : $0.91,1.50$

- Q5, n=338: HR: $1.31,95 \% \mathrm{Cl}$ : $0.98,1.76$

- $\quad p$-trend $=0.043$

\section{Non-Significant:}

"Healthy pattern" at 40-59y (NBSS) was not significantly associated with breast cancer after $\sim 23 \mathrm{f} / \mathrm{u}$, in all women combined and when pre- and postmenopausal women were analyzed separately.

"Meat and potatoes" pattern at 40-59y (NBSS) was not significantly associated with breast cancer after $\sim 23 \mathrm{f} / \mathrm{u}$, in all women combined or in premenopausal women.
Confounding, Study Limitations, and Summary of Findings

in the NBSS cohort and risk of breast cancer after $23 y \mathrm{f} / \mathrm{u}$.

Adherence to the "Ethnic" pattern was not significantly associated with risk of breast cancer in either the CSLDH or NBSS cohort.

Higher adherence to the "Meat and potatoes" pattern was associated with increased risk of postmenopausal breast cancer in both the CSLDH and NBSS cohorts. However, the "Meat and potatoes" pattern was not associated with total risk of breast cancer or risk of premenopausal breast cancer in either cohort. 
"Meat and potatoes" pattern at $\sim 60 \mathrm{y}$

(CSLDH) was not significantly associated with breast cancer after $13 \mathrm{y} / \mathrm{u}$, in all women combined or in premenopausal women.

"Ethnic" pattern at 40-59y (NBSS) was not significantly associated with breast cancer after $\sim 23 \mathrm{y} / \mathrm{u}$, in all women combined and when pre- and postmenopausal women were analyzed separately.

"Ethnic" pattern at 60y (CSLDH) was not significantly associated with breast cancer after 13y $f / u(p=0.073)$, in all women

combined and when pre- and

postmenopausal women were analyzed separately.

\section{Deschasaux, $2018^{4}$}

PCS (European

Prospective

Investigation into

Cancer and Nutrition

(EPIC))

Denmark, France,

Germany, Greece,

Italy, the Netherlands,

Norway, Spain,

Sweden, UK

Analytic N: 330766

\section{Dietary patterns:}

Nutrient Profiling System of the British Food Standards Agency (modified version) (FSAmNPS) score, categorical (quintiles) and continuous (per 2 pt increment)

- Overall diet score assigned based on energy, sugar, saturated fatty acid, sodium, fibers, proteins, and

fruits/vegetables/legumes/nuts.

- Higher FSAm-NPS score had higher intakes of alcohol, energy and red and processed meat, lower intakes of dietary fibers, vegetables, fruit, fish, and lean meat

\section{Significant:}

FSAm-NPS score at $51 \mathrm{y}$ and

postmenopausal breast cancer after 15.3y f/u:

- Q1, n=2093: HR: 1.00

- Q2, n=2303: HR: $1.04,95 \% \mathrm{Cl}$ $0.98,1.1$

- Q3, $n=2403: H R: 1.03,95 \% \mathrm{Cl}:$ $0.97,1.10$

- Q4, n=2682: HR: $1.07,95 \% \mathrm{Cl}$ : $1.01,1.14$

- Q5, $\mathrm{n}=2636: \mathrm{HR}: 1.06,95 \% \mathrm{Cl}$ : $0.99,1.14$

\section{Key confounders accounted for:}

Sex, age, education, alcohol intake, physical activity, smoking, BMI, height, family history of the cancer, hormonal contraceptive, menopausal status

\section{Other:}

Center, hormone replacement therapy, age at menarche, age at first full-term pregnancy, age at menopause, energy intake

\section{Limitations:}

- Did not account for race/ethnicity 


\section{Study and \\ Participant \\ Characteristics}

Intervention/Exposure and Outcomes

Results

Confounding, Study Limitations, and Summary of Findings

Dietary assessment methods: FFQs or 7-day

Subjects were $100 \%$ female, $\sim 51$ yo, $\sim 25.4$ $\mathrm{kg} / \mathrm{m}^{2}, 43 \%$ neversmokers, $\sim 5.3 \mathrm{~g} / \mathrm{d}$ alcohol

Excluded those with prevalent cancer; cancer diagnosis in first $2 y$ of $\mathrm{f} / \mathrm{u}$; missing data; implausible energy intake ( 10\% of original sample)

\section{Deschasaux, $2017^{5}$}

PCS (NutriNet-Santé)

France

Analytic N: 46864

Subjects were $100 \%$ female, $\sim 51$ yo, $\sim 24.1$ $\mathrm{kg} / \mathrm{m}^{2}, 48 \%$ neversmokers, $\sim 6.5 \mathrm{~g} / \mathrm{d}$ alcohol

Excluded those with prevalent cancer; $<35$ yo at baseline; at diet records, validated, at baseline, age $\sim 51 \mathrm{y}$

Outcome assessment methods: Record

linkage with population-based cancer registries, health insurance records, pathology registries, and f/u with study subjects

\section{Dietary patterns:}

Nutrient Profiling System of the British Food Standards Agency (modified version) (FSAmNPS) score, categorical (quintiles) and continuous (per 2 pt increment)

- Overall diet score assigned based on energy, sugar, saturated fatty acid, sodium, fibers, proteins, and

fruits/vegetables/legumes/nuts.

- Higher FSAm-NPS score had higher intakes of protein, fiber, fruit, vegetables, legumes, poultry, fish, and dairy, and lower intakes of energy, alcohol, fat, carbohydrate, red meat, processed meat,
- $\quad$-trend $=0.05$

Continuous, per $2 \mathrm{pt}$ increment, $\mathrm{n}=12063$ : HR: 1.02 , 95\% Cl: 1.00, 1.04; p-trend $=0.05$

\section{Non-Significant: N/A}

\section{Significant:}

FSAm-NPS score at 51y and total breast cancer after $4 \mathrm{y}$ f/u:

- Q1, $n=82: H R: 1.00$

- Q2, n= 122: HR: $1.43,95 \% \mathrm{Cl}$ : $1.08,1.90$

- Q3, $n=117: H R: 1.43,95 \% \mathrm{Cl}$ : 1.07, 1.91

- Q4, $n=138: H R: 1.79,95 \% \mathrm{Cl}$ : $1.35,2.38$

- $Q 5, n=96: H R: 1.52 v 1.11,2.08$

- $p$-trend $=0.002$

FSAm-NPS score, continuous, and total breast cancer, $n=555$ : HR: $1.06,95 \% \mathrm{Cl}$ : $1.02,1.11 \mathrm{p}$-trend $=0.005$
- Only assessed dietary intake once at baseline; did not account for possible changes in dietary intake over $\mathrm{f} / \mathrm{u}$

- No preregistered statistical plan; potential for selective outcome reporting

Funding Sources: French National Cancer Institute, European Commission, the International Agency for Research on Cancer

Summary: Consuming a diet that scores higher on the Nutrient Profiling System of the British Food Standards Agency (modified version) (FSAm-NPS) at 51y was associated with increased risk of postmenopausal breast cancer after 15.3y f/u.

\section{Key confounders accounted for:}

Sex, Age, race/ethnicity, education, alcohol intake, physical activity, smoking, BMI, height, family history of cancer, hormonal contraceptive, menopausal status

\section{Other:}

Number of dietary records, energy intake, number of biological children, hormonal treatment for menopause

\section{Limitations:}

- Did not account for race/ethnicity

- Only assessed dietary intake during first $2 y$ of $f / u$; did not account for possible changes in dietary intake over f/u 


\begin{tabular}{|c|c|c|c|}
\hline $\begin{array}{l}\text { Study and } \\
\text { Participant } \\
\text { Characteristics }\end{array}$ & Intervention/Exposure and Outcomes & Results & $\begin{array}{l}\text { Confounding, Study Limitations, and } \\
\text { Summary of Findings }\end{array}$ \\
\hline $\begin{array}{l}\text { least } 3 \text { valid } 24-\mathrm{hr} \\
\text { dietary records during } \\
\text { first } 2 \mathrm{y} \text { f/u }(\sim 40 \% \text { of } \\
\text { original sample) }\end{array}$ & $\begin{array}{l}\text { Dietary assessment methods: } 3 \text {, 24-hour } \\
\text { dietary recalls, assessed every } 6 \text { mo during the } \\
\text { first } 2 y \text { of f/u, at age } \sim 49 y \\
\text { Outcome assessment methods: } \\
\text { Participant report, medical record review, } \\
\text { pathological reports }\end{array}$ & 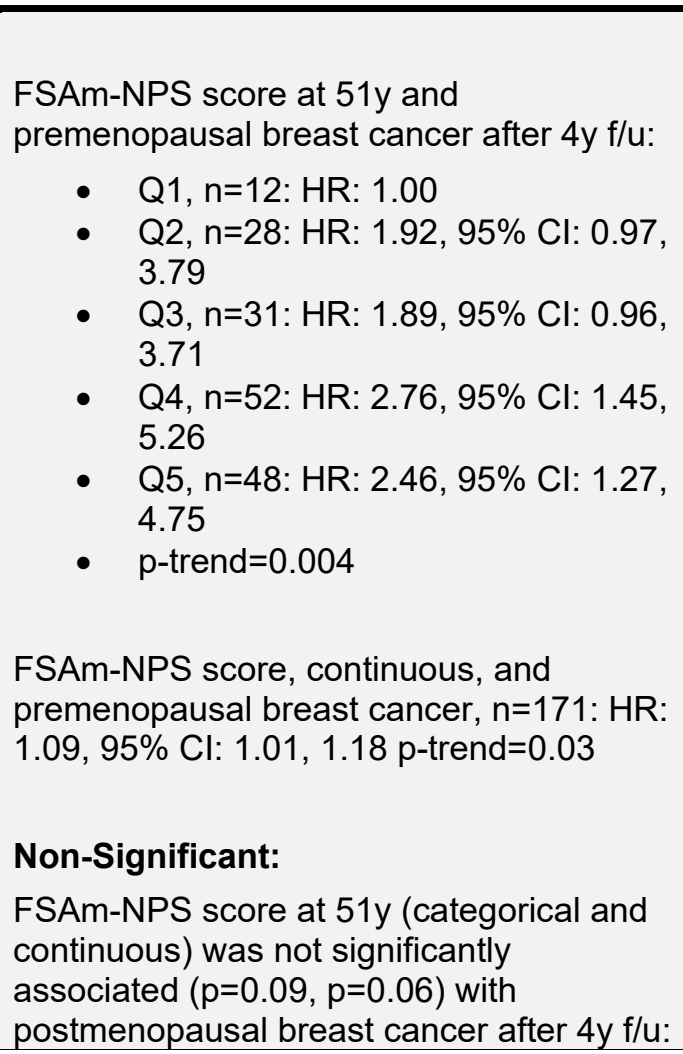 & $\begin{array}{l}\text { - No preregistered statistical plan; potential } \\
\text { for selective outcome reporting } \\
\text { Funding Sources: Région lle-de-France, } \\
\text { Cancéropôle lle-de-France and CORDDIM), } \\
\text { Ministère de la Santé, Institut de Veille } \\
\text { Sanitaire (InVS), Institut National de la } \\
\text { Prévention etde l'Education pour la Santé } \\
\text { (INPES), Région Ile-de-France (CORDDIM), } \\
\text { Institut Nationalde la Santé et de la } \\
\text { Recherche Médicale (INSERM), Institut } \\
\text { National de la Recherche Agronomique } \\
\text { (INRA), onservatoire National des Arts et } \\
\text { Métiers (CNAM) and Université Paris } 13 \\
\\
\text { Summary: Higher FSAm-NPS score at } 51 y \\
\text { was significantly associated with increased } \\
\text { risk of total and premenopausal breast cancer } \\
\text { after 4y f/u. However, FSAm-NPS score was } \\
\text { not significantly associated with risk of } \\
\text { postmenopausal breast cancer. }\end{array}$ \\
\hline $\begin{array}{l}\text { Donnenfeld, } 2015^{6} \\
\text { PCS } \\
\text { (SUpplémentation en } \\
\text { VItamines et Minéraux } \\
\text { AntioXydants cohort) }\end{array}$ & $\begin{array}{l}\text { Dietary patterns: } \\
\text { Nutrient Profiling System of the British Food } \\
\text { Standards Agency (modified version) (FSAm- } \\
\text { NPS) score, categorical (quintiles) and } \\
\text { continuous (per } 2 \text { pt increment) } \\
\text { - Overall diet score assigned based on } \\
\quad \text { energy, sugar, saturated fatty acid, sodium, }\end{array}$ & $\begin{array}{l}\text { Non-Significant: } \\
\text { FSAm-NPS score at } 49 y \text { (categorical and } \\
\text { continuous) was not significantly } \\
\text { associated with breast cancer after } 12.6 y \\
\text { f/u. }\end{array}$ & $\begin{array}{l}\text { Key confounders accounted for: } \\
\text { Sex, age, education, alcohol intake, physical } \\
\text { activity, smoking, BMI, height, family history of } \\
\text { the cancer, menopausal status } \\
\text { Other: } \\
\text { Intervention group of the initial SU.VI.MAX } \\
\text { trial, number of dietary records, hormone } \\
\text { replacement therapy, number of live births }\end{array}$ \\
\hline
\end{tabular}




\section{Study and \\ Participant \\ Characteristics}

Intervention/Exposure and Outcomes

Results

fibers, proteins, and

fruits/vegetables/legumes/nuts.

Analytic N: 6435

Subjects were $100 \%$ female, $49 y$ y, $34 \%$ $\mathrm{BMI}>25 \mathrm{~kg} / \mathrm{m}^{2}, 48 \%$ never-smokers,

$\sim 18.8 \mathrm{~g} / \mathrm{d}$ alcohol

Excluded those with prevalent cancer; cancer diagnosis in first 3y of f/u; $<6$ 24-hr recalls within the first $2 y$ of $f / u$; implausible energy intake $(\sim 51 \%$ of original sample)

\section{Fiolet, 2018}

PCS (NutriNet-Santé)

France

Analytic N: 104,980

Subjects were $100 \%$ female, 42.8 yo, $23.8 \mathrm{~kg} / \mathrm{m} 2 \mathrm{BMl}, 83 \%$ never or former smokers, $\sim 7.8 \mathrm{~g} / \mathrm{d}$ achohol
Dietary assessment methods: 3,24 -hour dietary recalls, assessed every 6 mo for the first $2 y$ of $f / u$, age $\sim 49 y$

\section{Outcome assessment methods:}

Participant report, medical record review, pathological reports

\section{Dietary patterns:}

- Ultra-processed food score (NOVA),

- Main food groups contributing to NOVA score were sugary drinks, drinks, starchy foods and breakfast cereals, ultra-processed fruits and vegetables, dairy products, meats, fish, and eggs, processed meats, fats, and salty snacks

Dietary assessment methods: 3, 24-hour dietary recalls, assessed every $6 \mathrm{mo}$ for the first $2 y$ of $f / u$, age $\sim 49 y$ categorical (quartiles)
Confounding, Study Limitations, and Summary of Findings

\section{Limitations:}

- Did not account for race/ethnicity, hormonal contraceptive use

- Only assessed dietary intake during first $2 y$ of $f / u$; did not account for possible changes in dietary intake over f/u

- No preregistered statistical plan; potential for selective outcome reporting

Funding Sources: French Ministry of Health (DGS), National Institute for Prevention and Health Education (INPES)

Summary: Nutrient Profiling System of the British Food Standards Agency (modified version) (FSAm-NPS) score at $49 y$ was not significantly associated with risk of breast cancer after 12.6y f/u.

\section{Significant:}

Ultra-processed food score, continuous, and total breast cancer $(n=739)$ : HR: 1.11 $95 \% \mathrm{Cl}: 1.01,1.21 ; \mathrm{p}$-trend $=0.03$

Ultra-processed food score at $49 y$ and postmenopausal breast cancer after $5.4 \mathrm{y}$ f/u:

\section{Q1, $n=90: H R: 1.00$}

Q2, n=70: HR: $1.23,95 \% \mathrm{Cl}: 0.95,1.59$

Q3, n=55: HR: $1.27,95 \% \mathrm{Cl}: 0.97,1.65$

Q4, n=49: HR: $1.38,95 \% \mathrm{Cl}: 1.05,1.81$

\section{Key confounders accounted for:}

Sex, age, education, alcohol intake, physical activity, smoking, BMI, height, family history of the cancer, hormonal contraceptive, menopausal status

\section{Other:}

Energy intake without alcohol, number of 24 hour dietary records, hormone replacement therapy, number of children, intakes of lipids, sodium, and carbohydrates, western dietary pattern

\section{Limitations:}

- Did not account for race/ethnicity 


\section{Study and}

Participant

Characteristics

Intervention/Exposure and Outcomes

Results

Outcome assessment methods:

p-trend $=0.02$

Excluded those with prevalent cancer; $<35 y$ at baseline; at least 2 valid $24-\mathrm{hr}$ dietary records during first $2 y$ f/u; diagnosis in first $2 y$ of $f / u(\sim 40 \%$ of original sample)
Participant report, medical record review, pathological reports
Ultra-processed food score, continuous, and postmenopausal breast cancer $(n=264):$ HR: $1.13,95 \%$ Cl: 1.00, 1.27; $p$ trend $=0.05$

\section{Non-Significant:}

Ultra-processed food score at $49 y$ (categorical) was not significantly associated with total breast cancer after $5.4 \mathrm{y} f / \mathrm{u}$.

Ultra-processed food score at $49 y$ (categorical and continuous) was not significantly associatef with premenopausal breast cancer after $5.4 \mathrm{y}$ f/u.
Confounding, Study Limitations, and Summary of Findings

- Only assessed dietary intake during first $2 y$ of f/u; did not account for possible changes in dietary intake over $\mathrm{f} / \mathrm{u}$

- No preregistered statistical plan; potential for selective outcome reporting

Funding Sources: Région lle-de-France, Cancéropôle lle-de-France and CORDDIM), Ministère de la Santé, Institut de Veille Sanitaire (InVS), Institut National de la Prévention etde l'Education pour la Santé (INPES), Région lle-de-France (CORDDIM), Institut Nationalde la Santé et de la Recherche Médicale (INSERM), Institut National de la Recherche Agronomique (INRA), onservatoire National des Arts et Métiers (CNAM) and Université Paris 13

Summary: Higher ultra-processed food score at $49 y$ was significantly associated with increased risk of postmenopausal breast cancer after $5.4 y$ f/u. It was also associated with higher risk of total breast cancer, when analyzed continuously.

However, ultra-processed food score at 49y was not significantly associated with risk of total breast cancer after $5.4 \mathrm{y} f / \mathrm{u}$, when analyzed categorically. It was also not associated with risk of premenopausal breast cancer (categorically or continuously).
Guinter, 2018a (IJC) 8
Dietary patterns:
PCS (Prostate, Lung,
Adherence to a dietary pattern identified using
Colorectal and

Significant:

\section{Key confounders accounted for:}

Sex, age, race/ethnicity, education, alcohol intake, physical activity,BMI, BMI at age 20y, 


\begin{tabular}{|c|c|c|c|}
\hline $\begin{array}{l}\text { Study and } \\
\text { Participant } \\
\text { Characteristics }\end{array}$ & Intervention/Exposure and Outcomes & Results & $\begin{array}{l}\text { Confounding, Study Limitations, and } \\
\text { Summary of Findings }\end{array}$ \\
\hline \multirow[t]{2}{*}{$\begin{array}{l}\text { Excluded men, } \\
\text { subjects without } \\
\text { complete data, } \\
\text { prevalent cancer } \\
\text { ( } 30 \% \text { of original } \\
\text { sample) }\end{array}$} & $\begin{array}{l}\text { reduced rank regression (response variables: } \\
\text { unconjugated estradiol, ratio of 2- and 16- } \\
\text { hydroxylated estrogen metabolites), categorical } \\
\text { (quartiles) and continuous: } \\
\text { - "Estrogen-related dietary pattern (ERDP)": } \\
\text { Higher loadings for non-whole/refined } \\
\text { grains, tomatoes, cruciferous vegetables, } \\
\text { cheese, high omega-3 fish/shellfish, } \\
\text { franks/luncheon meats, and lower in } \\
\text { nuts/seeds, other vegetables, low omega-3 } \\
\text { fish/shellfish, yogurt, coffee } \\
\text { Dietary assessment methods: } 137 \text {-item, } \\
\text { validated, FFQ t baseline, age } ~ 62 y \\
\text { Outcome assessment methods: Participant } \\
\text { f/u, National Death Index, physician reports, } \\
\text { state cancer registries, next of kin reports (Note: } \\
\text { This paper also examined various molecular } \\
\text { subtypes of breast cancer; results for molecular } \\
\text { subtypes were not extracted in this table) }\end{array}$ & 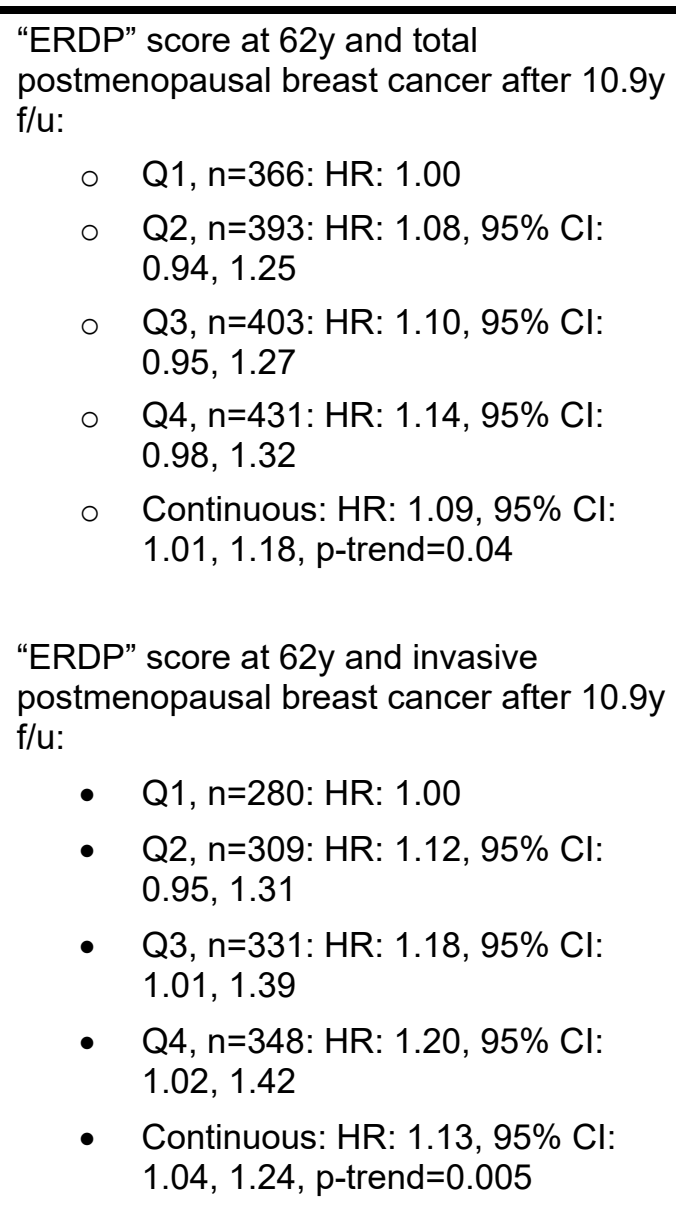 & $\begin{array}{l}\text { family history of the cancer outcome, } \\
\text { menopausal status } \\
\text { Other: } \\
\text { Hormone therapy, total energy intake, bilateral } \\
\text { oophorectomy, parity, recruitment center } \\
\text { Limitations: } \\
\text { - Did not account for smoking, hormonal } \\
\text { contraceptive use } \\
\text { - Only assessed dietary intake once at } \\
\text { baseline; did not account for possible } \\
\text { changes in dietary intake over f/u } \\
\text { - Did not account for missing data } \\
\text { - No preregistered statistical plan; potential } \\
\text { for selective outcome reporting } \\
\text { Funding Sources: NIH } \\
\text { Summary: Higher "ERDP" score at } 62 y \text { was } \\
\text { significantly associated with increased risk of } \\
\text { total and invasive breast cancer after } 10.9 y \\
\text { f/u. }\end{array}$ \\
\hline & & Non-Significant: N/A & \\
\hline $\begin{array}{l}\text { Guinter, 2018b } \\
\text { (CEBP) }\end{array}$ & Dietary patterns: & Significant: N/A & Key confounders accounted for: \\
\hline
\end{tabular}




\section{Study and \\ Participant}

Characteristics

Intervention/Exposure and Outcomes

Adherence to a dietary pattern identified using reduced rank regression (response variables: unconjugated estradiol, ratio of 2- and 16-

hydroxylated estrogen metabolites), categorical by quartile:

- "Estrogen-related dietary pattern" (ERDP): Higher loading for non-whole/refined grains, tomatoes, cruciferous vegetables, cheese, high omega-3 fish/shellfish, franks/luncheon meats, and lower in nuts/seeds, other vegetables, low omega-3 fish/shellfish, yogurt, coffee

Excluded subjects with prevalent cancer, premenopausal, extreme BMI, implausible energy intake, missing covariate data $(\sim 25 \%$ of original sample)
Dietary assessment methods: 110 -item, validated FFQ at baseline, $\sim 58 \mathrm{y}$

Outcome assessment methods: Self-report, confirmed by medical records (Note: This paper also examined various molecular subtypes of breast cancer; results for molecular subtypes were not extracted in this table)

\section{Results}

\section{Non-Significant:}

"ERDP" score at 58y was not significantly associated with total postmenopausal breast cancer after 6-12y f/u.

"ERDP" score at 58y was not significantly associated with invasive breast cancer after 6-12y f/u.
Confounding, Study Limitations, and Summary of Findings

Sex, Age, Race/ethnicity, Alcohol intake, BMI, $\mathrm{BMI}$ at $30 \mathrm{y}$, Family history of the cancer outcome, Menopausal status

\section{Other:}

Total energy intake, hormone therapy, age at menarche, parity, hysterectomy

\section{Limitations:}

- Did not account for socioeconomic status, physical activity, smoking, hormonal contraceptive use

- Only assessed dietary intake once at baseline; did not account for possible changes in dietary intake over $\mathrm{f} / \mathrm{u}$

- Did not account for missing data

- No preregistered statistical plan; potential for selective outcome reporting

Funding Sources: NIH, Susan G. Komen

Summary: "ERDP" score at 58y was not significantly associated with risk of total postmenopausal or invasive breast cancer after 6-12y f/u.

\section{Key confounders accounted for:}

Sex, age, race/ethnicity, socioeconomic status, alcohol intake, physical activity, smoking, BMI, family history of the cancer

PCS (California

Teachers Study)

- Alternative Mediterranean Diet Score $(\mathrm{aMED})^{\mathrm{xii}}$, with and without alcohol, categorical (quintiles)

\section{Non-Significant}

xii Fung TT, Hu FB, McCullough ML, Newby PK, Willett WC, Holmes MD. Diet quality is associated with the risk of estrogen receptor-negative breast cancer in postmenopausal women. J Nutr 2006;136:466-72. 


\begin{tabular}{|c|c|c|c|c|}
\hline $\begin{array}{l}\text { Study and } \\
\text { Participant } \\
\text { Characteristics }\end{array}$ & \multicolumn{2}{|c|}{ Intervention/Exposure and Outcomes } & Results & $\begin{array}{l}\text { Confounding, Study Limitations, and } \\
\text { Summary of Findings }\end{array}$ \\
\hline $\begin{array}{l}\text { United States } \\
\text { Analytic N: } 96959 \\
\text { Subjects were } 100 \% \\
\text { female, } 440 y 0 \text { (22- } \\
104 \mathrm{y}), \sim 24.4 \mathrm{~kg} / \mathrm{m} 2 \text {, } \\
\sim 24 \% \text { ever smoked, } \\
\sim 7 \mathrm{~g} / \mathrm{d} \text { alcohol } \\
\text { Excluded subjects with } \\
\text { self-reported history of } \\
\text { diabetes, heart attack, } \\
\text { stroke, or cancer; } \\
\text { excessive missing } \\
\text { data; implausible } \\
\text { energy intake }(\sim 73 \% \\
\text { of the original sample) }\end{array}$ & $\begin{array}{c}0 \\
0 \\
0 \\
\text { - DAS } \\
0 \\
\text { - AHE } \\
\text { cate } \\
0 \\
\\
\text { O } \\
\text { O } \\
\text { Pale }\end{array}$ & $\begin{array}{l}\text { Positive components: Vegetables (not } \\
\text { potatoes), legumes, fruits, nuts, whole } \\
\text { grains, fish, MUFA/SFAs } \\
\text { Neutral components: Alcohol } \\
\text { Negative components: Red and } \\
\text { processed meat } \\
\text { H Index xiii, categorical (quintiles) } \\
\text { Positive components: Vegetables (not } \\
\text { potatoes), nuts and legumes, fruit and } \\
\text { fruit juice, whole grains, low-fat dairy } \\
\text { Negative components: Red and } \\
\text { processed meat, sweetened } \\
\text { beverages, sodium } \\
\text { I-2010xiv, with and without alcohol, } \\
\text { gorical (quintiles) } \\
\text { Positive components: Vegetables (not } \\
\text { potatoes, French fries), Fruit, Whole } \\
\text { Grains, Long-Chain Fats (EPA + DHA), } \\
\text { PUFA } \\
\text { Neutral components: Alcohol } \\
\text { Negative components: Red and } \\
\text { Processed Meat, Sugar Sweetened } \\
\text { Beverages and Fruit Juice, Trans FA, } \\
\text { Sodium } \\
\text { o Index }\end{array}$ & $\begin{array}{l}\text { aMED score, with or without alcohol, at } \\
40 y \text { was not significantly associated with } \\
\text { postmenopausal breast cancer after } \sim 14 y \\
\text { f/u: } \\
\text { DASH score at } 40 y \text { was not significantly } \\
\text { associated with postmenopausal breast } \\
\text { cancer after } \sim 14 y \text { f/u. } \\
\text { AHEI-2010 score, with and without alcohol, } \\
\text { at } 40 y \text { was not significantly associated with } \\
\text { postmenopausal breast cancer after } \sim 14 y \\
\text { f/u. } \\
\text { Paleo score at } 40 y \text { was not significantly } \\
\text { associated with postmenopausal breast } \\
\text { cancer after } \sim 14 y \text { f/u. }\end{array}$ & $\begin{array}{l}\text { Outcome, hormonal contraceptive, } \\
\text { menopausal status } \\
\text { Other: } \\
\text { Age at menarche, parity, total energy intake } \\
\text { Limitations: } \\
\text { - Excluded subjects with chronic diseases } \\
\text { at baseline } \\
\text { - Only assessed dietary intake once at } \\
\quad \text { baseline; did not account for possible } \\
\quad \text { changes in dietary intake over f/u } \\
\text { - Did not account for missing data } \\
\text { - No preregistered statistical plan; potential } \\
\quad \text { for selective outcome reporting } \\
\text { Funding Sources: NIH, California Breast } \\
\text { Cancer Research Fund } \\
\text { Summary: aMED, DASH, AHEI-2010, and } \\
\text { Paleo scores at } 40 y \text { were not significantly } \\
\text { associated with risk of postmenopausal breast } \\
\text { cancer risk after 14y f/u. }\end{array}$ \\
\hline
\end{tabular}

xiii Fung TT, Hu FB, Hankinson SE, Willett WC, Holmes MD. Low carbohydrate diets, dietary approaches to stop hypertension-style diets, and the risk of postmenopausal breast cancer. Am J Epidemiol 2011;174:652-60.

xiv Chiuve SE, Fung TT, Rimm EB, Hu FB, McCullough ML, Wang M, Stampfer MJ, Willett WC. Alternative dietary indices both strongly predict risk of chronic disease. J Nutr 2012;142:1009-18.

xv Whalen KA, McCullough ML, Flanders WD, Hartman TJ, Judd S, Bostick RM. Paleolithic and Mediterranean diet pattern scores are inversely associated with biomarkers of inflammation and oxidative balance in adults. J Nutr 2016;146:1217-26. 
- Positive components: Vegetables, fruit and vegetable diversity, fruit, nuts, fish, lean meat, calcium (from Non-dairy foods)

- Negative components: Grains and starches, baked goods, red and processed meat, dairy foods, sugarsweetened beverages, alcohol, sodium

Dietary assessment methods: 103-item,

validated FFQ at baseline, $\sim 40 y$

Outcome assessment methods: California Cancer Registry

Harris, 2015
11
PCS (Swedish
Mammography
Cohort)
Sweden
Analytic N: 37004
Subjects were 100\%
female, $61.8 \mathrm{yo}$,
$\sim 25 \mathrm{~kg} / \mathrm{m} 2, \sim 22 \%$
current smokers,
$\sim 5 \mathrm{~g} / \mathrm{d}$ alcohol

\section{Dietary patterns:}

Adherence to a dietary pattern identified via reduced rank regression (response variables: estradiol, estrone sulfate), categorical (quartiles) and continuous:

- "Estrogen" dietary pattern: Higher loadings or red meat, legumes and pizza, and lower in coffee, whole grains

Dietary assessment methods: 96-item, validated FFQ at baseline, $\sim 62 \mathrm{y}$

\section{Outcome assessment methods: Swedish}

Cancer registers (Note: This paper also examined various molecular subtypes of breast

\section{Significant:}

"Estrogen" dietary pattern at $62 \mathrm{y}$ and postmenopausal breast cancer after $15 \mathrm{y}$ f/u:

- Q1, n=363: HR: 1.00

- Q2, n=401: HR: 1.10, 95\% Cl: $0.92,1.31$

- Q3, n=414: HR: $1.13,95 \% \mathrm{Cl}$ : $0.95,1.35$

- $Q 4, n=425: H R: 1.29,95 \% \mathrm{Cl}$ : $1.08,1.55$

- $p$-trend $=0.006$

Continuous: HR: $1.03,95 \% \mathrm{Cl}: 1.00,1.05$

\section{Key confounders accounted for:}

Sex, age, education, alcohol intake, physical activity, smoking, BMI, height, family history of the cancer outcome, hormonal contraceptive, menopausal status

\section{Other:}

Energy intake, hormone replacement therapy, age at menarche, history of benign breast disease

\section{Limitations:}

- Did not account for race/ethnicity

- Only assessed dietary intake once at baseline; did not account for possible changes in dietary intake over $\mathrm{f} / \mathrm{u}$ 


\begin{tabular}{|c|c|c|c|}
\hline $\begin{array}{l}\text { Study and } \\
\text { Participant } \\
\text { Characteristics }\end{array}$ & Intervention/Exposure and Outcomes & Results & $\begin{array}{l}\text { Confounding, Study Limitations, and } \\
\text { Summary of Findings }\end{array}$ \\
\hline $\begin{array}{l}\text { Excluded subjects with } \\
\text { prevalent cancer, } \\
\text { implausible energy } \\
\text { intake, or who had } \\
\text { completed the } 1997 \\
\text { questionnaire with } \\
\text { covariate data }(\sim 44 \% \\
\text { of original sample) }\end{array}$ & $\begin{array}{l}\text { cancer; results for molecular subtypes were not } \\
\text { extracted in this table) }\end{array}$ & Non-Significant: N/A & $\begin{array}{l}\text { - Did not account for missing data } \\
\text { - No preregistered statistical plan; potential } \\
\text { for selective outcome reporting } \\
\text { Funding Sources: Swedish Cancer } \\
\text { Foundation, Swedish Research } \\
\text { Council/Committee for Infrastructure, Swedish } \\
\text { Foundation for International Cooperation in } \\
\text { Research and Higher Education and the } \\
\text { Regional Research Fund Uppsala- } € \text { Orebro } \\
\text { Region } \\
\text { Summary: Higher adherence to an "estrogen" } \\
\text { dietary pattern at } 62 y \text { was significantly } \\
\text { associated with increased risk of breast } \\
\text { cancer after } 15 y \text { f/u. }\end{array}$ \\
\hline $\begin{array}{l}\text { Harris, } 2016 \\
12 \\
\text { PCS (Nurses' Health } \\
\text { Study II) } \\
\text { United States } \\
\text { Analytic N: } 45204 \\
\text { Subjects were } 100 \% \\
\text { female, } 41 \text { yo in } 1997 \text {, } \\
\sim 21 \mathrm{~kg} / \mathrm{m} 2 \text { at } 18 \mathrm{y} \\
\text { Excluded subjects } \\
\text { who did not complete } \\
\text { the high school FFQ, } \\
\text { implausible energy } \\
\text { intake, missing data, }\end{array}$ & $\begin{array}{l}\text { Dietary patterns: } \\
\text { Adherence to } 3 \text { dietary patterns identified using } \\
\text { principal components analysis, categorical } \\
\text { (quintiles): } \\
\text { - "Prudent" pattern: Higher loadings for } \\
\text { vegetables, fruits, legumes, fish and } \\
\text { poultry } \\
\text {-Western" pattern: Higher loadings for } \\
\text { refined grains, red and processed } \\
\text { meats, sweets and potatoes } \\
\text { - "Fast-food" pattern: Higher loadings for } \\
\text { pizza, fries, sweets and soda }\end{array}$ & 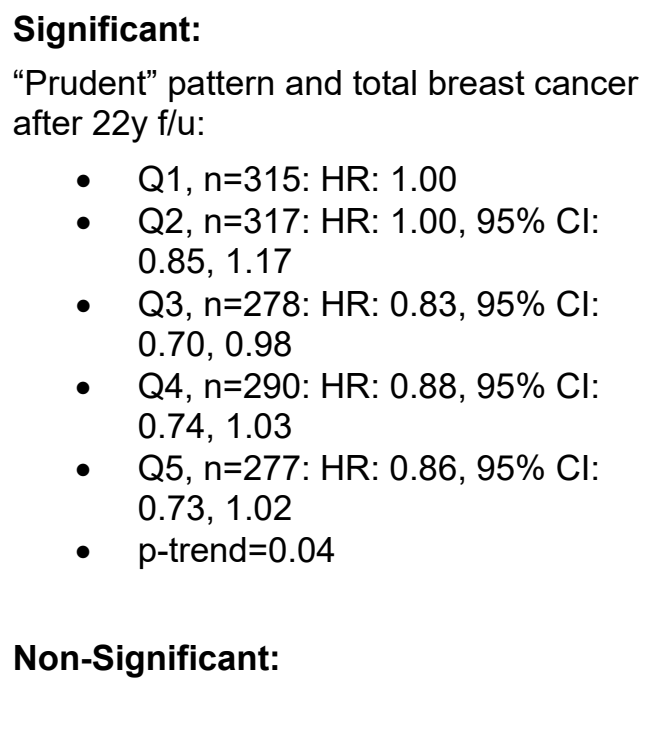 & $\begin{array}{l}\text { Key confounders accounted for: } \\
\text { Sex, age, alcohol intake (in adults), physical } \\
\text { activity, height at } 18 \mathrm{y}, \text { BMl at 18y, weight } \\
\text { change since } 18 y, \text { family history of the cancer } \\
\text { outcome, hormonal contraceptive, } \\
\text { menopausal status } \\
\text { Other: Energy intake in high school, age at } \\
\text { menarche, age at first birth/parity, history of } \\
\text { benign breast disease, hormone use } \\
\text { Limitations: } \\
\text { - Did not account for race/ethnicity, } \\
\text { socioeconomic status, smoking } \\
\text { - Only assessed dietary intake once at } \\
\text { baseline; did not account for possible } \\
\text { changes in dietary intake over f/u }\end{array}$ \\
\hline
\end{tabular}




\begin{tabular}{|c|c|c|c|}
\hline $\begin{array}{l}\text { Study and } \\
\text { Participant } \\
\text { Characteristics }\end{array}$ & Intervention/Exposure and Outcomes & Results & $\begin{array}{l}\text { Confounding, Study Limitations, and } \\
\text { Summary of Findings }\end{array}$ \\
\hline $\begin{array}{l}\text { prevalent cancer } \\
\text { before } 1989(\sim 61 \% \text { of } \\
\text { the original sample) }\end{array}$ & $\begin{array}{l}\text { Alternative Healthy Eating Index } \\
\text { (quintiles) } \\
\text { - } \quad \text { Positive components: Vegetables (not } \\
\text { potatoes, French fries), fruit, nuts and soy } \\
\text { protein, cereal fiber, PUFA/SFA, } \\
\text { multivitamin use } \\
\text { - } \quad \text { Neutral components: Alcohol } \\
\text { - } \quad \text { Negative components: Trans fatty acids } \\
\text { Dietary assessment methods: } 124 \text {-item, } \\
\text { validated FFQ that captured dietary intake } \\
\text { during high school, (aged } 13-18 y \text { ) measured in } \\
\text { 1997 when subjects were } 411 y \\
\text { Outcome assessment methods: F/u with } \\
\text { study subjects, National Death Index, medical } \\
\text { records and pathology reports (Note: This paper } \\
\text { also examined various molecular subtypes of } \\
\text { breast cancer; results for molecular subtypes } \\
\text { were not extracted in this table) }\end{array}$ & $\begin{array}{l}\text { "Prudent" pattern was not significantly } \\
\text { associated with premenopausal breast } \\
\text { cancer after } 22 \mathrm{y} \text { f/u. } \\
\text { "Western" pattern was not significantly } \\
\text { associated with total or premenopausal } \\
\text { breast cancer after } 22 \mathrm{y} \text { f/u: } \\
\text { "Fast food" pattern was not significantly } \\
\text { associated with total or premenopausal } \\
\text { breast cancer after } 22 \mathrm{y} / \mathrm{u} \text { : } \\
\text { AHEI score was not significantly } \\
\text { associated with total or premenopausal } \\
\text { breast cancer after } 22 \mathrm{y} \text { f/u. }\end{array}$ & $\begin{array}{l}\text { - Did not account for missing data } \\
\text { - No preregistered statistical plan; potential } \\
\text { for selective outcome reporting } \\
\text { Funding Sources: NIH } \\
\text { Summary: In all women, higher adherence to } \\
\text { the "prudent" pattern during adolescence was } \\
\text { significantly associated with reduced risk of } \\
\text { breast cancer after } 22 y \text { flu. However, there } \\
\text { was no significant associated with risk of } \\
\text { premenopausal breast cancer risk. } \\
\text { Adherence to the "western", "fast food", and } \\
\text { AHEl patterns during adolescence was not } \\
\text { significantly associated with risk of total or } \\
\text { premenopausal breast cancer risk after } 22 y \\
\text { f/u. }\end{array}$ \\
\hline Harris, $2017^{13}$ & Dietary patterns: & Significant: & Key confounders accounted for: \\
\hline $\begin{array}{l}\text { PCS (Nurses' Health } \\
\text { Study II) }\end{array}$ & $\begin{array}{l}\text { Adherence to a dietary pattern identified via } \\
\text { reduced rank regression (response variables: C- } \\
\text { reactive protein, IL6, and TNFa receptor 2), } \\
\text { categorical (quintiles): }\end{array}$ & $\begin{array}{l}\text { Adolescent "inflammatory" pattern and } \\
\text { premenopausal breast cancer after } 22 \mathrm{y} \text { f/u: } \\
\text { - Q1, } \mathrm{n}=162 \text { : HR: } 1.00 \\
\text { - Q2, n=154: HR: } 0.96,95 \% \mathrm{Cl}: \\
\quad 0.77,1.20\end{array}$ & $\begin{array}{l}\text { Sex, age, alcohol intake (in adults), physical } \\
\text { activity, height at } 18 \mathrm{y}, \mathrm{BMl} \text { at } 18 \mathrm{y} \text {, weight } \\
\text { change since } 18 \mathrm{y} \text {, family history of the cancer }\end{array}$ \\
\hline
\end{tabular}

xvi McCullough, M.L. et al. (2002) Diet quality and major chronic disease risk in men and women: moving toward improved dietary guidance. Am. J. Clin. Nutr., 76, 1261-1271. 


\section{Study and \\ Participant \\ Characteristics \\ Intervention/Exposure and Outcomes \\ - "Inflammatory" dietary pattern: Higher loadings for sugar-sweetened and diet soft drinks, refined grains, red and processed meat, margarine, corn, other vegetables, and fish, and lower in green leafy vegetables, yellow vegetables, cruciferous vegetables, and coffee \\ Subjects were $100 \%$ female, $\sim 41$ yo in 1997 , $\sim 21 \mathrm{~kg} / \mathrm{m} 2$ at $18 \mathrm{y}$,}

Results

Excluded subjects who did not complete the high school FFQ, implausible energy intake, missing data, prevalent cancer before 1989

( $61 \%$ of the original sample)

\section{Dietary assessment methods:}

Adolescence: 124 -item, validated FFQ that captured dietary intake during high school, (aged 13-18y) measured in 1997 when subjects were $\sim 41 \mathrm{y}$

Early adulthood: 130-item, validated FFQ, measured in 1991 when subjects were 27-44y

Outcome assessment methods: F/u with study subjects, National Death Index, medical records and pathology reports (Note: This paper also examined various molecular subtypes of breast cancer; results for molecular subtypes were not extracted in this table)
- Q3, n=180: HR: 0.96, 95\% Cl: $0.77,1.20$

- Q4, n=189: HR: $1.30,95 \% \mathrm{Cl}$ : $1.04,1.62$

- Q5, n=185: HR: $1.35,95 \% \mathrm{Cl}$ : $1.06,1.73$

- $p$-trend $=0.002$

Average of adolescent and early adult "inflammatory" pattern and total breast cancer after 22y f/u:

- Q1, n=263: HR: 1.00

- Q2, n=295: HR: $1.15,95 \% \mathrm{Cl}$ : $0.97,1.36$

- Q3, n=301: HR: $1.22,95 \% \mathrm{Cl}$ : $1.03,1.44$

- Q4, n=270: HR: $1.13,95 \% \mathrm{Cl}$ : $0.94,1.35$

- Q5, n=270: HR: $1.25,95 \% \mathrm{Cl}$ : $1.03,1.52$

- $\mathrm{p}$-trend $=0.04$

\section{Non-Significant:}

Adolescent "inflammatory" pattern was not significantly associated with total or postmenopausal breast cancer after 22y f/u.

Results were similar when the early adulthood "inflammatory" pattern was analyzed, and when adolescent and early adult "inflammatory" patterns were
Confounding, Study Limitations, and Summary of Findings

outcome, hormonal contraceptive, menopausal status

\section{Other:}

Total energy intake, age at menarche, age at first birth, parity, history of benign breast disease, hormone replacement therapy

\section{Limitations:}

- Did not account for race/ethnicity, socioeconomic status, smoking

- Only assessed dietary intake twice during f/u; did not account for possible changes in dietary intake over $\mathrm{f} / \mathrm{u}$

- Did not account for missing data

- No preregistered statistical plan; potential for selective outcome reporting

\section{Funding Sources: NIH}

Summary: Higher adherence to an "inflammatory" dietary pattern during adolescence wass signiciantly associated with increased risk of premenopausal breast cancer after $22 y \mathrm{f} / \mathrm{u}$. However, adherence to the "inflammatory" dietary pattern was not significantly associated with total or postmenopausal breast cancer risk

Results were similar when the early adulthood "inflammatory" pattern was analyzed, and when adolescent and early adult "inflammatory" patterns were averaged, except for the results for all cases of breast cancer. 


\begin{tabular}{|c|c|c|c|}
\hline & Intervention/Exposure and Outcomes & Results & $\begin{array}{l}\text { Confounding, Study Limitations, and } \\
\text { Summary of Findings }\end{array}$ \\
\hline & & $\begin{array}{l}\text { averaged, except for the results for all } \\
\text { cases of breast cancer. }\end{array}$ & $\begin{array}{l}\text { When "inflammatory" dietary pattern was } \\
\text { calculated based on an average of } \\
\text { adolescence and young adulthood, higher } \\
\text { adherence was significantly associated with } \\
\text { increases risk of total breast cancer after } 22 y \\
\text { f/u. }\end{array}$ \\
\hline $\begin{array}{l}\text { Kane-Diallo, } \mathbf{2 0 1 8}^{14} \\
\text { PCS (NutriNet-Sante } \\
\text { study) } \\
\text { France }\end{array}$ & \multirow{2}{*}{$\begin{array}{l}\text { Dietary patterns: } \\
\text { "Pro plant-based" dietary score, categorical } \\
\text { (tertiles) } \\
\text { - Higher in plant foods: vegetables, legumes, } \\
\text { fruits, cereal products, potatoes, nuts, } \\
\text { vegetables oils } \\
\text { - Lower in animal foods: red and processed } \\
\text { meat, eggs, animal fat, dairy products, } \\
\text { seafood }\end{array}$} & \multirow{2}{*}{$\begin{array}{l}\text { Significant: N/A } \\
\text { Non-Significant: } \\
\text { "Pro plant-based" dietary score at } 49 \mathrm{y} \text { was } \\
\text { not significantly associated with risk of } \\
\text { total, premenopausal, or postmenopausal } \\
\text { breast cancer after } 4.3 y \text { f/u. }\end{array}$} & $\begin{array}{l}\text { Key confounders accounted for: } \\
\text { Sex, age, education, alcohol intake (in adults), } \\
\text { physical activity, smoking, height, BMI, family } \\
\text { history of the cancer outcome, hormonal } \\
\text { contraceptive, menopausal status }\end{array}$ \\
\hline Subjects were $100 \%$ & & & \multirow{3}{*}{$\begin{array}{l}\text { Other: Energy intake without alcohol, number } \\
\text { of 24-hr dietary records, Lipids intake, } \\
\text { Hormone replacement therapy, Number of } \\
\text { children } \\
\text { Limitations: } \\
\text { - Did not account for race/ethnicity } \\
\text { - Only assessed dietary intake only during } \\
\text { first } 2 y \text { of f/u; did not account for possible } \\
\text { changes in dietary intake over f/u } \\
\text { - Did not account for missing data } \\
\text { - No preregistered statistical plan; potential } \\
\text { for selective outcome reporting }\end{array}$} \\
\hline $\begin{array}{l}\text { temale, } 449 \mathrm{y}(\text { all } \\
>45 \mathrm{y}), \sim 25 \mathrm{~kg} / \mathrm{m} 2 \\
\sim 44 \% \text { never-smokers, } \\
9.7 \mathrm{~g} / \mathrm{d} \text { alcohol }\end{array}$ & $\begin{array}{l}\text { Dietary assessment methods: } 3,24 \text {-hour } \\
\text { dietary recalls, assessed every } 6 \text { mo during the } \\
\text { first } 2 y \text { of f/u, at age } \sim 49 y\end{array}$ & & \\
\hline \multirow{2}{*}{$\begin{array}{l}\text { Excluded those with } \\
\text { prevalent cancer; }<3 \\
24-\mathrm{hr} \text { recalls within the } \\
\text { first year of f/u; } \\
\text { missing } \mathrm{f} / \mathrm{u} \text { data; } \\
\text { implausible energy } \\
\text { intake; }<45 \mathrm{y}(79 \% \text { of } \\
\text { original sample) }\end{array}$} & \multirow[t]{2}{*}{$\begin{array}{l}\text { Outcome assessment methods: } \\
\text { Participant report, medical record review, } \\
\text { pathological reports }\end{array}$} & & \\
\hline & & & $\begin{array}{l}\text { Funding Sources: Ministere de la Sante, } \\
\text { Institut de Veille Sanitaire, Institut National de } \\
\text { la Prevention et de l'Education pour la Sante, } \\
\text { Region Ile-de-France, Institut National de la } \\
\text { Sant_e et de la Recherche Medicale, Institut } \\
\text { National de la Recherche Agronomique, } \\
\text { Conservatoire National des Arts et Metiers, } \\
\text { The French National Cancer Institute }\end{array}$ \\
\hline
\end{tabular}




\section{Kojima, $2017^{15}$}

\section{PCS (Japan}

Collaborative Cohort

Study)

Japan

Analytic N: 23172

Subjects were $100 \%$ female, $~ 55 y$ o, $~ 70 \%$ normal weight, $\sim 88 \%$ never smokers, $\sim 74 \%$ currently drink alcohol

Excluded men, those with prevalant cancer, stroke, CVD, or diabetes, missing dietary data, missing outcome data, implausible energy intake, or died within first $5 y$ of $\mathrm{f} / \mathrm{u}(\sim 79 \%$ of the original sample)

\section{Dietary patterns:}

Adherence to 3 dietary patterns identified using factor analysis, categorical (tertiles):

- "Vegetable pattern": Higher loadings for vegetables, potatoes, seaweed, tofu, fruits, fresh fish, eggs, and miso soup

- "Animal food pattern": Higher loadings for meat, deep-fried foods, fried vegetables, fish paste and salt-preserved fish

- "Dairy product pattern": Higher loadings for milk, dairy products, fruits, coffee and tea

Dietary assessment methods: 39-item, validated $F F Q$, at baseline,

Outcome assessment methods: Populationbased cancer registries, death record review

\section{Significant:}

"Animal food pattern" at 55y and

premenopausal breast cancer after $16.9 \mathrm{y}$ f/u:

- T1, n=20: HR: 1.00

- T2, n=13: HR: $0.47,95 \% \mathrm{Cl}: 0.22$, 1.00

- T3, n=15: HR: 0.42, 95\% Cl: 0.18, 0.93

- $\quad \mathrm{p}$-trend $=0.04$

\section{Non-Significant:}

"Animal food pattern" at 55y was not significantly associated with postmenopausal breast cancer after $16.9 \mathrm{y}$ f/u.

"Vegetable pattern" at 55y was not significantly associated with premenopausal or postmenopausal breast cancer after $16.9 \mathrm{y} / \mathrm{u}$.

"Dairy pattern" at 55y was not significantly associated with premenopausal or
Summary: "Pro plant-based" dietary score at $49 y$ was not significantly associated with risk of breast cancer, including pre- and postmenopausal breast cancer, after $4.3 y \mathrm{f} / \mathrm{u}$.

\section{Key confounders accounted for:}

Sex, age, education, alcohol intake (in adults), physical activity, smoking, BMI, family history of the cancer outcome, menopausal status

\section{Other:}

Area, age at Menarche, age at first birth, parity, energy intake, hormone therapy

\section{Limitations:}

- Did not account for race/ethnicity, hormonal contraceptive

- Excluded those with chronic diseases at baseline

- Only assessed dietary intake once at baseline; did not account for possible changes in dietary intake over f/u

- Did not account for missing data

- No preregistered statistical plan; potential for selective outcome reporting

\section{Funding Sources: NR}

Summary: Higher "animal food pattern" adherence at 55y was significantly associated with increased premenopausal breast cancer risk after 16.9y f/u. "Animal food pattern" 


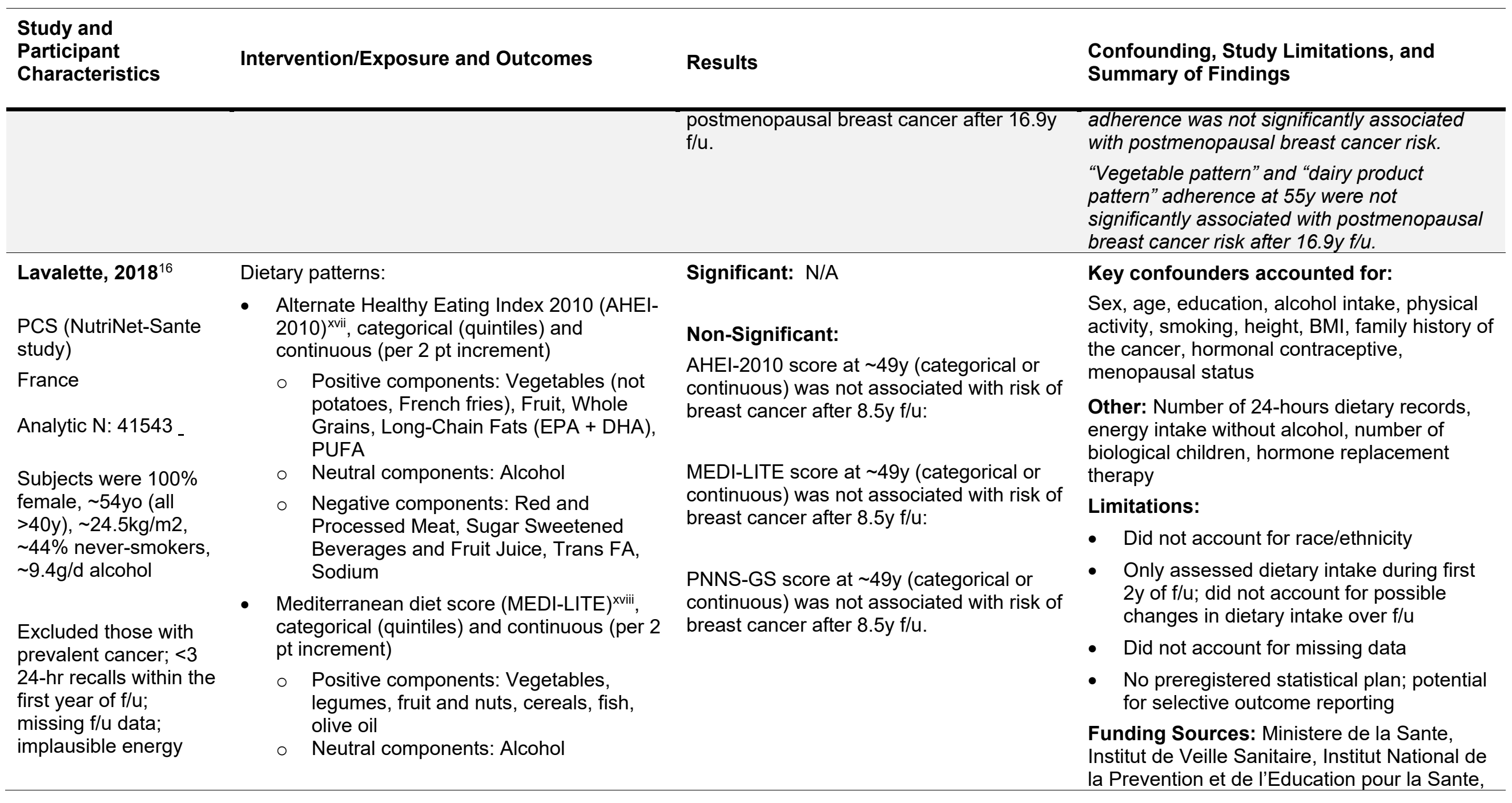

xvii Chiuve SE, Fung TT, Rimm EB, Hu FB, McCullough ML, Wang M, et al. Alternative dietary indices both strongly predict risk of chronic disease. J Nutr 2012;142:1009-18.

xviii Sofi F, Macchi C, Abbate R, Gensini GF, Casini A. Mediterranean diet and health status: an updated meta-analysis and a proposal for a literature-based adherence score. Public Health Nutr 2014;17: 2769-82. 


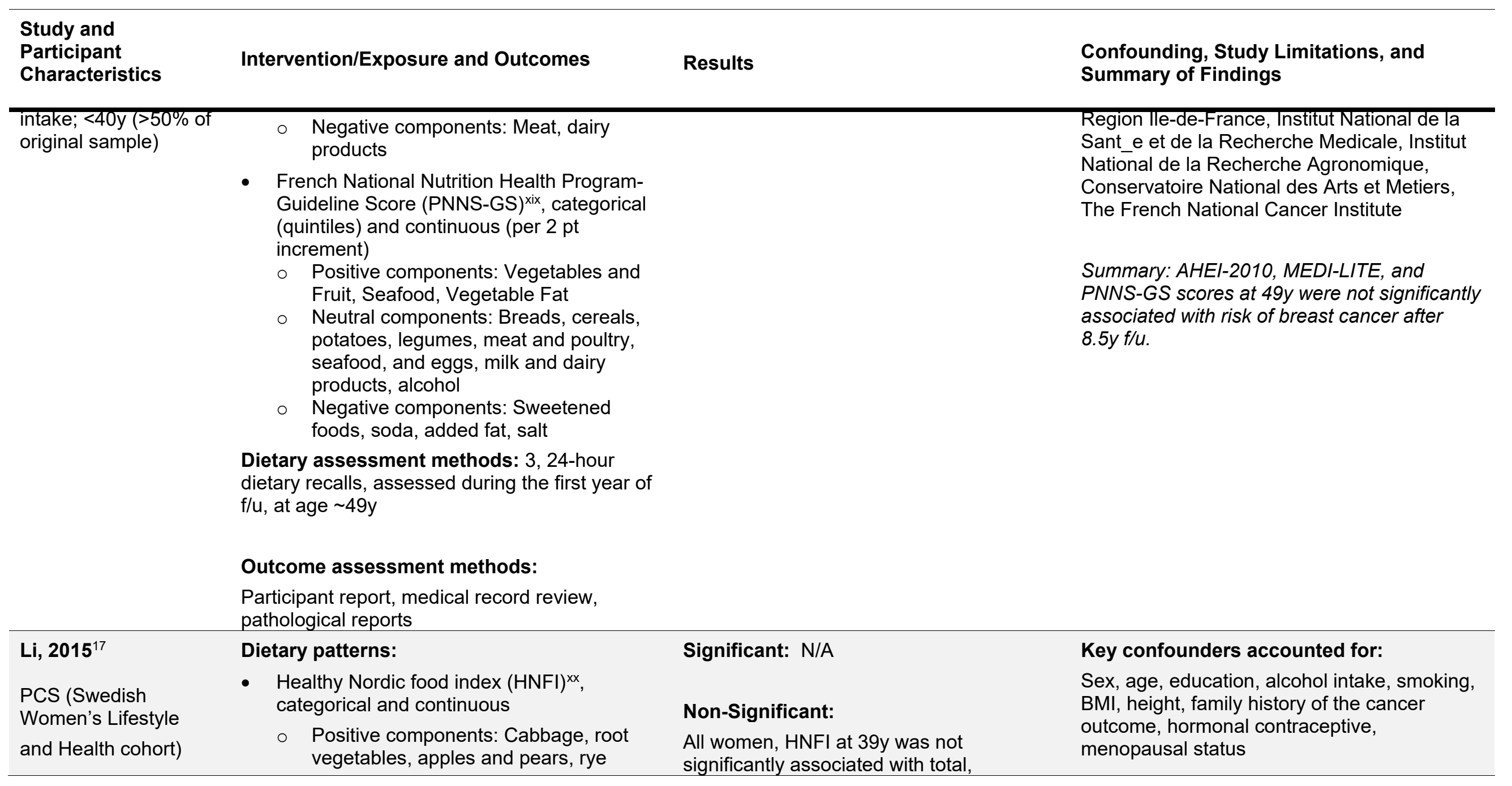

xix Estaquio C, Kesse-Guyot E, Deschamps V, Bertrais S, Dauchet L, Galan P, et al. Adherence to the French Programme National Nutrition Sante Guideline Score is associated with better nutrient intake and nutritional status. J Am Diet Assoc 2009;109:1031-41.

xx Olsen A, Egeberg R, Halkjær J, Christensen J, Overvad K, Tjønneland A (2011) Healthy aspects of the Nordic diet are related to lower total mortality. J Nutr 141:639-644 


\section{Study and \\ Participant}

Characteristics

Intervention/Exposure and Outcomes

\section{Results} cancer after $20 \mathrm{y} / \mathrm{u}$.

\section{Analytic N: 44296}

bread, oatmeal, fish

Dietary assessment methods: 8-item, validated $F F Q$, at baseline, $\sim 39 \mathrm{y}$

Subjects were $100 \%$ female, 39yo (29$49 \mathrm{y}), \sim 23 \mathrm{~kg} / \mathrm{m} 2, \sim 40 \%$ never smokers, $\sim 2.9 \mathrm{~g} / \mathrm{d}$ alcohol

Exclude those with previous breast cancer, extreme energy intakes, missing data on any variable $(\sim 10 \%$ of original sample)

\section{Outcome assessment methods: Swedish}

Cancer Registry and Cause of Death Registry (Note: This paper also examined various molecular subtypes of breast cancer; results for molecular subtypes were not extracted in this table)

\section{McKenzie, $2015^{18}$}

PCS (European

Prospective

Investigation into

Cancer and Nutrition

Cohort Study)

Denmark, France,

Germany, Greece,

\section{Dietary patterns:}

- Diet score, categorical (quintiles)

- Positive components: Cereal fiber, folate, PUFA/SFA, fatty fish, fruits and vegetables

- Negative components: margarine, glycemic load

Dietary assessment methods: FFQ, diet premenopausal, or postmenopausal breast

Confounding, Study Limitations, and Summary of Findings

\section{Other:}

Age at menarche, history of benign breast disease, age at first child, parity,

breastfeeding, saturated fat intake, energy intake

\section{Limitations:}

- Did not account for race/ethnicity, physical activity

- Only assessed dietary intake once at baseline; did not account for possible changes in dietary intake over $\mathrm{f} / \mathrm{u}$

- Did not account for missing data

- No preregistered statistical plan; potential for selective outcome reporting

Funding Sources: Swedish Cancer Society, Swedish Research Council

Summary: HFNI score at $39 y$ was not significantly associated with risk of breast cancer (total, pre- and post-menopausal) after $20 y$ f/u.

\section{Key confounders accounted for:}

\section{Significant:}

Diet score at 53y and postmenopausal breast cancer $(n=7756)$ after $10.9 \mathrm{y} / \mathrm{u}$ :

- Q1, n=88: HR: 1.00

- Q2, n=93: HR: 0.93, 95\% Cl: 0.87 , 0.99

○ Q3, n=87: HR: $0.98,95 \%$ Cl: 0.91 1.06
Sex, age, education, alcohol intake (in adults) physical activity, smoking, height, BMI, hormonal contraceptive, menopausal status

Other:

Study center, age at menarche, age at first pregnancy, hormone replacement therapy, breastfeeding, energy intake without alcohol 


\begin{tabular}{|c|c|c|c|}
\hline $\begin{array}{l}\text { Study and } \\
\text { Participant } \\
\text { Characteristics }\end{array}$ & Intervention/Exposure and Outcomes & Results & $\begin{array}{l}\text { Confounding, Study Limitations, and } \\
\text { Summary of Findings }\end{array}$ \\
\hline $\begin{array}{l}\text { Italy, the Netherlands, } \\
\text { Norway, Spain, } \\
\text { Sweden and the } \\
\text { United Kingdom } \\
\text { Analytic N: } 242918 \\
\text { Subjects were } 100 \% \\
\text { female, } \sim 53 y 0, \\
\sim 25 \mathrm{~kg} / \mathrm{m} 2, \sim 49 \% \text { ever } \\
\text { smokers, } \sim 4.2 \mathrm{~g} / \mathrm{d} \\
\text { alcohol } \\
\\
\text { Exclude subjects with } \\
\text { prevalent cancer, } \\
\text { premenopausal, } \\
\text { missing f/u data, top or } \\
\text { bottom } 1 \% \text { of energy } \\
\text { intake to energy } \\
\text { requirement, missing } \\
\text { covariate data }(\sim 44 \% \\
\text { of original sample) }\end{array}$ & $\begin{array}{l}\text { history questionnaires, validated, at baseline, } \\
53 y \\
\text { Outcome assessment methods: Cancer } \\
\text { registries, health insurance records, } \\
\text { cancer/pathology registrations and through } \\
\text { participants and next-of-kin }\end{array}$ & $\begin{aligned} & \circ \text { Q4, } n=98: H R: 0.89,95 \% \mathrm{Cl}: 0.83 \text {, } \\
& 0.95 \\
& \circ \text { Q5, } n=122: H R: 0.90,95 \% \mathrm{Cl}: \\
& 0.84,0.97 \\
& \text { p-trend }=0.005 \\
& \\
& \text { Non-Significant: N/A }\end{aligned}$ & $\begin{array}{l}\text { Limitations: } \\
\text { - Did not account for race/ethnicity, family } \\
\text { history of the cancer outcome } \\
\text { - Only assessed dietary intake once at } \\
\text { baseline; did not account for possible } \\
\text { changes in dietary intake over f/u } \\
\text { - Did not account for missing data } \\
\text { - } \quad \text { No preregistered statistical plan; potential } \\
\quad \text { for selective outcome reporting } \\
\text { Funding Sources: Genesis Oncology Trust; } \\
\text { European Commission the International } \\
\text { Agency for Research on Cancer; Danish } \\
\text { Cancer Society; Ligue Contre le Cancer, } \\
\text { Institut Gustave Roussy, Mutuelle Generale } \\
\text { de l'Education Nationale, Institut National de } \\
\text { la Sante et de la Recherche Medicale; } \\
\text { Deutsche Krebshilfe, Deutsches } \\
\text { Krebsforschungszentrum and Federal Ministry } \\
\text { of Education and Research; Hellenic Health } \\
\text { Foundation; Italian Association for Research } \\
\text { on Cancer and National Research Council; } \\
\text { Dutch Ministry of Public Health, Welfare and } \\
\text { Sports, Netherlands Cancer Registry, LK } \\
\text { Research Funds, Dutch Prevention Funds, } \\
\text { Dutch ZON, World Cancer Research Fund, } \\
\text { Statistics Netherlands; Nordforsk, Nordic } \\
\text { Centre of Excellence programme on Food, } \\
\text { Nutrition and Health (Norway); Health } \\
\text { Research Fund of the Spanish Ministry of } \\
\text { Health, the Catalan Institute of Oncology, and } \\
\text { the participating regional governments and } \\
\text { institutions of Spain; Swedish Cancer Society, } \\
\text { Swedish Scientific Council and Regional }\end{array}$ \\
\hline
\end{tabular}




\section{Study and \\ Participant \\ Characteristics}

Results

Confounding, Study Limitations, and Summary of Findings

Government of Skane and Vasterbotten; Cancer Research United Kingdom, Medical Research Council, Stroke Association, British Heart Foundation, Department of Health,

Food Standards Agency, and Welcome Trust

Summary: Higher diet score at 53y was significantly associated with decreased risk of postmenopausal breast cancer after $10.9 y \mathrm{f} / \mathrm{u}$.

\section{Key confounders accounted for:}

Subjects were $100 \%$ female, 38 yo (21 $69 y), \sim 50 \%$

overweight/obese, $\sim 68 \%$ never smokers $\sim 77 \%<1$ drink/wk

\section{Excluded those not} 21-69y at baseline incomplete

questionnaires, with prevalent cancer, pregnant at baseline, implausible energy
Dietary patterns:

- WCRF/AICR Score, diet only, categorical (tertiles) and continuous (per 0.5 unit increase)

- Positive components: Vegetables and fruit, dietary fiber

- Negative components: Red and processed meat, sugary drinks, alcohol, sodium, energy-dense foods

\section{Significant:}

WCRF/AICR score, diet only, time-varying, continuous (per 0.5 unit increase), $n=1766$ HR:0.91, 95\% Cl: 0.83, 0.99; p-trend=0.04

When results were analyzed by

menopausal status, results were borderline significant in premenopausal breast cance $(p=0.06)$, and non-significant for postmenopausal breast cancer.

Dietary assessment methods: 68- and 85item FFQ at baseline (1995) and f/u (2001)

Outcome assessment methods: Self-report, linkage with cancer registries

\section{Non-Significant:}

WCRF/AICR score, diet only, at baseline (categorical, continuous, and time-varyingcategorical), 38yo, was not significantly associated with breast cancer after $13.9 \mathrm{y}$ f/u.

Results were similar when pre- and postmenopausal breast cancer were analyzed separately.
Sex, age, race/ethnicity, education, alcoho intake, smoking, BMI, family history of the cancer outcome, hormonal contraceptive, menopausal status

Other:

Geographic region of residence, energy intake, parity, hormone replacement therapy

\section{Limitations:}

- Did not account for physical activity

- Only assessed dietary intake once at baseline; did not account for possible changes in dietary intake over f/u

- Did not account for missing data

- $\quad$ No preregistered statistical plan; potential for selective outcome reporting

\section{Funding Sources: $\mathrm{NIH}$}

Summary: Adherence to the WCRF/AICR diet score at $38 y$ was not significantly associated 


\begin{tabular}{|c|c|c|c|}
\hline $\begin{array}{l}\text { Study and } \\
\text { Participant } \\
\text { Characteristics }\end{array}$ & Intervention/Exposure and Outcomes & Results & $\begin{array}{l}\text { Confounding, Study Limitations, and } \\
\text { Summary of Findings }\end{array}$ \\
\hline $\begin{array}{l}\text { intake }(\sim 17 \% \text { of } \\
\text { original sample) }\end{array}$ & & & $\begin{array}{l}\text { with risk of breast cancer after 13.9y f/u when } \\
\text { analyzed categorically or continuously. In } \\
\text { addition, adherence to the WCRF/AICR diet } \\
\text { score was also not significantly associated } \\
\text { with breast cancer risk when analyzed } \\
\text { categorically, time-varying. } \\
\text { However, higher WCRF/AICR score, diet only, } \\
\text { time-varying, analyzed continuously, was } \\
\text { associated with significantly decreased risk of } \\
\text { breast cancer after } 13.9 y \text { f/u. }\end{array}$ \\
\hline $\begin{array}{l}\text { Penniecook- } \\
\text { Sawyers, 2016 } \\
\text { PCS (Adventist Health } \\
\text { Study-2) } \\
\text { United States } \\
\text { Analytic N: } 50404 \\
\text { Subjects were } 100 \% \\
\text { female, } 64 \mathrm{y}(35- \\
110 \mathrm{y}), \sim 27.5 \mathrm{~kg} / \mathrm{m} 2 \text {, } \\
\sim 15 \% \text { ever smokers, } \\
\sim 11 \% \text { drank alcohol } \\
\text { within } 2 \mathrm{y} \text { of enrollment } \\
\text { Excluded Canadian } \\
\text { participants, those } \\
\text { with prevalent cancer, } \\
\text { age <35 years, }\end{array}$ & $\begin{array}{l}\text { Dietary patterns: } \\
\text { - "Vegans"xxi: Red meat, poultry, fish; eggs; } \\
\text { and dairy <1 time/mo } \\
\text { "Lacto-ovo-vegetarian": Red meat, poultry, } \\
\text { and fish <1 time/mo, eggs or dairy }>1 \\
\text { time/mo } \\
\text { "Pesco-vegetarian": Red meat or poultry }<1 \\
\text { time/mo, fish }>1 \text { time/mo, and eggs/dairy in } \\
\text { any amount } \\
\text { "Semi-vegetarian": Red meat or poultry }>1 \\
\text { time/mo, and all meats combined (including } \\
\text { fish) }<1 \text { time/wk and eggs/dairy in any } \\
\text { amount } \\
\text { "Non-vegetarians": Red meat and poultry } \\
>1 \text { time/mo and all meats combined } \\
\text { (including fish) }>1 \text { time/wk, and eggs/dairy } \\
\text { in any amount } \\
\text { "Vegetarians" vs. "nonvegetarians" consumed } \\
\text { higher amounts of fruits, vegetables, avocados, } \\
\text { non-fried potatoes, whole grains, legumes, soya }\end{array}$ & $\begin{array}{l}\text { Significant: N/A } \\
\text { Non-Significant: } \\
\text { "Vegetarian" pattern at } 64 \mathrm{y} \text { was not } \\
\text { significantly associated with total or } \\
\text { postmenopausal breast cancer after } 7.8 y \\
\text { f.u. Results were similar when analyzed by } \\
\text { non-black or black race. }\end{array}$ & $\begin{array}{l}\text { Key confounders accounted for: } \\
\text { Sex, age, race/ethnicity, education, alcohol } \\
\text { intake, physical activity, smoking, height, BMI, } \\
\text { family history of the cancer outcome, } \\
\text { hormonal contraceptive, menopausal status } \\
\text { Other: } \\
\text { Mammography in last } 2 y \text { after } 42 y \text {, age at } \\
\text { menarche, hormone replacement therapy, } \\
\text { age at first child, number of children, } \\
\text { Breastfeeding } \\
\text { Limitations: } \\
\text { - Only assessed dietary intake once at } \\
\text { baseline; did not account for possible } \\
\text { changes in dietary intake over f/u } \\
\text { - Did not account for missing data } \\
\text { - No preregistered statistical plan; potential } \\
\text { for selective outcome reporting }\end{array}$ \\
\hline
\end{tabular}

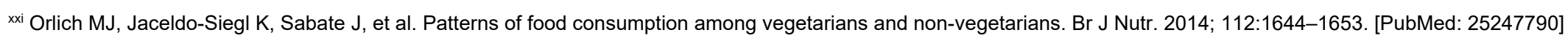




\begin{tabular}{|c|c|c|c|}
\hline $\begin{array}{l}\text { Study and } \\
\text { Participant } \\
\text { Characteristics }\end{array}$ & Intervention/Exposure and Outcomes & Results & $\begin{array}{l}\text { Confounding, Study Limitations, and } \\
\text { Summary of Findings }\end{array}$ \\
\hline \multirow{3}{*}{$\begin{array}{l}\text { incomplete FFQ, } \\
\text { implausible energy } \\
\text { intakes, never had } \\
\text { menstrual period } \\
(\sim 20 \% \text { of original } \\
\text { sample) }\end{array}$} & \multicolumn{2}{|l|}{$\begin{array}{l}\text { foods, nuts and seeds, and observed among } \\
\text { vegetarians, was lower amounts of meats, dairy } \\
\text { products, eggs, refined grains, added fats, } \\
\text { sweets, snack foods and non-water beverages }\end{array}$} & $\begin{array}{l}\text { +Funding Sources: NIH, World Cancer } \\
\text { Research Fund }\end{array}$ \\
\hline & \multicolumn{2}{|l|}{$\begin{array}{l}\text { Dietary assessment methods: }>220 \text {-item, } \\
\text { validated FFQ, at baseline, }>30 y\end{array}$} & $\begin{array}{l}\text { Summary: Consuming various "vegetarian" } \\
\text { patterns at } 64 y \text { was not significantly } \\
\text { associated with risk of total or premenopausal } \\
\text { breast cancer after } 7.8 y \text { f/u. }\end{array}$ \\
\hline & \multicolumn{2}{|l|}{$\begin{array}{l}\text { Outcome assessment methods: State cancer } \\
\text { registries, patient f/u with medical record } \\
\text { verification }\end{array}$} & $\begin{array}{l}\text { Consuming a "semi-vegetarian" pattern vs. a } \\
\text { "non-vegetarian" pattern at } 64 y \text { was } \\
\text { associated with significantly increased risk of } \\
\text { postmenopausal breast cancer after } 7.8 \mathrm{y} \text { f/u. }\end{array}$ \\
\hline Petimar, 201921 & Dietary patterns: & Significant: & Key confounders accounted for: \\
\hline PCS (Sister Study) & $\begin{array}{l}\text { - Alternative Mediterranean Diet Score } \\
(\text { aMED) })^{x \times i i}, \text { categorical (quintiles) }\end{array}$ & $\begin{array}{l}\text { DASH score at } 55 y \text { and total invasive } \\
\text { breast cancer after } 7.6 \mathrm{y} / \mathrm{u} \text { : }\end{array}$ & \multirow{2}{*}{$\begin{array}{l}\text { Sex, age, race/ethnicity, income, education, } \\
\text { alcohol intake, physical activity, smoking, BMI, } \\
\text { family history of the cancer outcome, } \\
\text { hormonal contraceptive, menopausal status } \\
\text { Other: }\end{array}$} \\
\hline & - Positive components: Vegetables (not & - $\quad$ Q1, n=388: HR: 1.00 & \\
\hline \multirow{4}{*}{$\begin{array}{l}\text { Subjects were } 100 \% \\
\text { female, } \sim 55 \mathrm{yo}, \\
\sim 27 \mathrm{~kg} / \mathrm{m} 2, \sim 45 \% \text { ever } \\
\text { smokers, } \sim 5.5 \mathrm{~g} / \mathrm{d} \\
\text { alcohol }\end{array}$} & $\begin{array}{ll} & \text { Neutral components: Alcohol } \\
& \text { Negative components: Red and }\end{array}$ & $0.77,1.03$ & \multirow{5}{*}{$\begin{array}{l}\text { Total energy intake, age at first live birth, } \\
\text { parity, hormone replacement therapy, age at } \\
\text { menarche, breastfeeding, time of last } \\
\text { mammogram } \\
\text { Limitations: } \\
\text { - Only assessed dietary intake once at } \\
\text { baseline; did not account for possible } \\
\text { changes in dietary intake over f/u }\end{array}$} \\
\hline & \multirow{3}{*}{$\begin{array}{l}\text { processed meat } \\
\text { DASH Index } \\
\text { Poxiii, categorical (quintiles) } \\
\text { potatoes), nuts and legumes, fruit and } \\
\text { fruit juice, whole grains, low-fat dairy }\end{array}$} & $\begin{array}{l}\text { - } Q 4, n=417: \text { HR: } 0.78,95 \% \mathrm{Cl}: \\
0.67,0.90\end{array}$ & \\
\hline & & - $\quad p$-trend $=0.001$ & \\
\hline & & \multirow{2}{*}{ Non-Significant: } & \\
\hline $\begin{array}{l}\text { Excluded those with } \\
\text { prevalent cancer, }\end{array}$ & $\begin{array}{l}\text { Negative components: Red and } \\
\text { processed meat, sweetened }\end{array}$ & & \\
\hline
\end{tabular}

xxii Fung TT, Hu FB, McCullough ML, Newby PK, Willett WC, Holmes MD. Diet quality is associated with the risk of estrogen receptor-negative breast cancer in postmenopausal women. J Nutr 2006;136:466-72.

xxiii Fung TT, Hu FB, Hankinson SE, Willett WC, Holmes MD. Lowcarbohydrate diets, dietary approaches to stop hypertension-style diets, and the risk of postmenopausal breast cancer. Am J Epidemiol 2011;174:652-60. 


\begin{tabular}{|c|c|c|c|}
\hline $\begin{array}{l}\text { Study and } \\
\text { Participant } \\
\text { Characteristics }\end{array}$ & Intervention/Exposure and Outcomes & Results & $\begin{array}{l}\text { Confounding, Study Limitations, and } \\
\text { Summary of Findings }\end{array}$ \\
\hline $\begin{array}{l}\text { pregnant, } \\
\text { breastfeeding, missing } \\
\text { dietary intake data, } \\
\text { extreme calorie intake, } \\
\text { cases diagnosed in } \\
\text { first year of f/u, } \\
\text { missing covariate data } \\
(\sim 10 \% \text { of original } \\
\text { sample) }\end{array}$ & 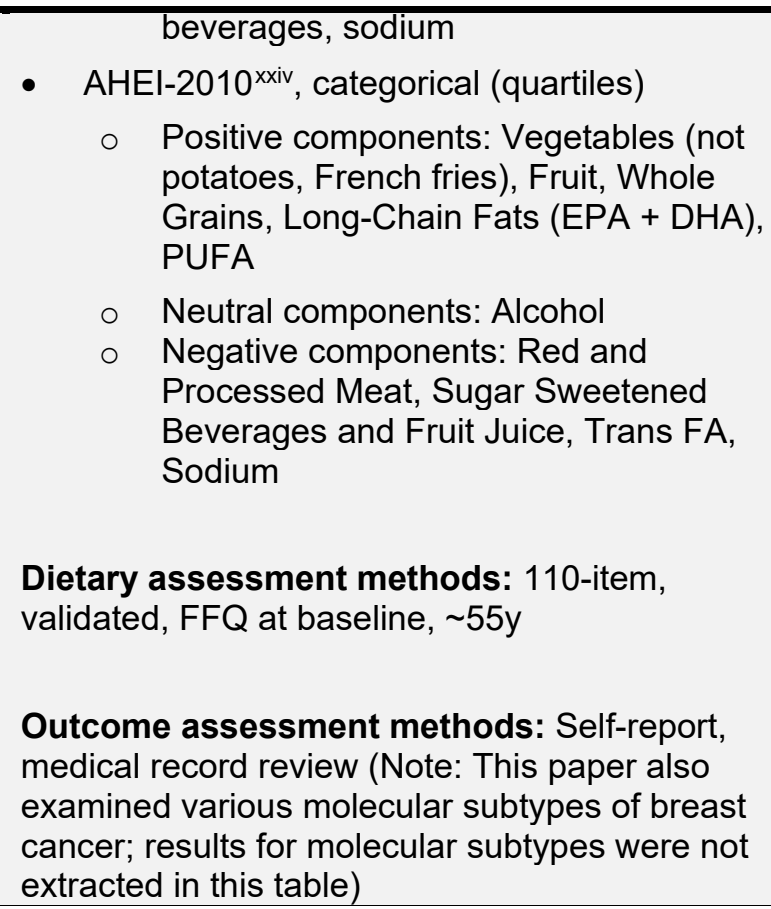 & $\begin{array}{l}\text { AHEI-2010 score, with and without alcohol, } \\
\text { at } 55 \mathrm{y} \text { was not significantly associated with } \\
\text { total invasive breast cancer after } 7.6 \mathrm{y} \text { f/u. } \\
\text { aMED score, with and without alcohol, at } \\
55 \mathrm{y}, \text { was not significantly associated with } \\
\text { total invasive breast cancer after } 7.6 \mathrm{y} / \mathrm{u} \text {. }\end{array}$ & $\begin{array}{l}\text { - Did not account for missing data } \\
\text { - No preregistered statistical plan; potential } \\
\quad \text { for selective outcome reporting } \\
\text { Funding Sources: NIH, Breast Cancer } \\
\text { Research Foundation } \\
\text { Summary: Higher DASH score at 55y was } \\
\text { significantly associated with reduced risk of } \\
\text { total invasive breast cancer after } 7.6 y \text { f/u. } \\
\text { AHEI-2010 and aMED scores, with and } \\
\text { without alcohol, at } 55 y, \text { were not significantly } \\
\text { associated with risk of total invasive breast } \\
\text { cancer after } 7.6 y \text { f/u. }\end{array}$ \\
\hline $\begin{array}{l}\text { Pot, } \mathbf{2 0 1 4}^{26} \\
\text { Nested Case-Control } \\
\text { Study (UK Dietary } \\
\text { Cohort Consortium) } \\
\text { United Kingdom }\end{array}$ & $\begin{array}{l}\text { Dietary patterns: } \\
\text { - Mediterranean Diet Score (MDS) })^{\times \times v} \text {, with } \\
\text { and without alcohol, categorical (tertiles) } \\
\circ \quad \text { Positive components: Vegetables, } \\
\text { legumes, fruit and nuts, cereals, fish, } \\
\text { MUFA/SFA } \\
\circ \quad \text { Neutral components: Alcohol }\end{array}$ & $\begin{array}{l}\text { Significant: } \\
\text { "High alcohol" pattern and breast cancer } \\
\text { ( } \mathrm{n}=387 \text { cases): } \\
\quad \text { T1: HR: } 1.00 \\
\text { - T2: HR: } 1.04,95 \% \mathrm{Cl}: 0.77,1.39 \\
\text { - T3: HR: } 1.28,95 \% \mathrm{Cl}: 0.95,1.71\end{array}$ & $\begin{array}{l}\text { Key confounders accounted for: } \\
\text { Sex, age, education, alcohol intake, physical } \\
\text { activity, weight, height, family history of the } \\
\text { cancer outcome, menopausal status } \\
\text { Other: }\end{array}$ \\
\hline
\end{tabular}

xxiv Chiuve SE, Fung TT, Rimm EB, Hu FB, McCullough ML, Wang M, Stampfer MJ, Willett WC. Alternative dietary indices both strongly predict risk of chronic disease. J Nutr 2012;142:1009-18.

xxv Trichopoulou A, Bamia C, Lagiou P, Trichopoulos D. Conformity to traditional Mediterranean diet and breast cancer risk in the Greek EPIC (European Prospective Investigation into Cancer and nutrition) cohort. Am J Clin Nutr. 2010; 92:620-5. [PubMed: 20631204] 


\section{Study and \\ Participant \\ Characteristics \\ Analytic N: 601 cases, 1891 controls \\ Subjects were $100 \%$ female, $~ 57$ yo, $\sim 25.7 \mathrm{~kg} / \mathrm{m} 2,60 \%$ never smokers, $\sim 9.5 \mathrm{~g} / \mathrm{d}$ alcohol \\ Cases were free from cancer at baseline, and were matched to 4 controls who were free of cancer at baseline and $\mathrm{f} / \mathrm{u}$ (matched on cohort, age at enrollment, and date of diet collection)}

Intervention/Exposure and Outcomes

Results

Confounding, Study Limitations, and Summary of Findings

○ Negative components: Red and processed meat, dairy products

- Three dietary patterns identified using principal components analyses: The first pattern had higher loadings for cheese, crisps and savory snacks, fresh fruit, legumes, low fat milk, nuts and seeds, other fruit, rice/pasta/other grains, sauces, vegetable mixed dishes, and lower in potatoes, poultry, and red meat. The other two patterns added little variation and were not included.

- Only two of the three dietary patterns identified using reduced rank regression were examined (response variables: alcohol, total fat, fiber):

○ "High alcohol": Higher loadings for wines, spirits, and beers and ciders

- "High fiber": Higher loadings for fiber, fresh fruit, raw and boiled vegetables, high fiber bread, high fiber breakfast cereals, lower in alcohol and total fat

Dietary assessment methods: 4-7 day dietary records, at baseline, $\sim 57 \mathrm{y}$
- $p$-trend $=0.08$

"High alcohol" pattern and postmenopausal breast cancer ( $\mathrm{n}=409$ cases):

- T1: HR: 1.00

- T2: HR: $1.14,95 \%$ Cl: 0.84, 1.55

- T3: HR: $1.46,95 \%$ Cl: 0.08, 1.98

- $\quad$-trend $=0.01$

\section{Non-Significant:}

MDS score, with and without alcohol, was not significantly associated with total or postmenopausal breast cancer.

PCA dietary pattern score was not significantly associated with total or postmenopausal breast cancer.

"High fiber" pattern score was not significantly associated with total or postmenopausal breast cancer.
Parity, hormone replacement therapy, breastfeeding

\section{Limitations:}

- Did not account for race/ethnicity, smoking, or hormonal contraceptive

- Only assessed dietary intake once at baseline; did not account for possible changes in dietary intake over $\mathrm{f} / \mathrm{u}$

- Did not account for missing data

- No preregistered statistical plan; potential for selective outcome reporting

\section{Funding Sources: NR}

Summary: MDS score, a factor analysis dietary pattern score, and a "high fiber" dietary pattern derived using RRR were not associated with risk of total or postmenopausal breast cancer.

Higher adherence to a "high alcohol" dietary pattern was associated with increased risk of total and postmenopausal breast cancer.

\section{Outcome assessment methods: NR}

\begin{tabular}{ll}
\hline Shin, 2016 & Dietary patterns: \\
PCS (Japan Public & $\begin{array}{l}\text { Adherence to } 3 \text { dietary patterns derived using } \\
\text { principal component analysis, categorical } \\
\text { Health Center-based }\end{array}$ \\
(quintiles):
\end{tabular}

\section{Significant:}

"Westernized" pattern score at 57y and breast cancer after 14.6y f/u:

- Q1, n=125: HR: 1.00
Key confounders accounted for:

Sex, age, race/ethnicity, alcohol intake, physical activity, smoking, BMI, menopausal status, use of exogenous hormones 


\begin{tabular}{|c|c|c|c|}
\hline $\begin{array}{l}\text { Study and } \\
\text { Participant } \\
\text { Characteristics }\end{array}$ & Intervention/Exposure and Outcomes & Results & $\begin{array}{l}\text { Confounding, Study Limitations, and } \\
\text { Summary of Findings }\end{array}$ \\
\hline $\begin{array}{l}\text { Prospective Study } \\
\text { (JPHC Study)) } \\
\text { Japan } \\
\text { Analytic N: } 49552 \\
\text { Subjects were } 100 \% \\
\text { female, } 57 \text { yo, } \\
23.5 \mathrm{kgm} / 2, \sim 94 \% \\
\text { never smokers } \\
\text { Exclude those with } \\
\text { prevalent cancer, } \\
\text { implausible energy } \\
\text { intakes, Non- } \\
\text { Japanese ethnicity, } \\
\text { late report of migration } \\
\text { occurring before the } \\
\text { start of the } \\
\text { study or incorrect birth } \\
\text { data), deceased or } \\
\text { moved out of study } \\
\text { area ( 31\% of original } \\
\text { sample) }\end{array}$ & $\begin{array}{l}\text { - } \quad \text { "Prudent" pattern: Higher loadings for } \\
\text { vegetables, fruits, soya products, potatoes, } \\
\text { seaweed, mushroom, and fish } \\
\text { - "Westernized" pattern: Higher loadings for } \\
\text { bread, meat, processed meats, dairy } \\
\text { products, soup, coffee, soft drinks, black } \\
\text { tea, sauces, mayonnaise and dressing } \\
\text { - } \quad \text { "Traditional Japanese" pattern: Higher } \\
\text { loadings for salmon, seafood other than } \\
\text { fish, oily fish, lean fish, salty fish, chicken } \\
\text { and pickles } \\
\text { Dietary assessment methods: } 147 \text {-item, } \\
\text { validated FFQ at baseline, } 57 y \\
\text { Outcome assessment methods: F/u with } \\
\text { subjects, population-based cancer registries } \\
\text { (Note: This paper also examined various } \\
\text { molecular subtypes of breast cancer; results for } \\
\text { molecular subtypes were not extracted in this } \\
\text { table) }\end{array}$ & 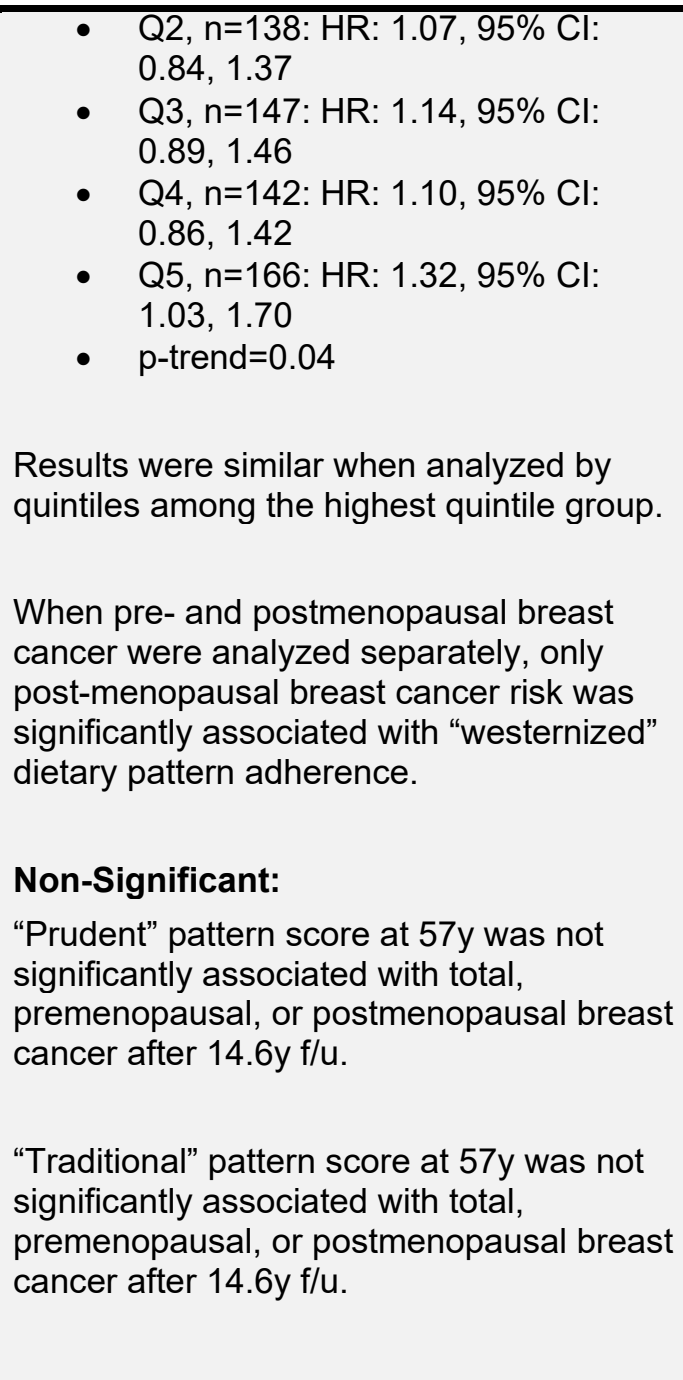 & $\begin{array}{l}\text { Other: } \\
\text { Public healthcare centre area, energy intake, } \\
\text { age at menarche, parity, age at first birth } \\
\text { Limitations: } \\
\text { - Did not account for socioeconomic status, } \\
\text { family history of the cancer outcome } \\
\text { - Only assessed dietary intake once at } \\
\text { baseline; did not account for possible } \\
\text { changes in dietary intake over f/u } \\
\text { - Did not account for missing data } \\
\text { - No preregistered statistical plan; potential } \\
\text { for selective outcome reporting } \\
\text { Funding Sources: National Cancer Center } \\
\text { Research and Development Fund, Ministry of } \\
\text { Health, Labour and Welfare of Japan } \\
\text { Summary: Higher adherence to a } \\
\text { "westernised" dietary pattern at } 57 y \text { was } \\
\text { significantly associated with increased risk of } \\
\text { breast cancer after } 14.6 y \text { flu. When pre- and } \\
\text { postmenopausal breast cancer were analyzed } \\
\text { separately, only postmenopausal breast } \\
\text { cancer risk was significantly associated with } \\
\text { "westernized" dietary pattern adherence. } \\
\text { Adherence to a "prudent" or "traditional" } \\
\text { Japanese dietary pattern at } 57 y \text { was not } \\
\text { significantly associated with risk of breast } \\
\text { cancer after } 14.6 y \text { flu. Results were similar } \\
\text { when pre- and postmenopausal breast cancer } \\
\text { were analyzed separately. }\end{array}$ \\
\hline
\end{tabular}




\begin{tabular}{|c|c|c|c|}
\hline $\begin{array}{l}\text { Study and } \\
\text { Participant } \\
\text { Characteristics }\end{array}$ & Intervention/Exposure and Outcomes & Results & $\begin{array}{l}\text { Confounding, Study Limitations, and } \\
\text { Summary of Findings }\end{array}$ \\
\hline $\begin{array}{l}\text { Subjects were } 100 \% \\
\text { female, } \sim 61 \mathrm{yo}(55- \\
69 \mathrm{y}), \sim 25 \mathrm{~kg} / \mathrm{m} 2, \sim 20 \% \\
\text { current smokers, } \\
\sim 26 \% 5-25 \mathrm{~g} / \mathrm{d} \text { alcohol } \\
\text { Excluded Cases and } \\
\text { subcohort with history } \\
\text { of cancer (except skin } \\
\text { cancer), incomplete or } \\
\text { inconsistent dietary } \\
\text { data }(\sim 33 \% \text { of original } \\
\text { sample) }\end{array}$ & $\begin{array}{l}\text { Dietary patterns: } \\
\text { - aMEDxxvi, with and without alcohol, } \\
\text { categorical and continuous } \\
\text { - } \quad \text { Positive components: Vegetables (not } \\
\text { potatoes), legumes, fruits, nuts, whole } \\
\text { grains, fish, MUFA/SFAs } \\
\text { - Neutral components: Alcohol } \\
\text { - Negative components: Red and } \\
\text { processed meat } \\
\text { - mMEDxvii, without alcohol, categorical and } \\
\text { continuous } \\
\text { - Positive components: Vegetables, } \\
\text { legumes, fruit, cereals, fish, } \\
\text { MUFA+PUFA/SFA } \\
\text { - Neutral components: Alcohol } \\
\text { - Negative components: Meat, dairy } \\
\text { products } \\
\text { WCRFxxviii, diet only, with and without } \\
\text { alcohol, continuous } \\
\text { - Positive components: Vegetables and } \\
\text { fruit, dietary fiber } \\
\text { Negative components: Red and } \\
\text { processed meat, sugary drinks, alcohol, } \\
\text { sodium, energy-dense foods } \\
\text { Dietary assessment methods: } 150-i t e m, \\
\text { validated FFQ at baseline, } ~ 61 y\end{array}$ & $\begin{array}{l}\text { Non-Significant: } \\
\text { aMED and mMED scores, with and without } \\
\text { alcohol, at } 61 y \text { was not significantly } \\
\text { associated with postmenopausal breast } \\
\text { cancer after } 20.3 y \text { f/u. } \\
\text { Results were similar when stratified by } \\
\text { years of f/u, age at baseline, smoking } \\
\text { status, alcohol intake, BMI, physical } \\
\text { activity, and family history of breast cancer. } \\
\text { aMED vs. WCRF, diet only, with and } \\
\text { without alcohol at } 61 y \text { was not significantly } \\
\text { associated with postmenopausal breast } \\
\text { cancer after } 20.3 y \text { f/u. }\end{array}$ & $\begin{array}{l}\text { Key confounders accounted for: } \\
\text { Sex, age, education, alcohol intake, physical } \\
\text { activity, smoking, height, BMI, family history of } \\
\text { the cancer outcome, hormonal contraceptive, } \\
\text { menopausal status } \\
\text { Other: } \\
\text { History of benign breast disease, age at } \\
\text { menarche, parity, age at first birth, hormone } \\
\text { replacement therapy } \\
\text { Limitations: } \\
\text { - Did not account for race/ethnicity } \\
\text { - Only assessed dietary intake once at } \\
\quad \text { baseline; did not account for possible } \\
\quad \text { changes in dietary intake over f/u } \\
\text { - Did not account for missing data } \\
\text { - No preregistered statistical plan; potential } \\
\text { for selective outcome reporting } \\
\text { Funding Sources: World Cancer Research } \\
\text { Fund International } \\
\text { Summary: aMED and mMED scores, with and } \\
\text { without alcohol, was not significantly } \\
\text { associated with risk of breast cancer afgter } \\
20.3 y \text { f flu. Results were also NS when } \\
\text { stratified by years of f/u, age at baseline, } \\
\text { smoking status, alcohol intake, BMl, physical }\end{array}$ \\
\hline
\end{tabular}

xxvi Fung TT, McCullough ML, Newby PK, et al.Diet-quality scores and plasma concentrations of markers of inflammation and endothelial dysfunction. Am J Clin Nutr 2005;82:163-73.

xxvii Trichopoulou A, Orfanos P, Norat T, et al. Modified Mediterranean diet and survival: EPIC-elderly prospective cohort study. BMJ 2005;330:991.

xxviii World Cancer Research Fund/American Institute for Cancer Research. Food, nutrition, physical activity, and the prevention of cancer: a global perspective. Washington, DC: AICR, 2007. 


\section{Study and \\ Participant \\ Characteristics}

Results
Confounding, Study Limitations, and Summary of Findings
Outcome assessment methods: Netherlands

Cancer Registry and the nationwide Dutch

Pathology Registry (Note: This paper also

examined various molecular subtypes of breast cancer; results for molecular subtypes were not extracted in this table)

\section{Voortman, 201724}

PCS (Rotterdam

Study)

The Netherlands

Analytic N: 9627

Subjects were $100 \%$ female, 64.1yo,

$26.3 \mathrm{~kg} / \mathrm{m} 2,32 \%$ never alcohol

\section{Excluded those}

without reliable dietary data, prevalent cancer cases, missing

outcome data $(\sim 19 \%$

of original sample) smokers, $61 \%<10 \mathrm{~g} / \mathrm{d}$

\section{Dietary patterns:}

- Dutch Dietary Guidelines 2015 score, continuous

- Positive components: Vegetables, legumes, fruit, nuts, whole grains, fish, dairy products, unsaturated fats and oils, tea

- Negative components: Replace refined grains with whole-grain products, red meat, processed meat, alcohol, sodium

Dietary assessment methods: 170 to $389-$ item, validated FFQ at baseline, $\sim 64 \mathrm{y}$

\section{Outcome assessment methods: nationwide} registry of histopathology and cytopathology, f/u with general practitioners

\section{Significant: N/A}

\section{Non-Significant:}

Dutch Dietary Guidelines 2015 score was not significantly associated with breast cancer $(n=273)$ after $10.9 \mathrm{y} / \mathrm{u}$. not significantly associated with breast cancer risk.

\section{Key confounders accounted for:}

Sex, age, race/ethnicity, education, employment status, alcohol intake, physical activity, smoking, BMI

Other: Cohort, total energy intake Limitations:

- Did not account for race/ethnicity, family history of the cancer outcome, hormonal contraceptive, menopausal status

- Only assessed dietary intake once at baseline; did not account for possible changes in dietary intake over $\mathrm{f} / \mathrm{u}$

- Did not account for missing data

- No preregistered statistical plan; potential for selective outcome reporting

Funding Sources: Erasmus University Medical Center; Erasmus University Rotterdam; Netherlands Organization for Health Research and Development; Research Institute for Diseases in the Elderly

Netherlands Genomics Initiative; Ministry of Education, Culture and Science; Ministry of Health, Welfare and Sports; the European Commission; Municipality of Rotterdam 
Confounding, Study Limitations, and Summary of Findings

Summary: Dutch Dietary Guidelines 2015 score at $64 y$ was not significantly associated with breast cancer after $11 y \mathrm{f} / \mathrm{u}$. 
Table 2. Summary of the results from studies that examined the relationship between dietary patterns and breast cancer ${ }^{\mathrm{xxix}}$

\begin{tabular}{|c|c|c|}
\hline $\begin{array}{c}\text { Article, Study } \\
\text { Design, Country }\end{array}$ & Significant Results & Non-Significant Results \\
\hline \multicolumn{3}{|l|}{$\begin{array}{l}\text { Randomized } \\
\text { Controlled Trials }\end{array}$} \\
\hline $\begin{array}{l}\text { Prentice, 201919 } \\
\text { RCT (Women's } \\
\text { Health Initiative } \\
\text { Dietary } \\
\text { Modification (DM) } \\
\text { trial) } \\
\text { United States } \\
\text { Analytic N: } 48835 \\
\text { (Intervention: } \\
\text { 19541, } \\
\text { Comparison: } \\
\text { 29294) }\end{array}$ & $\begin{array}{l}\text { Intervention group: Reduction in fat from } \\
\sim 35 \% \mathrm{E} \text { to } 20 \% \mathrm{E} \text { of energy, } 5 \text { servings/d } \\
\text { fruits and vegetables, } 6 \text { serving/d grains; } \\
\text { achieved reductions } 8-10 \% \mathrm{E} \text { of total, } \\
\text { saturated, and unsaturated fat, reductions } \\
\text { in } 8-10 \% \text { of carbohydrate, and increased } \\
\text { vegetables, fruit, and grains vs. } \\
\text { comparison. } \\
\text { Comparison group: Received written } \\
\text { health-related materials only; lower intakes } \\
\text { of fruits, vegetables, whole grains, and } \\
\text { total grains }\end{array}$ & $\begin{array}{l}\text { There were no significantly differences } \\
\text { in invasive postmenopausal breast } \\
\text { cancer risk during the intervention or } \\
\text { after } 19.6 y \text { f/u between the intervention } \\
\text { vs. comparison groups. }\end{array}$ \\
\hline
\end{tabular}

${ }^{x x i x}$ Abbreviations: AHEI-2010, Alternative Healthy Eating Index-2010; aMED, Alternative Mediterranean Diet Score ; BMI, body mass index; CSDLH, Canadian Study of Diet, Lifestyle and Health; CVD, Cardiovascular disease; d, day; DASH, Dietary Approaches to Stop Hypertension; DM, dietary modification; DP, Dietary pattern; \%E, \% of energy; EPIC, European Prospective Investigation into Cancer and Nutrition; ERDP, Estrogen-related dietary pattern, EVOO, extra-virgin olive oil; FFQ, food frequency questionnaire; FSAm-NPS, Nutrient Profiling System of the British Food Standards Agency; f/u, follow-up; HNFI, Healthy Nordic food index; HR, hazard ratio; IMI, Italian Mediterranean Index; MEDI-LITE, Mediterranean diet score; MD, Mediterranean diet; MDS, Mediterranean Diet Score; mMED, modified Mediterranean diet score; mo, month(s); MUFA, monounsaturated fat/fatty acids; N/A, Not applicable; NBSS, National Breast Screening Study; NIH, National Institutes of Health; NOVA, Ultra-processed food score; NS, Not significant; NR, Not reported; PCS, prospective cohort study; pt, point; RCT, randomized controlled trial; SEER, Surveillance, Epidemiology, and End Results Program; SFA, saturated fat/fatty acids; WCRF/AICR, World Cancer Research Fund/American Institute for Cancer Research; wk, weeks; y, years 


\begin{tabular}{|c|c|c|c|}
\hline $\begin{array}{c}\text { Article, Study } \\
\text { Design, Country }\end{array}$ & Dietary Patterns & Significant Results & Non-Significant Results \\
\hline $\begin{array}{l}\text { Thomson, 2014² } \\
\text { RCT (Women's } \\
\text { Health Initiative } \\
\text { Dietary } \\
\text { Modification (DM) } \\
\text { trial) } \\
\text { United States } \\
\text { Analytic N: } 48835 \\
\text { (Intervention: } \\
\text { 19541, } \\
\text { Comparison: } \\
\text { 29294) }\end{array}$ & $\begin{array}{l}\text { Intervention group: Reduction in fat from } \\
\sim 35 \% \mathrm{E} \text { to } 20 \% \mathrm{E}, 5 \text { servings/d fruits and } \\
\text { vegetables, } 6 \text { serving/d grains; achieved } \\
\text { reductions } 8-10 \% \mathrm{E} \text { of total, saturated, and } \\
\text { unsaturated fat, reductions in } 8-10 \% \text { of } \\
\text { carbohydrate, and increased vegetables, } \\
\text { fruit, and grains vs. comparison. } \\
\text { Comparison group: Received written } \\
\text { health-related materials only; lower intakes } \\
\text { of fruits, vegetables, whole grains, and } \\
\text { total grains }\end{array}$ & $\begin{array}{l}\text { Women with higher baseline fat intake (quartiles) had } \\
\text { significantly reduced risk of postmenopausal breast } \\
\text { cancer ( } p=0.03 \text { ): } \\
\quad \text { During the intervention: HR: } 0.76,95 \% \mathrm{Cl} \text { : } \\
\quad 0.62,0.92 \\
\text { During post-intervention f/u: HR: } 1.11,95 \% \mathrm{Cl}: 0.84 \text {, } \\
1.4\end{array}$ & $\begin{array}{l}\text { There were no significantly differences } \\
\text { in invasive postmenopausal breast } \\
\text { cancer risk after } 5.2 y, 8.5 y \text {, or } 13.5 y \mathrm{f} / \mathrm{u} \\
\text { between the intervention vs. } \\
\text { comparison groups. }\end{array}$ \\
\hline
\end{tabular}




\begin{tabular}{|c|c|c|c|}
\hline $\begin{array}{c}\text { Article, Study } \\
\text { Design, Country }\end{array}$ & Dietary Patterns & Significant Results & Non-Significant Results \\
\hline $\begin{array}{l}\text { Toledo, 2015³ } \\
\text { RCT (PREDIMED } \\
\text { Trial) } \\
\text { Spain } \\
\text { Analytic N: } 4152\end{array}$ & $\begin{array}{l}\text { Mediterranean diet (MD) with extra-virgin } \\
\text { olive oil (EVOO): MD supplemented with } \\
\text { EVOO (provided } 1 \mathrm{~L} \text { per week); MD diet } \\
\text { included live oil for cooking and dressing; } \\
\text { fruit, vegetables, legumes and fish; } \\
\text { reduced total meat consumption, white } \\
\text { meat instead of red or processed meat; } \\
\text { homemade sauce with tomato, garlic, } \\
\text { onion and spices with olive oil to dress } \\
\text { vegetables, pasta, rice and other dishes; } \\
\text { avoidance of butter, cream, fast food, } \\
\text { sweets, pastries and sugar-sweetened } \\
\text { beverages; and moderate red wine } \\
\text { MD with mixed nuts: MD supplemented } \\
\text { with mixed nuts (MD-nuts), with } 30 \mathrm{~g} \text { per } \\
\text { day of mixed nuts (15g walnuts, } 7.5 \mathrm{~g} \\
\text { hazelnuts and } 7.5 \mathrm{~g} \text { almonds) } \\
\text { Control diet: Consumed similar food } \\
\text { groups, but were counseled to also } \\
\text { decrease fat intake in accordance with } \\
\text { American Heart Association guidelines }\end{array}$ & 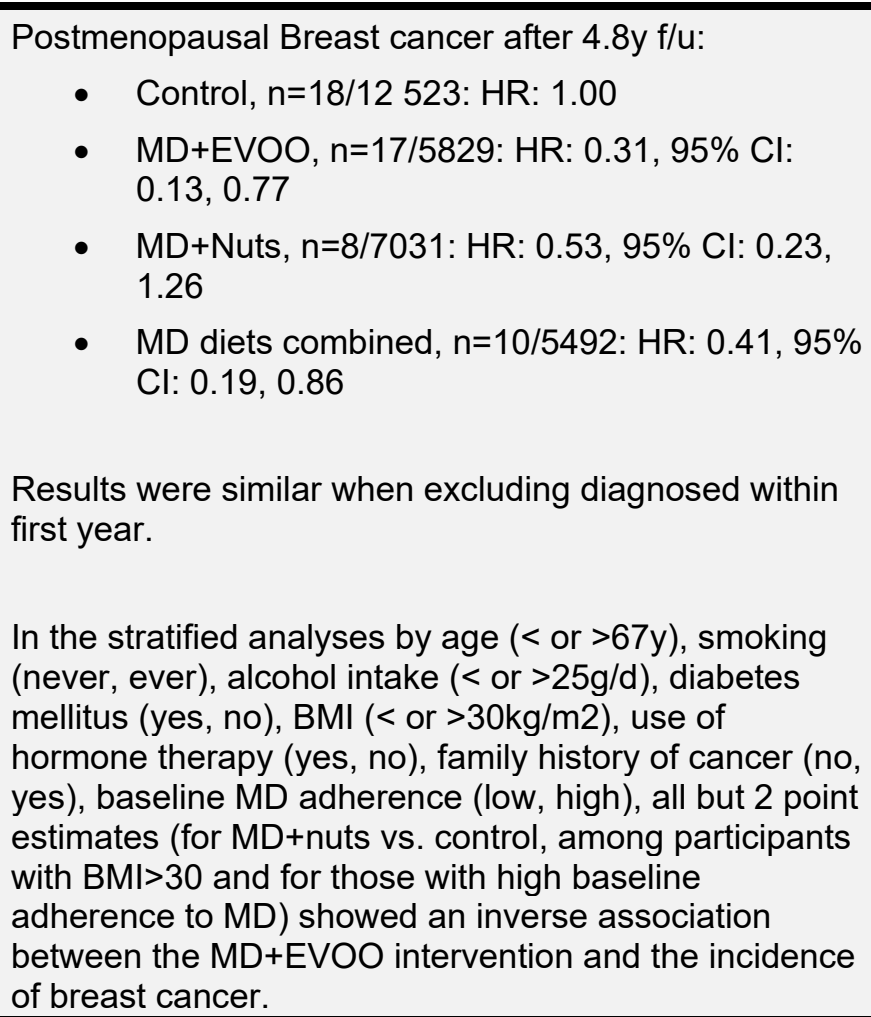 & \\
\hline
\end{tabular}




\begin{tabular}{|c|c|c|c|}
\hline $\begin{array}{c}\text { Article, Study } \\
\text { Design, Country }\end{array}$ & Dietary Patterns & Significant Results & Non-Significant Results \\
\hline $\begin{array}{l}\text { Catsburg, } 2015^{25} \\
\text { Nested Case- } \\
\text { Control Study and } \\
\text { PCS (Canadian } \\
\text { Study of Diet, } \\
\text { Lifestyle and } \\
\text { Health (CSDLH), } \\
\text { National Breast } \\
\text { Screening Study } \\
\text { (NBSS)) } \\
\text { Canada }\end{array}$ & $\begin{array}{l}\text { "Healthy" pattern (PCA): Higher loadings for } \\
\text { vegetable and legume food groups }\end{array}$ & $\begin{array}{l}\text { "Healthy" pattern at } 60 \mathrm{y} \text { (CSLDH) and total breast } \\
\text { cancer after 13y f/u: } \\
\text { - Q1, n=125: HR: } 1.00 \\
\text { - Q2, n=258: HR: } 0.93,95 \% \mathrm{Cl}: 0.74,1.18 \\
\text { - Q4, n=270: HR: } 0.78,95 \% \mathrm{Cl}: 0.61,0.99 \\
\text { - } \mathrm{Q} 5, \mathrm{n}=452: \mathrm{HR}: 0.80,95 \% \mathrm{Cl}: 0.64,1.01 \\
\text { - } 0.73,95 \% \mathrm{Cl}: 0.58,0.91 \\
\text { Results for the }=0.0001 \\
\text { significant when pre- and postmenopausal breast } \\
\text { cancer were analyzed separately. }\end{array}$ & $\begin{array}{l}\text { "Healthy" pattern at } 40-59 y \text { (NBSS) } \\
\text { was not significantly associated with } \\
\text { breast cancer after } \sim 23 y \text { f/u, in all } \\
\text { women combined and when pre- and } \\
\text { postmenopausal women were } \\
\text { analyzed separately. }\end{array}$ \\
\hline $\begin{array}{l}\text { Analytic N: } \\
\text { CSDLH: } 4417 \\
\text { NBSS: } 49410\end{array}$ & $\begin{array}{l}\text { "Ethnic" pattern (PCA): Higher loadings for } \\
\text { rice, spinach, fish, tofu, liver, eggs, and salted } \\
\text { and dried meat }\end{array}$ & & $\begin{array}{l}\text { "Ethnic" pattern at } 40-59 y \text { (NBSS) was } \\
\text { not significantly associated with breast } \\
\text { cancer after } \sim 23 y \text { f/u, in all women } \\
\text { combined and when pre- and } \\
\text { postmenopausal women were } \\
\text { analyzed separately. } \\
\text { "Ethnic" pattern at } \sim 60 y(C S L D H) \text { was } \\
\text { not significantly associated with breast } \\
\text { cancer after } 13 y \text { f } / \mathrm{u}(p=0.073 \text { ), in all } \\
\text { women combined and when pre- and } \\
\text { postmenopausal women were } \\
\text { analyzed separately. }\end{array}$ \\
\hline
\end{tabular}




\begin{tabular}{|c|c|c|c|}
\hline $\begin{array}{l}\text { Article, Study } \\
\text { Design, Country }\end{array}$ & Dietary Patterns & Significant Results & Non-Significant Results \\
\hline & $\begin{array}{l}\text { "Meat and potatoes" pattern: Higher loadings } \\
\text { for red meat groups and potatoes }\end{array}$ & 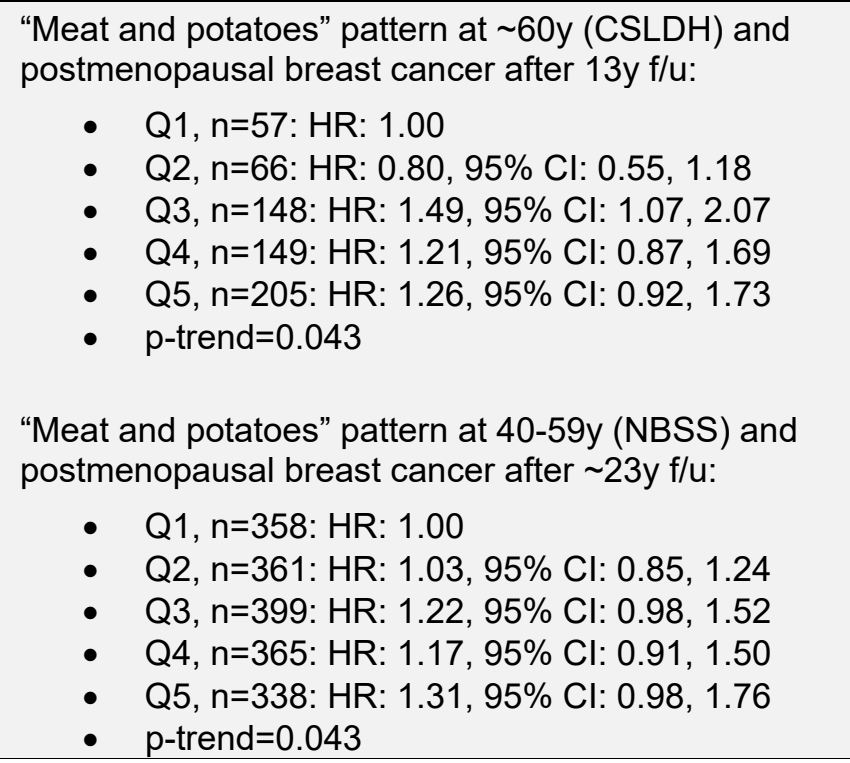 & $\begin{array}{l}\text { "Meat and potatoes" pattern at } 40-59 y \\
\text { (NBSS) was not significantly } \\
\text { associated with breast cancer after } \\
\sim 23 y \text { f/u, in all women combined or in } \\
\text { premenopausal women. } \\
\text { "Meat and potatoes" pattern at } \sim 60 y \\
\text { (CSLDH) was not significantly } \\
\text { associated with breast cancer after 13y } \\
\text { f/u, in all women combined or in } \\
\text { premenopausal women. }\end{array}$ \\
\hline $\begin{array}{l}\text { Deschasaux, } \\
\text { 2018 }^{4} \\
\text { PCS (EPIC) } \\
\text { Denmark, France, } \\
\text { Germany, Greece, } \\
\text { Italy, the } \\
\text { Netherlands, } \\
\text { Norway, Spain, } \\
\text { Sweden, UK } \\
\text { Analytic N: } 471495\end{array}$ & $\begin{array}{l}\text { Nutrient Profiling System of the British Food } \\
\text { Standards Agency (modified version) (FSAm- } \\
\text { NPS) score } \\
\text { - Overall diet score assigned based on } \\
\text { energy, sugar, saturated fatty acid, sodium, } \\
\text { fibers, proteins, and } \\
\text { fruits/vegetables/legumes/nuts. } \\
\text { - Higher FSAm-NPS score had higher } \\
\text { intakes of alcohol, energy and red and } \\
\text { processed meat, lower intakes of dietary } \\
\text { fibers, vegetables, fruit, fish, and lean } \\
\text { meat }\end{array}$ & $\begin{array}{l}\text { FSAm-NPS score at 51y and postmenopausal breast } \\
\text { cancer after 15.3y f/u: } \\
\text { - Q1, n=2093: HR: } 1.00 \\
\text { - Q2, n=2303: HR: } 1.04,95 \% \mathrm{Cl}: 0.98,1.1 \\
\text { - Q3, n=2403: HR: } 1.03,95 \% \mathrm{Cl}: 0.97,1.10 \\
\text { - Q4, } \mathrm{n}=2682: \mathrm{HR}: 1.07,95 \% \mathrm{Cl}: 1.01,1.14 \\
\text { - Q5, } \mathrm{n}=2636: \mathrm{HR}: 1.06,95 \% \mathrm{Cl}: 0.99,1.14 \\
\text { - p-trend=0.05 } \\
\text { Continuous, per } 2 \mathrm{pt} \text { increment, } \mathrm{n}=12063: \mathrm{HR}: 1.02 \text {, } \\
\text { 95\% Cl: } 1.00,1.04 ; \text {-trend }=0.05\end{array}$ & \\
\hline
\end{tabular}




\begin{tabular}{|c|c|c|c|}
\hline $\begin{array}{c}\text { Article, Study } \\
\text { Design, Country }\end{array}$ & Dietary Patterns & Significant Results & Non-Significant Results \\
\hline $\begin{array}{l}\text { Deschasaux, } \\
2017^{5} \\
\text { PCS (NutriNet- } \\
\text { Santé) } \\
\text { France } \\
\text { Analytic N: } 46864\end{array}$ & $\begin{array}{l}\text { Nutrient Profiling System of the British Food } \\
\text { Standards Agency (modified version) (FSAm- } \\
\text { NPS) score } \\
\text { - Overall diet score assigned based on } \\
\text { energy, sugar, saturated fatty acid, sodium, } \\
\text { fibers, proteins, and } \\
\text { fruits/vegetables/legumes/nuts. } \\
\text { Higher FSAm-NPS score had higher } \\
\text { intakes of protein, fiber, fruit, vegetables, } \\
\text { legumes, poultry, fish, and dairy, and } \\
\text { lower intakes of energy, alcohol, fat, } \\
\text { carbohydrate, red meat, processed meat, } \\
\text { lower intakes of dietary fibers, vegetables, } \\
\text { fruit, fish, and lean meat }\end{array}$ & 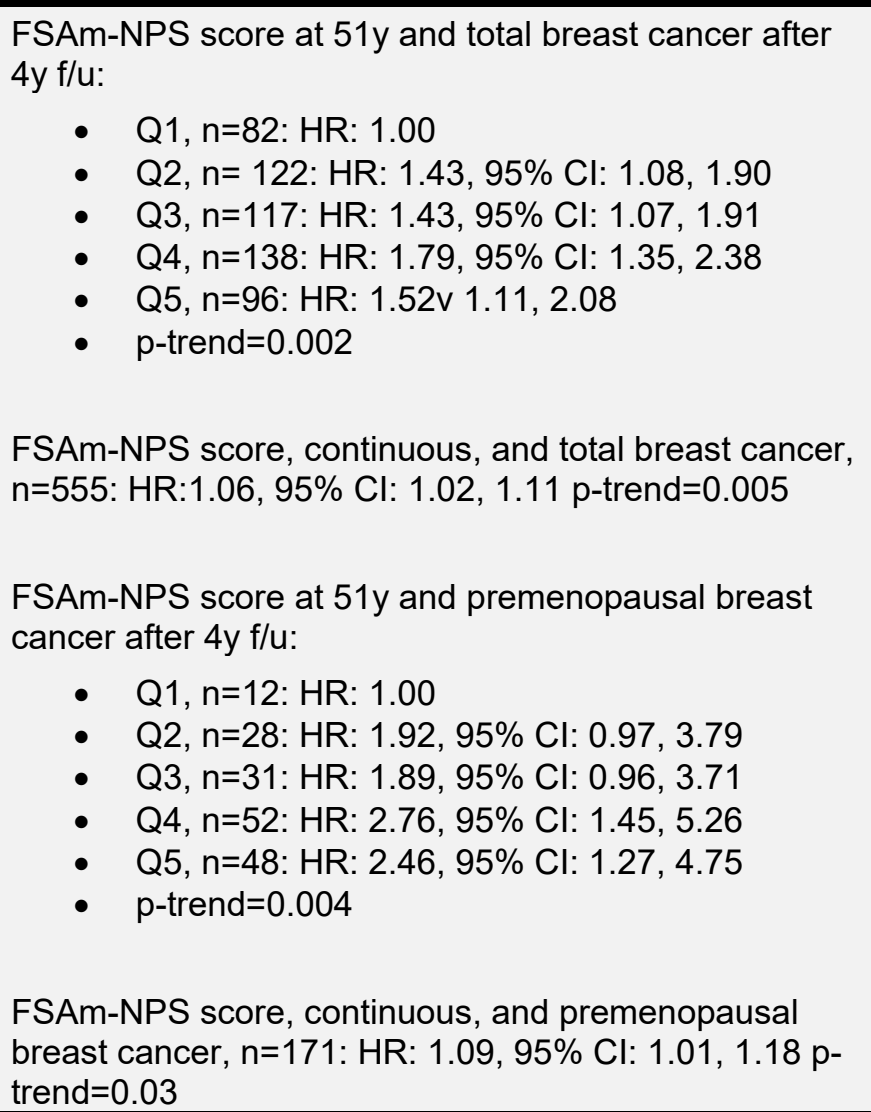 & $\begin{array}{l}\text { FSAm-NPS score at 51y (categorical } \\
\text { and continuous) was not significantly } \\
\text { associated ( } p=0.09, p=0.06 \text { ) with } \\
\text { postmenopausal breast cancer after } 4 y \\
\text { f/u: }\end{array}$ \\
\hline
\end{tabular}




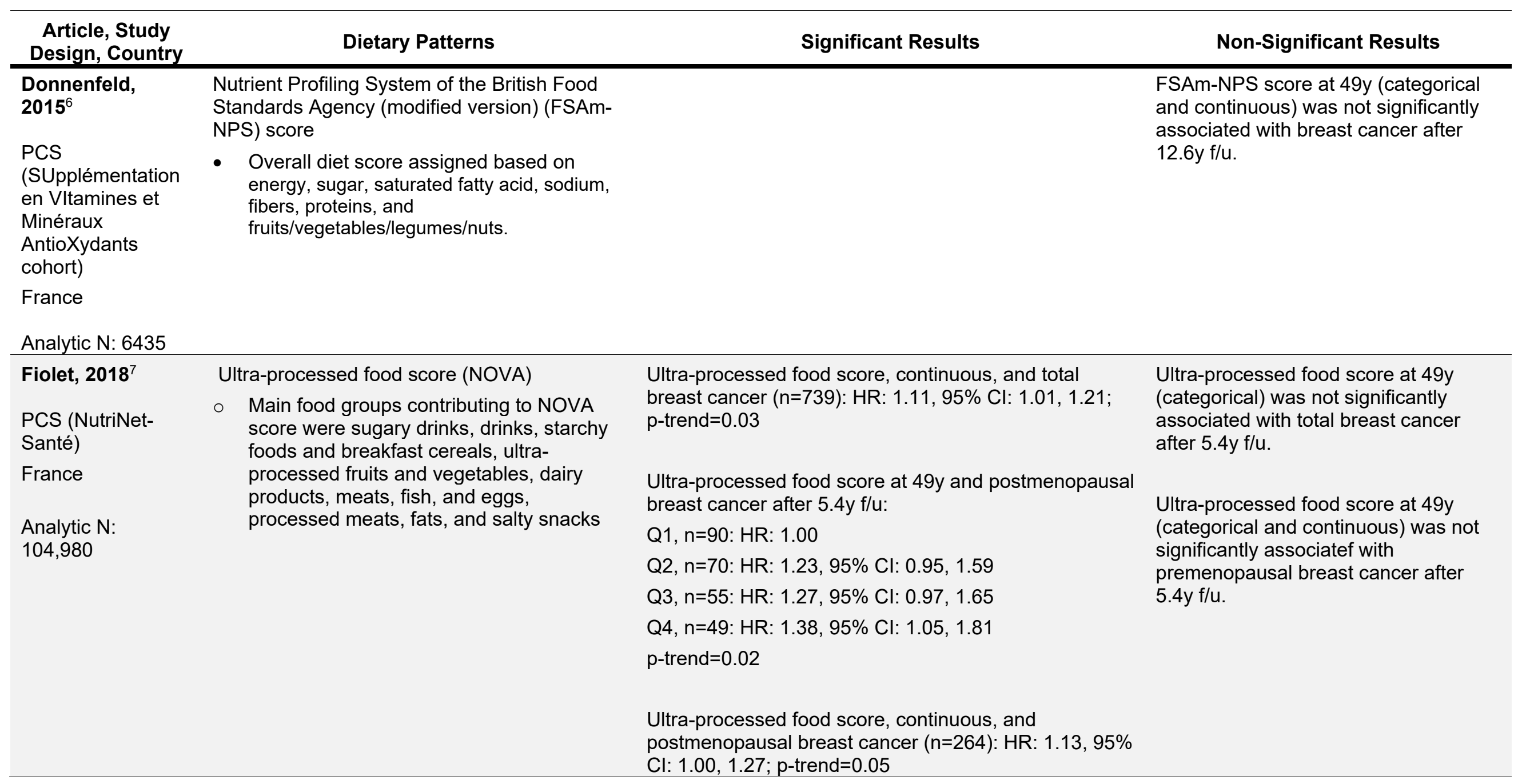




\begin{tabular}{|c|c|c|c|}
\hline $\begin{array}{c}\text { Article, Study } \\
\text { Design, Country }\end{array}$ & Dietary Patterns & Significant Results & Non-Significant Results \\
\hline $\begin{array}{l}\text { Guinter, 2018a } \\
\text { (IJC) }^{8} \\
\text { PCS (Prostate, } \\
\text { Lung, Colorectal } \\
\text { and Ovarian } \\
\text { Screening Trial) } \\
\text { United States } \\
\text { Analytic N: } 27488\end{array}$ & $\begin{array}{l}\text { Estrogen-related dietary pattern (ERDP; RRR, } \\
\text { response variables: unconjugated estradiol, } \\
\text { ratio of 2- and 16-hydroxylated estrogen } \\
\text { metabolites): } \\
\text { - Higher loading for non-whole/refined } \\
\text { grains, tomatoes, cruciferous vegetables, } \\
\text { cheese, high omega-3 fish/shellfish, } \\
\text { franks/luncheon meats } \\
\text { - Lower loadings for nuts/seeds, other } \\
\text { vegetables, low omega-3 fish/shellfish, } \\
\text { yogurt, coffee }\end{array}$ & 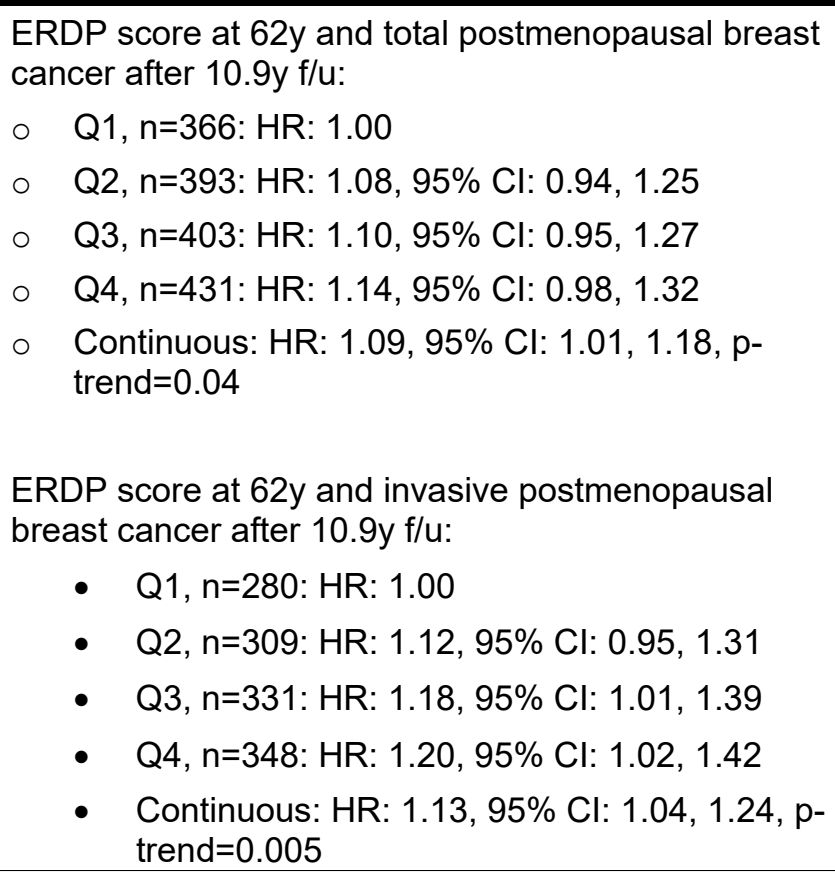 & \\
\hline $\begin{array}{l}\text { Guinter, 2018b } \\
\text { (CEBP) } \\
\text { PCS (Sister Study) }\end{array}$ & $\begin{array}{l}\text { Estrogen-related dietary pattern (ERDP; RRR, } \\
\text { response variables: unconjugated estradiol, } \\
\text { ratio of } 2-\text { and 16-hydroxylated estrogen } \\
\text { metabolites): }\end{array}$ & & $\begin{array}{l}\text { ERDP score at } 58 \mathrm{y} \text { was not } \\
\text { significantly associated with total } \\
\text { postmenopausal breast cancer after 6- } \\
12 \mathrm{y} \text { f/u. }\end{array}$ \\
\hline $\begin{array}{l}\text { United States } \\
\text { Analytic N: } 37925\end{array}$ & $\begin{array}{l}\text { Higher loading for non-whole/refined } \\
\text { grains, tomatoes, cruciferous vegetables, } \\
\text { cheese, high omega-3 fish/shellfish, } \\
\text { franks/luncheon meats } \\
\text { Lower loadings for nuts/seeds, other } \\
\text { vegetables, low omega-3 fish/shellfish, } \\
\text { yogurt, coffee }\end{array}$ & & $\begin{array}{l}\text { ERDP score at } 58 \mathrm{y} \text { was not } \\
\text { significantly associated with invasive } \\
\text { postmenopausal breast cancer after } 6- \\
12 \mathrm{y} \text { f/u. }\end{array}$ \\
\hline
\end{tabular}




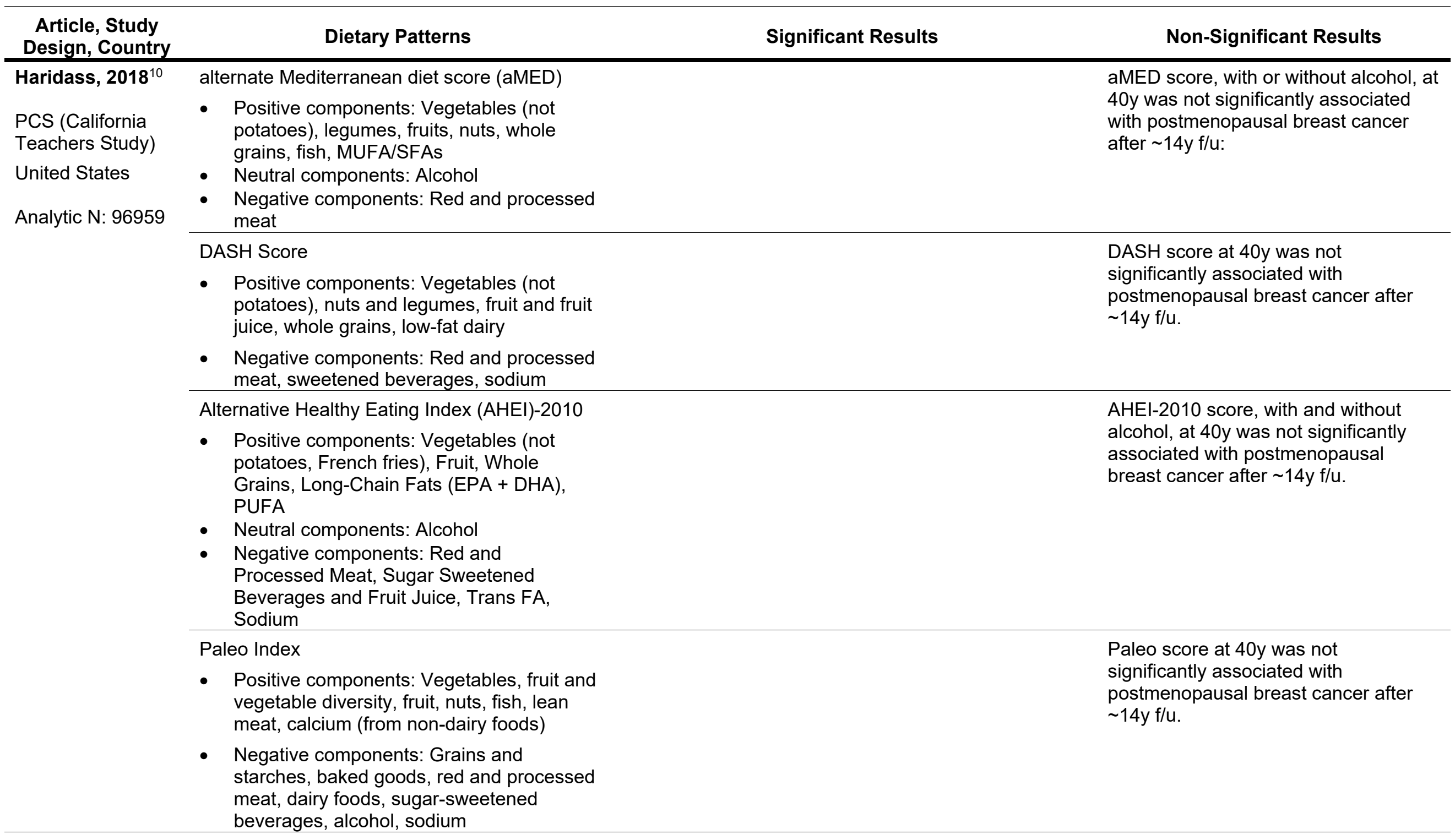




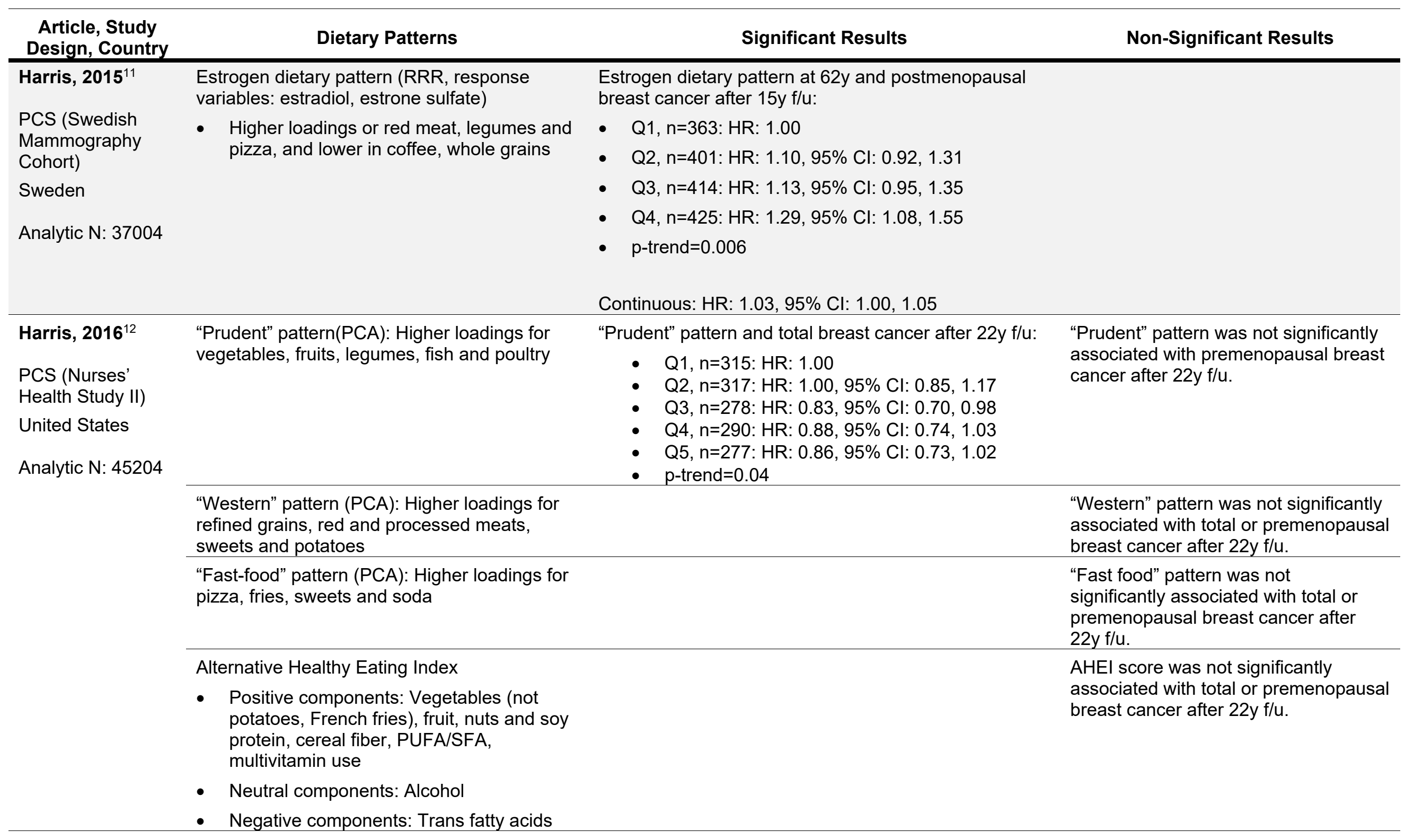




\begin{tabular}{|c|c|c|c|}
\hline $\begin{array}{c}\text { Article, Study } \\
\text { Design, Country }\end{array}$ & Dietary Patterns & Significant Results & Non-Significant Results \\
\hline $\begin{array}{l}\text { Harris, } 2017^{13} \\
\text { PCS (Nurses' } \\
\text { Health Study II) } \\
\text { United States } \\
\text { Analytic N: } 45204\end{array}$ & $\begin{array}{l}\text { Adherence to a dietary pattern identified via } \\
\text { reduced rank regression (response variables: } \\
\text { C-reactive protein, IL6, and TNFa receptor 2), } \\
\text { categorical (quintiles): } \\
\text { - "Inflammatory" dietary pattern: Higher } \\
\text { loadings for sugar-sweetened and diet soft } \\
\text { drinks, refined grains, red and processed } \\
\text { meat, margarine, corn, other vegetables, } \\
\text { and fish, and lower in green leafy } \\
\text { vegetables, yellow vegetables, cruciferous } \\
\text { vegetables, and coffee }\end{array}$ & 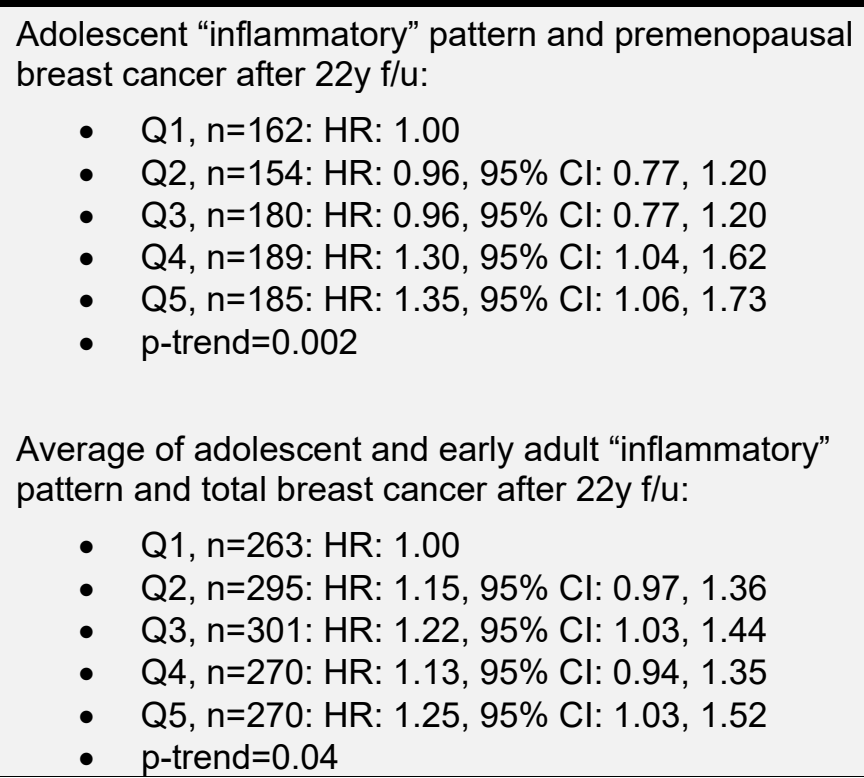 & $\begin{array}{l}\text { Adolescent "inflammatory" pattern was } \\
\text { not significantly associated with total or } \\
\text { postmenopausal breast cancer after } \\
\text { 22y f/u. } \\
\text { Results were similar when the early } \\
\text { adulthood "inflammatory" pattern was } \\
\text { analyzed, and when adolescent and } \\
\text { early adult "inflammatory" patterns } \\
\text { were averaged, except for the results } \\
\text { for all cases of breast cancer. }\end{array}$ \\
\hline $\begin{array}{l}\text { Kane-Diallo, } \\
\mathbf{2 0 1 8}^{14} \\
\text { PCS (NutriNet- } \\
\text { Sante study) } \\
\text { France } \\
\text { Analytic N: } 42544\end{array}$ & $\begin{array}{l}\text { "Pro plant-based" dietary score } \\
\text { Higher in plant foods: vegetables, } \\
\text { legumes, fruits, cereal products, potatoes, } \\
\text { nuts, vegetables oils } \\
\text { - Lower in animal foods: red and processed } \\
\text { meat, eggs, animal fat, dairy products, } \\
\text { seafood }\end{array}$ & & $\begin{array}{l}\text { "Pro plant-based" dietary score at } 49 y \\
\text { was not significantly associated with } \\
\text { risk of total, premenopausal, or } \\
\text { postmenopausal breast cancer after } \\
4.3 y \text { f/u. }\end{array}$ \\
\hline Kojima, $2017^{15}$ & $\begin{array}{l}\text { "Vegetable" pattern (factor analysis): Higher } \\
\text { loadings for vegetables, potatoes, seaweed, } \\
\text { tofu, fruits, fresh fish, eggs, and miso soup }\end{array}$ & & $\begin{array}{l}\text { "Vegetable pattern" at } 55 \mathrm{y} \text { was not } \\
\text { significantly associated with } \\
\text { premenopausal or postmenopausal } \\
\text { breast cancer after } 16.9 \mathrm{y} / \mathrm{u} \text {. }\end{array}$ \\
\hline
\end{tabular}




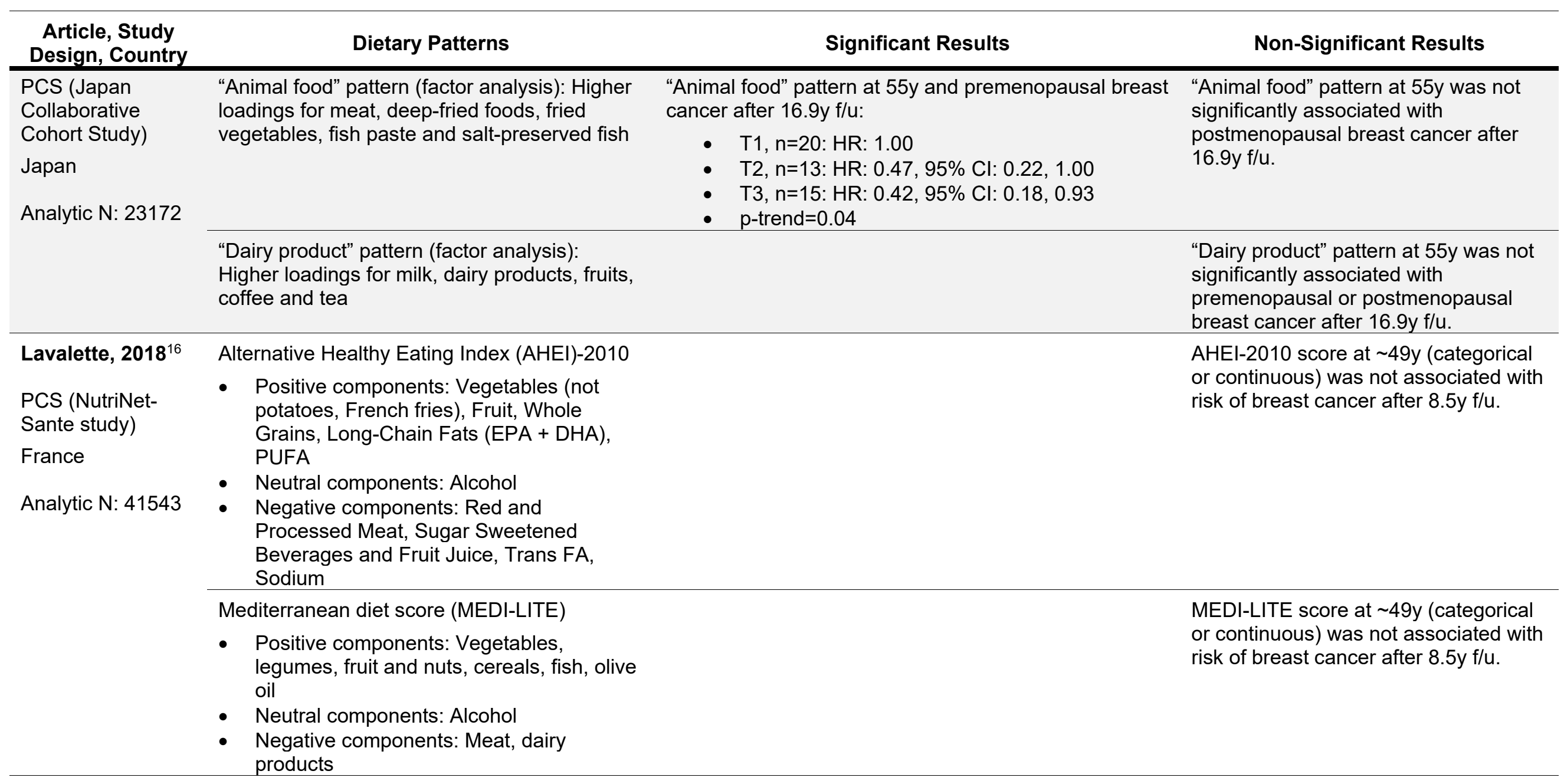




\begin{tabular}{|c|c|c|c|}
\hline $\begin{array}{c}\text { Article, Study } \\
\text { Design, Country }\end{array}$ & Dietary Patterns & Significant Results & Non-Significant Results \\
\hline & $\begin{array}{l}\text { French National Nutrition Health Program- } \\
\text { Guideline Score (PNNS-GS) } \\
\text { - Positive components: Vegetables and } \\
\text { Fruit, Seafood, Vegetable Fat } \\
\text { - Neutral components: Breads, cereals, } \\
\text { potatoes, legumes, meat and poultry, } \\
\text { seafood, and eggs, milk and dairy } \\
\text { products, alcohol } \\
\text { Negative components: Sweetened foods, } \\
\text { soda, added fat, salt }\end{array}$ & & $\begin{array}{l}\text { PNNS-GS score at } \sim 49 \mathrm{y} \text { (categorical } \\
\text { or continuous) was not associated with } \\
\text { risk of breast cancer after } 8.5 \mathrm{y} / \mathrm{u} \text {. }\end{array}$ \\
\hline $\begin{array}{l}\text { Li, } 2015^{17} \\
\text { PCS (Swedish } \\
\text { Women's Lifestyle } \\
\text { and Health cohort) } \\
\text { Sweden } \\
\text { Analytic N: } 44296\end{array}$ & $\begin{array}{l}\text { Healthy Nordic food index (HNFI) } \\
\text { - Positive components: Cabbage, root } \\
\text { vegetables, apples and pears, rye bread, } \\
\text { oatmeal, fish }\end{array}$ & & $\begin{array}{l}\text { All women, } \mathrm{HNFI} \text { at } 39 \mathrm{y} \text { was not } \\
\text { significantly associated with total, } \\
\text { premenopausal, or postmenopausal } \\
\text { breast cancer after } 20 \mathrm{y} / \mathrm{u} \text {. }\end{array}$ \\
\hline $\begin{array}{l}\text { McKenzie, } 2015^{18} \\
\text { PCS (EPIC) } \\
\text { Denmark, France, } \\
\text { Germany, Greece, } \\
\text { Italy, the } \\
\text { Netherlands, } \\
\text { Norway, Spain, } \\
\text { Sweden and the } \\
\text { United Kingdom } \\
\text { Analytic N: } 242918\end{array}$ & $\begin{array}{l}\text { Diet score } \\
\text { - Positive components: Cereal fiber, folate, } \\
\text { PUFA/SFA, fatty fish, fruits and vegetables } \\
\text { - Negative components: margarine, } \\
\text { glycemic load }\end{array}$ & 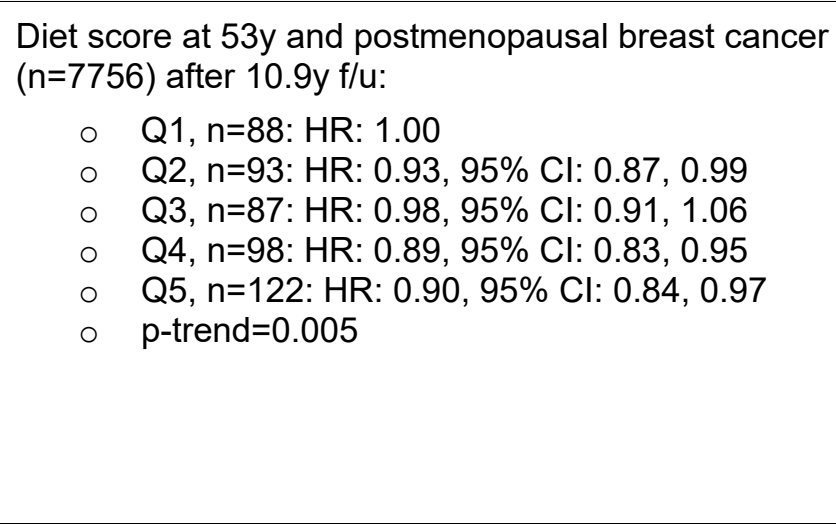 & \\
\hline
\end{tabular}




\begin{tabular}{|c|c|c|c|}
\hline $\begin{array}{c}\text { Article, Study } \\
\text { Design, Country }\end{array}$ & Dietary Patterns & Significant Results & Non-Significant Results \\
\hline $\begin{array}{l}\text { Nomura, 2016 } \\
\text { PCS (Black } \\
\text { Women's Health } \\
\text { Study) } \\
\text { United States } \\
\text { Analytic N: } 49103\end{array}$ & $\begin{array}{l}\text { WCRF/AICR score, diet only } \\
\text { - Positive components: Vegetables and fruit, } \\
\text { dietary fiber } \\
\text { - Negative components: Red and processed } \\
\text { meat, sugary drinks, alcohol, sodium, } \\
\text { energy-dense foods }\end{array}$ & $\begin{array}{l}\text { WCRF/AICR score, diet only, time-varying, continuous } \\
\text { (per } 0.5 \text { unit increase), } n=1766: \mathrm{HR}: 0.91,95 \% \mathrm{Cl}: 0.83 \text {, } \\
0.99 ; p \text {-trend=0.04 } \\
\text { When results were analyzed by menopausal status, } \\
\text { results were borderline significant in premenopausal } \\
\text { breast cancer ( } p=0.06) \text {, and non-significant for } \\
\text { postmenopausal breast cancer. }\end{array}$ & $\begin{array}{l}\text { WCRF/AICR score, diet only, at } \\
\text { baseline (categorical, continuous, and } \\
\text { time-varying-categorical), 38yo, was } \\
\text { not significantly associated with breast } \\
\text { cancer after } 13.9 y \text { f/u. } \\
\text { Results were similar when pre- and } \\
\text { post-menopausal breast cancer were } \\
\text { analyzed separately. }\end{array}$ \\
\hline
\end{tabular}




\begin{tabular}{|c|c|c|c|}
\hline $\begin{array}{c}\text { Article, Study } \\
\text { Design, Country }\end{array}$ & Dietary Patterns & Significant Results & Non-Significant Results \\
\hline $\begin{array}{l}\text { Penniecook- } \\
\text { Sawyers, 201620 } \\
\text { PCS (Adventist } \\
\text { Health Study-2) } \\
\text { United States } \\
\text { Analytic N: } 50404\end{array}$ & $\begin{array}{l}\text { "Vegetarian" patterns: } \\
\text { - "Vegans"xxx: Red meat, poultry, fish; eggs; } \\
\text { and dairy <1 time/mo } \\
\text { "Lacto-ovo-vegetarian": Red meat, poultry, } \\
\text { and fish <1 time/mo, eggs or dairy }>1 \\
\text { time/mo } \\
\text { "Pesco-vegetarian": Red meat or poultry } \\
<1 \text { time/mo, fish }>1 \text { time/mo, and } \\
\text { eggs/dairy in any amount } \\
\text { "Semi-vegetarian": Red meat or poultry }>1 \\
\text { time/mo, and all meats combined } \\
\text { (including fish) <1 time/wk and eggs/dairy } \\
\text { in any amount } \\
\text { "Non-vegetarians": Red meat and poultry } \\
>1 \text { time/mo and all meats combined } \\
\text { (including fish) }>1 \text { time/wk, and eggs/dairy } \\
\text { in any amount } \\
\text { "Vegetarians" vs. "nonvegetarians" consumed } \\
\text { higher amounts of fruits, vegetables, } \\
\text { avocados, non-fried potatoes, whole grains, } \\
\text { legumes, soya foods, nuts and seeds, and } \\
\text { was observed among vegetarians; and lower } \\
\text { amounts of meats, dairy products, eggs, } \\
\text { refined grains, added fats, sweets, snack } \\
\text { foods and non-water beverages }\end{array}$ & & $\begin{array}{l}\text { "Vegetarian" pattern at } 64 y \text { was not } \\
\text { significantly associated with total or } \\
\text { postmenopausal breast cancer after } \\
7.8 y \text { f.u. Results were similar when } \\
\text { analyzed by non-black or black race. }\end{array}$ \\
\hline
\end{tabular}




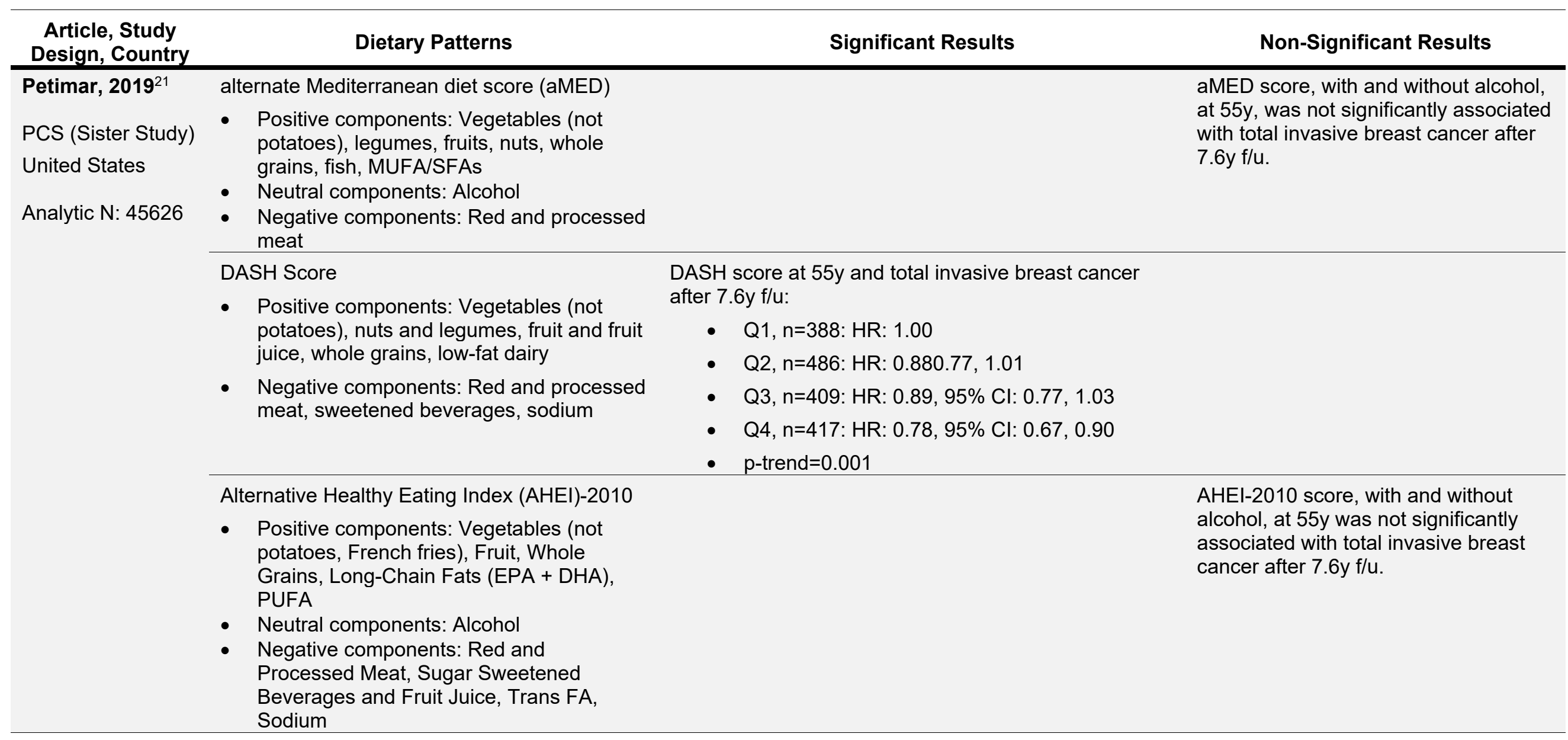




\begin{tabular}{|c|c|c|c|}
\hline $\begin{array}{c}\text { Article, Study } \\
\text { Design, Country }\end{array}$ & Dietary Patterns & Significant Results & Non-Significant Results \\
\hline $\begin{array}{l}\text { Pot, 2014 } \\
\text { Nested Case- } \\
\text { Control Study (UK } \\
\text { Dietary Cohort } \\
\text { Consortium) } \\
\text { United Kingdom } \\
\text { Analytic N: } 601\end{array}$ & $\begin{array}{l}\text { Mediterranean Diet Score (MDS), with and } \\
\text { without alcohol } \\
\text { - Positive components: Vegetables, } \\
\text { legumes, fruit and nuts, cereals, fish, } \\
\text { MUFA/SFA } \\
\text { - Neutral components: Alcohol } \\
\text { - Negative components: Red and processed } \\
\text { meat, dairy products }\end{array}$ & & $\begin{array}{l}\text { MDS score, with and without alcohol, } \\
\text { was not significantly associated with } \\
\text { total or postmenopausal breast cancer }\end{array}$ \\
\hline \multirow{3}{*}{$\begin{array}{l}\text { Analytic N: } 601 \\
\text { cases, } 1891 \\
\text { controls }\end{array}$} & $\begin{array}{l}\text { PCA pattern Higher loadings for cheese, } \\
\text { crisps and savory snacks, fresh fruit, legumes, } \\
\text { low fat milk, nuts and seeds, other fruit, } \\
\text { rice/pasta/other grains, sauces, vegetable } \\
\text { mixed dishes, and lower in potatoes, poultry, } \\
\text { and red meat }\end{array}$ & & $\begin{array}{l}\text { PCA dietary pattern score was not } \\
\text { significantly associated with total or } \\
\text { postmenopausal breast cancer. }\end{array}$ \\
\hline & $\begin{array}{l}\text { "High alcohol" (RRR, response variables: } \\
\text { alcohol, total fat, fiber): Higher loadings for } \\
\text { wines, spirits, and beers and ciders }\end{array}$ & $\begin{array}{l}\text { "High alcohol" pattern and breast cancer }(\mathrm{n}=387 \\
\text { cases): } \\
\text { - T1: HR: } 1.00 \\
\text { - T2: HR: } 1.04,95 \% \mathrm{Cl}: 0.77,1.39 \\
\text { - T3: HR: } 1.28,95 \% \mathrm{Cl}: 0.95,1.71 \\
\text { - } \quad \text {-trend }=0.08\end{array}$ & \\
\hline & & $\begin{array}{l}\text { "High alcohol" pattern and postmenopausal breast } \\
\text { cancer ( }=409 \text { cases): } \\
\text { - T1: HR: } 1.00 \\
\text { - T2: HR: } 1.14,95 \% \mathrm{Cl}: 0.84,1.55 \\
\text { - T3: HR: } 1.46,95 \% \mathrm{Cl}: 0.08,1.98 \\
\text { - } \quad \text {-trend }=0.01\end{array}$ & \\
\hline
\end{tabular}




\begin{tabular}{|c|c|c|c|}
\hline $\begin{array}{l}\text { Article, Study } \\
\text { Design, Country }\end{array}$ & Dietary Patterns & Significant Results & Non-Significant Results \\
\hline & $\begin{array}{l}\text { "High fiber" (RRR, response variables: alcohol, } \\
\text { total fat, fiber): Higher loadings for fiber, fresh } \\
\text { fruit, raw and boiled vegetables, high fiber } \\
\text { bread, high fiber breakfast cereals, lower in } \\
\text { alcohol and total fat }\end{array}$ & & $\begin{array}{l}\text { "High fiber" pattern score was not } \\
\text { significantly associated with total or } \\
\text { postmenopausal breast cancer. }\end{array}$ \\
\hline \multirow{5}{*}{$\begin{array}{l}\text { Shin, 2016 } \\
\text { 22 } \\
\text { PCS (Japan Public } \\
\text { Health Center- } \\
\text { based Prospective } \\
\text { Study (JPHC } \\
\text { Study)) } \\
\text { Japan } \\
\text { Analytic N: } 49552\end{array}$} & $\begin{array}{l}\text { Prudent pattern (PCA): Higher loadings for } \\
\text { vegetables, fruits, soya products, potatoes, } \\
\text { seaweed, mushroom, and fish }\end{array}$ & & $\begin{array}{l}\text { Prudent pattern score at } 57 y \text { was not } \\
\text { significantly associated with total, } \\
\text { premenopausal, or postmenopausal } \\
\text { breast cancer after } 14.6 \mathrm{y} / \mathrm{u} \text {. }\end{array}$ \\
\hline & \multirow[t]{3}{*}{$\begin{array}{l}\text { Westernized pattern (PCA): Higher loadings } \\
\text { for bread, meat, processed meats, dairy } \\
\text { products, soup, coffee, soft drinks, black tea, } \\
\text { sauces, mayonnaise and dressing }\end{array}$} & 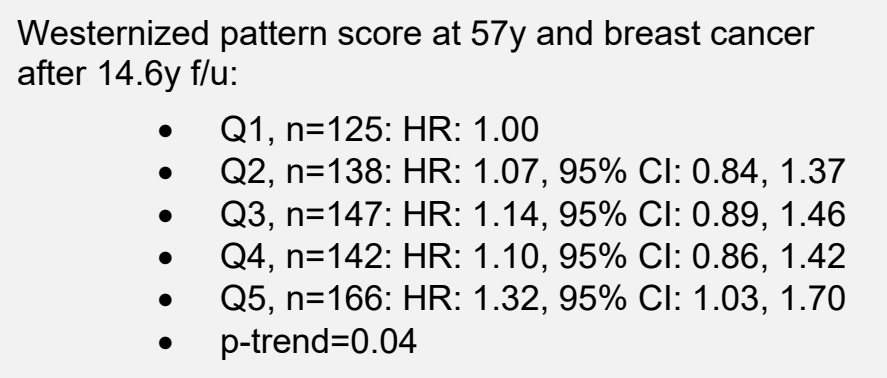 & \\
\hline & & $\begin{array}{l}\text { Results were similar when analyzed by quintiles among } \\
\text { the highest quintile group. }\end{array}$ & \\
\hline & & $\begin{array}{l}\text { When pre- and post-menopausal breast cancer were } \\
\text { analyzed separately, only post-menopausal breast } \\
\text { cancer risk was significantly associated with } \\
\text { westernized dietary pattern adherence. }\end{array}$ & \\
\hline & $\begin{array}{l}\text { Traditional Japanese pattern (PCA): Higher } \\
\text { loadings for salmon, seafood other than fish, } \\
\text { oily fish, lean fish, salty fish, chicken and } \\
\text { pickles }\end{array}$ & & $\begin{array}{l}\text { Traditional pattern score at } 57 y \text { was } \\
\text { not significantly associated with total, } \\
\text { premenopausal, or postmenopausal } \\
\text { breast cancer after } 14.6 y \text { f/u. }\end{array}$ \\
\hline
\end{tabular}




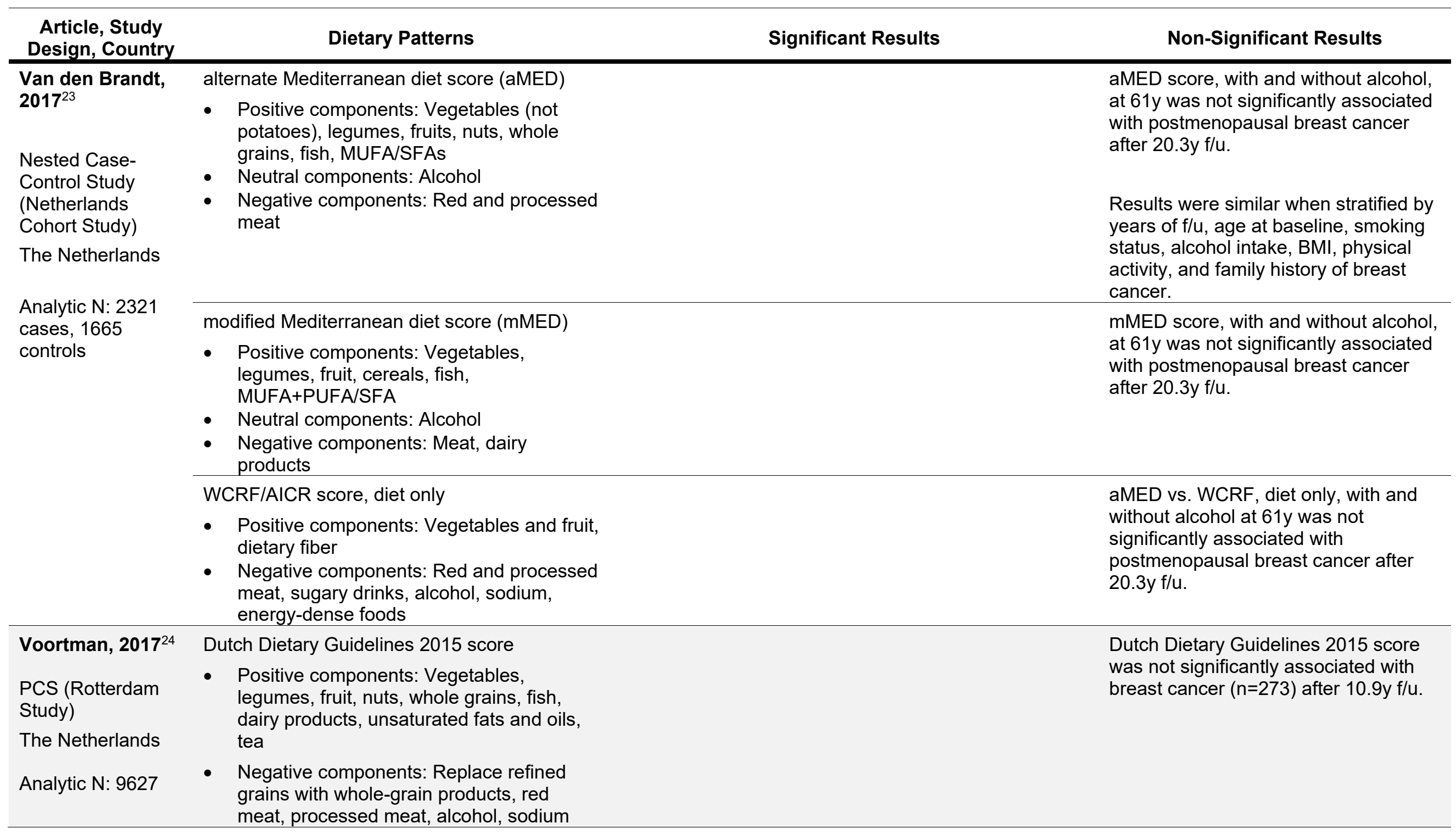




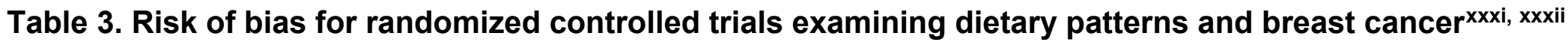

\begin{tabular}{|c|c|c|c|c|c|c|}
\hline & Randomization & $\begin{array}{c}\text { Deviations } \\
\text { from intended } \\
\text { interventions - } \\
\text { effect of } \\
\text { assignment }\end{array}$ & $\begin{array}{c}\text { Deviations } \\
\text { from intended } \\
\text { interventions - } \\
\text { per-protocol }\end{array}$ & $\begin{array}{c}\text { Missing } \\
\text { outcome data }\end{array}$ & $\begin{array}{c}\text { Outcome } \\
\text { measurement }\end{array}$ & $\begin{array}{l}\text { Selection of the } \\
\text { reported result }\end{array}$ \\
\hline Prentice, $2019^{1}$ & Low & Low & Some & Low & Low & Low \\
\hline Thomson, $2014^{2}$ & Low & Low & Some & Low & Low & Low \\
\hline Toledo, $2015^{3}$ & Some & Low & Low & Low & Low & Low \\
\hline
\end{tabular}

Table 4. Risk of bias for observational studies examining dietary patterns and breast cancer ${ }^{\mathrm{xx} x i i \mathrm{i}}$

\begin{tabular}{|c|c|c|c|c|c|c|c|}
\hline & Confounding & $\begin{array}{l}\text { Selection of } \\
\text { participants }\end{array}$ & $\begin{array}{l}\text { Classification } \\
\text { of exposures }\end{array}$ & $\begin{array}{c}\text { Deviations } \\
\text { from intended } \\
\text { exposures }\end{array}$ & Missing data & $\begin{array}{c}\text { Outcome } \\
\text { measurement }\end{array}$ & $\begin{array}{l}\text { Selection of the } \\
\text { reported result }\end{array}$ \\
\hline Catsburg, $2015^{25}$ & Serious & Serious & Low & Serious & Serious & Low & Serious \\
\hline Deschasaux, $2018^{4}$ & Serious & Moderate & Low & Serious & Moderate & Low & Moderate \\
\hline Deschasaux, $2017^{5}$ & Serious & Moderate & Low & Serious & Moderate & Low & Moderate \\
\hline Donnenfeld, $2015^{6}$ & Serious & Moderate & Low & Serious & Moderate & Low & Moderate \\
\hline Fiolet, $\mathbf{2 0 1 8}^{7}$ & Serious & Moderate & Moderate & Serious & Moderate & Low & Moderate \\
\hline Guinter, 2018a (IJC) ${ }^{8}$ & Serious & Serious & Moderate & Serious & Serious & Low & Serious \\
\hline Guinter, 2018b (CEBP) ${ }^{9}$ & Serious & Serious & Moderate & Serious & Serious & Low & Moderate \\
\hline
\end{tabular}

xxxi A detailed description of the methodology used for assessing risk of bias is available on the NESR website: https://nesr.usda.gov/2020-dietary-guidelinesadvisory-committee-systematic-reviews and in Part C of the following reference: Dietary Guidelines Advisory Committee. 2020. Scientific Report of the 2020

Dietary Guidelines Advisory Committee: Advisory Report to the Secretary of Agriculture and the Secretary of Health and Human Services. U.S. Department of Agriculture, Agricultural Research Service, Washington, DC.

xxxii Possible ratings of low, some concerns, or high determined using the "Cochrane Risk-of-bias 2.0" (RoB 2.0) (August 2016 version)" (Higgins JPT, Sterne JAC, Savović J, Page MJ, Hróbjartsson A, Boutron I, Reeves B, Eldridge S. A revised tool for assessing risk of bias in randomized trials In: Chandler J, McKenzie J, Boutron I, Welch V (editors). Cochrane Methods. Cochrane Database of Systematic Reviews 2016, Issue 10 (Suppl 1). dx.doi.org/10.1002/14651858.CD201601.)

xxxiii Possible ratings of low, moderate, serious, critical, or no information determined using the "Risk of Bias for Nutrition Observational Studies" tool (RoBNObs) (Dietary Guidelines Advisory Committee. 2020. Scientific Report of the 2020 Dietary Guidelines Advisory Committee: Advisory Report to the Secretary of Agriculture and the Secretary of Health and Human Services. U.S. Department of Agriculture, Agricultural Research Service, Washington, DC.) 


\begin{tabular}{|c|c|c|c|c|c|c|c|}
\hline Haridass, $2018^{10}$ & Moderate & Serious & Low & Serious & Serious & Low & Moderate \\
\hline Harris, $2015^{11}$ & Serious & Serious & Moderate & Serious & Moderate & Low & Moderate \\
\hline Harris, 2016 & Serious & Serious & Serious & Serious & Serious & Low & Serious \\
\hline Harris, $2017^{13}$ & Serious & Serious & Serious & Serious & Serious & Low & Serious \\
\hline Kane-Diallo, 2018 ${ }^{14}$ & Serious & Moderate & Low & Serious & Moderate & Low & Moderate \\
\hline Kojima, 2017' & Serious & Serious & Serious & Serious & Serious & Low & Moderate \\
\hline Lavalette, $2018^{16}$ & Serious & Moderate & Low & Serious & Serious & Low & Moderate \\
\hline Li, $2015^{17}$ & Serious & Moderate & Low & Serious & Moderate & Low & Moderate \\
\hline McKenzie, $2015^{18}$ & Serious & Moderate & Moderate & Serious & Serious & Low & Moderate \\
\hline Nomura, 2016 ${ }^{19}$ & Serious & Moderate & Low & Moderate & Moderate & Low & Moderate \\
\hline $\begin{array}{l}\text { Penniecook-Sawyers, } \\
2016^{20}\end{array}$ & Moderate & Serious & Low & Serious & Moderate & Low & Moderate \\
\hline Petimar, 201921 & Moderate & Moderate & Low & Serious & Serious & Low & Serious \\
\hline Pot, $2014^{26}$ & Serious & Moderate & Moderate & Serious & Moderate & No information & Moderate \\
\hline Shin, 2016 22 & Serious & Moderate & Moderate & Serious & Moderate & Low & Moderate \\
\hline Van den Brandt, $2017^{23}$ & Serious & Moderate & Low & Serious & Moderate & Low & Moderate \\
\hline Voortman, $2017^{24}$ & Serious & Moderate & Low & Serious & Moderate & Low & Moderate \\
\hline
\end{tabular}


Table 5. Description of studies that examined the relationship between dietary patterns and colorectal cancer ${ }^{\mathrm{xxxiv}}$

\begin{tabular}{|c|c|c|c|}
\hline $\begin{array}{l}\text { Study and } \\
\text { Participant } \\
\text { Characteristics }\end{array}$ & Intervention/Exposure and Outcomes & Results & $\begin{array}{l}\text { Confounding, Study Limitations, and } \\
\text { Summary of Findings }\end{array}$ \\
\hline \multicolumn{4}{|l|}{$\begin{array}{l}\text { Randomized } \\
\text { Controlled Trials }\end{array}$} \\
\hline Prentice, 2019 1 & $\begin{array}{l}\text { Dietary patterns: } \\
\text { - Intervention group: Reduction in fat from }\end{array}$ & Significant: N/A & $\begin{array}{l}\text { Key confounders accounted for: N/A for } \\
\text { RCTs }\end{array}$ \\
\hline $\begin{array}{l}\text { RCT (Women's Health } \\
\text { Initiative Dietary } \\
\text { Modification (DM) trial) } \\
\text { United States }\end{array}$ & $\begin{array}{l}\sim 35 \% \mathrm{E} \text { to } 20 \% \mathrm{E} \text { of energy, } 5 \text { servings/d } \\
\text { fruits and vegetables, } 6 \text { serving/d grains; } \\
\text { achieved reductions } 8-10 \% \mathrm{E} \text { of total, } \\
\text { saturated, and unsaturated fat, reductions } \\
\text { in } 8-10 \% \text { of carbohydrate; had higher }\end{array}$ & $\begin{array}{l}\text { Non-Significant: } \\
\text { There were no significantly differences } \\
\text { between intervention and comparison } \\
\text { groups and colorectal cancer during the }\end{array}$ & $\begin{array}{l}\text { Other: Baseline hazard stratified on age at } \\
\text { random assignment, ethnicity, hysterectomy } \\
\text { status, prior disease (if applicable), } \\
\text { randomization status in the hormone therapy } \\
\text { trials, and study phase }\end{array}$ \\
\hline $\begin{array}{l}\text { Analytic N: } 48835 \\
\text { (Intervention: } 19541 \text {, } \\
\text { Comparison: 29294) } \\
\text { (Attrition: 0\%) }\end{array}$ & $\begin{array}{l}\text { intakes of fruits, vegetables, whole grains, } \\
\text { and total grains } \\
\text { Comparison group: Received written health- } \\
\text { related materials only; lower intakes of } \\
\text { fruits, vegetables, whole grains, and total } \\
\text { arains }\end{array}$ & $\begin{array}{l}\text { Intervention, after 8.5y t/u, and after 19.6y } \\
\text { f/u. }\end{array}$ & $\begin{array}{l}\text { Limitations: } \\
\text { - The intensity of the intervention may have } \\
\text { differed between groups, as the } \\
\text { intervention group received more } \\
\text { intensive education than the comparison }\end{array}$ \\
\hline $\begin{array}{l}\text { Participants were } \\
100 \% \text { female, } \sim 62 \mathrm{y} \\
(50-79 \mathrm{y}), 28.2 \mathrm{~kg} / \mathrm{m} 2 \\
51 \% \text { never smokers }\end{array}$ & $\begin{array}{l}\text { Dietary assessment methods: Adherence was } \\
\text { monitored using FFQs at baseline, } 1 \mathrm{y} \text {, and } \\
\text { every } 3 y \text { thereafter }\end{array}$ & & $\begin{array}{l}\text { Summary: There were no difference between } \\
\text { intervention and comparison groups in risk of } \\
\text { colorectal cancer during the } 8.5 y \text { intervention } \\
\text { or over } 19.6 y \mathrm{f} / \mathrm{u} \text {. }\end{array}$ \\
\hline
\end{tabular}

xxxivAbbreviations : AHEI-2010, Alternative Healthy Eating Index-2010; aMED, Alternative Mediterranean Diet Score ; BMI, body mass index; CDQI, Colorectal Diet Quality Index; d, day; DASH, Dietary Approaches to Stop Hypertension; DFA, "High-dairy", "high-fruit-and-vegetable", "low, alcohol" dietary pattern; DM, dietary modification; DP, Dietary pattern; \%E, \% of energy; EDIH, Empirical dietary index for hyperinsulinemia; EDIP, Empirical dietary inflammatory pattern; EPIC, European Prospective Investigation into Cancer and Nutrition; FFQ, food frequency questionnaire; FSAm-NPS, Nutrient Profiling System of the British Food Standards Agency (modified version); f/u, follow-up; HNFI, Healthy Nordic food index; HR, hazard ratio; IMI, Italian Mediterranean Index; MEDI-LITE, Mediterranean diet score; MD, Mediterranean diet; MDS, Mediterranean Diet Score; mo, month(s); N/A, Not applicable; NIH, National Institutes of Health; NOVA, Ultra-processed food score; NS, Not significant; NR, Not reported; PCS, prospective cohort study; pt, point; RCT, randomized controlled trial; SEER, Surveillance, Epidemiology, and End Results Program; WCRF/AICR, World Cancer Research Fund/American Institute for Cancer Research; wk, week(s); y, year(s) 


\section{Study and}

Participant

Characteristics
Results
Confounding, Study Limitations, and

Summary of Findings

\begin{tabular}{|c|c|c|c|}
\hline & $\begin{array}{l}\text { Outcome assessment methods: US National } \\
\text { Cancer Institute's SEER system }\end{array}$ & & \\
\hline \multirow{7}{*}{$\begin{array}{l}\text { Thomson, 2014² } \\
\text { RCT (Women's Health } \\
\text { Initiative Dietary } \\
\text { Modification (DM) trial) } \\
\text { United States } \\
\text { Analytic N: } 48835 \\
\text { (Intervention: 19541, } \\
\text { Comparison: 29294) } \\
\text { (Attrition: 0\%) }\end{array}$} & \multirow{6}{*}{$\begin{array}{l}\text { Dietary patterns: } \\
\text { - Intervention group: Reduction in fat from } \\
\sim 35 \% \mathrm{E} \text { to } 20 \% \mathrm{E} \text { of energy, } 5 \text { servings/d } \\
\text { fruits and vegetables, } 6 \text { serving/d grains; } \\
\text { achieved reductions } 8-10 \% \mathrm{E} \text { of total, } \\
\text { saturated, and unsaturated fat, reductions } \\
\text { in } 8-10 \% \text { of carbohydrate; had higher } \\
\text { intakes of fruits, vegetables, whole grains, } \\
\text { and total grains } \\
\text { Comparison group: Received written health- } \\
\text { related materials only; lower intakes of } \\
\text { fruits, vegetables, whole grains, and total } \\
\text { grains } \\
\text { Dietary assessment methods: Adherence was } \\
\text { monitored using FFQs at baseline, } 1 \mathrm{y}, \text { and } \\
\text { every 3y thereafter }\end{array}$} & Significant: N/A & Key confounders accounted for: N/A for \\
\hline & & \multirow{5}{*}{$\begin{array}{l}\text { Non-Significant: } \\
\text { There were no significantly differences } \\
\text { between intervention and comparison } \\
\text { groups and colorectal cancer during the } \\
\text { intervention, after } 8.5 y \text { f/u, and after } 13.5 y \\
\text { f/u. }\end{array}$} & Other: N/A \\
\hline & & & Limitations: \\
\hline & & & $\begin{array}{l}\text { - The intensity of the intervention may have } \\
\text { differed between groups, as the } \\
\text { intervention group received more } \\
\text { intensive education than the comparison }\end{array}$ \\
\hline & & & Funding Sources: NIH \\
\hline & & & $\begin{array}{l}\text { Summary: There were no difference between } \\
\text { intervention and comparison groups in risk of } \\
\text { colorectal cancer during the } 8.5 y \text { intervention } \\
\text { or over } 13.5 y \mathrm{f} / \mathrm{u} \text {. }\end{array}$ \\
\hline & $\begin{array}{l}\text { Outcome assessment methods: US National } \\
\text { Cancer Institute's SEER system }\end{array}$ & & \\
\hline \multicolumn{4}{|l|}{$\begin{array}{l}\text { Observational } \\
\text { Studies }\end{array}$} \\
\hline Boden, $2019^{27}$ & Dietary patterns: & Significant: N/A & Key confounders accounted for: \\
\hline PCS (Vasterbotten & $\begin{array}{l}\text { - Mediterranean diet score (MDS) }{ }^{x \times x v} \text {, per } \\
\text { tertile increase }\end{array}$ & Non-Significant: & $\begin{array}{l}\text { Sex, age, education, alcohol intake, physical } \\
\text { activity, smoking, BMI }\end{array}$ \\
\hline Programme) & $\begin{array}{l}\text { Positive components: Vegetables and } \\
\text { potatoes, fruit and fresh juices, }\end{array}$ & & Other: \\
\hline
\end{tabular}

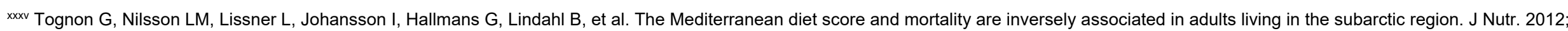
142 (8):1547-53. 


\section{Study and}

Participant

Characteristics

\section{Sweden}

Analytic N: 35393

Participants were 52\% female, $\sim 46 y$, 15\% obese, $\sim 17 \%$ current smoker

Excluded participants with prevalent cancer, insufficient dietary data, implausible food or energy intakes, implausible anthropometric data, cancer diagnosed within 1y year of last measurement, single dietary measure ( $65 \%$ of original sample)
Intervention/Exposure and Outcomes

wholegrain cereals, fish and fish
products, MUFA+PUFA/SFA,

○ Moderation components: Alcohol

- Negative components: Meat and meat products, dairy products

Dietary assessment methods: 84 -item and 64-66-item, validated, FFQs, at least 2 measures less than $2 y$ apart at baseline, age $\sim 46 \mathrm{y}$

Outcome assessment methods: Swedish

Cancer Registry

\section{Results}

Mediterranean diet score at 46y was not significantly associated with colorectal cancer $(n=1036)$ after 15y $\mathrm{f} / \mathrm{u}$ in all participants, or when men and women were analyzed separately.
Confounding, Study Limitations, and Summary of Findings

\section{Energy intake}

\section{Limitations:}

- Did not account for race/ethnicity, family history of the cancer outcome,

inflammatory bowel disease, colorectal polyps

- Only assessed dietary intake during the first year of f/u; did not account for possible changes in dietary intake over $\mathrm{f} / \mathrm{u}$

- Did not account for missing data

- $\quad$ No preregistered statistical plans; potential for selective outcome reporting

Funding Sources: The Cancer Research Fund in Northern Sweden, Arctic Research Center at Umeå University, Ostersunds Hospital, Swedish Cancer Society, Region Vasterbotten, Swedish Research Council for Health, Working Life and Welfare, Swedish Research Council

Summary: Mediterranean diet score at 46y was not significantly associated with risk of colorectal cancer after $15 \mathrm{y} / \mathrm{f}$.

\section{Key confounders accounted for:}

Sex, age, race/ethnicity, education, physical activity, smoking, BMI, family history of the cancer outcome

\section{PCS (lowa Women's Health Study)}

\section{Dietary patterns:} categorical and continuous o Positive components: Vegetables,

\section{Significant: N/A}

Non-Significant:

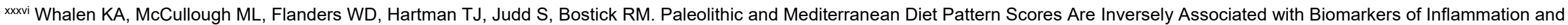
Oxidative Balance in Adults. J Nutr 2016;146(6):1217-26. 


\section{Study and}

Participant

Characteristics

United States

Analytic N: 35221

Participants were $100 \%$ female, $\sim 61$ yo (55-69y), 27kg/m2 BMI, 33\% ever smokers, $\sim 3.5 \mathrm{~g} / \mathrm{d}$ alcohol, 14\% high physical activity

Excluded those with history of cancer, missing dietary record data, implausible energy intake ( 16\% of original sample)

Deschasaux, 2018

\section{PCS (European}

Prospective

Investigation into

Cancer and Nutrition

(EPIC))

Denmark, France,

Germany, Greece,
Intervention/Exposure and Outcomes

fruits, lean meats, fish, nuts, fruit and

vegetable diversity, calcium

- Negative components: Red and processed meats, sodium, dairy foods, grains and starches, baked goods, sugar-sweetened beverages, alcohol

- Mediterranean diet score ${ }^{\mathrm{xxxvii}}$, categorical

- Positive components: Vegetables (not potatoes), legumes, fruits, nuts, whole grains, fish, MUFA/SFAs

- Neutral components: Alcohol

- Negative components: Red and processed meat

Dietary assessment methods: 127-item, validated FFQ at baseline, $\sim 61 \mathrm{y}$

Outcome assessment methods: State Health Registry of lowa, National Death Index

\section{Dietary patterns:}

Nutrient Profiling System of the British Food Standards Agency (modified version) (FSAmNPS) score, categorical (quintiles) and continuous (per 2 pt increment)

- Overall diet score assigned based on energy, sugar, saturated fatty acid, sodium, fibers, proteins, and fruits/vegetables/legumes/nuts.

\section{Results}

Evolutionary-concordance score at 61y (categorical and continuous) was not significantly associated with colorectal cancer $(n=1731)$ after 18y f/u.

Mediterranean diet score at 61y (categorical and continuous) was not significantly associated with colorectal cancer $(n=1731)$ after $18 y$ f/u.
Confounding, Study Limitations, and Summary of Findings

\section{Other:}

Total energy intake, hormone replacement therapy, arthritis

\section{Limitations:}

- Did not account for inflammatory bowe disease, colorectal polyps

- Only assessed dietary intake once at baseline; did not account for possible changes in dietary intake over $\mathrm{f} / \mathrm{u}$

- Did not fully account for missing data

- No preregistered statistical plans; potential for selective outcome reporting

Funding Sources: NIH

Summary: Evolutionary-concordance and Mediterranean diet scores at $61 y$ were not associated with risk of colorectal over a $18 y$ period of $f / u$.

\section{Significant:}

FSAm-NPS score at 51y and risk of colorectal cancer after $15.3 \mathrm{y} / \mathrm{u}$ :

- Q1, n=1144: HR: 1.00

- Q2, n=1150: HR: $1.07,95 \% \mathrm{Cl}$ $0.99,1.17$

\section{Key confounders accounted for:}

Sex, age, education, alcohol intake, physical activity, smoking, BMI, height, family history of the cancer

\section{Other:}

- Q3, n=1152: HR: $1.07,95 \% \mathrm{Cl}$ $0.98,1.17$

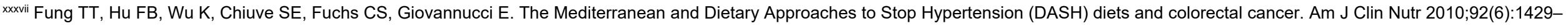
35. 


\section{Study and}

Participant

Characteristics

\section{Ttaly, the}

Netherlands,

Norway, Spain,

Sweden, UK

Analytic N: 471495

Participants were $70 \%$

female, 51yo, 25.4

$\mathrm{kg} / \mathrm{m}^{2}, 43 \%$ neversmokers, $\sim 5.3 \mathrm{~g} / \mathrm{d}$

alcohol

Excluded those with prevalent cancer; cancer diagnosis in first $2 y$ of $f / u$; missing data; implausible energy intake ( 10\% of original sample)

\section{Intervention/Exposure and Outcomes Results}

- Higher FSAm-NPS score had higher intakes of alcohol, energy and red and processed meat, lower intakes of dietary fibers, vegetables, fruit, fish, and lean meat

Dietary assessment methods: FFQs or 7-day diet records, validated, at baseline, age $\sim 51 \mathrm{y}$

Outcome assessment methods: Record linkage with population-based cancer registries, health insurance records, pathology registries, and f/u with study participants
- Q4, $\mathrm{n}=1195: \mathrm{HR}: 1.12,95 \% \mathrm{Cl}:$ $1.02,1.22$

- Q5, $\mathrm{n}=1165: \mathrm{HR}: 1.11,95 \% \mathrm{Cl}$ $1.01,1.22$

- $\quad$-trend $=0.02$

Continuous, per $2 \mathrm{pt}$ increment, $\mathrm{n}=5086$ cases: HR: $1.03,95 \% \mathrm{Cl}: 1.00,1.06, \mathrm{p}-$ trend $=0.03$

\section{Non-Significant: N/A}

Confounding, Study Limitations, and Summary of Findings

Center, hormone replacement therapy, age at menarche, age at first full-term pregnancy, age at menopause, energy intake

\section{Limitations:}

- Did not account for race/ethnicity, inflammatory bowel disease, colorectal polyps

- Only assessed dietary intake once at baseline; did not account for possible changes in dietary intake over $\mathrm{f} / \mathrm{u}$

- Did not fully account for missing data

- No preregistered statistical plans; potential for selective outcome reporting

Funding Sources: French National Cancer Institute, European Commission, the International Agency for Research on Cancer

Summary: Consuming a diet that scores higher on the Nutrient Profiling System of the British Food Standards Agency (modified version) (FSAm-NPS) at 51y was associated with increased risk of colorectal cancer after $15.3 y \mathrm{f} / u$.

Fasanelli, 2017
PCS (European
Prospective
Investigation into
Cancer and
Nutrition)

\section{Dietary patterns:}

- Italian Mediterranean Index (IMI)xxxvii,, categorical, via mediation analysis with waist-to-hip ratio

- Positive components: Pasta, Mediterranean vegetables (raw

\section{Significant:}

Total causal effect of IMI score at 50y and colorectal cancer $(n=414)$ after $11 \mathrm{y} f / \mathrm{u}$ :

- 0-1: HR: 1.00

- 2-3: HR: 0.51, 95\% Cl: 0.95, 0.35 0.84
Key confounders accounted for: NR (the mediation analysis required several assumptions, including no unmeasured exposure-mediator, mediator-outcome and exposure-outcome confounding; and no effect of any exposure that may confound the mediator-outcome relationship) 


\section{Study and}

Participant

Characteristics

\section{Italy}

\section{Analytic N: 42984}

Participants were $70 \%$ female, $\sim 50$ yo, $55 \%$ overweight or obese, $\sim 47 \%$ never smokers, $\sim 68 \%$ inactive

\section{Excluded those with} prevalent cancers, missing information on diet, anthropometrics, or lifestyle, implausible energy intake $(\sim 10 \%$

of original sample)

\section{Intervention/Exposure and Outcomes}

tomatoes, cooked leafy vegetables, raw leafy vegetables, onion or garlic, mixed salad or mixed vegetables), fruits, legumes, olive oil, fish

- Neutral components: Alcohol

- Negative components: Soft drinks, butter, red meat, potatoes

Dietary assessment methods: Validated, FFQ at baseline, at $50 \mathrm{y}$

\section{Outcome assessment methods: NR}

Confounding, Study Limitations, and Summary of Findings
- 4-5: HR: $0.62,95 \%$ Cl: $0.95,0.41$, 0.95

- 6-1: HR: 0.51, 95\% Cl: 0.95, 0.31, 0.85

Results of the pure direct effect analysis were similar. However, results were not significantly when analyzed through waistto-hip ratio, via the natural indirect effect analysis.

\section{Non-Significant: N/A}

\section{Dietary patterns:}

- Ultra-processed food score (NOVA), categorical (quartiles)

\section{PCS (NutriNet-Santé)}

\section{France}

Analytic N: 104,980
- Main food groups contributing to NOVA score were sugary drinks, drinks, starchy foods and breakfast cereals, ultra-processed fruits and vegetables,

\section{Significant: N/A}

\section{Non-Significant}

Ultra-processed food score at $49 y$ and colorectal cancer after $5.4 \mathrm{y} / \mathrm{u}$ :

- Q1, n=48: HR: 1.00

\section{Other: N/A}

\section{Limitations:}

- Unclear whether the following key confounders were accounted for: sex, age, race/ethnicity, socioeconomic status, alcohol intake (in adults), physical activity, smoking, anthropometry, family history of the cancer outcome, inflammatory bowel disease, colorectal polyps

- Only assessed dietary intake once at baseline; did not account for possible changes in dietary intake over f/u

- Did not fully account for missing data

- No preregistered statistical plans; potential for selective outcome reporting

Funding Sources: Italian Ministry of Health, the Italian Association for Cancer Research, the Compagnia di San Paolo

Summary: Higher adherence to the Italian Mediterranean Index at 50y was significantly associated with decreased risk of colorectal cancer after $11 \mathrm{y} / \mathrm{u}$. This was independent of waist-to-hip ratio.

\section{Key confounders accounted for:}

Sex, age, education, alcohol intake, physical activity, smoking, BMI, height, family history of the cancer

\section{Other:}

Energy intake without alcohol, number of 24 hour dietary records, intakes of lipids, sodium, 


\section{Study and \\ Participant Characteristics \\ Participants were $78 \%$ female, 42.8 yo, $23.8 \mathrm{~kg} / \mathrm{m} 2 \mathrm{BMI}, 83 \%$ never or former smokers, $\sim 7.8 \mathrm{~g} / \mathrm{d}$ achohol \\ Excluded those with prevalent cancer; $<35$ yo at baseline; at least 2 valid $24-\mathrm{hr}$ dietary records during first $2 \mathrm{y} \mathrm{f} / \mathrm{u}$; diagnosis in first $2 y$ of $\mathrm{f} / \mathrm{u}(\sim 40 \%$ of original sample)}

Intervention/Exposure and Outcomes

Results dairy products, meats, fish, and eggs,

processed meats, fats, and salty snacks

Dietary assessment methods: 3, 24-hour recalls, assessed during the first year of $f / u$, at age $\sim 49 y$

\section{Outcome assessment methods:}

Participant report, medical record review, pathological reports
- Q2, $n=43: H R: 1.12,95 \%$ Cl: 0.74, 1.70

- Q3, n=36: HR: $1.22,95 \% \mathrm{Cl}: 0.79$, 1.89

- Q4, n=26: HR: $1.23,95 \% \mathrm{Cl}: 1.08$, 1.40

- $\quad$-trend $=0.07$

Ultra-processed food score, continuous, and colorectal cancer $(n=153)$ : HR: 1.16, 95\% Cl: 0.95, 1.42; p-trend=0.10
Confounding, Study Limitations, and Summary of Findings pattern

\section{Limitations:}

- Did not account for inflammatory bowel disease or colorectal polyps

- Only assessed dietary intake during the first year of f/u; did not account for possible changes in dietary intake over $\mathrm{f} / \mathrm{u}$

- Did not fully account for missing data

- No preregistered statistical plans; potential for selective outcome reporting

Funding Sources: Région lle-de-France, Cancéropôle lle-de-France, Ministère de la Santé, Institut de Veille Sanitaire, Institut National de la Prévention etde l'Education pour la Santé, Institut Nationalde la Santé et de la Recherche Médicale, Institut National de la Recherche Agronomique, onservatoire National des Arts et Métiers, Université Paris 13

Summary: Ultra-processed food score at $49 y$ was not significantly associated with risk of colorectal cancer after $5.4 \mathrm{y} / \mathrm{u}$.

\section{Key confounders accounted for:}

Sex, age, alcohol intake, physical activity, smoking, BMI, family history of the cancer outcome
PCS (UK Women's Cohort Study) and continuous (per 2pt increment)

\section{Significant:}

Mediterranean diet score at 52y and colorectal cancer after $17.4 \mathrm{y}$ f/u

- Q1, n=74, HR: 1.00

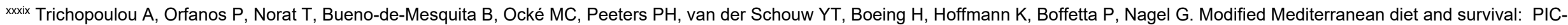
elderly prospective cohort study. Bmj. 2005 Apr 28;330(7498):991. 


\section{Study and}

Participant

Characteristics

\section{United Kingdom}

Analytic N: 35372

Participants were $100 \%$ female, 52 yo, $24 \mathrm{~kg} / \mathrm{m} 2 \mathrm{BMI}, \sim 11 \%$ current smokers, $\sim 5 \mathrm{~g} / \mathrm{d}$ alcohol, $\sim 0.2 \mathrm{hr}$ physical activity/d

Excluded those with prevalent or history of cancer, diagnosed with colorectal cancer within $1 \mathrm{y}$ of baseline, missing dietary records and covariate data, implausible energy intake ( $\sim 9 \%$ of original sample)

\section{Intervention/Exposure and Outcomes}

- Positive components: Vegetables, legumes, fruit, cereals, fish, MUFA+PUFA/SFA

- Neutral components: Alcohol

- Negative components: Meat, dairy products

Dietary assessment methods: 217-item, validated FFQ at baseline, $\sim 52 \mathrm{y}$

Outcome assessment methods: Record linkage with the National Health Service
Results

- $\quad$ Q2, $n=75, H R: 0.91,95 \%$ Cl: 0.64, 1.30

- $Q 3, n=88, H R: 0.82,95 \% \mathrm{Cl}: 0.58$ 1.15

- $\mathrm{Q} 4, \mathrm{n}=136, \mathrm{HR}: 0.63,95 \% \mathrm{Cl}: 0.45$, 0.87

- $Q 5, n=92, H R: 0.82,95 \% \mathrm{Cl}: 0.57$ 1.17

- Per 2 unit increment: HR: $0.88,95 \%$ Cl: $0.78,0.99$

- $p$-trend $=0.030$

Mediterranean diet score at 52y and rectal cancer after $17.4 \mathrm{y} / \mathrm{u}$ :

- Q1, n=30, HR: 1.00

- Q2, $\mathrm{n}=26, \mathrm{HR}: 0.77,95 \% \mathrm{Cl}: 0.44$, 1.35

- Q3, n=26, HR: $0.58,95 \% \mathrm{Cl}: 0.32$ 1.02

- Q4, n=44, HR: $0.50,95 \% \mathrm{Cl}: 0.29$ 0.83

- $\mathrm{Q} 5, \mathrm{n}=28, \mathrm{HR}: 0.38,95 \% \mathrm{Cl}: 0.20$, 0.74

- Per 2 unit increment: HR: 0.69, 95\% $\mathrm{Cl}: 0.56,0.86$

- $p$-trend $=0.001$

\section{Non-Significant:}

Mediterranean diet score at 52y was not significantly associated with colon, proximal colon, or distal colon cancer after $17.4 \mathrm{y} f / u$.
Confounding, Study Limitations, and Summary of Findings

\section{Other:}

Energy intake

\section{Limitations:}

- Did not account for race/ethnicity, socioeconomic status, inflammatory bowel disease, colorectal polyps

- Only assessed dietary intake once at baseline; did not account for possible changes in dietary intake over $\mathrm{f} / \mathrm{u}$

- Did not account for missing data

- No preregistered statistical plans; serious potential for selective outcome reporting

Funding Sources: World Cancer Research Fund

Summary: Higher adherence to the mediterranean diet score at $52 y$ was significantly associated with lower risk of colorectal and rectal cancer after $17.4 \mathrm{y} / \mathrm{u}$. Mediterranean diet score at 52y was not significantly associated with colon, proximal colon, or distal colon cancer after $17.4 y \mathrm{f} / \mathrm{u}$. 


\section{Study and \\ Participant \\ Characteristics \\ Kumagai, 2014 30 \\ PCS (Ohsaki \\ National Health \\ Insurance (NHI) \\ Cohort Study) \\ Japan}

Analytic N: 44079

Participants were $\sim 46 \%$ female, $\sim 60 \mathrm{y}$ (40-79y), $\sim 23.6 \mathrm{~kg} / \mathrm{m} 2$ BMI, $32 \%$ current smokers, $\sim 48 \%$ current drinkers

\section{Excluded those} without history of cancer at baseline, missing dietary or outcome data, implausible energy intakes $(\sim 20 \%$ of original sample)

\section{Dietary patterns:}

Adherence to 3 dietary patterns identified using factor analysis (principal component analysis), categorical:

- “Japanese" dietary pattern: Higher loadings for soybean products, fish, seaweeds, vegetables, fruits, and green tea

- "Animal food" dietary pattern: Higher loadings for beef pork, ham, sausage, chicken, liver, butter, coffee, and alcoholic beverages

- "High-dairy", "high-fruit-and-vegetable", "low, alcohol" (DFA) dietary pattern: Higher loadings for dairy products (milk and yoghurt), margarine, fruits, and vegetables (carrot, pumpkin and tomato), and lower for rice, miso soup, and alcoholic beverages

Dietary assessment methods: 40 -item, validated FFQ at baseline, $\sim 60 \mathrm{y}$

Outcome assessment methods: Miyagi

Prefectural Cancer Registry

\section{Results}

\section{Significant:}

DFA pattern score at $60 \mathrm{y}$ and colorectal cancer after 11y f/u:

- $Q 1, n=288: H R: 1.00$

- Q2, $n=223:$ HR: $0.88,95 \% \mathrm{Cl}$ : $0.72,1.06$

- Q3, n=185: HR: $0.82,95 \% \mathrm{Cl}$ : $0.66,1.03$

- Q4, n=158: HR: $0.76,95 \% \mathrm{Cl}$ : $0.60,0.97$

- $\mathrm{p}$-trend $=0.02$

DFA pattern score at $60 y$ and rectal cancer after 11y f/u:

- $Q 1, n=127: H R: 1.00$

- Q2, $\mathrm{n}=82$ : HR: $0.79,95 \% \mathrm{Cl}: 0.58$, 1.07

- Q3, n=68: HR: $0.76,95 \% \mathrm{Cl}: 0.53$ 1.08

- Q4, n=46: HR: $0.56,95 \% \mathrm{Cl}: 0.37$ 0.84

- $\quad p$-trend $=0.0003$

\section{Non-Significant:}

"Japanese" dietary pattern and "animal food" dietary pattern scores at 60y were not significantly associated with risk of colorectal cancer after $11 \mathrm{y}$ f/u.

"Japanese" dietary pattern, "animal food", and DFA dietary pattern scores at $60 y$
Confounding, Study Limitations, and Summary of Findings

\section{Key confounders accounted for:}

Sex, age, education, alcohol intake (in adults), physical activity, smoking, BMI, family history of the cancer outcome

Other:

Total energy intake

\section{Limitations:}

- Did not account for race/ethnicity, inflammatory bowel disease, colorectal polyps

- Only assessed dietary intake once at baseline; did not account for possible changes in dietary intake over $\mathrm{f} / \mathrm{u}$

- Did not account for missing data

- No preregistered statistical plans; potential for selective outcome reporting

Funding Sources: Ministry of Health, Labour and Welfare, Japan

Summary: Higher adherence to the DFA dietary pattern at 60y was significantly associated with lower risk of colorectal and rectal cancer after $11 y \mathrm{f} / \mathrm{u}$.

"Japanese" dietary pattern and "animal food" dietary pattern scores at 60y were not significantly associated with risk of colorectal cancer after $11 y \mathrm{f} / \mathrm{u}$.

"Japanese" dietary pattern, "animal food", and DFA dietary pattern scores at 60y were not 


\section{Study and}

Participant

Characteristics

\section{Lavalette, $2018^{16}$ \\ PCS (NutriNet-Sante study)}

\section{France}

Analytic N: 41543

Participants were \% female, $\sim 54$ yo (all $>40 \mathrm{y}), \sim 24.5 \mathrm{~kg} / \mathrm{m} 2$, $\sim 44 \%$ never-smokers, $\sim 9.4 \mathrm{~g} / \mathrm{d}$ alcohol

Excluded those with prevalent cancer; $<3$ 24-hr recalls within the first year of $f / u$; missing f/u data; implausible energy intake; $<40 \mathrm{y}(>50 \%$ of original sample)

\section{Dietary patterns:}

- Alternate Healthy Eating Index 2010 (AHEI$2010)^{\times l}$, categorical (quintiles) and continuous (per 2 pt increment)

- Positive components: Vegetables (not potatoes, French fries), Fruit, Whole Grains, Long-Chain Fats (EPA + DHA), PUFA

- Neutral components: Alcohol

- Negative components: Red and Processed Meat, Sugar Sweetened Beverages and Fruit Juice, Trans FA, Sodium

- Mediterranean diet score (MEDI-LITE) ${ }^{\mathrm{xli}}$, categorical (quintiles) and continuous (per 2 pt increment)

- Positive components: Vegetables, legumes, fruit and nuts, cereals, fish, olive oil

- Neutral components: Alcohol

\section{Results}

were not significantly associated with risk

of colon cancer $(n=554)$ after $11 \mathrm{y} / \mathrm{u}$.

"Japanese" dietary pattern and "animal food" dietary pattern scores at 60y were not significantly associated with risk of rectal cancer $(n=323)$ after $11 \mathrm{y} / \mathrm{u}$.

\section{Confounding, Study Limitations, and} Summary of Findings

significantly associated with risk of colon cancer $(n=554)$ after $11 \mathrm{y} / \mathrm{f}$.

"Japanese" dietary pattern and "animal food" dietary pattern scores at 60y were not significantly associated with risk of rectal cancer $(n=323)$ after 11y f/u.

\section{Significant:}

Higher PNNS-GS score at $\sim 49 y$ (continuous) and colorectal cancer after 8.5y f/u: HR: 0.89, 95\% Cl: 0.80, 1.00, $\mathrm{p}=0.04$

\section{Non-Significant:}

AHEI-2010 score at $\sim 49 y$ (categorical and continuous) was not significantly associated with colorectal cancer after $8.5 y$ f/u.

MEDI-LITE score at $\sim 49 y$ (categorical and continuous) was not significantly associated with colorectal cancer after 8.5y f/u.

PNNS-GS score at $\sim 49 y$ (categorical) was not associated with risk of colorectal cancer after $8.5 \mathrm{y} / \mathrm{u}$.

\section{Key confounders accounted for:}

Sex, age, education, alcohol intake, physical activity, smoking, height, BMI, family history of the cancer

Other: Number of 24-hours dietary records, energy intake without alcohol, number of biological children, hormone replacement therapy

\section{Limitations:}

- Did not account for race/ethnicity, inflammatory bowel disease, colorectal polyps

- Only assessed dietary intake during the first year of f/u; did not account for possible changes in dietary intake over $\mathrm{f} / \mathrm{u}$

- Did not account for missing data

- $\quad$ No preregistered statistical plans; potential for selective outcome reporting

Funding Sources: Ministere de la Sante, Institut de Veille Sanitaire, Institut National de

${ }^{x l}$ Chiuve SE, Fung TT, Rimm EB, Hu FB, McCullough ML, Wang M, et al. Alternative dietary indices both strongly predict risk of chronic disease. J Nutr 2012;142:1009-18.

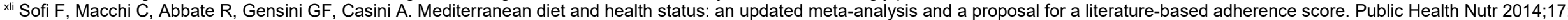
2769-82. 


\section{Study and}

Participant

Characteristics

\section{Results}

Confounding, Study Limitations, and Summary of Findings
○ Negative components: Meat, dairy products

- French National Nutrition Health ProgramGuideline Score (PNNS-GS) $)^{\text {liii }}$, categorical (quintiles) and continuous (per $2 \mathrm{pt}$ increment)

- Positive components: Vegetables and Fruit, Seafood, Vegetable Fat

- Neutral components: Breads, cereals, potatoes, legumes, meat and poultry, seafood, and eggs, milk and dairy products, alcohol

- Negative components: Sweetened foods, soda, added fat, salt

Dietary assessment methods: 3, 24-hour recalls, assessed during the first year of $f / u$, at age $\sim 49 y$

\section{Outcome assessment methods:}

Participant report, medical record review, pathological reports

Liu, $2017^{31}$
PCS (Nurses' Health
Study, Health
Professional Follow-
up Study)

\section{Dietary patterns:}

Adherence to a dietary pattern derived using lower rank regression (response variables: IL6, CRP (C-reactive protein) and TNFRSF1B (TNFa-receptor 2)):

- Empirical dietary inflammatory pattern (EDIP) score: Higher in red and processed

\section{Significant:}

EDIP score at $52 y$ and colorectal cancer after 2,998,258 person-y f/u:

- Q1: HR: 1.00

- Q2: HR: 0.86, 95\% Cl: 0.75, 0.98

- Q3: HR: 0.93, 95\% Cl: 0.82, 1.06 la Prevention et de l'Education pour la Sante, Region Ile-de-France, Institut National de la Sant_e et de la Recherche Medicale, Institut National de la Recherche Agronomique,

Conservatoire National des Arts et Metiers,

The French National Cancer Institute

Summary: Higher PNNS-GS score (continuous only) at $49 y$ were associated with significantly lower risk of colorectal cancer after $8.5 \mathrm{y} f / \mathrm{u}$. AHEI-2010 and MEDI-LITE scores at $49 y$ were not significantly associated with risk of colorectal cancer after $8.5 y \mathrm{f} / \mathrm{u}$.

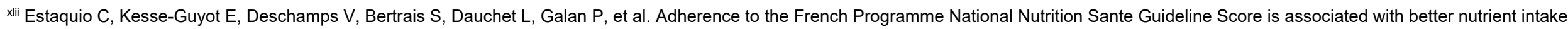
and nutritional status. J Am Diet Assoc 2009;109:1031-41. 


\section{Study and}

Participant

Characteristics

\section{United States}

Analytic N: 124,433

Participants were male and female, $52 \mathrm{yo}$, $\sim 25 \mathrm{~kg} / \mathrm{m} 2$ BMI, 12 $\sim 9 \mathrm{~g} / \mathrm{d}$ alcohol, $\sim 23$ METS-hrs/wk

Exclude those with ulcerative colitis, without birth dates, prevalent cancer before 1984, died before 1984, missing diet data ( $28 \%$ of original sample) pack-years smoking,

\section{Intervention/Exposure and Outcomes}

meats, refined grains, carbonated

beverages, and some vegetables; lower in beer, wine, coffee, tea, yellow and leafy vegetables, and fruit juice

Dietary assessment methods: Validated, FFQ at baseline and every $4 \mathrm{y}$ after, baseline at $52 \mathrm{y}$

Outcome assessment methods: F/u with participantes, medical record review, National Death Index, next of kin (Note: This paper also examined various molecular subtypes of colorectal cancer; results for molecular subtypes were not extracted in this table)
Results

- Q4: HR: 0.99, 95\% Cl: 0.87, 1.13

- Q5: HR: $1.14,95 \%$ Cl: 0.99, 1.30

- $p$-trend $=0.02$

\section{Non-Significant: N/A}

\section{Significant:}

\section{Dietary patterns:}

Adherence to 2 dietary patterns identified via principal component analysis:

PCS (Nurses' Health Study, Health

Professional F/u Study)

United States

Analytic N: 137217
- "Western" dietary pattern: Higher loadings for red and processed meats, high-fat dairy products (such as whole milk and cream), refined grains, and desserts

- "Prudent" dietary pattern: Higher loadings for vegetables, fruits, whole grains, and fish
"Western" dietary patterns at 52y and colorectal cancer after $32 \mathrm{y}$ f/u

- Q1, n=835: HR: 1.00

- Q2, n=822: HR: $1.09,95 \% \mathrm{Cl}$ : $0.99,1.20$

- Q3, n=784: HR: $1.11,95 \% \mathrm{Cl}$ : $1.00,1.24$

- Q4, n=819: HR: $1.31,95 \% \mathrm{Cl}$ : $1.15,1.48$

Confounding, Study Limitations, and Summary of Findings

Endoscopy status, Total energy intake, multivitamin use, aspirin use

\section{Limitations:}

- Did not account for race/ethnicity, socioeconomic status, anthropometry, colorectal polyps

- Did not fully account for missing data

- No preregistered statistical plans; potential for selective outcome reporting

Funding Sources: $\mathrm{NIH}$, Dana-Farber Harvard Cancer Center; The Paula and Russell Agrusa Fund for Colorectal Cancer Research, The Friends of the Dana-Farber Cancer Institute, Bennett Family Fund, and the Entertainment Industry Foundation through National Colorectal Cancer Research Alliance.

Summary: Higher empirical dietary inflammatory pattern score at 52y was associated with significantly increased risk of colorectal cancer.

\section{Key confounders accounted for:}

Sex, age, physical activity, smoking, BMI, family history of the cancer outcome

Other:

Calendar year, History of endoscopy, regular aspirin or NSAID use, total energy intake

\section{Limitations:}

- Did not account for race/ethnicity, 


\section{Study and}

Participant

Characteristics

Participants were $65 \%$

female, $\sim 60 \mathrm{yo}$,

$\sim 26 \mathrm{~kg} / \mathrm{m} 2 \mathrm{BMI}, \sim 8 \%$

women and $\sim 16 \%$

men were current

smokers, $\sim 8 \mathrm{~g} / \mathrm{d}$

alcohol

Exclude those with ulcerative colitis,

without birth dates,

prevalent cancer

before 1984, died

before 1984, missing

diet data $(\sim 5 \%$ of

original sample)
Intervention/Exposure and Outcomes

\section{Results}

- $p$-trend $=<0.0001$

Dietary assessment methods: Validated, FFQ at baseline and every $4 y$ after, baseline at $52 y$

Outcome assessment methods: F/u with participantes, medical record review, National Death Index, next of kin (Note: This paper and another by the same author also examined various molecular subtypes of colorectal cancer; results for molecular subtypes were not extracted in this table) xliii $0.90,1.34$ $0.97,1.47$
Confounding, Study Limitations, and Summary of Findings

socioeconomic status, alcohol intake, inflammatory bowel disease, colorectal polyps

"Western" dietary patterns at 52y and distal colon cancer after $32 \mathrm{y} / \mathrm{u}$ :

- Q1, n=216: HR: 1.00

- $Q 2, n=208: H R: 1.10,95 \% \mathrm{Cl}$ :

- $\quad \mathrm{Q} 3, \mathrm{n}=208: \mathrm{HR}: 1.19,95 \% \mathrm{Cl}$ :

- Q4, n=234: HR: $1.55,95 \% \mathrm{Cl}$ : 1.22, 1.96

- $\quad p$-trend $=0.0004$

"Western" dietary patterns at 52y and rectal cancer after $32 \mathrm{y} f / \mathrm{u}$ :

- $Q 1, n=172: H R: 1.00$

- Q2, $\mathrm{n}=151: \mathrm{HR}: 0.98,95 \% \mathrm{Cl}$ : $0.78,1.22$

- Q3, $n=176: H R: 1.24,95 \% \mathrm{Cl}$ : $0.99,1.56$

- $Q 4, n=171: H R: 1.35,95 \% \mathrm{Cl}$ : $1.03,1.77$

- $p$-trend $=0.01$

"Prudent" dietary patterns at 52y and colorectal cancer after $32 \mathrm{y} / \mathrm{u}$ :
Did not fully account for missing data

- No preregistered statistical plans; potential for selective outcome reporting

Funding Sources: NIH, Dana-Farber Harvard Cancer Center, The Project P Fund for Colorectal Cancer Research, The Friends of the Dana-Farber Cancer Institute, Bennett Family Fund, and the Entertainment Industry Foundation through National Colorectal Cancer Research Alliance

Summary: Higher adherence to a "western" dietary pattern at 52y was significantly associated with increased risk of colorectal, distal colon, and rectal cancer after $32 y \mathrm{f} / \mathrm{u}$. However, it was not significantly associated with proximal colon cancer. Results for the "western" dietary pattern were similar when men and women were analyzed separately.

Higher adherence to a "prudent" dietary pattern at 52y was significantly associated with lower risk of colorectal cancer after 32y f/u. However, it was not significantly associated with proximal colon, distal colon, or rectal cancer. Results for the "prudent"

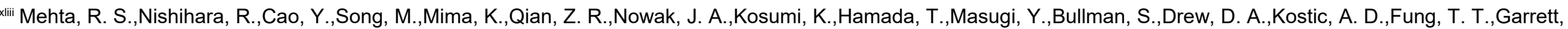

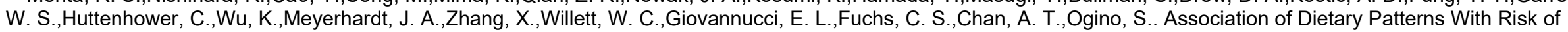
Colorectal Cancer Subtypes Classified by Fusobacterium nucleatum in Tumor Tissue. JAMA Oncol. 2017. 3:921-927 


\section{Study and}

Participant

Characteristics
Orlich, $2015^{33}$

\section{Dietary patterns:}

\section{PCS (Adventist}

\section{Results}

- Q2, n=761: HR: 0.88, 95\% Cl:

$$
0.80,0.97
$$

- Q3, n=830: HR: $0.90,95 \% \mathrm{Cl}$ : $0.82,1.00$

- Q4, n=826: HR: $0.86,95 \% \mathrm{Cl}$ : $0.77,0.95$

- $\quad p$-trend $=0.01$

\section{Non-Significant:}

"Western" dietary patterns at 52y was not significantly associated with proximal colon cancer after $32 \mathrm{y} / \mathrm{u}$.

"Prudent" dietary patterns at 52y was not significantly associated with proximal colon, distal colon, or rectal cancer after $32 y$ f/u.

Results for the "western" dietary pattern were similar when men and women were analyzed separately.

Results for the "prudent" dietary pattern were similar in women; however, in men higher "prudent" diet score was

significantly associated with decreased risk of distal colon and rectal cancer.

\section{Significant:}

"Vegetarian" diet at 58y and colorectal cancer after $7.3 \mathrm{y}$ f/u:
Confounding, Study Limitations, and Summary of Findings

dietary pattern were similar in women; however, in men, higher "prudent" diet score was significantly associated with decreased risk of distal colon and rectal cancer.

\section{Key confounders accounted for:}

Sex, age, race/ethnicity, education, alcohol intake, physical activity, smoking, BMI, family 


\section{Study and}

Participant

Characteristics

Health Study-2 (AHS-

2)

United States

Analytic N: 77659

Participants were $64 \%$ female, $\sim 58$ yo ( $\geq 25 y$ o), $66 \%$

$\sim 26 \mathrm{~kg} / \mathrm{m} 2 \mathrm{BMI}, \sim 15 \%$ ever smokers, $\sim 7 \%$

currently use alcohol, $\sim 15 \%$ family history of prostate cancer, $\sim 19 \%$ no vigorous exercise

Excluded participants from ME and WI (no cancer registry), with prevalent cancers, no medical record verification, no date of cancer diagnosis, missing or invalid dietary data, age $<25 y$, missing data on age/sex, implausible energy intake $(\sim 19 \%$ of original sample)
- "Vegans"xliv: Red meat, poultry, fish; eggs; and dairy $<1$ time/mo

- "Lacto-ovo-vegetarian": Red meat, poultry, and fish $<1$ time/mo, eggs or dairy $>1$ time/mo

- "Pesco-vegetarian": Red meat or poultry <1 time/mo, fish >1 time/mo, and eggs/dairy in any amount

- "Semi-vegetarian": Red meat or poultry $>1$ time/mo, and all meats combined (including fish) $<1$ time/wk and eggs/dairy in any amount

- "Nonvegetarians": Red meat and poultry >1 time/mo and all meats combined (including fish) $>1$ time/wk, and eggs/dairy in any amount

"Vegetarians" vs. "nonvegetarians" consumed higher amounts of fruits, vegetables, avocados, non-fried potatoes, whole grains, legumes, soy foods, nuts and seeds, and was observed among vegetarians; and lower amounts of meats, dairy products, eggs, refined grains, added fats, sweets, snack foods and non-water beverages

Dietary assessment methods: >220-item, validated $F F Q$, at baseline, at $\sim 58 \mathrm{y}$

\section{Results}

- "Vegan", n=40: HR: 0.86, 95\% Cl: $0.59,1.24, p=0.42$

- "Lacto-ovo", n=147: HR: 0.83, 95\% Cl: 0.66, 1.05, $p=0.11$

- "Pesco", n=35: HR: 0.58, 95\% Cl: $0.40,0.84, p=0.004$

- "Semi", n=30: HR: 0.93, 95\% Cl: $0.62,1.38, p=0.71$

- “Nonvegetarian", n=238: HR: 1.00

Vegetarian diet at 58y and colorectal cancer after $7.3 \mathrm{y} / \mathrm{u}$ :

- “Vegetarian", n=252: HR: 0.79, 95\% Cl: 0.64, 0.97

- "Nonvegetarian", n=238: HR: 1.00

- $p=0.03$

When results were stratified by sex and race, they were no significant associations in men or black participants. However, in women and non-black vegetarians, there was a borderline significant lower risk of colorectal cancer $(p=0.08)$.

"Vegetarian" diet at 58y and rectal cancer after 7.3y f/u:

- "Vegetarian", n=55: HR: 0.66, 95\% Cl: 0.43, 1.02

- "Nonvegetarian", n=55: HR: 1.00
Confounding, Study Limitations, and

Summary of Findings

history of the cancer outcome, inflammatory

bowel disease

Other:

History of peptic ulcer, treatment for diabetes mellitus in past year, aspirin use, statin use, prior colonoscopy or flexible sigmoidoscopy, supplemental calcium use, energy intake, hormone therapy use, fiber intake

\section{Limitations:}

- Did not account for history of colorectal polyps

- Only assessed dietary intake once at baseline; did not account for possible changes in dietary intake over $\mathrm{f} / \mathrm{u}$

- Did not fully account for missing data

- No preregistered statistical plans; potential for selective outcome reporting

Funding Sources: NIH, World Cancer

Research Fund

Summary: Consuming a "vegetarian" vs. "nonvegetarian" diet at 58y was associated with a significant reduction in risk of colorectal cancer after $7.3 y \mathrm{f} / \mathrm{u}$. When results were broken down by type of "vegetarian" diet, consuming a "pescovegetarian" diet vs a "nonvegetarian" diet at 58y was associated with lower risk, while there were no

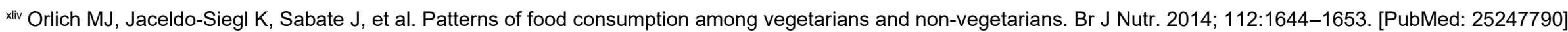




\section{Study and}

Participant

Characteristics

\section{Intervention/Exposure and Outcomes}

Outcome assessment methods: State cancer registries, patient f/u with medical record verification
Results

- $p=0.06$

\section{Non-Significant:}

"Vegetarian" vs. "nonvegetarian" diet at $58 y$ was not significantly associated with colon cancer after $7.3 \mathrm{y} / \mathrm{u}$.

\section{Park, $2017^{34}$ \\ PCS (Multiethnic Cohort study)}

\section{United States}

Analytic N: 190949

Participants were $\%$ female, $~ 60$ yo (45$75 \mathrm{y}), \sim 26 \mathrm{~kg} / \mathrm{m} 2 \mathrm{BMI}$, $\sim 70 \%$ men and $\sim 45 \%$ women were ever smokers, $\sim 15 \mathrm{~g} / \mathrm{d}$ men and $\sim 5 \mathrm{~g} / \mathrm{d}$ women alcohol

\section{Dietary patterns:}

- Healthy Eating Index 2010 (HEI-2010)xlv

- Positive components: Total vegetables, geens and beans, total fruit, whole fruit, whole grains, seafood and plant proteins, total protein foods, dairy, fatty acids

- Negative components: Refined grained, added sugars, solid fats, sodium

- Alternative Healthy Eating Index 2010 (AHEI-2010) $)^{\text {xlvi }}$

- Positive components: Vegetables (not potatoes, French fries), Fruit, Whole Grains, Long-Chain Fats (EPA + DHA), PUFA

- Neutral components: Alcohol

- Negative components: Red and Processed Meat, Sugar Sweetened

\section{Significant:}

Men, $\mathrm{HEI}-2010$ score at $60 \mathrm{y}$ and colorectal cancer after 16y f/u:

- $\quad$ Q1, $n=702, H R: 1.00$

- Q2, $\mathrm{n}=496, \mathrm{HR}: 0.81,95 \% \mathrm{Cl}$ : $0.72,0.91$

- $Q 3, n=434, H R: 0.80,95 \% \mathrm{Cl}$ : $0.70,0.91$

- $\mathrm{Q} 4, \mathrm{n}=339, \mathrm{HR}: 0.72,95 \% \mathrm{Cl}$ : $0.62,0.82$

- $\quad \mathrm{Q} 5, \mathrm{n}=267, \mathrm{HR}: 0.69,95 \% \mathrm{Cl}$ : $0.59,0.80$

- $\quad$-trend $<0.001$

Women, HEl-2010 score at 60y and colorectal cancer after $16 \mathrm{y} / \mathrm{u}$ :
Confounding, Study Limitations, and Summary of Findings

differences with "vegan", "lacto-ovo", or "semivegetarian" diets.

When results were stratified by sex and race, they were no significant associations in men or black participants. However, in women and non-black vegetarians, there was a borderline significant lower risk of colorectal cancer.

Finally, "vegetarian" diet at 58y was not significantly associated with risk of rectal or colon cancer after 7.3y f/u-, when they were analyzed separately.

\section{Key confounders accounted for:}

Sex, age, race/ethnicity, alcohol intake, physical activity, smoking, BMI, family history of the cancer outcome, colorectal polyps

Other:

Multivitamin use, NSAID use, menopausal status, hormone replacement therapy, total energy intake

\section{Limitations:}

- Did not account for socioeconomic status, inflammatory bowel disease

- Only assessed dietary intake once at baseline; did not account for possible changes in dietary intake over f/u

- Did not fully account for missing data

- No preregistered statistical plans; potential for selective outcome

xlv Guenther PM, Casavale KO, Reedy J, et al. Update of the Healthy Eating Index: HEI-2010. J Acad Nutr Diet. 2013; 113:569-580.

xivi Chiuve SE, Fung TT, Rimm EB, et al. Alternative dietary indices both strongly predict risk of chronic disease. J Nutr. 2012; 142:1009-1018. 


\section{Study and}

Participant

Characteristics

Excluded those who were not African American, Native Hawaiian, Japanese American, Latino and white, prevalent colorectal cancer, implausible dietary intake $(\sim 12 \%$ of original sample)

\section{Intervention/Exposure and Outcomes}

Beverages and Fruit Juice, Trans FA,

Sodium

- alternate Mediterranean diet score (aMED) $)^{\mathrm{xlvii}}$

- Positive components: Vegetables (not potatoes), legumes, fruits, nuts, whole grains, fish, MUFA/SFAs

- Neutral components: Alcohol

- Negative components: Red and processed meat

- Dietary Approaches to Stop Hypertension (DASH) score xlviii $^{-1}$

- Positive components: Vegetables (not potatoes), nuts and legumes, fruit and fruit juice, whole grains, low-fat dairy

- Negative components: Red and processed meat, sweetened beverages sodium

Dietary assessment methods: >180-item, validated FFQ at baseline, at $\sim 60 \mathrm{y}$

Outcome assessment methods: Surveillance, Epidemiology and End Results Program tumor registries in Hawaii and California, National Death Index

\section{Results}

Confounding, Study Limitations, and Summary of Findings

- Q1, $\mathrm{n}=279, \mathrm{HR}: 1.00$

- $Q 2, n=312, \mathrm{HR}: 0.87,95 \% \mathrm{Cl}$ : $0.74,1.03$

- Q3, n=389, HR: 0.86, 95\% Cl: $0.73,1.01$

- $\quad$ Q4, n=421, HR: $0.78,95 \% \mathrm{Cl}$ : $0.66,0.91$

- Q5, n=536, HR: $0.82,95 \% \mathrm{Cl}$ : $0.70,0.96$

- $\quad$-trend $=0.008$

Men, AHEl-2010 score at 60y and colorectal cancer after $16 \mathrm{y} / \mathrm{u}$

- Q1, n=571, HR: 1.00

- Q2, n=429, HR: $0.84,95 \% \mathrm{Cl}$ : $0.73,0.95$

- Q3, n=410, HR: $0.82,95 \% \mathrm{Cl}$ : $0.72,0.93$

- Q4, n=465, HR: 0.92, 95\% Cl: $0.81,1.04$

- Q5, n=412, HR: $0.75,95 \% \mathrm{Cl}$ : $0.65,0.85$

- $\quad$-trend<0 001 reporting

Funding Sources: NIH

Summary: In men, increased adherence to the HEI-2010, AHEI-2010, aMED, and DASH scores at 60y was significantly associated with lower risk of colorectal cancer after $16 y$ f/u

In women, increased adherence to the HEI2010 and DASH scores, but not the AHEI2010 or aMED, were significantly associated with lower risk of colorectal cancer after $16 y$ $f / u$.

Increased adherence to the HEI-2010, AHEI2010, and DASH scores were associated with significantly lower risk of left colon and rectum cancer, but not right colon cancer.

When results were stratified by race, increased adherence to dietary pattern scores was significantly associated with lower risk of colorectal cancer in all groups, except AfricanAmericans.

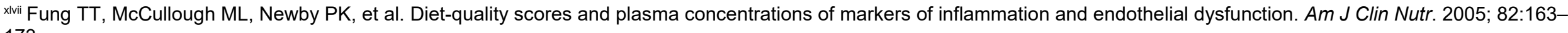
173.

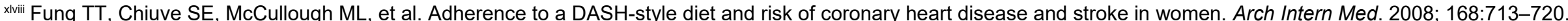




\section{Study and}

Participant

Intervention/Exposure and Outcomes

\section{Results}

Men, aMED score at 60y and colorectal

cancer after 16y f/u:

- $Q 1, n=432: H R: 1.00$

- Q2, $\mathrm{n}=405: \mathrm{HR}: 0.98,95 \% \mathrm{Cl}$ : $0.85,1.12$

- Q3, n=468: HR: $0.99,95 \% \mathrm{Cl}$ : $0.87,1.14$

- Q4, n=380: HR: $0.84,95 \% \mathrm{Cl}$ : $0.72,0.97$

- Q5, n=553: HR: $0.84,95 \% \mathrm{Cl}$ : $0.73,0.97$

- $p$-trend $=0.004$

Men, DASH score at $60 \mathrm{y}$ and colorectal cancer after 16y f/u:

- Q1, n=556: HR: 1.00

- Q2, n=328: HR: $0.85(0.74-0.97)$

- Q3, n=535: HR: 0.81 (0.72-0.92)

- $Q 4, n=340: H R: 0.82(0.71-0.95)$

- $\mathrm{Q} 5, \mathrm{n}=479$ : HR: $0.75(0.66-0.86)$

- $p$-trend<0 001

Women, DASH score at $60 \mathrm{y}$ and colorectal cancer after 16y f/u:

- Q1, n=435: HR: 1.00

- Q2, n=298: HR: 0.98 (0.85-1.14)

- $Q 3, n=469: H R: 0.86(0.75-0.99)$

- $\mathrm{Q} 4, \mathrm{n}=304$ : HR: 0.90 (0.77-1.05)
Confounding, Study Limitations, and Summary of Findings 


\section{Study and}

Participant

Characteristics

\section{Results}

Intervention/Exposure and Outcomes
- $\mathrm{Q}, \mathrm{n}=431: \mathrm{HR}: 0.86(0.75-1.00)$

- $p$-trend $=0.04$
Confounding, Study Limitations, and

Summary of Findings

Higher adherence to the HEI-2010, AHEI2010, and DASH scores were associated with significantly lower risk of left colon and rectum cancer, but not right colon cancer.

When results were stratified by race, higher adherence to the following dietary pattern scores was significantly associated with lower risk of colorectal cancer among the following race/ethnicity groups:

- African American: None

- Native Hawaiian: None (trend for HEl-2010, DASH)

- Japanese American: HEI-2010, DASH (trend for AHEI-2010)

- Latino: HEI-2010, AHEI-2010, aMED, DASH

- White: HEI-2010, AHEI-2010, DASH

\section{Non-Significant:}

Women, AHEI-2010 score at 60y was not significantly associated with colorectal cancer after $16 \mathrm{y} / \mathrm{u}$. 


\section{Study and}

Participant

Characteristics
Confounding, Study Limitations, and

Summary of Findings

Women, aMED score at 60y was not

significantly associated with colorectal cancer after $16 y \mathrm{f} / \mathrm{u}$.

\section{Petimar, 2018 ${ }^{35}$}

PCS (Nurses' Health

Study, Health

Professional Follow-

up Study)

\section{United States}

Analytic N: 78012

(NHS), 46695 (HPFS)

Participants were $63 \%$

female, $~ 55 y o$,

$\sim 25 \mathrm{~kg} / \mathrm{m} 2 \mathrm{BMI}, \sim 20 \%$

women and $\sim 10 \%$

men were current

smokers, $\sim 5 \%$ women

and $\sim 15 \%$ men $>2$

drinks/d

Exclude those with history of cancer or ulcerative colitis, without birth dates, missing diet data, implausible energy

\section{Dietary patterns:}

- $\quad$ Alternative Healthy Eating Index 2010 (AHEl-2010) $)^{\text {lix }}$

- Positive components: Vegetables (not potatoes, French fries), Fruit, Whole Grains, Long-Chain Fats (EPA + DHA), PUFA

- Neutral components: Alcohol

- Negative components: Red and Processed Meat, Sugar Sweetened Beverages and Fruit Juice, Trans FA, Sodium

- $\quad$ alternate Mediterranean diet score (aMED)'

- Positive components: Vegetables (not potatoes), legumes, fruits, nuts, whole grains, fish, MUFA/SFAs

- Neutral components: Alcohol

- Negative components: Red and processed meat

- Dietary Approaches to Stop Hypertension (DASH) scorel

- Positive components: Vegetables (not potatoes), nuts and legumes, fruit and fruit juice, whole grains, low-fat dairy

○ Negative components: Red and

\section{Significant:}

aMED score at 55y, in men and women pooled, was significantly associated with rectal cancer after $26 y$ f/u: NS

- Q1, n=120: HR: 1.00

- Q2, n=121: HR: $0.94(0.68,1.30)$

- Q3, n=118: HR: $0.84(0.64,1.09$

- Q4, n=103: HR: $0.72(0.54,0.95$

- Q5, n=103: HR: $0.76(0.54,1.07$

- $p$-trend=0.02

When women and men were analyzed separately, results were similar in men and no longer significant in women.

\section{Non-Significant:}

DASH score at 55y, in men and women pooled, was not significantly associated with colorectal cancer (colorectal, colon, distal colon, proximal colon, rectal cancer) after 26y f/u

- In women only, results were simila

- In men only, increased DASH

\section{Key confounders accounted for:}

Sex, age, alcohol intake, physical activity, smoking, BMI, family history of the cancer outcome, inflammatory bowel disease, colorectal polyps

\section{Other:}

Total energy intake, NSAID use, previous colorectal cancer screening, multivitamin use, supplemental calcium use, menopausal status, postmenopausal hormone use

\section{Limitations:}

- Did not account for race/ethnicity, socioeconomic status

- Did not fully account for missing data

- $\quad$ No preregistered statistical plans; potential for selective outcome reporting

Funding Sources: NIH

Summary: Higher aMED adherence at 55y was significantly associated with lower risk of rectal cancer after $26 y$ f/u. When women and men were analyzed separately, results were similar in men, and no longer significant in women. aMED score was not significantly

xix Chiuve SE, Fung TT, Rimm EB, et al. Alternative dietary indices both strongly predict risk of chronic disease. J Nutr. 2012; 142:1009-1018.

' Fung TT, McCullough ML, Newby PK, et al. Diet-quality scores and plasma concentrations of markers of inflammation and endothelial dysfunction. Am J Clin Nutr. 2005; 82:163-173.

li Fung TT, Chiuve SE, McCullough ML, et al. Adherence to a DASH-style diet and risk of coronary heart disease and stroke in women. Arch Intern Med. 2008; 168:713-720. 


\section{Study and \\ Participant \\ Characteristics \\ intake ( $30 \%$ of \\ original sample) \\ Intervention/Exposure and Outcomes

processed meat, sweetened beverages, \\ sodium}

Results

Dietary assessment methods: Validated, FFQ at baseline and every $4 \mathrm{y}$ after, baseline at 55y

Outcome assessment methods: Follow-up with participantes, medical record review,

National Death Index, next of kin

Roswall, 2015
PCS (Women's
Lifestyle and Health
cohort)

\section{Dietary patterns:}

Healthy Nordic food index (HNFI)

- Positive components: Cabbage, root vegetables, apples and pears, rye bread, oatmeal, fish adherence was significantly

associated with lower total colorectal, total colon, and distal colon cancer risk

aMED score at 55y, in men and women pooled, was not significantly associated with colorectal cancer (colorectal, colon, distal colon, proximal colon) after $26 \mathrm{y} f / \mathrm{u}$

- In women only, results were similar

- In men only, increased aMED adherence was significantly associated with lower total colorectal cancer risk

AHEl-2010 score at 55y, in men and women pooled, was not significantly associated with colorectal cancer (colorectal, colon, distal colon, proximal colon, rectal cancer) after $26 y \mathrm{f} / \mathrm{u}$

- In women only, results were similar

- In men only, increased AHEI-2010 adherence was significantly associated with lower total colorectal colon cancer risk

\section{Significant: N/A}

\section{Non-Significant:}

HNFI score at 39y (categorical and continuous) was not significantly associated with colorectal cancer.
Confounding, Study Limitations, and Summary of Findings

associated with colorectal cancer (colorectal, colon, distal colon, proximal colon), which the exception of in men, increased aMED adherence was significantly associated with lower total colorectal cancer risk.

DASH score at 55y was not significantly associated with colorectal cancer (colorectal, colon, distal colon, proximal colon, rectal cancer) after $26 y \mathrm{f} / \mathrm{u}$, which the exception that in men, increased DASH adherence was significantly associated with lower total colorectal, total colon, and distal colon cancer risk.

AHEI-2010 score at 55y, in men and women pooled, was not significantly associated with colorectal cancer (colorectal, colon, distal colon, proximal colon, rectal cancer) after 26y $f / u$, with the exception that in men, increased AHEI-2010 adherence was significantly associated with lower total colorectal colon cancer risk

Key confounders accounted for:

Sex, education, alcohol intake, smoking, BMI,

Other:

Oral contraceptives, energy intake, red and processed meat

\section{Limitations:}




\section{Study and}

Participant

Characteristics

\section{Sweden}

Analytic N: 45222

Participants were $100 \%$ female, $39 y$, $23 \mathrm{~kg} / \mathrm{m} 2 \mathrm{BMI}, 41 \%$ never smokers, 2.9 $\mathrm{g} / \mathrm{d}$ alcohol

Exclude those who emigrated before $\mathrm{f} / \mathrm{u}$, extreme energy intakes, missing covariate data, prevalent colorectal cancer $(\sim 8 \%$ of original sample)

\section{Schulpen, $2020^{44}$ \\ Nested Case-Control Study (Netherlands \\ Cohort Study (NLCS)) \\ The Netherlands}

Analytic N: 8050
Intervention/Exposure and Outcomes

Dietary assessment methods: 80 -item,

validated FFQ at baseline, 39y

\section{Outcome assessment methods: Swedish}

Cancer Registry
Confounding, Study Limitations, and Summary of Findings
- Did not account for age, race/ethnicity, physical activity, family history of the cancer outcome, inflammatory bowel disease, colorectal polyps

- Only assessed dietary intake once at baseline; did not account for possible changes in dietary intake over $\mathrm{f} / \mathrm{u}$

- Did not fully account for missing data

- No preregistered statistical plans; potential for selective outcome reporting

Funding Sources: Swedish Research Council

Summary: Healthy Nordic Food Index score at $39 y$ was not significantly associated with risk of colorectak cancer during f/u.

\section{Dietary patterns:}

- alternate Mediterranean diet scores (aMED) $)^{\text {lii }}$, and without alcohol (aMEDr)

- Positive components: Vegetables (not potatoes), legumes, fruits, nuts, whole grains, fish, MUFA/SFAs

- Neutral components: Alcohol

- Negative components: Red and processed meat

\section{Significant:}

In women, smoking status significantly modified the relationship between aMEDr (per 2-pt increment) and colorectal cancer:

- Never smokers, $n=256$ : HR: 1.00, $95 \% \mathrm{Cl}: 0.88,1.13$

- Former smokers, n=350: HR: 0.78, Daily energy intake $95 \% \mathrm{Cl}: 0.63,0.98$

- Current smokers, n=309: HR: 1.21, - Did not account for race/ethnicity, 95\% Cl: 0.96, 1.51

\section{Key confounders accounted for:}

Sex, age, education, alcohol intake, physical activity, smoking, BMI, family history of the cancer, lung disease

Other: inflammatory bowel disease, colorectal

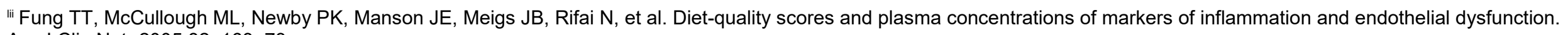
Am J Clin Nutr 2005;82: 163-73. 


\section{Results}

Confounding, Study Limitations, and Summary of Findings

\section{Study and}

Participant

Characteristics

Participants were $52 \%$ female, $\sim 61$ yo (55$69 \mathrm{y}), \sim 25 \mathrm{~kg} / \mathrm{m}^{2} \mathrm{BMI}$, $\sim 55 \%$ former-smokers; $\sim 10 \mathrm{~g} / \mathrm{d}$ alcohol

Excluded those with prevalent cancer (except skin); missing data on diet and alcohol $(\sim 10 \%$ of the original sample)
Intervention/Exposure and Outcomes

- WCRF/AICR score ${ }^{\text {liii }}$, diet only, and without alcohol

- Positive components: Vegetables and fruit, dietary fiber

- Negative components: Red and processed meat, sugary drinks, alcohol, sodium, energy-dense foods

Dietary assessment methods: 150-item, validated FFQ at baseline, age $\sim 61 \mathrm{y}$

\section{Outcome assessment methods:}

Netherlands Cancer Registry and the nationwide Dutch Pathology Registry, and review of pathology records

\section{Non-Significant}

Adherence to the aMED, with and without alcohol, and the WCRF/AICR, diet only score, with and without alcohol, at 61y was not significantly associated with risk of colorectal, colon, proximal, distal, or rectal cancer in men or women after 20.3y f/u.

Results were also not significant when stratified by smoking status in men, alcohol consumption, body mass index, education, or family history of colorectal cancer. polyps

- Only assessed dietary intake once at baseline; did not account for possible changes in dietary intake over f/u

- Did not account for missing data

- $\quad$ No preregistered statistical plans; serious potential for selective outcome reporting

Funding Sources: Wereld Kanker Onderzoek Fonds Nederland, World Cancer Research Fund International

Summary: aMED and WCRF/AICR scores, with and without alcohol, at 61y were not significantly associated with risk colorectal cancer after 20.3y f/u.

Results were also not significant when stratified by smoking status in men, alcohol consumption, body mass index, education, or family history of colorectal cancer. However, in female former smokers, greater adherence to the aMEDr was significantly associated with lower risk of colorectal cancer.

\section{Shin, 2018 37 \\ PCS (Japan Public Health Center-based Prospective Study}

\section{Dietary patterns:}

Adherence to 3 dietary patterns (categorical quintiles) identified via principal component analysis:

\section{Significant:}

In men, "prudent" dietary pattern score at

$57 y$ and colorectal cancer

\section{Key confounders accounted for:}

Sex, age, race/ethnicity, physical activity, smoking
- Q1, n=318: HR: 1.00

Other:

- Q2, n=299: HR: $0.95,95 \% \mathrm{Cl}$ :

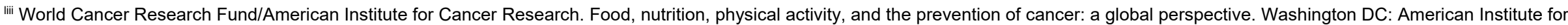
Cancer Research; 2007. 


\section{Study and}

Participant

Characteristics

\section{Japan}

Analytic N: 93,062

Participants were 53\%

female, $\sim 57$ yo, 23 $\mathrm{kg} / \mathrm{m} 2 \mathrm{BMI}, \sim 36 \%$ past or current smokers, and $\sim 46 \%$ ever drinkers

Excluded those with history of cancer, from one study center missing outcome data, missing diet data, unsuitable energy intake, non-Japanese ethnicity, inaccurate birth date, moved out of area, who died, lost to $\mathrm{f} / \mathrm{u}(30 \%$ of original sample)
- "Prudent" dietary pattern: higher loadings for high intakes of vegetables, fruit, noodle, potatoes, soy products, mushroom, seaweed

- "Westernized" dietary pattern: higher loadings for meat and processed meat, eel, dairy foods, fruit juice, coffee, tea, soft beverages, sauces, alcohol

- "Traditional" dietary pattern: higher loadings for pickles, seafood, fish (oily-, salty-, leanfish, and salmon), chicken, sake

Dietary assessment methods: 138-item validated FFQ at baseline, 57y

\section{Outcome assessment methods: Cancer} registry system and local medical records

\section{Results}

Confounding, Study Limitations, and Summary of Findings

\section{$0.81,1.11$}

- Q3, n=274: HR: $0.82,95 \% \mathrm{Cl}$ : $0.70,0.96$

- Q4, n=308: HR: 0.90, 95\% Cl: $0.77,1.05$

- Q5, $\mathrm{n}=315: \mathrm{HR}: 0.85,95 \% \mathrm{Cl}$ : $0.72,1.00$

- $\quad$-trend $=0.0346$

Results were similar for distal colon cancer.

In women, "prudent" dietary pattern score at $57 y$ and colorectal cancer

- Q1, n=50: HR: 1.00

- Q2, n=45: HR: 0.93, 95\% Cl: 0.62, 1.39

- Q3, $\mathrm{n}=45: \mathrm{HR}: 0.94,95 \% \mathrm{Cl}: 0.62$, 1.41

- Q4, n=46: HR: $0.96,95 \% \mathrm{Cl}: 0.63$, 1.44

- Q5, $n=73: H R: 1.53,95 \% \mathrm{Cl}: 1.04$, 2.23

- $\quad$-trend $=0.0463$

\section{Total energy intake}

\section{Limitations:}

- Did not account for socioeconomic status, alcohol intake, anthropometry, family history of the cancer outcome, inflammatory bowel disease, colorectal polyps

- Only assessed dietary intake once at baseline; did not account for possible changes in dietary intake over $\mathrm{f} / \mathrm{u}$

- Did not fully account for missing data

- No preregistered statistical plans; potential for selective outcome reporting

Funding Sources: National Cancer Center Research and Development Fund (Japan), Ministry of Health, Labour and Welfare of Japan

Summary: Higher adherence to the "prudent" dietary pattern was significantly associated with decreased risk of colorectal cancer and distal cancer in men and increased risk of rectal cancer in women. Higher adherence to the "westernized" dietary pattern was significantly associated with increased risk of colon cancer and distal cancer in women.

In women, "westernized" dietary pattern score at 57y and colon cancer:

- Q1, n=147: HR: 1.00

- Q2, n=149: HR: $1.15,95 \% \mathrm{Cl}$ : 


\section{Study and}

Participant

Characteristics
Intervention/Exposure and Outcomes
Results

Confounding, Study Limitations, and

Summary of Findings

\section{$0.91,1.45$}

- Q3, $\mathrm{n}=165: \mathrm{HR}: 1.40,95 \% \mathrm{Cl}$ : $1.12,1.76$

- Q4, n=131: HR: 1.24, 95\% Cl: $0.97,1.59$

- Q5, $\mathrm{n}=117$ : HR: $1.28,95 \% \mathrm{Cl}$ : $0.98,1.68$

- $\quad \mathrm{p}$-trend $=0.0337$

Results were similar for distal colon cancer.

\section{Non-Significant:}

"Prudent" dietary pattern score at $57 y$ was not significantly associated with colon, proximal colon, or rectal cancer in men, or colorectal, colon cancer, proximal colon, or distal colon cancer in women.

"Westernized" dietary pattern score at 57y was not significantly associated with colorectal, colon, proximal colon, distal colon, or rectal cancer in men, or colorectal, proximal colon, or rectal cancer in women.

"Traditional" dietary pattern score at 57y was not significantly associated with colorectal, colon, proximal colon, distal colon, or rectal cancer in both men and women. 


\section{Study and}

Participant

Characteristics

Tabung, 2018a ${ }^{38}$

PCS (Nurses' Health

Study, Health

Professional Follow-

up Study)

\section{United States}

Analytic N: 121,050

Participants were 61\% female, had a mean age of $\sim 62 \mathrm{y}, \sim 25$ $\mathrm{kg} / \mathrm{m} 2 \mathrm{BMI}, \sim 60 \%$ drinkers, $\sim 5$ drinks/wk, and $\sim 8 \%$ current smokers.

Excluded those with history of cancer. incomplete dietary data, implausible energy intake $(30 \%$ of original sample)

\section{Dietary patterns:}

- Empirical Dietary Inflammatory Pattern (EDIP) Score (categorical - quintiles)

- Higher in processed meat, red meat, organ meat, fish (other than dark-meat fish), other vegetables, refined grains, high-energy beverages, low-energy beverages, tomatoes

- Lower in beer, wine, tea, coffee, dark yellow vegetables, green leafy vegetables, snacks, fruit juice, pizza

Dietary assessment methods: Validated, FFQ at baseline and every $4 \mathrm{y}$ after, baseline at 55y

Outcome assessment methods: Follow-up with participantes, medical record review,

National Death Index, next of kin
Results

\section{Significant:}

In men and women combined, EDIP scores at 55y and colorectal cancer after 26y f/u:

- Q1, n=113: HR: 1.00

- Q2, n=121: HR: $1.02,95 \% \mathrm{Cl}$ : $0.90,1.16$

- Q3, n=140: HR: $1.13,95 \% \mathrm{Cl}$ : 1.00, 1.28

- Q4, n=130: HR: $1.17,95 \% \mathrm{CI}$ : $1.03,1.32$

- $\quad$ Q5, $n=151:$ HR: $1.32,95 \% \mathrm{Cl}$ : $1.12,1.55$

- $\quad$-trend<0.001

Results were similar men, women, and in combined analyses for colon, distal colon, and proximal colon. Results were also significant, for men only, with rectal cancer.

When stratified by BMI < or $>25 \mathrm{~kg} / \mathrm{m}^{2}$, results were significant for men in both groups, and only in women with $\mathrm{BMI}<25 \mathrm{~kg} / \mathrm{m}^{2}$.

When results were stratified by alcohol intake (no drink, 0.1-1 drink/d, >1 drink/d), results were significant for men consuming no drinks or 0.1-1 drink/d, and in women consuming no drinks.
Confounding, Study Limitations, and Summary of Findings

Key confounders accounted for:

Sex, age, race/ethnicity, alcohol intake, physical activity, anthropometry, smoking, family history of the cancer outcome

\section{Other:}

Total energy intake, multivitamin use, history of endoscopy

\section{Limitations:}

- Did not account for socioeconomic status, inflammatory bowel disease, colorectal polyps

- Did not fully account for missing data

- No preregistered statistical plans; potential for selective outcome reporting

Funding Sources: $\mathrm{NCl}, \mathrm{NIH}$, Dana-Farber Harvard Cancer Center, and Stand Up to Cancer grant

Summary: Higher adherence to EDIP scores over up to 26 y f/u (most proinflammatory diet) was significantly associated with risk of colorectal cancer, colon cancer, proximal colon cancer, distal colon cancer in men, women, and both combined, as well as riak of rectal cancer in men. Results were not significant for rectal cancer risk in women or men/women combined.

When stratified by $B M I<$ or $>25 \mathrm{~kg} / \mathrm{m}^{2}$, results were significant for men in both groups, and only in women with $B M l<25 \mathrm{~kg} / \mathrm{m}^{2}$. When stratified by alcohol intake (no drink, 0.1-1 


\section{Study and}

Participant

Characteristics

Intervention/Exposure and Outcomes

\section{Tabung, 2018b 39 \\ PCS (Nurses' Health Study, Health \\ Professional Follow- up Study)}

\section{United States}

Analytic N: 120,401

Participants were $62 \%$ female with a mean age $\sim 64 \mathrm{y}, \sim 25 \mathrm{~kg} / \mathrm{m} 2$ BMI, $~ 60 \%$ drinkers, and $\sim 8 \%$ current smokers.

\section{Excluded those with} history of cancer or ulcerative colitic, incomplete dietary data, implausible energy intake $(30 \%$ of original sample)

\section{Dietary patterns:}

- Empirical dietary index for hyperinsulinemia (EDIH)

- Higher in red meat, processed meat, low energy beverages, cream soups, margarine, poultry, high energy beverages, butter, French fries, other fish, low fat dairy, eggs, tomatoes, cruciferous vegetables

- Lower in wine, coffee, high fat dairy, green leafy vegetables, whole fruits, dark yellow vegetables, snacks

Dietary assessment methods: Validated, FFQ at baseline and every $4 y$ after, baseline at 55y

Outcome assessment methods: Follow-up with participantes, medical record review, National Death Index, next of kin
Results

\section{Non-Significant:}

EDIP scores at 55y was not significantly associated with rectal cancer after $26 \mathrm{y} f / u$, in women or pooled analyses.

Confounding, Study Limitations, and Summary of Findings

drink/d, $>1$ drink/d), results were significant for men consuming no drinks or 0.1-1 drink/d, and in women consuming no drinks.

\section{Key confounders accounted for:}

Significant:

In men and women combined, EDIH score at $55 \mathrm{y}$ and colorectal cancer after 26y f/u:

- $\mathrm{Q} 1, \mathrm{n}=552: \mathrm{HR} \cdot 1.00$

- Q2, n=525: HR: $0.97,95 \% \mathrm{Cl}$ : $0.86,1.10$

- Q3, n=566: HR: $1.10,95 \% \mathrm{Cl}$ $0.97,1.24$

- Q4, n=529: HR: $1.10,95 \% \mathrm{Cl}$ $0.97,1.25$

- Q5, n=511: HR: $1.26,95 \% \mathrm{Cl}$ : $1.12,1.42$

- $p$-trend $<0.0001$

Results were similar for men, women, and men and women combined for colorectal, colon, and distal colon cancer. Results were also significantly for women and men and women combined for proximal colon cancer, and in men for rectal cancer.

When stratified by BMl $<$ or $>25 \mathrm{~kg} / \mathrm{m}^{2}$, results were significant for men in both groups, and only in women with $\mathrm{BMl}>25 \mathrm{~kg} / \mathrm{m}^{2}$.
Sex, age, race/ethnicity, alcohol intake, physical activity, anthropometry, smoking, family history of the cancer outcome

Other:

Total energy intake, multivitamin use, history of endoscopy

\section{Limitations:}

- Did not account for socioeconomic status, inflammatory bowel disease

- Did not fully account for missing data

- No preregistered statistical plans; potential for selective outcome reporting

Funding Sources: $\mathrm{NCl}, \mathrm{NIH}$, Dana-Farber Harvard Cancer Center, and Stand Up to Cancer grant

Summary: Higher EDIH scores at 55y was significantly associated with higher risk of colorectal cancer after $26 y$ f/u. Results were similar for men, women, and men and women combined for colorectal, colon, and distal colon cancer. Results were also significantly for women and men and women combined for proximal colon cancer, and in men for rectal cancer. 


\section{Study and}

Participant
Characteristics

Intervention/Exposure and Outcomes

Characteristics

\section{PCS (NIH)- \\ AARP) Diet and Health Study) \\ United States}

Analytic N: 398458

Participants were $41 \%$ female, majority $>60 \mathrm{y}$ $(50-71 \mathrm{y}), \sim 65 \%$ overweight or obese, $37 \%$ never smokers

\section{- Healthy Eating Index 2010 (HEl-2010) $)^{\text {liv }}$}

- Positive components: Total vegetables, geens and beans, total fruit, whole fruit, whole grains, seafood and plant proteins, total protein foods, dairy, fatty acids

- Negative components: Refined grained, added sugars, solid fats, sodium

- Positive components: Vegetables, legumes, fruit, cereals, fish, MUFA+PUFA/SFA

- Neutral components: Alcohol

- Negative components: Meat, dairy products
- Mediterranean diet score ${ }^{\mathrm{lv}}$

\section{Results}

When results were stratified by physical activity (MET-h/wk below/above median), results were significant for men and women below the median.

\section{Non-Significant:}

Higher EDIH scores over up to $26 \mathrm{y} f / \mathrm{u}$ were not significantly associated with proximal colon cancer in men, or in rectal cancer in women or men and women combined.

Significant:

In normal weight men, mediterranean diet score at $60 \mathrm{y}$ and colorectal cancer

Confounding, Study Limitations, and Summary of Findings

Higher EDIH scores over up to 26 y f/u were not significantly associated with proximal colon cancer in men, or in rectal cancer in women or men and women combined.

When stratified by $B M I<$ or $>25 \mathrm{~kg} / \mathrm{m}^{2}$, results were significant for men in both groups, and only in women with $B M 1>25 \mathrm{~kg} / \mathrm{m}^{2}$. When results were stratified by physical activity (MET-h/wk below/above median), results were significant for men and women below the median.

\section{Key confounders accounted for:}

activity, smoking, BMI, family history of the ( $n=4483$ cases) after $123 \mathrm{mo} f / u$ :

- Q1: HR: 1.00

- Q2: HR: $0.79,95 \%$ Cl: $0.66,0.96$

- Q3: HR: $0.66,95 \% \mathrm{Cl}: 0.54,0.82$

- Q4: HR: $0.67,95 \%$ Cl: 0.54, 0.84

- Q5: HR: $0.65,95 \% \mathrm{Cl}: 0.51,0.83$

- $p$-trend $=0.0004$

Results were also significant in overweight $(p=0.0013)$ and obese $(p=0.0508)$ men cancer outcome, colorectal polyps

\section{Other:}

\section{Energy intake}

\section{Limitations:}

- Did not account for alcohol intake, inflammatory bowel disease

- Excluded subjects with history of with colon cancer, and underweight

- Only assessed dietary intake once at baseline; did not account for possible changes in dietary intake over $\mathrm{f} / \mathrm{u}$ colorectal polyps, first-degree relatives

liv Guenther PM, Casavale KO, Reedy J, et al. Update of the Healthy Eating Index: HEl-2010. J Acad Nutr Diet. 2013; 113:569-580.

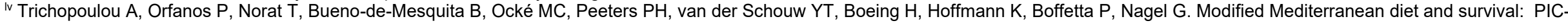
elderly prospective cohort study. Bmj. 2005 Apr 28;330(7498):991. 


\section{Study and \\ Participant \\ Characteristics \\ Excluded those with history of cancer or end-stage renal disease, history of colorectal polyps, first- degree relatives with colon cancer, underweight, missing height or weight \\ Intervention/Exposure and Outcomes \\ - Dietary Approaches to Stop Hypertension (DASH) score ${ }^{\text {lvi }}$ \\ - Positive components: Vegetables (not potatoes), nuts and legumes, fruit and fruit juice, whole grains, low-fat dairy \\ - Negative components: Red and processed meat, sweetened beverages, sodium} implausible energy intake, questionnaires completed by proxy ( $30 \%$ original sample)
Dietary assessment methods: 124 -item validated FFQ at baseline, $60 y$

Outcome assessment methods: State cancer registries

\section{Results}

In normal weight men, $\mathrm{HEI}-2010$ score at $60 y$ and colorectal cancer ( $n=4483$ cases) after $123 \mathrm{mo} / \mathrm{u}$ :

- Q1: HR: 1.00

- Q2: HR: $0.94,95 \% \mathrm{Cl}: 0.77,1.14$

- Q3: HR: 0.83, 95\% Cl: 0.67, 1.03

- Q4: HR: $0.73,95 \% \mathrm{Cl}: 0.58,0.91$

- Q5: HR: 0.67, 95\% Cl: 0.54, 0.84

- $\quad p$-trend $=0.0001$

Results were also significant in overweight $(p<0.0001)$ and obese $(p=0.0394)$ men

In normal weight men, DASH score at $60 \mathrm{y}$ and colorectal cancer ( $n=4483$ cases) after $123 \mathrm{mo} / \mathrm{u}$ :

- Q1: HR: 1.00

- Q2: HR: 0.91, 95\% Cl: 0.08, 1.11

- Q3: HR: 0.79, 95\% Cl: 0.64, 0.99

- Q4: HR: $0.83,95 \% \mathrm{Cl}: 0.66,1.04$

- Q5: HR: 0.67, 95\% Cl: 0.54, 0.84

- $\quad$-trend $=0.0005$
Confounding, Study Limitations, and Summary of Findings

- Did not account for missing data

- No preregistered statistical plans; serious potential for selective outcome reporting

Funding Sources: $\mathrm{NIH}$

Summary: In men, higher Mediterranean, HEI2010, and DASH scores were significantly associated with lower risk of colorectal cancer after $123 m o$ f/u.

In women, higher HEl-2010 score was significantly associated with lower risk of colorectal cancer in overweight women.

Higher DASH score was significantly associated with lower risk of colorectal cancer in normal weight and obese women.

Mediterranean diet score was not significantly associated with risk of colorectal cancer in women.

Results were also significant in overweight men $(p<0.0001)$, but not in obese men $(p=0.0801)$

Ivi Fung TT, Chiuve SE, McCullough ML, et al. Adherence to a DASH-style diet and risk of coronary heart disease and stroke in women. Arch Intern Med. 2008; 168:713-720. 


\section{Study and}

Participant

Characteristics
Results

Intervention/Exposure and Outcomes
When probablities were examined, in normal weight, overweight, and obese men, higher scores on all 3 dietary

patterns was associated with decreased risk of colorectal cancer.

In overweight women, $\mathrm{HEI}-2010$ score at $60 \mathrm{y}$ and colorectal cancer ( $\mathrm{n}=2032$ cases) after $123 \mathrm{mo} / \mathrm{u}$ :

- Q1: HR: 1.00

- Q2: HR: 0.87, 95\% Cl: 0.65, 1.16

- Q3: HR: $0.94,95 \% \mathrm{Cl}: 0.71,1.25$

- Q4: HR: $0.73,95 \% \mathrm{Cl}: 0.55,0.98$

- Q5: HR: $0.64,95 \% \mathrm{Cl}: 0.47,0.86$

- $p$-trend $=0.0001$

Results were not significant in normal weight $(p=0.1557)$ and obese $(p=0.0573)$ women

In normal weight women, DASH score at $60 \mathrm{y}$ and colorectal cancer $(\mathrm{n}=2032$ cases) after $123 \mathrm{mo} / \mathrm{u}$ :

- Q1: HR: 1.00

- Q2: HR: $0.86,95 \% \mathrm{Cl}: 0.68,1.09$

- Q3: HR: $0.70,95 \%$ Cl: $0.53,0.93$

- Q4: HR: $0.86,95 \%$ Cl: $0.66,1.13$

- Q5: HR: $0.73,95 \% \mathrm{Cl}: 0.56,0.95$
Confounding, Study Limitations, and Summary of Findings 


\section{Study and}

Participant

Characteristics

\section{Results}

- $p$-trend $=0.0005$

Results were also significant in obese women $(p=0.0128)$, but not in overweight women $(p=0.1256)$

When probablities were examined, in normal weight women, higher DASH score was associated with decreased risk of colorectal cancer. In overweight and obese women, increased $\mathrm{HEI}-2010$ and DASH scores were associated with decreased risk of colorectal cancer.

\section{Non-Significant:}

In normal weight, overweight, and obese women, mediterranean diet score at $60 \mathrm{y}$ was not significantly associated with colorectal cancer ( $n=2032$ cases) after $123 \mathrm{mo} / \mathrm{u}$.

Vargas, 201641
PCS (Women's
Health Initiative
Observational Study)
United States

Analytic N: 78273
- Healthy Eating Index 2010 (HEl-2010) $)^{\mid v i i}$

- Positive components: Total vegetables, geens and beans, total fruit, whole fruit, whole grains, seafood and plant proteins, total protein foods, dairy, fatty acids

- Negative components: Refined grained,

\section{Dietary patterns:} added sugars, solid fats, sodium

\section{Significant:}

HEI-2010 score at 63y and colorectal cancer after 12.4 y f/u:

- Q1, n=209: HR: 1.00

- Q2, n=189: HR: 0.88, 95\% Cl: 0.72 , 1.08

- $Q 3, n=175: H R: 0.81,95 \% \mathrm{Cl}: 0.66$, 0.99
Confounding, Study Limitations, and

Summary of Findings 


\section{Study and}

Participant

Characteristics

Participants were $100 \%$ female, $\sim 63 y$, $\sim 27 \mathrm{~kg} / \mathrm{m} 2$, 19\% never smokers, $\sim 2.5$ alcohol servings/wk

Excluded those with history of cancer, colorectal diagnosis during first year of $f / u$, missing f/u or dietary data, implausible energy intakes $(\sim 16 \%$ of original sample)

\section{Intervention/Exposure and Outcomes}

- $\quad$ Alternative Healthy Eating Index 2010

(AHEl-2010) $)^{\text {lviii }}$

- Positive components: Vegetables (not potatoes, French fries), Fruit, Whole Grains, Long-Chain Fats (EPA + DHA), PUFA

- Neutral components: Alcohol

- Negative components: Red and Processed Meat, Sugar Sweetened Beverages and Fruit Juice, Trans FA, Sodium

- $\quad$ alternate Mediterranean diet score (aMED) $)^{\text {lix }}$

- Positive components: Vegetables (not potatoes), legumes, fruits, nuts, whole grains, fish, MUFA/SFAs

- Neutral components: Alcohol

- Negative components: Red and processed meat

- Dietary Approaches to Stop Hypertension (DASH) score ${ }^{\mathrm{lx}}$

- Positive components: Vegetables (not potatoes), nuts and legumes, fruit and fruit juice, whole grains, low-fat dairy

- Negative components: Red and processed meat, sweetened beverages sodium

\section{Results}

- $Q 4, n=172: H R: 0.77,95 \% \mathrm{Cl}: 0.63$, 0.95

- $Q 5, n=166: H R: 0.73,95 \% \mathrm{Cl}: 0.59$, 0.90

- $\quad \mathrm{p}$-trend $=0.032$

Higher HEI-2010 score was significantly associated with decreased riusk of colon cancer, but not rectal cancer.

DASH score at 63y and colorectal cancer after $12.4 \mathrm{y}$ f/u:

- Q1, n=195: HR: 1.00

- Q2, n=177: HR: 0.84, 95\% Cl: 0.68, 1.03

- Q3, $n=193: H R: 0.72,95 \% \mathrm{Cl}: 0.59$, 0.89

- Q4, n=183: HR: $0.74,95 \% \mathrm{Cl}: 0.60$, 0.91

- Q5, n=163: HR: 0.78, 95\% Cl: 0.62, 0.97

- $\quad$-trend $=0.021$

Higher DASH score was significantly associated with decreased riusk of colon cancer, but not rectal cancer.
Confounding, Study Limitations, and Summary of Findings

- Only assessed dietary intake once at baseline; did not account for possible changes in dietary intake over $\mathrm{f} / \mathrm{u}$

- Did not fully account for missing data

- No preregistered statistical plans; potential for selective outcome reporting

\section{Funding Sources: NIH}

Summary: Higher HEI-2010 and DASH scores at $63 y$ were significantly associated with decreased risk of colorectal and colon cancer after $12.4 y$ f/u, but not rectal cancer.

AHEI and aMED scores were not significantly associated with risk of colorectal, colon, or rectal cancer after $12.4 \mathrm{y} / \mathrm{u}$.

Iviii Chiuve SE, Fung TT, Rimm EB, et al. Alternative dietary indices both strongly predict risk of chronic disease. J Nutr. 2012; 142:1009-1018.

lix Fung TT, McCullough ML, Newby PK, et al. Diet-quality scores and plasma concentrations of markers of inflammation and endothelial dysfunction. Am J Clin Nutr. 2005; 82:163-173.

${ }^{1 x}$ Fung TT, Chiuve SE, McCullough ML, et al. Adherence to a DASH-style diet and risk of coronary heart disease and stroke in women. Arch Intern Med. 2008; 168:713-720. 


\section{Study and}

Participant

Characteristics
Results
Confounding, Study Limitations, and

Summary of Findings
Dietary assessment methods: 122-item validated $F F Q$, at baseline, $\sim 63 y$

Outcome assessment methods: Participant f/u, medical records, pathology reports

\section{Non-Significant:}

AHEI score at $63 y$ was not significantly associated with risk of colorectal, colon, and rectal cancer after $12.4 \mathrm{y} / \mathrm{u}$ $(p=0.427)$.

aMED score at $63 y$ was not significantly associated with risk of colorectal, colon, and rectal cancer after $12.4 \mathrm{y} / \mathrm{u}$

$(p=0.217)$

\section{Voortman, 2017 \\ PCS (Rotterdam Study)}

\section{Dietary patterns:}

The Netherlands

Analytic N: 9627

Participants were $58 \%$ female, 64.1yo,

$26.3 \mathrm{~kg} / \mathrm{m} 2,32 \%$ never smokers, $61 \%<10 \mathrm{~g} / \mathrm{d}$ alcohol

\section{Excluded those} without reliable dietary data, prevalent cancer cases, missing outcome data $(\sim 19 \%$ of original sample)
- Dutch Dietary Guidelines 2015 score, continuous

- Positive components: Vegetables, legumes, fruit, nuts, whole grains, fish, dairy products, unsaturated fats and oils, tea

Dutch Dietary Guidelines 2015 score and colorectal cancer $(n=324)$ after $11 \mathrm{y} f / \mathrm{u}: \mathrm{HR}$ $0.90,95 \%$ Cl: $0.84,0.96), p<0.05$

\section{Non-Significant: N/A}

- Negative components: Replace refined grains with whole-grain products, red meat, processed meat, alcohol, sodium

Dietary assessment methods: 170 to 389 item, validated FFQ at baseline, $\sim 64 \mathrm{y}$

Outcome assessment methods: Nationwide registry of histopathology and cytopathology, f/u with general practitioners

\section{Significant:}

\section{Key confounders accounted for:}

Sex, age, race/ethnicity, education, employment status, alcohol intake, physical activity, smoking, BMI

\section{Other:}

Cohort, total energy intake

\section{Limitations:}

- Did not account for race/ethnicity, family history of the cancer outcome, inflammatory bowel disease, colorectal polyps

- Only assessed dietary intake once at baseline; did not account for possible changes in dietary intake over f/u

- Did not fully account for missing data

- No preregistered statistical plans; potential for selective outcome reporting 


\section{Study and}

Participant

Characteristics
Confounding, Study Limitations, and Summary of Findings

Funding Sources: Erasmus University Medical Center and Erasmus University Rotterdam; the Netherlands Organization for Health Research and Development; the Research Institute for Diseases in the Elderly; the Netherlands Genomics Initiative; the Ministry of Education, Culture and Science; the Ministry of Health, Welfare and Sports; the European Commission (DG XII); and the Municipality of Rotterdam

Summary: Higher Dutch Dietary Guidelines 2015 score at $64 y$ was significantly associated with lower risk of colorectal cancer after 11y f/u.

\section{Vulcan, $2019^{42}$}

PCS (Malmö Diet and Cancer Study)

\section{Sweden}

Analytic N: 27931

Participants were $\sim 60 \%$ female, $\sim 59$ yo, $26.5 \mathrm{~kg} / \mathrm{m} 2 \mathrm{BMl}, 37 \%$ never smokers, $\sim 72 \%$ drnak 0-20g/d alcohol

Excluded those with a history of cancer $(\sim 1 \%)$

\section{Dietary patterns:}

- Colorectal Diet Quality Index (CDQI)

- Positive components: Fiber, dairy products

- Negative components: Processed meat

Dietary assessment methods: 168-item FFQ, and a 7-day menu diet history book at baseline, $\sim 59 \mathrm{y}$

Outcome assessment methods: Swedish Cancer Registry

\section{Significant:}

CDQI score at $59 y$ and colorectal cancer $(n=923)$ after 502136 person years $\mathrm{f} / \mathrm{u}$ :

- $0-3, n=135:$ HR: 1.00

- 4-6, n=222: HR: 0.77, 95\% Cl: 0.65, 0.91

- 7-9, n=187: HR: 0.74, 95\% Cl: 0.61, 0.89

- $10-12, n=46$ : HR: $0.57,95 \% \mathrm{Cl}: 0.43$, 0.75

- $\quad$-trend $<0.001$

Results were similar for colon and rectal cancer, and when women and men were analyzed separately.

\section{Key confounders accounted for:}

Sex, age, education, alcohol intake, physical activity, smoking, BMI

\section{Other:}

Diet assessment method, season, total energy

\section{Limitations:}

- Did not account for race/ethnicity, anthropometry, family history of the cancer outcome, inflammatory bowel disease, colorectal polyps

- Only assessed dietary intake once at baseline; did not account for possible changes in dietary intake over $\mathrm{f} / \mathrm{u}$

- No preregistered statistical plans; potential for selective outcome reporting 


\section{Study and}

Participant

Intervention/Exposure and Outcomes

Characteristics
Results

Non-Significant: N/A
Confounding, Study Limitations, and Summary of Findings

Funding Sources: Malmö Hospital

Foundation for Cancer Prevention, South

Region of healthcare

Summary: Higher colorectal diet quality index score at 59y was significantly associated with lower risk of colorectal, colon, and rectal cancer after $f / u$. 
Table 6. Summary of the results from studies that examined the relationship between dietary patterns and colorectal cancerixi

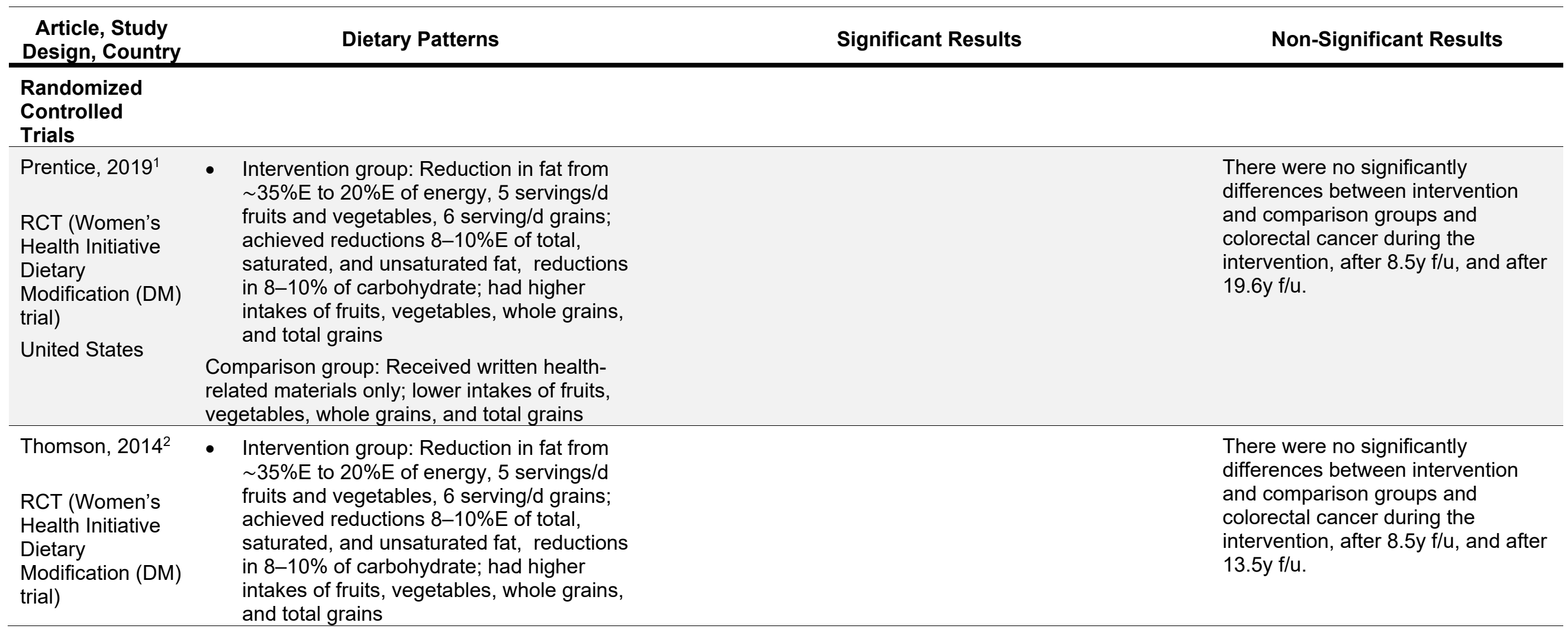

Ixi Abbreviations: AHEI-2010, Alternative Healthy Eating Index-2010; aMED, Alternative Mediterranean Diet Score ; BMI, body mass index; CDQI, Colorectal Diet Quality Index; d, day; DASH, Dietary Approaches to Stop Hypertension; DFA, "High-dairy", "high-fruit-and-vegetable", "low, alcohol" dietary pattern; DM, dietary modification; DP, Dietary pattern; \%E, \% of energy; EDIH, Empirical dietary index for hyperinsulinemia; EDIP, Empirical dietary inflammatory pattern; EPIC, European Prospective Investigation into Cancer and Nutrition; FFQ, food frequency questionnaire; FSAm-NPS, Nutrient Profiling System of the British Food Standards Agency (modified version); f/u, follow-up; HNFI, Healthy Nordic food index; HR, hazard ratio; IMI, Italian Mediterranean Index; MEDI-LITE, Mediterranean diet score; MD, Mediterranean diet; MDS, Mediterranean Diet Score; mo, month or months; N/A, Not applicable; NIH, National Institutes of Health; NOVA, Ultra-processed food score; NS, Not significant; NR, Not reported; PCS, prospective cohort study; pt, point; RCT, randomized controlled trial; SEER, Surveillance, Epidemiology, and End Results Program; WCRF/AICR, World Cancer Research Fund/American Institute for Cancer Research; wk, week(s); y, year(s) 


\begin{tabular}{|c|c|c|c|}
\hline $\begin{array}{l}\text { Article, Study } \\
\text { Design, Country }\end{array}$ & Dietary Patterns & Significant Results & Non-Significant Results \\
\hline United States & $\begin{array}{l}\text { Comparison group: Received written } \\
\text { health-related materials only; lower intakes } \\
\text { of fruits, vegetables, whole grains, and } \\
\text { total grains }\end{array}$ & & \\
\hline \multicolumn{4}{|l|}{$\begin{array}{l}\text { Obervational } \\
\text { Studies }\end{array}$} \\
\hline $\begin{array}{l}\text { Boden, } 2019^{27} \\
\text { PCS } \\
\text { (Vasterbotten } \\
\text { Intervention } \\
\text { Programme) }\end{array}$ & \multirow{2}{*}{$\begin{array}{l}\text { Mediterranean diet score (MDS) } \\
\text { - } \quad \text { Positive components: Vegetables and } \\
\text { potatoes, fruit and fresh juices, wholegrain } \\
\text { cereals, fish and fish products, } \\
\text { MUFA+PUFA/SFA, } \\
\text { - } \quad \text { Moderation components: Alcohol } \\
\text { - } \quad \text { Negative components: Meat and meat } \\
\text { products, dairy products }\end{array}$} & & \multirow[t]{2}{*}{$\begin{array}{l}\text { Mediterranean diet score at } 46 y \\
\text { was not significantly associated with } \\
\text { colorectal cancer }(n=1036) \text { after } \\
15 y \text { f/u in all participants, or when } \\
\text { men and women were analyzed } \\
\text { separately. }\end{array}$} \\
\hline Sweden & & & \\
\hline \multirow{3}{*}{$\begin{array}{l}\text { Cheng, } 2018^{43} \\
\text { PCS (lowa } \\
\text { Women's Health } \\
\text { Study) } \\
\text { United States }\end{array}$} & \multirow{2}{*}{$\begin{array}{l}\text { Evolutionary-concordance score } \\
\text { Positive components: Vegetables, fruits, } \\
\text { lean meats, fish, nuts, fruit and vegetable } \\
\text { diversity, calcium } \\
\text { - Negative components: Red and processed } \\
\text { meats, sodium, dairy foods, grains and } \\
\text { starches, baked goods, sugar-sweetened } \\
\text { beverages, alcohol }\end{array}$} & & \multirow[t]{2}{*}{$\begin{array}{l}\text { Evolutionary-concordance score at } \\
61 y \text { (categorical and continuous) } \\
\text { was not significantly associated with } \\
\text { colorectal cancer ( } n=1731 \text { after 18y } \\
\text { f/u). }\end{array}$} \\
\hline & & & \\
\hline & \multicolumn{2}{|l|}{$\begin{array}{l}\text { Mediterranean diet score } \\
\text { - Positive components: Vegetables (not } \\
\text { potatoes), legumes, fruits, nuts, whole } \\
\text { grains, fish, MUFA/SFAs } \\
\text { - Neutral components: Alcohol } \\
\text { - Negative components: Red and processed } \\
\text { meat }\end{array}$} & $\begin{array}{l}\text { Mediterranean diet score at } 61 \mathrm{y} \\
\text { (categorical and continuous) was } \\
\text { not significantly associated with } \\
\text { colorectal cancer ( } n=1731 \text { ) after } \\
18 \mathrm{y} \text { f/u. }\end{array}$ \\
\hline $\begin{array}{l}\text { Deschasaux, } \\
2018^{4} \\
\text { PCS (European }\end{array}$ & $\begin{array}{l}\text { Nutrient Profiling System of the British Food } \\
\text { Standards Agency (modified version) (FSAm- } \\
\text { NPS) score }\end{array}$ & $\begin{array}{l}\text { FSAm-NPS score at } 51 \mathrm{y} \text { and risk of colorectal cancer after } \\
\text { 15.3y f/u: } \\
\text { - Q1, n=1144: HR: } 1.00\end{array}$ & \\
\hline
\end{tabular}




\begin{tabular}{|c|c|c|c|}
\hline $\begin{array}{l}\text { Article, Study } \\
\text { Design, Country }\end{array}$ & Dietary Patterns & Significant Results & Non-Significant Results \\
\hline $\begin{array}{l}\text { Prospective } \\
\text { Investigation into } \\
\text { Cancer and } \\
\text { Nutrition (EPIC)) }\end{array}$ & $\begin{array}{l}\text { Overall diet score assigned based on } \\
\text { energy, sugar, saturated fatty acid, } \\
\text { sodium, fibers, proteins, and } \\
\text { fruits/veqetables/lequmes/nuts. }\end{array}$ & $\begin{array}{l}\text { - Q2, n=1150: HR: } 1.07,95 \% \text { Cl: 0.99, } 1.17 \\
\text { - Q3, n=1152: HR: } 1.07,95 \% \text { Cl: } 0.98,1.17 \\
\text { - Q4, n=1195: HR: } 1.12,95 \% \text { Cl: } 1.02,1.22\end{array}$ & \\
\hline $\begin{array}{l}\text { Denmark, } \\
\text { France, } \\
\text { Germany, } \\
\text { Greece, Italy, the } \\
\text { Netherlands, } \\
\text { Norway, Spain, } \\
\text { Sweden, UK }\end{array}$ & $\begin{array}{l}\text { Higher FSAm-NPS score had higher } \\
\text { intakes of alcohol, energy and red and } \\
\text { processed meat, lower intakes of dietary } \\
\text { fibers, vegetables, fruit, fish, and lean meat }\end{array}$ & $\begin{array}{l}\text { - Q5, } n=1165: \text { HR: } 1.11,95 \% \mathrm{Cl}: 1.01,1.22 \\
\text { - } \mathrm{p} \text {-trend= } 0.02 \\
\text { Continuous, per } 2 \mathrm{pt} \text { increment, } \mathrm{n}=5086 \text { cases: HR: } 1.03 \text {, } \\
95 \% \mathrm{Cl}: 1.00,1.06, \mathrm{p} \text {-trend }=0.03\end{array}$ & \\
\hline $\begin{array}{l}\text { Fasanelli, } 2017^{28} \\
\text { PCS (European } \\
\text { Prospective } \\
\text { Investigation into } \\
\text { Cancer and } \\
\text { Nutrition) } \\
\text { Italy }\end{array}$ & $\begin{array}{l}\text { Italian Mediterranean Index (IMI) (mediation } \\
\text { with waist-to-hip ratio) } \\
\text { - Positive components: Pasta, } \\
\text { Mediterranean vegetables (raw tomatoes, } \\
\text { cooked leafy vegetables, raw leafy } \\
\text { vegetables, onion or garlic, mixed salad or } \\
\text { mixed vegetables), fruits, legumes, olive } \\
\text { oil, fish } \\
\text { - Neutral components: Alcohol } \\
\text { - Negative components: Soft drinks, butter, } \\
\text { red meat, potatoes }\end{array}$ & 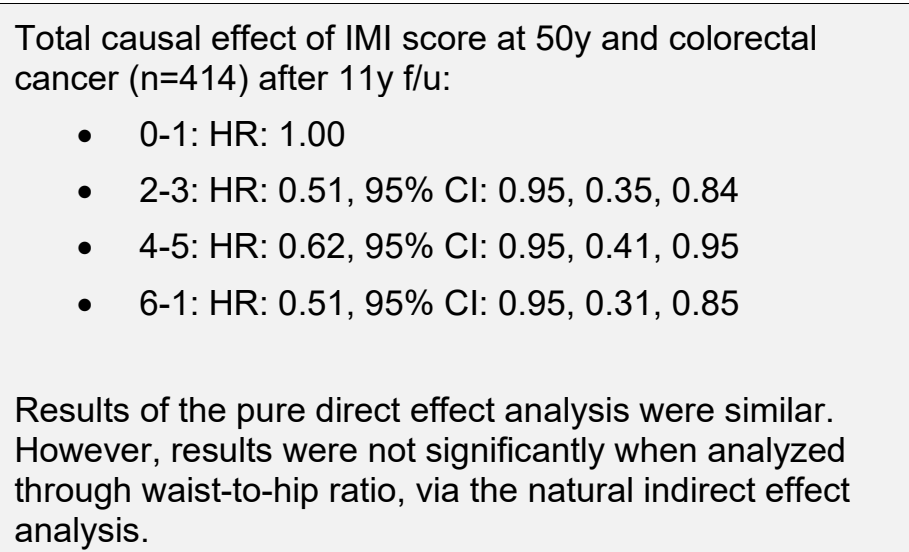 & \\
\hline Fiolet, $2018^{7}$ & $\begin{array}{l}\text { Ultra-processed food score (NOVA), } \\
\text { categorical (quartiles) }\end{array}$ & & $\begin{array}{l}\text { Ultra-processed food score at } 49 \mathrm{y} \\
\text { and colorectal cancer after } 5.4 \mathrm{y} / \mathrm{u} \text { : }\end{array}$ \\
\hline $\begin{array}{l}\text { PCS (NutriNet- } \\
\text { Santé) } \\
\text { France }\end{array}$ & $\begin{array}{l}\text { Main food groups contributing to NOVA } \\
\text { score were sugary drinks, drinks, starchy } \\
\text { foods and breakfast cereals, ultra- } \\
\text { processed fruits and vegetables, dairy } \\
\text { products, meats, fish, and eggs, processed } \\
\text { meats, fats, and salty snacks }\end{array}$ & & $\begin{array}{l}\text { Q1, n=48: HR: } 1.00 \\
\text { Q2, n=43: HR: } 1.12,95 \% \\
\text { Cl: } 0.74,1.70 \\
\text { Q3, n=36: HR: } 1.22,95 \% \\
\text { Cl: } 0.79,1.89 \\
\text { Q4, n=26: HR: } 1.23,95 \% \\
\text { Cl: } 1.08,1.40 \\
\text { - p-trend=0.07 }\end{array}$ \\
\hline
\end{tabular}




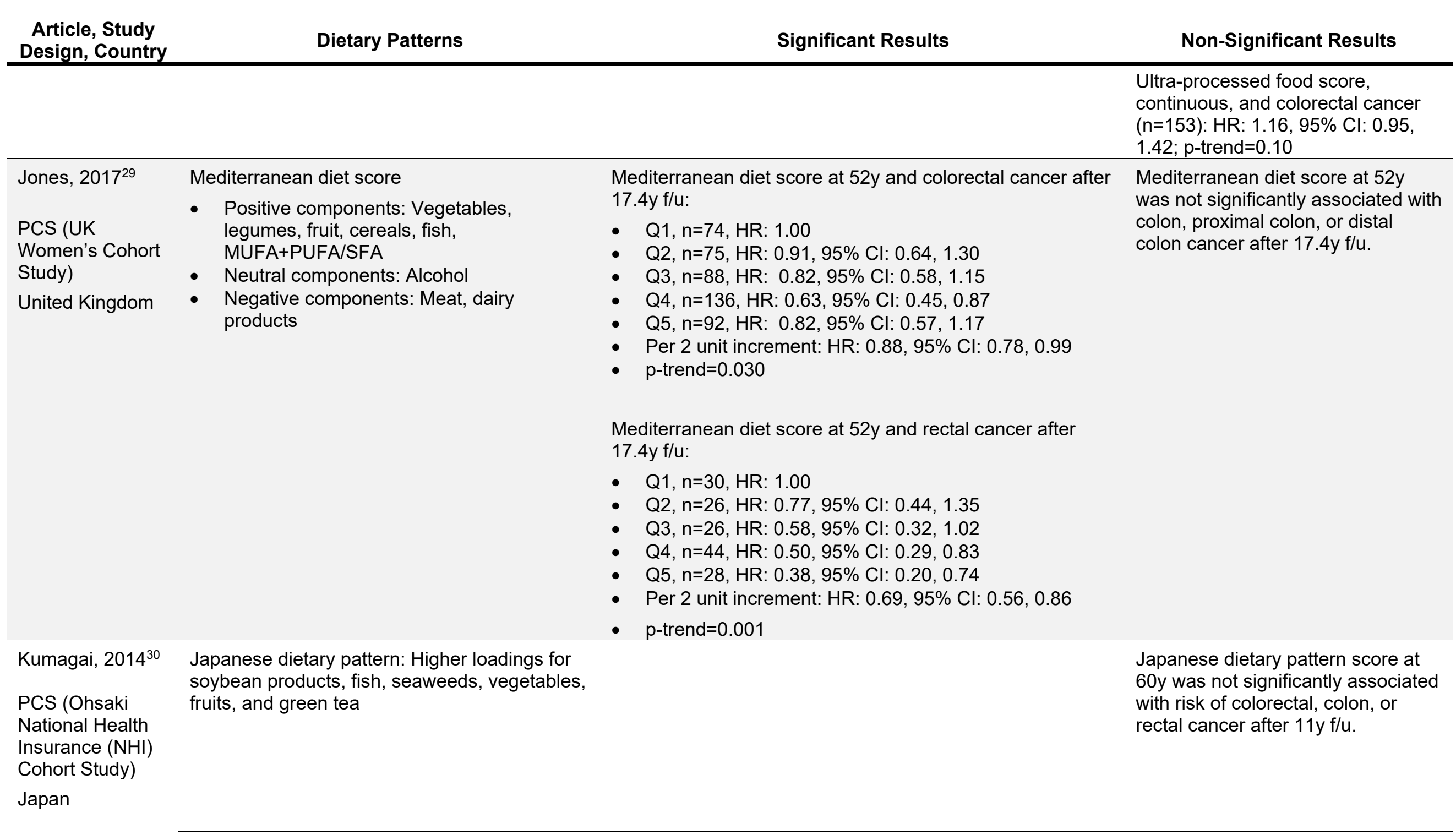




\begin{tabular}{|c|c|c|c|}
\hline $\begin{array}{l}\text { Article, Study } \\
\text { Design, Country }\end{array}$ & Dietary Patterns & Significant Results & Non-Significant Results \\
\hline & $\begin{array}{l}\text { Animal food dietary pattern: Higher loadings for } \\
\text { beef pork, ham, sausage, chicken, liver, butter, } \\
\text { coffee, and alcoholic beverages }\end{array}$ & & $\begin{array}{l}\text { Animal food dietary pattern score at } \\
60 \mathrm{y} \text { was not significantly associated } \\
\text { with risk of colorectal, colon, or } \\
\text { rectal cancer after } 11 \mathrm{y} / \mathrm{u} \text {. }\end{array}$ \\
\hline & $\begin{array}{l}\text { High-dairy, high-fruit-and-vegetable, low, } \\
\text { alcohol (DFA) dietary pattern: Higher loadings } \\
\text { for dairy products (milk and yoghurt), } \\
\text { margarine, fruits, and vegetables (carrot, } \\
\text { pumpkin and tomato), and lower for rice, miso } \\
\text { soup, and alcoholic beverages }\end{array}$ & 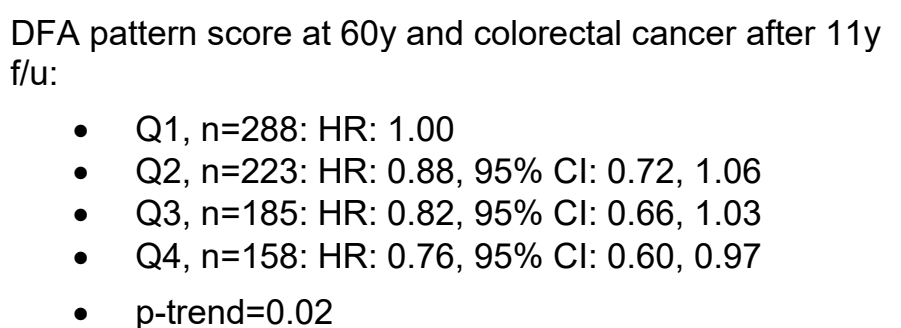 & $\begin{array}{l}\text { DFA dietary pattern score at } 60 \mathrm{y} \\
\text { was not significantly associated with } \\
\text { risk of colon cancer after } 11 \mathrm{y} / \mathrm{u} \text {. }\end{array}$ \\
\hline
\end{tabular}

DFA pattern score at $60 \mathrm{y}$ and rectal cancer after $11 \mathrm{y} f / \mathrm{u}$ :

- Q1, $n=127: H R: 1.00$

- Q2, n=82: HR: $0.79,95 \% \mathrm{Cl}: 0.58,1.07$

- Q3, n=68: HR: $0.76,95 \%$ Cl: 0.53, 1.08

- Q4, n=46: HR: $0.56,95 \% \mathrm{Cl}: 0.37,0.84$

- $p$-trend $=0.0003$

\section{Lavalette, $2018^{16} \quad$ Alternate Healthy Eating Index 2010 (AHEI- \\ PCS (NutriNet- Sante study) \\ France \\ 2010) \\ - Positive components: Vegetables (not potatoes, French fries), Fruit, Whole Grains, Long-Chain Fats (EPA + DHA), PUFA \\ - Neutral components: Alcohol \\ - Negative components: Red and Processed Meat, Sugar Sweetened Beverages and Fruit Juice, Trans FA, Sodium}

Mediterranean diet score (MEDI-LITE)

- Positive components: Vegetables, legumes, fruit and nuts, cereals, fish, olive
AHEI-2010 score at $\sim 49 y$ (categorical and continuous) was not significantly associated with colorectal cancer after $8.5 \mathrm{y} / \mathrm{u}$.
MEDI-LITE score at $\sim 49 \mathrm{y}$ (categorical and continuous) was not significantly associated with colorectal cancer after $8.5 \mathrm{y} / \mathrm{u}$. 


\begin{tabular}{|c|c|c|c|}
\hline $\begin{array}{c}\text { Article, Study } \\
\text { Design, Country }\end{array}$ & Dietary Patterns & Significant Results & Non-Significant Results \\
\hline & $\begin{array}{ll} & \text { oil } \\
\text { - Neutral components: Alcohol } \\
\text { - Negative components: Meat, dairy } \\
\text { products }\end{array}$ & & \\
\hline & $\begin{array}{l}\text { French National Nutrition Health Program- } \\
\text { Guideline Score (PNNS-GS) } \\
\text { - Positive components: Vegetables and } \\
\text { Fruit, Seafood, Vegetable Fat } \\
\text { - Neutral components: Breads, cereals, } \\
\text { potatoes, legumes, meat and poultry, } \\
\text { seafood, and eggs, milk and dairy } \\
\text { products, alcohol } \\
\text { - Negative components: Sweetened foods, } \\
\text { soda, added fat, salt }\end{array}$ & $\begin{array}{l}\text { Higher PNNS-GS score at } \sim 49 \mathrm{y} \text { (continuous) and } \\
\text { colorectal cancer after } 8.5 \mathrm{y} \text { f/u: } \mathrm{HR}: 0.89,95 \% \mathrm{Cl}: 0.80 \text {, } \\
1.00, p=0.04\end{array}$ & $\begin{array}{l}\text { PNNS-GS score at } \sim 49 \mathrm{y} \\
\text { (categorical) was not associated } \\
\text { with risk of colorectal cancer after } \\
8.5 \mathrm{y} / \mathrm{u} \text {. }\end{array}$ \\
\hline $\begin{array}{l}\text { Liu, } 2017^{31} \\
\text { PCS (Nurses' } \\
\text { Health Study, } \\
\text { Health } \\
\text { Professional } \\
\text { Follow-up Study) } \\
\text { United States }\end{array}$ & $\begin{array}{l}\text { Empirical dietary inflammatory pattern (EDIP) } \\
\text { score (response variables: IL6, CRP (C- } \\
\text { reactive protein): Higher in red and processed } \\
\text { meats, refined grains, carbonated beverages, } \\
\text { and some vegetables; lower in beer, wine, } \\
\text { coffee, tea, yellow and leafy vegetables, and } \\
\text { fruit juice }\end{array}$ & $\begin{array}{l}\text { EDIP score at } 52 y \text { and colorectal cancer after 2,998,258 } \\
\text { person-y f/u: } \\
\text { - Q1: HR: } 1.00 \\
\text { - Q2: HR: } 0.86,95 \% \mathrm{Cl}: 0.75,0.98 \\
\text { - Q3: HR: } 0.93,95 \% \mathrm{Cl}: 0.82,1.06 \\
\text { - Q4: HR: } 0.99,95 \% \mathrm{Cl}: 0.87,1.13 \\
\text { - Q5: HR: } 1.14,95 \% \mathrm{Cl}: 0.99,1.30 \\
\text { - p-trend }=0.02\end{array}$ & \\
\hline $\begin{array}{l}\text { Mehta, } 2017^{32} \\
\text { PCS (Nurses' } \\
\text { Health Study, } \\
\text { Health } \\
\text { Professional } \\
\text { Follow-up Study) }\end{array}$ & $\begin{array}{l}\text { Western dietary pattern: Higher loadings for } \\
\text { red and processed meats, high-fat dairy } \\
\text { products (such as whole milk and cream), } \\
\text { refined grains, and desserts }\end{array}$ & $\begin{array}{l}\text { Western dietary patterns at } 52 \mathrm{y} \text { and colorectal cancer after } \\
\text { 32y f/u: } \\
\text { - Q1, } \mathrm{n}=835: \mathrm{HR}: 1.00 \\
\text { - Q2, } \mathrm{n}=822: \mathrm{HR}: 1.09,95 \% \mathrm{Cl}: 0.99,1.20 \\
\text { - Q3, } \mathrm{n}=784: \mathrm{HR}: 1.11,95 \% \mathrm{Cl}: 1.00,1.24 \\
\text { - } \mathrm{p} \text {-trend=<0.0001 }\end{array}$ & $\begin{array}{l}\text { Western dietary patterns at } 52 \mathrm{y} \text { was } \\
\text { not significantly associated with } \\
\text { proximal colon cancer after } 32 \mathrm{y} \text { f/u. } \\
\text { Results for the western dietary } \\
\text { pattern were similar when men and } \\
\text { women were analyzed separately. }\end{array}$ \\
\hline
\end{tabular}




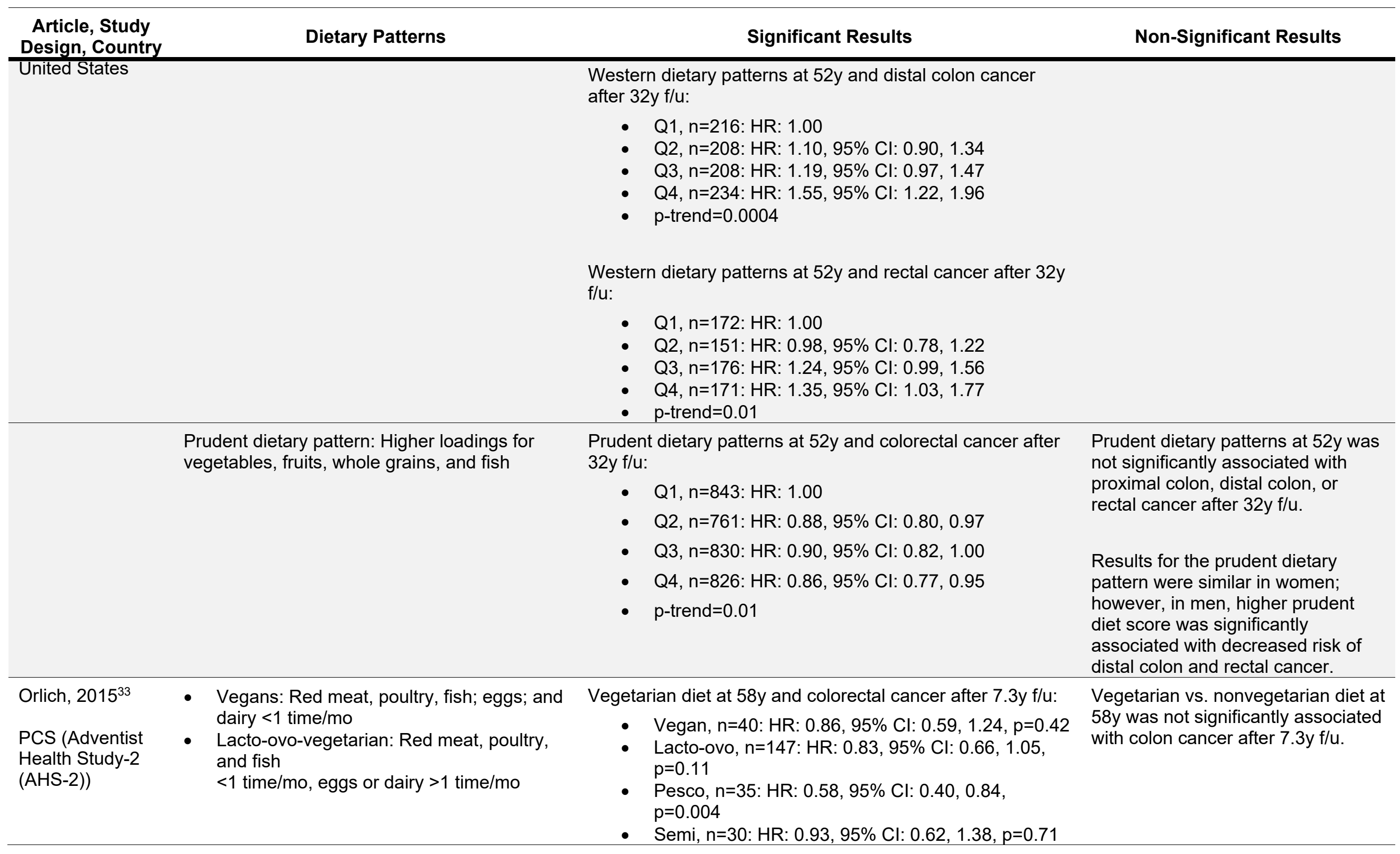




\begin{tabular}{|c|c|c|c|}
\hline $\begin{array}{c}\text { Article, Study } \\
\text { Design, Country }\end{array}$ & Dietary Patterns & Significant Results & Non-Significant Results \\
\hline United States & $\begin{array}{l}\text { Pesco-vegetarian: Red meat or poultry }<1 \\
\text { time/mo, fish }>1 \text { time/mo, and eggs/dairy in } \\
\text { any amount } \\
\text { - Semi-vegetarian: Red meat or poultry }>1 \\
\text { time/mo, and all meats combined } \\
\text { (including fish) }<1 \text { time/wk and eggs/dairy } \\
\text { in any amount } \\
\text { - Nonvegetarians: Red meat and poultry }>1 \\
\text { time/mo and all meats combined (including } \\
\text { fish) }>1 \text { time/wk, and eggs/dairy in any } \\
\text { amount } \\
\text { Vegetarians vs. nonvegetarians consumed } \\
\text { higher amounts of fruits, vegetables, avocados, } \\
\text { non-fried potatoes, whole grains, legumes, soy } \\
\text { foods, nuts and seeds, and was observed } \\
\text { among vegetarians; and lower amounts of } \\
\text { meats, dairy products, eggs, refined grains, } \\
\text { added fats, sweets, snack foods and non-water } \\
\text { beverages }\end{array}$ & $\begin{array}{l}\text { - Nonvegetarian, } \mathrm{n}=238: \mathrm{HR}: 1.00 \\
\text { Vegetarian diet at 58y and colorectal cancer after } 7.3 \mathrm{y} \mathrm{f} / \mathrm{u} \text { : } \\
\text { - Vegetarian, } \mathrm{n}=252: \mathrm{HR}: 0.79,95 \% \mathrm{Cl}: 0.64,0.97 \\
\text { - } \quad \mathrm{p}=0.03 \\
\text { When results were stratified by sex and race, they were no } \\
\text { significant associations in men or Black participants. } \\
\text { However, in women and non-black vegetarians, there was } \\
\text { a borderline significant lower risk of colorectal cancer } \\
\text { (p=0.08). } \\
\text { Vegetarian diet at } 58 \mathrm{y} \text { and rectal cancer after } 7.3 \mathrm{y} / \mathrm{f} / \mathrm{u} \\
\text { - Vegetarian, } \mathrm{n}=55: \mathrm{HR}: 0.66,95 \% \mathrm{Cl}: 0.43,1.02 \\
\text { - Nonvegetarian, } \mathrm{n}=55: \mathrm{HR}: 1.00 \\
\text { - } \mathrm{p}=0.06\end{array}$ & \\
\hline $\begin{array}{l}\text { Park, } 2017^{34} \\
\text { PCS (Multiethnic } \\
\text { Cohort study) } \\
\text { United States }\end{array}$ & $\begin{array}{l}\text { Healthy Eating Index (HEI)-2010 } \\
\text { Positive components: Total vegetables, } \\
\text { geens and beans, total fruit, whole fruit, } \\
\text { whole grains, seafood and plant proteins, } \\
\text { total protein foods, dairy, fatty acids } \\
\text { - Negative components: Refined grained, } \\
\text { added sugars, solid fats, sodium }\end{array}$ & $\begin{array}{l}\text { Men, HEI-2010 score at } 60 y \text { and colorectal cancer after } \\
\text { 16y f/u: } \\
\text { - Q1, n=702, HR: } 1.00 \\
\text { - Q2, n=496, HR: } 0.81,95 \% \mathrm{Cl}: 0.72,0.91 \\
\text { - } \mathrm{Q} 3, \mathrm{n}=434, \mathrm{HR}: 0.80,95 \% \mathrm{Cl}: 0.70,0.91 \\
\text { - } \mathrm{Q} 4, \mathrm{n}=339, \mathrm{HR}: 0.72,95 \% \mathrm{Cl}: 0.62,0.82 \\
\text { - } \mathrm{Q} 5, \mathrm{n}=267, \mathrm{HR}: 0.69,95 \% \mathrm{Cl}: 0.59,0.80 \\
\text { - } \mathrm{p} \text {-trend<0.001 } \\
\text { Women, HEI-2010 score at } 60 \mathrm{y} \text { and colorectal cancer after } \\
\text { 16y f/u: }\end{array}$ & \\
\hline
\end{tabular}




\begin{tabular}{|c|c|c|c|}
\hline $\begin{array}{c}\text { Article, Study } \\
\text { Design, Country }\end{array}$ & Dietary Patterns & Significant Results & Non-Significant Results \\
\hline & & $\begin{array}{l}\text { - Q1, n=279, HR: } 1.00 \\
\text { - } \mathrm{Q} 2, \mathrm{n}=312, \mathrm{HR}: 0.87,95 \% \mathrm{Cl}: 0.74,1.03 \\
\text { - } \mathrm{Q} 3, \mathrm{n}=389, \mathrm{HR}: 0.86,95 \% \mathrm{Cl}: 0.73,1.01 \\
\text { - } \mathrm{Q} 5, \mathrm{n}=536, \mathrm{HR}: 0.78,95 \% \mathrm{Cl}: 0.66,0.91 \\
\text { - } \mathrm{p} \text {-trend=0.008 } \\
\text { Increased adherence to the HEI-2010 was associated with } \\
\text { significantly lower risk of left colon and rectum cancer, but } \\
\text { not right colon cancer. } \\
\text { When results were stratified by race, higher HEI-2010 } \\
\text { score was significantly associated with lower risk of } \\
\text { colorectal cancer among the following race/ethnicity } \\
\text { groups: } \\
\text { - African American: None } \\
\text { - Native Hawaiian: Trend } \\
\text { - Japanese American } \\
\text { - Latino }\end{array}$ & \\
\hline & $\begin{array}{l}\text { Alternative Healthy Eating Index (AHEI)-2010 } \\
\text { - Positive components: Vegetables (not } \\
\text { potatoes, French fries), Fruit, Whole } \\
\text { Grains, Long-Chain Fats (EPA + DHA), } \\
\text { PUFA } \\
\text { - Neutral components: Alcohol } \\
\text { - Negative components: Red and Processed } \\
\text { Meat, Sugar Sweetened Beverages and } \\
\text { Fruit Juice, Trans FA, Sodium }\end{array}$ & $\begin{array}{l}\text { Men, AHEI-2010 score at } 60 y \text { and colorectal cancer after } \\
\text { 16y f/u: } \\
\text { - } \mathrm{Q} 1, \mathrm{n}=571, \mathrm{HR}: 1.00 \\
\text { - } \mathrm{Q} 2, \mathrm{n}=429, \mathrm{HR}: 0.84,95 \% \mathrm{Cl}: 0.73,0.95 \\
\text { - } \mathrm{Q} 3, \mathrm{n}=410, \mathrm{HR}: 0.82,95 \% \mathrm{Cl}: 0.72,0.93 \\
\text { - } \mathrm{Q} 4, \mathrm{n}=465, \mathrm{HR}: 0.92,95 \% \mathrm{Cl}: 0.81,1.04 \\
\text { - } \mathrm{Q} 5, \mathrm{n}=412, \mathrm{HR}: 0.75,95 \% \mathrm{Cl}: 0.65,0.85 \\
\text { - } \mathrm{p} \text {-trend<0.001 }\end{array}$ & $\begin{array}{l}\text { Women, AHEI-2010 score at } 60 \mathrm{y} \\
\text { was not significantly associated with } \\
\text { colorectal cancer after } 16 \mathrm{f} / \mathrm{u} \text {. }\end{array}$ \\
\hline
\end{tabular}




\begin{tabular}{|c|c|c|c|}
\hline $\begin{array}{c}\text { Article, Study } \\
\text { Design, Country }\end{array}$ & Dietary Patterns & Significant Results & Non-Significant Results \\
\hline & & $\begin{array}{l}\text { Increased adherence to the AHEI-2010 was associated } \\
\text { with significantly lower risk of left colon and rectum cancer, } \\
\text { but not right colon cancer. }\end{array}$ & \\
\hline & & $\begin{array}{l}\text { When results were stratified by race, increased adherence } \\
\text { to the AHEI-2010 was significantly associated with lower } \\
\text { risk of colorectal cancer among the following race/ethnicity } \\
\text { groups: } \\
\text { - African American: None } \\
\text { - Native Hawaiian: None } \\
\text { - Japanese American: Trend } \\
\text { - Latino } \\
\text { - White }\end{array}$ & \\
\hline & $\begin{array}{l}\text { Alternative Mediterranean Diet Score (aMED) } \\
\text { - Positive components: Vegetables (not } \\
\text { potatoes), legumes, fruits, nuts, whole } \\
\text { grains, fish, MUFA/SFAs } \\
\text { - Neutral components: Alcohol } \\
\text { - } \quad \begin{array}{l}\text { Negative components: Red and processed } \\
\text { meat }\end{array}\end{array}$ & $\begin{array}{l}\text { Men, aMED score at } 60 y \text { and colorectal cancer after } 16 y \\
\text { f/u: } \\
\text { - Q1, n=432: HR: } 1.00 \\
\text { - Q2, n=405: HR: } 0.98,95 \% \mathrm{Cl}: 0.85,1.12 \\
\text { - Q3, n=468: HR: } 0.99,95 \% \mathrm{Cl}: 0.87,1.14 \\
\text { - Q4, n=380: HR: } 0.84,95 \% \mathrm{Cl}: 0.72,0.97 \\
\text { - Q5, } \mathrm{n}=553: \text { HR: } 0.84,95 \% \mathrm{Cl}: 0.73,0.97 \\
\text { - } \mathrm{p} \text {-trend=0.004 }\end{array}$ & $\begin{array}{l}\text { Women, aMED score at } 60 \mathrm{y} \text { was } \\
\text { not significantly associated with } \\
\text { colorectal cancer after } 16 \mathrm{y} \text { f/u. } \\
\text { aMED score was not significantly } \\
\text { associated with left colon, right } \\
\text { colon, or rectum cancer. }\end{array}$ \\
\hline & & $\begin{array}{l}\text { When results were stratified by race, aMED adherence was } \\
\text { not significantly associated with lower risk of colorectal } \\
\text { cancer any race/ethnicity groups. }\end{array}$ & \\
\hline & $\begin{array}{l}\text { DASH Score } \\
\text { - Positive components: Vegetables (not } \\
\text { potatoes), nuts and legumes, fruit and fruit } \\
\text { juice, whole grains, low-fat dairy }\end{array}$ & $\begin{array}{l}\text { Men, DASH score at } 60 \mathrm{y} \text { and colorectal cancer after } 16 \mathrm{y} \\
\text { f/u: } \\
\text { - Q1, n=556: HR: } 1.00 \\
\text { - Q2, n=328: HR: } 0.85(0.74-0.97)\end{array}$ & \\
\hline
\end{tabular}




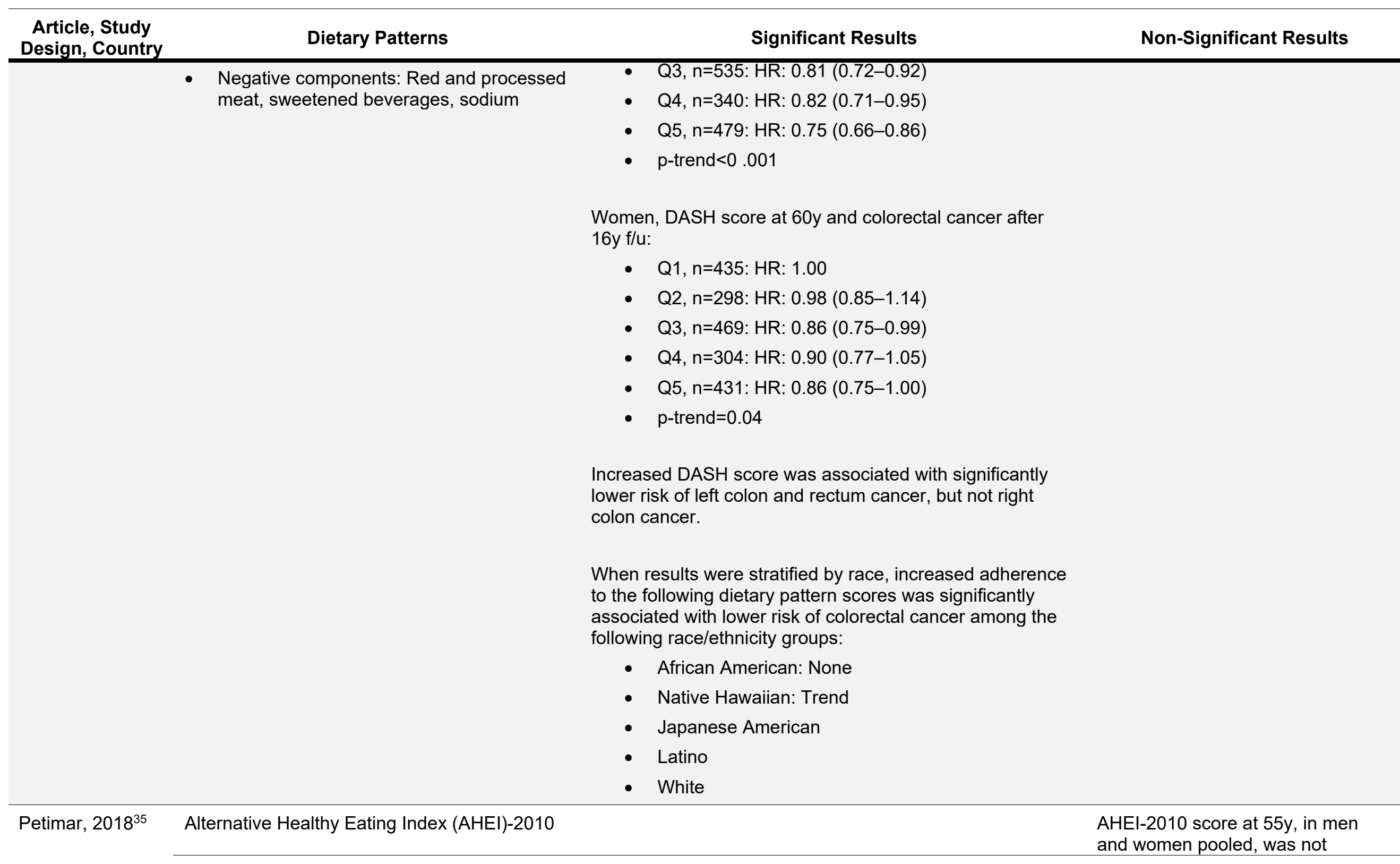




\begin{tabular}{|c|c|c|}
\hline $\begin{array}{c}\text { Article, Study } \\
\text { Design, Country }\end{array}$ & Dietary Patterns & Significant Results \\
\hline $\begin{array}{l}\text { PCS (Nurses' } \\
\text { Health Study, } \\
\text { Health } \\
\text { Professional } \\
\text { Follow-up Study) } \\
\text { United States }\end{array}$ & $\begin{array}{l}\text { - Positive components: Vegetables (not } \\
\text { potatoes, French fries), Fruit, Whole } \\
\text { Grains, Long-Chain Fats (EPA + DHA), } \\
\text { PUFA } \\
\text { - } \quad \text { Neutral components: Alcohol } \\
\text { - Negative components: Red and Processed } \\
\text { Meat, Sugar Sweetened Beverages and } \\
\text { Fruit Juice, Trans FA, Sodium }\end{array}$ & \\
\hline & $\begin{array}{l}\text { Alternative Mediterranean Diet Score (aMED) } \\
\text { - Positive components: Vegetables (not } \\
\text { potatoes), legumes, fruits, nuts, whole } \\
\text { grains, fish, MUFA/SFAs } \\
\text { - } \quad \text { Neutral components: Alcohol } \\
\text { - Negative components: Red and processed } \\
\text { meat }\end{array}$ & 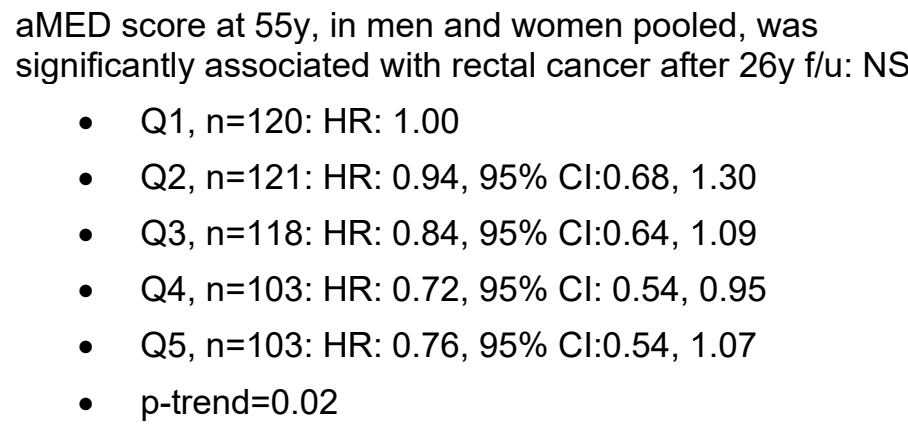 \\
\hline
\end{tabular}

When women and men were analyzed separately, results were similar in men, and no longer significant in women.

\section{DASH Score}

- Positive components: Vegetables (not potatoes), nuts and legumes, fruit and fruit juice, whole grains, low-fat dairy

- Negative components: Red and processed meat, sweetened beverages, sodium

\section{Non-Significant Results}

significantly associated with colorectal cancer (colorectal, colon, distal colon, proximal colon, rectal cancer) after $26 \mathrm{y} f / \mathrm{u}$

- In women only, results were similar

- In men only, increased AHEI2010 adherence was significantly associated with lower total colorectal colon cancer risk

aMED score at 55y, in men and women pooled, was not significantly associated with colorectal cancer (colorectal, colon, distal colon, proximal colon) after $26 \mathrm{y}$ f/u

- In women only, results were similar

- In men only, increased aMED adherence was significantly associated with lower total colorectal cancer risk

DASH score at 55y, in men and women pooled, was not significantly associated with colorectal cancer (colorectal, colon, distal colon, proximal colon, rectal cancer) after $26 y$ f/u

- In women only, results were similar

- In men only, increased DASH adherence was significantly 


\section{Roswall, $2015^{36}$ \\ PCS (Women's \\ Lifestyle and \\ Health cohort) \\ Sweden}

\section{Healthy Nordic food index (HNFI)}

- Positive components: Cabbage, root vegetables, apples and pears, rye bread, oatmeal, fish
HNFI score at 39y (categorical and continuous) was not significantly associated with colorectal cancer.
Schulpen, $2020^{44}$ alternate Mediterranean diet scores (aMED), and without alcohol (aMEDr)

Nested CaseControl Study

(Netherlands

Cohort Study

(NLCS))

The Netherlands
- Positive components: Vegetables (not potatoes), legumes, fruits, nuts, whole grains, fish, MUFA/SFAs

- Neutral components: Alcohol

- Negative components: Red and processed meat
In women, smoking status significantly modified the

relationship between aMEDr (per 2-pt increment) and colorectal cancer:

- Never smokers, $\mathrm{n}=256$ : HR: $1.00,95 \% \mathrm{Cl}: 0.88$ 1.13

- Former smokers, $n=350$ : HR: $0.78,95 \% \mathrm{Cl}: 0.63$, 0.98

- Current smokers, n=309: HR: $1.21,95 \% \mathrm{Cl}: 0.96$, 1.51

- $p$-interaction $=0.015$
Adherence to the aMED, with and without alcohol, at $61 \mathrm{y}$ was not significantly associated with risk of colorectal, colon, proximal, distal, or rectal cancer in men or women after $20.3 y$ f/u.

Results were also not significant when stratified by smoking status in men, alcohol consumption, body mass index, education, or family history of colorectal cancer.

Adherence to the WCRF/AICR, diet only score, with and without alcohol, at $61 \mathrm{y}$ was not significantly associated with risk of colorectal, colon, proximal, distal, or rectal cancer in men or women after $20.3 y$ f/u.

Results were also not significant when stratified by smoking status in men, alcohol consumption, body 


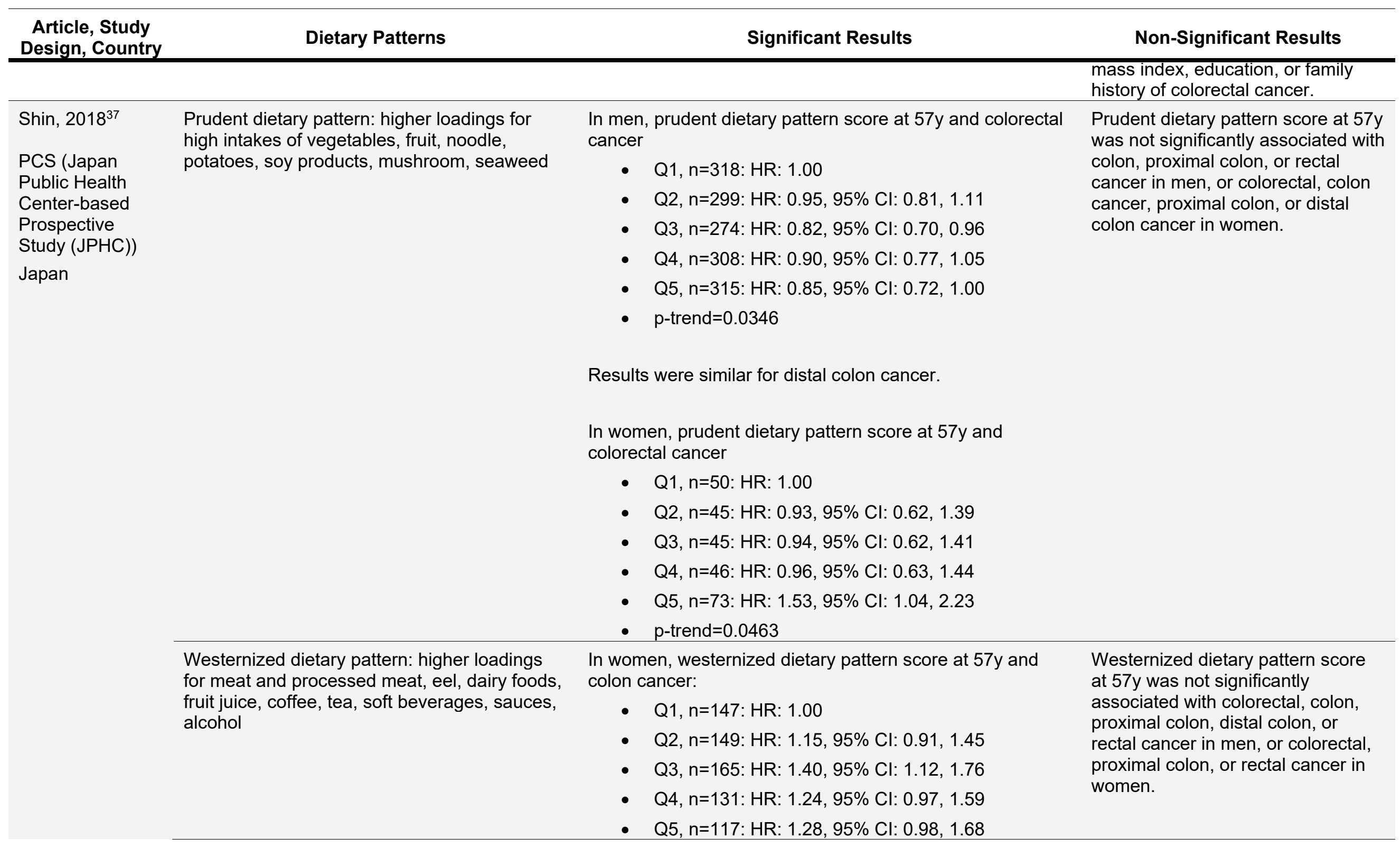




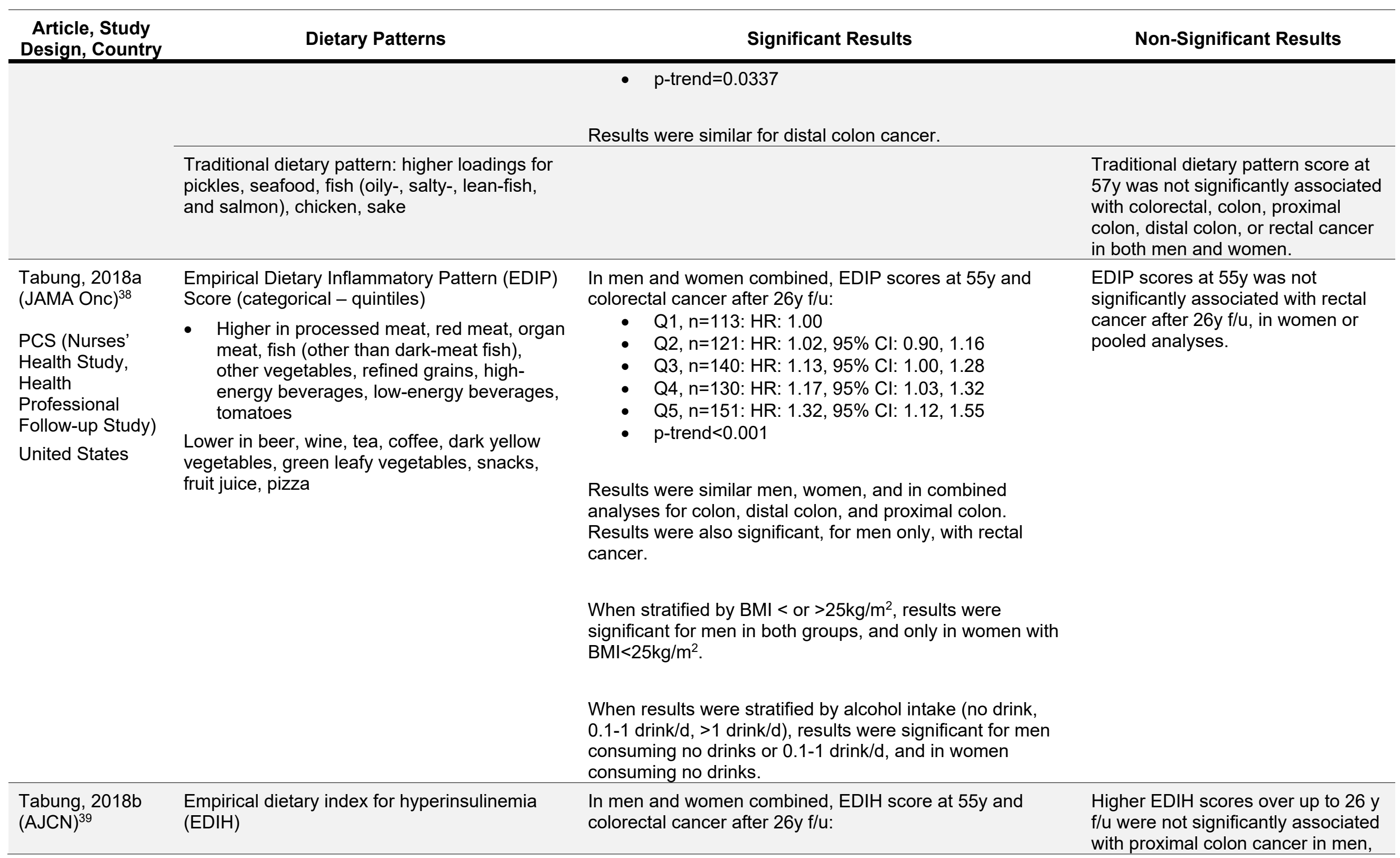




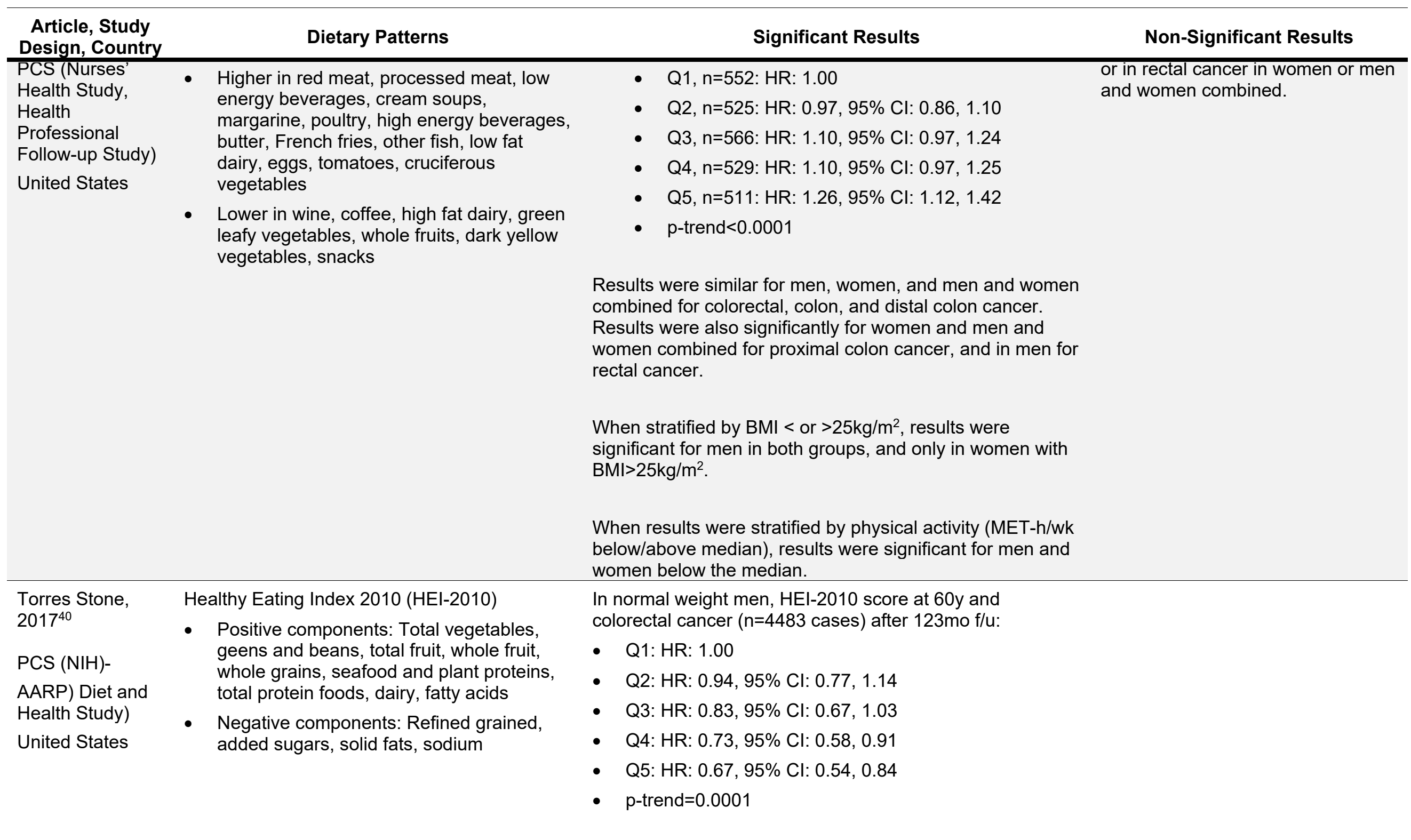


Results were also significant in overweight $(p<0.0001)$ and obese $(p=0.0394)$ men

When probablities were examined, in normal weight, overweight, and obese men, higher scores were associated with decreased risk of colorectal cancer.

In overweight women, $\mathrm{HEI}-2010$ score at 60y and colorectal cancer ( $n=2032$ cases) after $123 \mathrm{mo} \mathrm{f/u}$ :

- Q1: HR: 1.00

- Q2: HR: $0.87,95 \% \mathrm{Cl}: 0.65,1.16$

- Q3: HR: 0.94, 95\% Cl: 0.71, 1.25

- Q4: HR: 0.73, 95\% Cl: 0.55, 0.98

- Q5: HR: 0.64, 95\% Cl: 0.47, 0.86

- $p$-trend $=0.0001$

Results were not significant in normal weight $(p=0.1557)$ and obese $(p=0.0573)$ women

In overweight and obese women, higher score was associated with decreased risk of colorectal cancer.

Mediterranean diet score

- Positive components: Vegetables, legumes, fruit, cereals, fish MUFA+PUFA/SFA

- Neutral components: Alcohol

- Negative components: Meat, dairy products
In normal weight men, mediterranean diet score at $60 \mathrm{y}$ and colorectal cancer ( $n=4483$ cases $)$ after $123 \mathrm{mo} f / u$ :

- Q1: HR: 1.00

- Q2: HR: 0.79, 95\% Cl: 0.66, 0.96

- Q3: HR: $0.66,95 \%$ Cl: 0.54, 0.82

- Q4: HR: $0.67,95 \%$ Cl: 0.54, 0.84

- Q5: HR: 0.65, 95\% Cl: 0.51, 0.83

- $\quad p$-trend $=0.0004$
In normal weight, overweight, and obese women, mediterranean diet score at $60 \mathrm{y}$ was not significantly associated with colorectal cancer $(n=2032$ cases $)$ after $123 \mathrm{mo} / \mathrm{u}$. 
Results were also significant in overweight $(p=0.0013)$ and obese $(p=0.0508)$ men

When probablities were examined, in normal weight, overweight, and obese men, higher scores were associated with decreased risk of colorectal cancer.

Dietary Approaches to Stop Hypertension (DASH) score

- Positive components: Vegetables (not potatoes), nuts and legumes, fruit and fruit juice, whole grains, low-fat dairy

- Negative components: Red and processed meat, sweetened beverages, sodium
In normal weight men, DASH score at $60 y$ and colorectal cancer ( $n=4483$ cases) after $123 \mathrm{mo}$ f/u:

- Q1: HR: 1.00

- Q2: HR: 0.91, 95\% Cl: 0.08, 1.11

- Q3: HR: $0.79,95 \% \mathrm{Cl}: 0.64,0.99$

- Q4: HR: 0.83, 95\% Cl: 0.66, 1.04

- Q5: HR: 0.67, 95\% Cl: 0.54, 0.84

- $p$-trend $=0.0005$

Results were also significant in overweight men $(p<0.0001)$, but not in obese men $(p=0.0801)$

When probablities were examined, in normal weight, overweight, and obese men, higher scores were associated with decreased risk of colorectal cancer.

In normal weight women, DASH score at 60y and colorectal cancer ( $n=2032$ cases) after $123 \mathrm{mo} / \mathrm{f}$ :

- Q1: HR: 1.00

- Q2: HR: 0.86, 95\% Cl: 0.68, 1.09

- Q3: HR: 0.70, 95\% Cl: 0.53, 0.93

- Q4: HR: $0.86,95 \% \mathrm{Cl}: 0.66,1.13$ 


\begin{tabular}{|c|c|c|c|}
\hline $\begin{array}{c}\text { Article, Study } \\
\text { Design, Country }\end{array}$ & Dietary Patterns & Significant Results & Non-Significant Results \\
\hline & & $\begin{array}{l}\text { - Q5: HR: } 0.73,95 \% \text { Cl: } 0.56,0.95 \\
\text { - } \quad p \text {-trend }=0.0005 \\
\text { Results were also significant in obese women }(p=0.0128) \text {, } \\
\text { but not in overweight women ( } p=0.1256) \\
\text { When probablities were examined, in normal weight } \\
\text { women, higher DASH score was associated with } \\
\text { decreased risk of colorectal cancer. In overweight and } \\
\text { obese women, higher score was associated with } \\
\text { decreased risk of colorectal cancer. }\end{array}$ & \\
\hline \multirow[t]{2}{*}{$\begin{array}{l}\text { Vargas, } 2016^{41} \\
\text { PCS (Women's } \\
\text { Health Initiative } \\
\text { Observational } \\
\text { Study) } \\
\text { United States }\end{array}$} & $\begin{array}{l}\text { Healthy Eating Index (HEI)-2010 } \\
\text { Positive components: Total vegetables, } \\
\text { geens and beans, total fruit, whole fruit, } \\
\text { whole grains, seafood and plant proteins, } \\
\text { total protein foods, dairy, fatty acids } \\
\text { - Negative components: Refined grained, } \\
\text { added sugars, solid fats, sodium }\end{array}$ & $\begin{array}{l}\text { HEl-2010 score at } 63 y \text { and colorectal cancer after } 12.4 \text { y } \\
\text { f/u: } \\
\text { - Q1, n=209: HR: } 1.00 \\
\text { - } \quad \mathrm{Q} 2, \mathrm{n}=189: \mathrm{HR}: 0.88,95 \% \mathrm{Cl}: 0.72,1.08 \\
\text { - } \quad \mathrm{Q} 3, \mathrm{n}=175: \mathrm{HR}: 0.81,95 \% \mathrm{Cl}: 0.66,0.99 \\
\text { - } \quad \mathrm{Q} 4, \mathrm{n}=172: \mathrm{HR}: 0.77,95 \% \mathrm{Cl}: 0.63,0.95 \\
\text { - } \quad \mathrm{Q} 5, \mathrm{n}=166: \mathrm{HR}: 0.73,95 \% \mathrm{Cl}: 0.59,0.90 \\
\text { - } \quad \text {-trend=0.032 } \\
\text { Higher HEl-2010 score was significantly associated with } \\
\text { decreased risk of colon cancer, but not rectal cancer. }\end{array}$ & \\
\hline & $\begin{array}{l}\text { Alternative Healthy Eating Index (AHEI)-2010 } \\
\text { - Positive components: Vegetables (not } \\
\text { potatoes, French fries), Fruit, Whole } \\
\text { Grains, Long-Chain Fats (EPA + DHA), } \\
\text { PUFA } \\
\text { - Neutral components: Alcohol } \\
\text { - Negative components: Red and Processed } \\
\text { Meat, Sugar Sweetened Beverages and } \\
\text { Fruit Juice, Trans FA, Sodium }\end{array}$ & & $\begin{array}{l}\text { AHEI score at } 63 y \text { was not } \\
\text { significantly associated with risk of } \\
\text { colorectal, colon, and rectal cancer } \\
\text { after } 12.4 \text { y } f / u(p=0.427) \text {. }\end{array}$ \\
\hline
\end{tabular}




\begin{tabular}{|c|c|c|c|}
\hline $\begin{array}{c}\text { Article, Study } \\
\text { Design, Country }\end{array}$ & Dietary Patterns & Significant Results & Non-Significant Results \\
\hline & $\begin{array}{l}\text { Alternative Mediterranean Diet Score (aMED) } \\
\text { - Positive components: Vegetables (not } \\
\text { potatoes), legumes, fruits, nuts, whole } \\
\text { grains, fish, MUFA/SFAs } \\
\text { - Neutral components: Alcohol } \\
\text { - Negative components: Red and processed } \\
\quad \text { meat }\end{array}$ & & $\begin{array}{l}\text { aMED score at } 63 \mathrm{y} \text { was not } \\
\text { significantly associated with risk of } \\
\text { colorectal, colon, and rectal cancer } \\
\text { after } 12.4 \text { y } f / u(p=0.217) \text {. }\end{array}$ \\
\hline & $\begin{array}{l}\text { DASH Score } \\
\text { - Positive components: Vegetables (not } \\
\text { potatoes), nuts and legumes, fruit and fruit } \\
\text { juice, whole grains, low-fat dairy } \\
\text { - Negative components: Red and processed } \\
\text { meat, sweetened beverages, sodium }\end{array}$ & 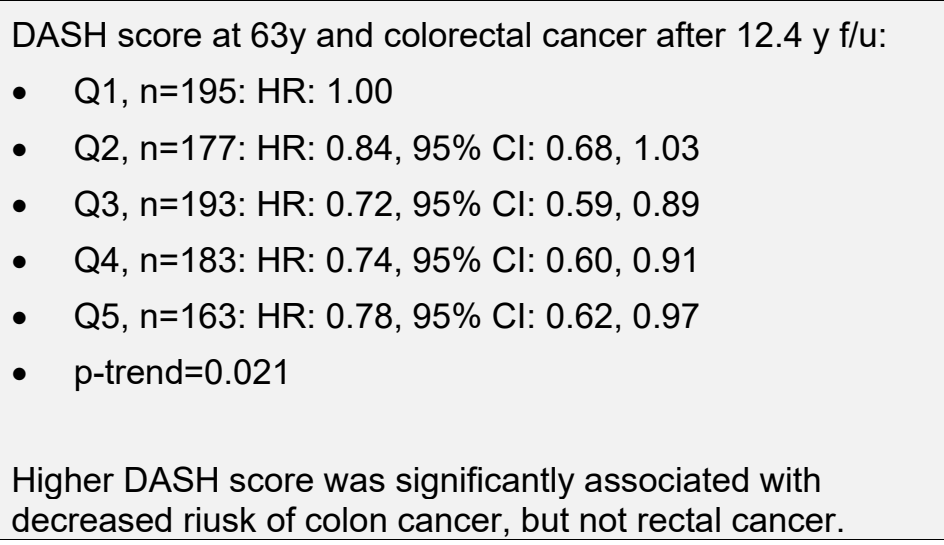 & \\
\hline $\begin{array}{l}\text { Voortman, } 2017^{24} \\
\text { PCS (Rotterdam } \\
\text { Study) } \\
\text { The Netherlands }\end{array}$ & $\begin{array}{l}\text { Dutch Dietary Guidelines } 2015 \text { score } \\
\text { - Positive components: Vegetables, } \\
\text { legumes, fruit, nuts, whole grains, fish, } \\
\text { dairy products, unsaturated fats and oils, } \\
\text { tea } \\
\text { - Negative components: Replace refined } \\
\text { grains with whole-grain products, red meat, } \\
\text { processed meat, alcohol, sodium }\end{array}$ & $\begin{array}{l}\text { Dutch Dietary Guidelines } 2015 \text { score and colorectal cancer } \\
\text { (n=324) after } 11 \mathrm{y} f / \mathrm{u}: \mathrm{HR}: 0.90,95 \% \mathrm{Cl}: 0.84,0.96), \mathrm{p}<0.05\end{array}$ & \\
\hline $\begin{array}{l}\text { Vulcan, } 2019^{42} \\
\text { PCS (Malmö Diet } \\
\text { and Cancer } \\
\text { Study) }\end{array}$ & $\begin{array}{l}\text { Colorectal Diet Quality Index (CDQI) } \\
\text { - } \quad \text { Positive components: Fiber, dairy products } \\
\text { - } \quad \text { Negative components: Processed meat }\end{array}$ & $\begin{array}{l}\text { CDQI score at } 59 y \text { and colorectal cancer }(n=923) \text { after } \\
502136 \text { person years f/u: } \\
\text { - } 0-3, n=135: \text { HR: } 1.00 \\
\text { - } \quad 4-6, n=222: \text { HR: } 0.77,95 \% \text { Cl: } 0.65,0.91\end{array}$ & \\
\hline
\end{tabular}


- 7-9, n=187: HR: $0.74,95 \%$ Cl: $0.61,0.89$

- 10-12, n=46: HR: 0.57, 95\% Cl: $0.43,0.75$

- $\quad$-trend $<0.001$

Results were similar for colon and rectal cancer, and when women and men were analyzed seperately. 
Table 7. Risk of bias for randomized controlled trials examining dietary patterns and colorectal cancer|xii, Ixiii

\begin{tabular}{|l|c|c|c|c|c|c|}
\hline & Randomization & $\begin{array}{c}\text { Deviations from } \\
\text { intended } \\
\text { interventions - } \\
\text { effect of } \\
\text { assignment }\end{array}$ & $\begin{array}{c}\text { Deviations from } \\
\text { intended } \\
\text { interventions - } \\
\text { per-protocol }\end{array}$ & $\begin{array}{c}\text { Missing } \\
\text { outcome data }\end{array}$ & $\begin{array}{c}\text { Outcome } \\
\text { measurement }\end{array}$ & $\begin{array}{c}\text { Selection of the } \\
\text { reported result }\end{array}$ \\
\hline Prentice, 20191 & Low & Low & Some & Low & Low & Low \\
\hline Thomson, 20142 & Low & Low & Some & Low & Low & Low \\
\hline
\end{tabular}

Ixii A detailed description of the methodology used for assessing risk of bias is available on the NESR website: https://nesr.usda.gov/2020-dietary-guidelinesadvisory-committee-systematic-reviews and in Part C of the following reference: Dietary Guidelines Advisory Committee. 2020. Scientific Report of the 2020 Dietary Guidelines Advisory Committee: Advisory Report to the Secretary of Agriculture and the Secretary of Health and Human Services. U.S. Department of Agriculture, Agricultural Research Service, Washington, DC.

xiiipossible ratings of low, some concerns, or high determined using the "Cochrane Risk-of-bias 2.0" (RoB 2.0) (August 2016 version)" (Higgins JPT, Sterne JAC, Savović J, Page MJ, Hróbjartsson A, Boutron I, Reeves B, Eldridge S. A revised tool for assessing risk of bias in randomized trials In: Chandler J, McKenzie J, Boutron I, Welch V (editors). Cochrane Methods. Cochrane Database of Systematic Reviews 2016, Issue 10 (Suppl 1).

dx.doi.org/10.1002/14651858.CD201601.) 
Table 8. Risk of bias for observational studies examining dietary patterns and colorectal cancer lxiv

\begin{tabular}{|c|c|c|c|c|c|c|c|}
\hline Article & Confounding & $\begin{array}{l}\text { Selection of } \\
\text { participants }\end{array}$ & $\begin{array}{l}\text { Classification } \\
\text { of exposures }\end{array}$ & $\begin{array}{c}\text { Deviations from } \\
\text { intended } \\
\text { exposures }\end{array}$ & Missing data & $\begin{array}{c}\text { Outcome } \\
\text { measurement }\end{array}$ & $\begin{array}{l}\text { Selection of the } \\
\text { reported result }\end{array}$ \\
\hline Boden, 201927 & Serious & Moderate & Low & Serious & Serious & Low & Moderate \\
\hline Cheng, $2018^{43}$ & Serious & Moderate & Low & Serious & Moderate & Low & Moderate \\
\hline Deschasaux, $2018^{4}$ & Serious & Moderate & Low & Serious & Moderate & Low & Moderate \\
\hline Fasanelli, $2017^{28}$ & Critical & Moderate & Low & Serious & Moderate & Moderate & Serious \\
\hline Fiolet, $2018^{7}$ & Serious & Moderate & Moderate & Serious & Moderate & Low & Moderate \\
\hline Jones, $2017^{29}$ & Serious & Moderate & Low & Serious & Serious & Low & Moderate \\
\hline Kumagai, 2014 ${ }^{30}$ & Serious & Moderate & Moderate & Serious & Serious & Low & Moderate \\
\hline Lavalette, $2018^{16}$ & Serious & Moderate & Low & Serious & Serious & Low & Moderate \\
\hline Liu, $2017^{31}$ & Serious & Moderate & Low & Moderate & Moderate & Low & Moderate \\
\hline Mehta, 201732 & Serious & Moderate & Moderate & Moderate & Moderate & Low & Moderate \\
\hline Orlich, $2015^{33}$ & Serious & Moderate & Low & Serious & Moderate & Low & Moderate \\
\hline Park, $2017^{34}$ & Serious & Moderate & Low & Serious & Moderate & Low & Moderate \\
\hline Petimar, 2018 ${ }^{35}$ & Serious & Moderate & Low & Moderate & Moderate & Low & Moderate \\
\hline Roswall, $2015^{36}$ & Critical & Moderate & Low & Serious & Moderate & Low & Moderate \\
\hline Schulpen, $2020^{44}$ & Serious & Serious & Low & Serious & Moderate & Low & Serious \\
\hline Shin, $2018^{37}$ & Critical & Moderate & Moderate & Serious & Moderate & Low & Moderate \\
\hline Tabung, 2018a ${ }^{38}$ & Serious & Moderate & Low & Moderate & Moderate & Low & Moderate \\
\hline Tabung, 2018b 39 & Serious & Moderate & Low & Moderate & Moderate & Low & Moderate \\
\hline Torres Stone, $2017^{40}$ & Serious & Serious & Low & Serious & Serious & Low & Moderate \\
\hline Vargas, $2016^{41}$ & Serious & Moderate & Low & Serious & Moderate & Low & Moderate \\
\hline Voortman, $2017^{24}$ & Serious & Moderate & Low & Serious & Moderate & Low & Moderate \\
\hline Vulcan, $2019^{42}$ & Serious & Low & Serious & Serious & Low & Low & Moderate \\
\hline
\end{tabular}

lxiv Possible ratings of low, moderate, serious, critical, or no information determined using the "Risk of Bias for Nutrition Observational Studies" tool (RoBNObs) (Dietary Guidelines Advisory Committee. 2020. Scientific Report of the 2020 Dietary Guidelines Advisory Committee: Advisory Report to the Secretary of Agriculture and the Secretary of Health and Human Services. U.S. Department of Agriculture, Agricultural Research Service, Washington, DC.) 
Table 9. Description of studies that examined the relationship between dietary patterns and lung cancerlxv

\section{Study and \\ Participant \\ Characteristics \\ Anic, $2016^{45}$ \\ PCS (NIH-AARP Diet and Health Study) \\ United States}

\section{Intervention/Exposure and Outcomes Results}

\section{Confounding, Study Limitations, and} Summary of Findings
Analytic N: 460770

Participants were $40 \%$ female, $\sim 62 \mathrm{y}(50-71 \mathrm{y})$, $\sim 92 \%$ white,

$\sim 27 \mathrm{~kg} / \mathrm{m} 2, \sim 40 \%$ never smokers, $\sim 15 \mathrm{~g} / \mathrm{d}$ for men and $\sim 5$ $\mathrm{g} / \mathrm{d}$ for women alcohol, $\sim 37 \%$ college graduates, $\sim 19 \%$ $\geq 5 \mathrm{x} /$ wk physical activity

\section{Dietary patterns:}

- Healthy Eating Index (HEI)-2010 Ixvi, categorical (quintiles)

- Positive components: Total vegetables, geens and beans, total fruit, whole fruit, whole grains, seafood and plant proteins, total protein foods, dairy, fatty acids

- Negative components: Refined grained, added sugars, solid fats, sodium

- Alternative Healthy Eating Index (AHEI)-2010Ixvii, categorical (quintiles)

- Positive components: Vegetables (not potatoes, French fries), Fruit, Whole Grains, Long-Chain Fats (EPA + DHA), PUFA

- Neutral components: Alcohol

\section{Significant:}

$\mathrm{HEI}-2010$ score at $62 \mathrm{y}$ and lung cancer after $10.5 \mathrm{y}$ f/u:

- $\mathrm{Q} 1, \mathrm{n}=3076: 1.00$

- Q2, $n=1947:$ HR $0.90,95 \% \mathrm{Cl}: 0.84,0.95$

- Q3, $n=1640:$ HR $0.91,95 \% \mathrm{Cl}: 0.86,0.97$

- Q4, n=1436: HR 0.92, 95\% Cl: 0.86, 0.98

- $\quad$ $5, n=1173: \mathrm{HR} 0.83,95 \% \mathrm{Cl}: 0.77,0.89$

- $p$-trend $<0.0001$

When analyzed by smoking status, results remained significant for former and current smokers, but were no longer significant for never smokers.

AHEI-2010 score at 62y and lung cancer after 10.5y f/u:

- Q1, n=2448: HR: 1.00

- Q2, n=2004: HR 0.96, 95\% Cl: 0.90, 1.02

\section{Key confounders accounted for:}

Sex, age, race/ethnicity, education, alcohol intake, physical activity, smoking, BMI

Other:

Total energy intake

\section{Limitations:}

- Did not account for family history of the cancer outcome, lung disease, environmental exposures to lung carcinogens

- Exposure occurred prior to start of $\mathrm{f} / \mathrm{u}$

- Only assessed dietary intake once at baseline; did not account for possible changes in dietary intake over $\mathrm{f} / \mathrm{u}$

- Did not account for missing data

- No preregistered statistical plan;

Ixv Abbreviations : AHEI-2010, Alternative Healthy Eating Index-2010; aMED, Alternative Mediterranean Diet Score ; BMI, body mass index; d, day; DASH, Dietary Approaches to Stop Hypertension; DP, Dietary pattern; \%E, \% of energy; EPIC, European Prospective Investigation into Cancer and Nutrition; FFQ, food frequency questionnaire; FSAm-NPS, Nutrient Profiling System of the British Food Standards Agency (modified version); f/u, follow-up; HR, hazard ratio; MDS, Mediterranean Diet Score; mMEDr, modified Mediterranean diet scores; mMEDr, modified Mediterranean diet scores without alcohol; mo, month or months; N/A, Not applicable; NIH, National Institutes of Health; NS, Not significant; NR, Not reported; PCS, prospective cohort study; pt, point; RCT, randomized controlled trial; WCRF/AICR, World Cancer Research Fund/American Institute for Cancer Research; wk, weeks; y, year

IxviGuenther PM, Casavale KO, Reedy J, Kirkpatrick SI, Hiza HA, Kuczynski KJ et al. Update of the Healthy Eating Index: HEI-2010. J Acad Nutr Diet 2013; 113: 569-580.

Ixvii Chiuve SE, Fung TT, Rimm EB, Hu FB, McCullough ML, Wang M et al. Alternative dietary indices both strongly predict risk of chronic disease. J Nutr 2012; 142: 1009-1018. 


\begin{tabular}{|c|c|c|c|}
\hline $\begin{array}{l}\text { Study and } \\
\text { Participant } \\
\text { Characteristics }\end{array}$ & Intervention/Exposure and Outcomes & Results & $\begin{array}{l}\text { Confounding, Study Limitations, and } \\
\text { Summary of Findings }\end{array}$ \\
\hline $\begin{array}{l}\text { Excluded participants } \\
\text { whose questionnaire } \\
\text { was completed by a } \\
\text { proxy, with prevalent } \\
\text { cancer, cancer cause } \\
\text { of death record } \\
\text { but no cancer registry } \\
\text { data, with end-stage } \\
\text { renal disease, } \\
\text { implausible energy } \\
\text { intake, missing } \\
\text { information on tobacco } \\
\text { smoking ( 19\% of } \\
\text { original sample) }\end{array}$ & 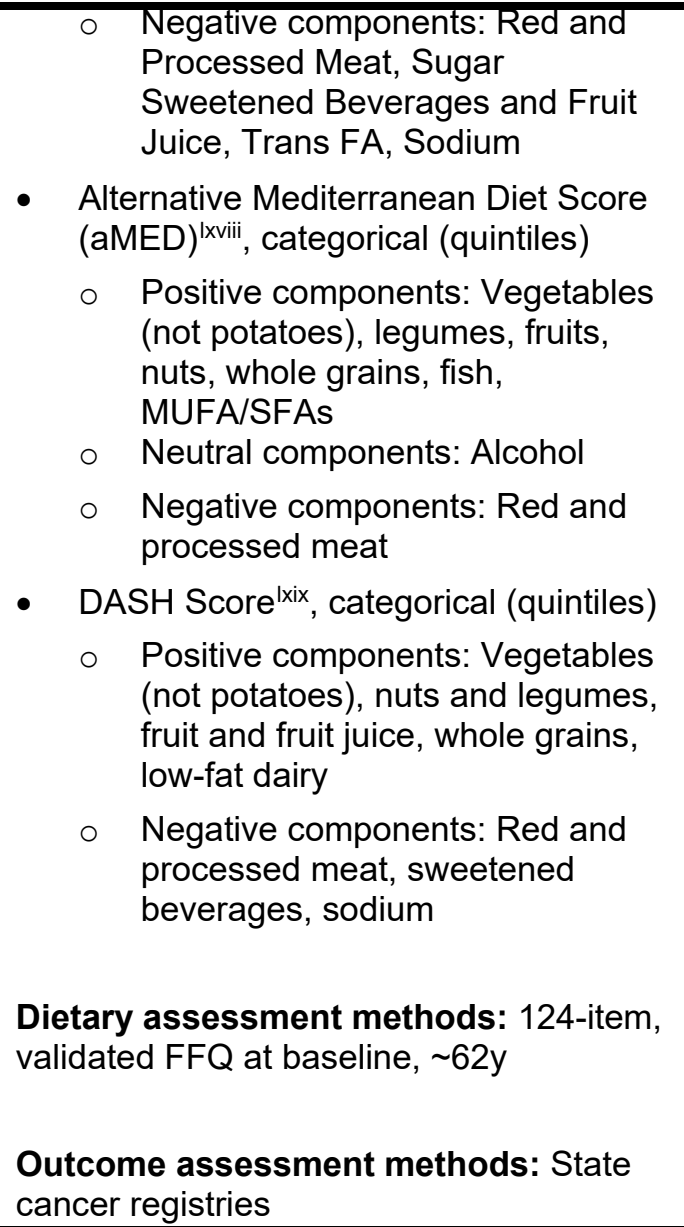 & 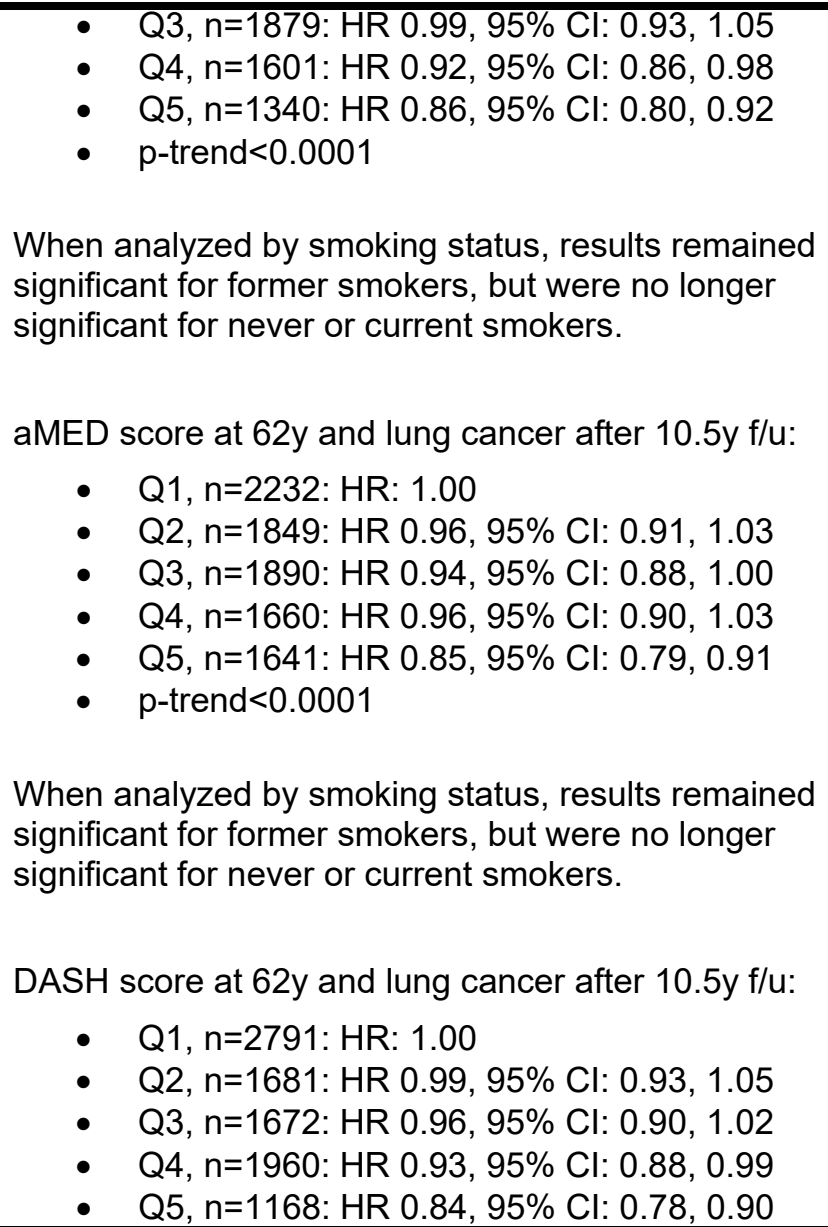 & $\begin{array}{l}\quad \text { potential for selective outcome } \\
\text { reporting } \\
\text { Funding Sources: NIH } \\
\text { Summary: Higher adherence to the HEI- } \\
\text { 2010, AHEI-2010, aMED, and DASH } \\
\text { scores at 62y were all associated with } \\
\text { significantly lower risk of lung cancer after } \\
\text { 10.5y f/u. } \\
\text { When analyzed by smoking status, AHEI- } \\
\text { 2010, aMED, and DASH score results } \\
\text { remained significant for former smokers, } \\
\text { but were no longer significant for never or } \\
\text { current smokers. For HEI-2010, results } \\
\text { remained significant for former and } \\
\text { current smokers, but were no longer } \\
\text { significant for never smokers. }\end{array}$ \\
\hline
\end{tabular}

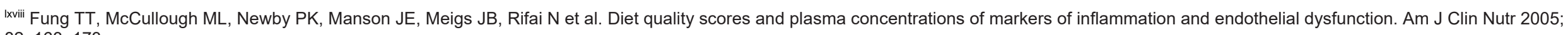
82: 163-173.

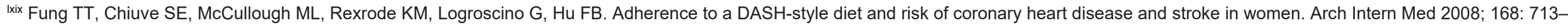

720. 


\section{Study and \\ Participant \\ Characteristics}

Results
Confounding, Study Limitations, and

Summary of Findings
- $\quad \mathrm{p}$-trend<0.0001

When analyzed by smoking status, results remained significant for former smokers, but were no longer significant for never or current smokers.

\section{Non-Significant: N/A}

\section{Boden, 201927}

PCS (Vasterbotten

Intervention

Programme)

Sweden

Analytic N: 35393

Participants were $52 \%$ female, $\sim 46 \mathrm{yo}, \sim 15 \%$ obese, $\sim 17 \%$ current smoker, $\sim 70 \%$ no post-secondary education, $\sim 39 \%$ low physical activity

Excluded participants with prevalent cancer, insufficient dietary data, implausible food or energy intakes,

\section{Dietary patterns:}

- Mediterranean diet score (MDS) ${ }^{1 \mathrm{xx}}$ per tertile increase

- Positive components: Vegetables and potatoes, fruit and fresh juices, wholegrain cereals, fish and fish products, MUFA+PUFA/SFA,

- Moderation components: Alcohol

- Negative components: Meat and meat products, dairy products

Dietary assessment methods: 84 -item and 64-66-item, validated, FFQs, at least 2 measures, $<2 y$ apart at baseline, age $\sim 46 y$

\section{Outcome assessment methods:}

Swedish Cancer Registry

\section{Key confounders accounted for:}

Sex, age, education, alcohol intake, physical activity, smoking, BMI

\section{Non-Significant:}

All participants, MDS score, per tertile increase, at $46 y$ and lung cancer $(n=442)$ after 15y f/u: HR: 0.90, $95 \% \mathrm{Cl}: 0.80,1.01$

Men, MDS score, per tertile increase, at 46y and lung cancer $(n=210)$ after $15 \mathrm{y} f / \mathrm{u}$ : HR: $0.86,95 \% \mathrm{Cl}$ : $0.72,1.03$

Women, MDS score, per tertile increase, at 46y and lung cancer $(n=232)$ after $15 y \mathrm{f} / \mathrm{u}$ : HR: $0.94,95 \% \mathrm{Cl}$ : $0.79,1.10$

\section{Other:}

\section{Energy intake}

\section{Limitations:} lung disease, environmental exposures to lung carcinogens
- Did not account for race/ethnicity, family history of the cancer outcome,

- Exposure occurred prior to start of $\mathrm{f} / \mathrm{u}$

- Only assessed dietary intake in first $2 y$ from baseline; did not account for possible changes in dietary intake over f/u

- Did not account for missing data

- No preregistered statistical plan; potential for selective outcome reporting

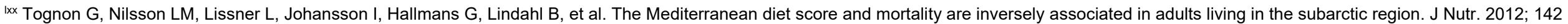
(8):1547-53. 


\section{Study and \\ Participant}

Characteristics

Intervention/Exposure and Outcomes

Results

Confounding, Study Limitations, and Summary of Findings

implausible

anthropometric data,

cancer diagnosed

within $1 \mathrm{y}$ year of last

measurement, single

dietary measure

( $65 \%$ of original

sample)

\section{Deschasaux, 2018 \\ PCS (European \\ Prospective \\ Investigation into \\ Cancer and Nutrition \\ (EPIC)) \\ Denmark, France, \\ Germany, Greece, \\ Italy, the \\ Netherlands, \\ Norway, Spain, \\ Sweden, UK}

Analytic N: 471495

Participants were $70 \%$ female, 51 yo, $\sim 25.4$

$\mathrm{kg} / \mathrm{m}^{2} \mathrm{BMI}, 43 \%$

never-smokers, $\sim 5.3$

\section{Dietary patterns:}

Nutrient Profiling System of the British Food Standards Agency (modified version) (FSAm-NPS) score, categorical (quintiles) and continuous (per $2 \mathrm{pt}$ increment) [ -15 (most healthy) to +40 (least healthy)]

- Overall diet score assigned based on energy, sugar, saturated fatty acid, sodium, fibres, proteins, and fruits/vegetables/legumes/nuts.

- Higher FSAm-NPS score had higher intakes of alcohol, energy and red and processed meat, lower intakes of dietary fibres, vegetables, fruit, fish, and lean meat

Dietary assessment methods: FFQs or 7-day diet records, validated, at baseline, age $\sim 51 \mathrm{y}$

\section{Significant:}

FSAm-NPS score at 51y in men and risk of lung cancer after $15.3 \mathrm{y}$ f/u:

- Q1, n=297: HR: 1.00

- Q2, n=336: HR: 1.21, 95\% Cl: 1.02, 1.43

- Q3, n=343: HR: 1.21, 95\% Cl: 1.02, 1.44

- $Q 4, n=415: H R: 1.31,95 \%$ Cl: 1.10, 1.60

- $Q 5, n=485: H R: 1.26,95 \%$ Cl: 1.06, 1.51

- $p$-trend $=0.02$

\section{Non-Significant:}

FSAm-NPS score at 51y in men and women combined and risk of lung cancer after 15.3y f/u:

- Q1, n=640: HR: 1.00

- Q2, n=684: HR: 1.05, 95\% Cl: 0.94, 1.17

- $\quad \mathrm{Q} 3, \mathrm{n}=702: \mathrm{HR}: 1.03,95 \% \mathrm{Cl}: 0.92,1.16$
Funding Sources: The Cancer Research

Fund in Northern Sweden, Arctic

Research Center (Arcum) at Umeå

University, Ostersunds Hospital, Swedish

Cancer Society, Region Vasterbotten,

Swedish Research Council for Health,

Working Life and Welfare, Swedish

Research Council

Summary: Mediterranean diet score at $46 y$ was not associated with risk of lung cancer after $15 \mathrm{y} / \mathrm{f}$.

\section{Key confounders accounted for:}

Sex, age, education, alcohol intake, physical activity, smoking, BMI, height

Other:

Center, energy intake

\section{Limitations:}

- Did not account for race/ethnicity, lung disease, environmental exposures to lung carcinogens, family history of the cancer

- Exposure occurred prior to start of $\mathrm{f} / \mathrm{u}$

- Only assessed dietary intake once at baseline; did not account for possible changes in dietary intake over f/u

- Did not account for missing data

- No preregistered statistical plan; potential for selective outcome reporting 


\begin{tabular}{|c|c|c|c|}
\hline $\begin{array}{l}\text { Study and } \\
\text { Participant } \\
\text { Characteristics }\end{array}$ & Intervention/Exposure and Outcomes & Results & $\begin{array}{l}\text { Confounding, Study Limitations, and } \\
\text { Summary of Findings }\end{array}$ \\
\hline $\begin{array}{l}\text { g/d alcohol, } \sim 20.9 \% \\
\text { inactive; } 17.9 \% \text { active. } \\
\text { Excluded those with } \\
\text { prevalent cancer; } \\
\text { cancer diagnosis in } \\
\text { first } 2 y \text { of f/u }(\sim 10 \% \text { of } \\
\text { original sample) }\end{array}$ & $\begin{array}{l}\text { Outcome assessment methods: Record } \\
\text { linkage with population-based cancer } \\
\text { registries, health insurance records, } \\
\text { pathology registries, and f/u with study } \\
\text { participants }\end{array}$ & $\begin{array}{l}\text { - Q4, n=782: HR: } 1.09,95 \% \mathrm{Cl}: 0.97,1.22 \\
\text { - Q5, n=846: HR: } 1.06,95 \% \mathrm{Cl}: 0.94,1.20 \\
\text { p-trend=0.3 } \\
\text { Continuous, per 2pt increment, n=3654: HR: } 1.01 \text {, } \\
\text { 95\% Cl: } 0.97,1.04 ; \text {-trend=0.7 } \\
\text { When men and women were analyzed separately, } \\
\text { results were also non-significant in women. }\end{array}$ & $\begin{array}{l}\text { Funding Sources: French National } \\
\text { Cancer Institute, European Commission, } \\
\text { the International Agency for Research on } \\
\text { Cancer } \\
\text { Summary: Nutrient Profiling System of the } \\
\text { British Food Standards Agency (modified } \\
\text { version) (FSAm-NPS) score at } 51 y \text { was } \\
\text { not significantly associated with risk of } \\
\text { lung cancer after 15.3y flu when men and } \\
\text { women were combined, and for women } \\
\text { only. In men, higher FSAm-NPS score } \\
\text { was significantly associated with } \\
\text { increased risk of lung cancer. }\end{array}$ \\
\hline $\begin{array}{l}\text { Hodge, } 2016^{46} \\
\text { PCS (Melbourne } \\
\text { Collaborative Cohort } \\
\text { Study) } \\
\text { Australia } \\
\text { Analytic N: } 35303 \\
\text { Participants were } \\
\sim 60 \% \text { female, } 40-69 y, \\
\sim 62 \% \text { overweight or } \\
\text { obese, 59\% never } \\
\text { smokers, 55\% low } \\
\text { alcohol intake, } 22 \% \\
\text { no physcial activity, } \\
\sim 23 \% \text { had a degree } \\
\text { after high school }\end{array}$ & $\begin{array}{l}\text { Dietary patterns: } \\
\begin{array}{l}\text { - Mediterranean diet score, categorical } \\
(0-3,4-6,7-9) \text { and continuous } \\
\circ \quad \text { Positive components: Vegetables, } \\
\text { legumes, fruit and nuts, cereals, } \\
\text { fish, olive oil } \\
\circ \quad \text { Moderation components: Alcohol } \\
\circ \quad \text { Negative components: Red and } \\
\text { processed meat, dairy products }\end{array}\end{array}$ & $\begin{array}{l}\text { MDS, continuous, and lung cancer after } 18 \mathrm{y} \text { f/u: HR: } \\
0.89,95 \% \mathrm{Cl}: 0.81,0.96 \\
\text { When analyzed by smoking status, results remained } \\
\text { significant in current smokers, but were no longer } \\
\text { significant in never and former smokers. } \\
\text { Non-Significant: N/A }\end{array}$ & $\begin{array}{l}\text { Key confounders accounted for: } \\
\text { Sex, age, race/ethnicity, education, SES } \\
\text { index, alcohol intake, physical activity, } \\
\text { Smoking, BMI } \\
\text { Other: } \\
\text { Energy intake, country } \\
\text { Limitations: } \\
\text { - Did not account for family history of } \\
\text { the cancer outcome, lung disease, } \\
\text { environmental exposures to lung } \\
\text { carcinogens } \\
\text { - Potential for selection bias due } \\
\text { exclusion of participants with several } \\
\text { chronic diseases at baseline } \\
\text { - Exposure occurred prior to start of f/u } \\
\text { - Only assessed dietary intake once at }\end{array}$ \\
\hline
\end{tabular}




\section{Study and \\ Participant \\ Characteristics \\ Excluded those with \\ prevalent cancer, \\ diabetes, heart attack, \\ or angina; no dietary \\ data; missing values \\ for confounders: \\ implausible energy \\ intake $(-15 \%$ of \\ original sample)}

Intervention/Exposure and Outcomes

Results

Confounding, Study Limitations, and Summary of Findings

baseline; did not account for possible changes in dietary intake over f/u

- Did not account for missing data

- $\quad$ No preregistered statistical plan; potential for selective outcome reporting

Funding Sources: VicHealth and Cancer Council Victoria, Australian National Health and Medical Research Council, Cancer Council Victoria

Summary: Higher MDS score at 40-69y was associated with significantly lower risk of lung cancer after $18 \mathrm{y}$ f/u. When analyzed by smoking status, results remained significant in current smokers, but were no longer significant in never and former smokers.

Kane-Diallo, 2018
PCS (NutriNet-Sante
study)
France

\section{Dietary patterns:}

"Pro plant-based" dietary score,

categorical (tertiles) range of 12-60 points; higher score = higher contribution of plant foods

- Higher in vegetables, legumes, fruits, cereal products, potatoes, nuts, vegetables oils

- Lower in red and processed meat, eggs, animal fat, dairy products, seafood

\section{Significant:}

"Pro plant-based" dietary score at 57y and risk of lung cancer after $4.3 \mathrm{y} / \mathrm{u}$ :

- T1, n=28: HR: 1.00

- T2, n=25: HR: $0.82,95 \%$ Cl: $0.48,1.43$

- T3, n=15: HR: 0.47, 95\% Cl: 0.24, 0.90

- $p$-trend $=0.02$

\section{Non-Significant: N/A}

\section{Key confounders accounted for:}

Sex, age, education, alcohol intake (in adults), physical activity, smoking, height, $\mathrm{BMI}$, family history of the cancer outcome

\section{Other:}

Energy intake without alcohol, number of 24-hr dietary records, lipids intake

\section{Limitations:}

- Did not account for race/ethnicity, lung disease, environmental exposures to lung carcinogens 


\begin{tabular}{|c|c|c|c|}
\hline $\begin{array}{l}\text { Study and } \\
\text { Participant } \\
\text { Characteristics }\end{array}$ & Intervention/Exposure and Outcomes & Results & $\begin{array}{l}\text { Confounding, Study Limitations, and } \\
\text { Summary of Findings }\end{array}$ \\
\hline $\begin{array}{l}\text { high or moderate } \\
\text { physical activity, } \sim 51 \% \\
\text { family history of } \\
\text { cancer }\end{array}$ & $\begin{array}{l}\text { Dietary assessment methods: } 3,24- \\
\text { hour dietary records, assessed during the } \\
\text { first year of f/u, at age } \sim 57 \mathrm{y}\end{array}$ & & $\begin{array}{l}\text { - } \\
\text { - Onposure occurred prior to start of } \mathrm{f} / \mathrm{u} \\
\text { first year of f/u; did not account for } \\
\text { possible changes in dietary intake } \\
\text { over f/u }\end{array}$ \\
\hline \multirow{3}{*}{$\begin{array}{l}\text { Excluded those with } \\
\text { prevalent cancer; }<3 \\
24 \text {-hr recalls within the } \\
\text { first year of } \mathrm{f} / \mathrm{u} \text {; } \\
\text { missing } \mathrm{f} / \mathrm{u} \text { data; } \\
\text { implausible energy } \\
\text { intake; }<45 \mathrm{y}(21 \% \text { of } \\
\text { original sample) }\end{array}$} & $\begin{array}{l}\text { Outcome assessment methods: } \\
\text { Participant report, medical record review, } \\
\text { pathological reports, linked to health } \\
\text { insurance and mortality databases }\end{array}$ & & $\begin{array}{l}\text { - Did not account for missing data } \\
\text { - No preregistered statistical plan; } \\
\text { potential for selective outcome } \\
\text { reporting }\end{array}$ \\
\hline & & & $\begin{array}{l}\text { Funding Sources: Ministere de la Sante, } \\
\text { Institut de Veille Sanitaire, Institut } \\
\text { National de la Prevention et de } \\
\text { l'Education pour la Sante, Region lle-de- } \\
\text { France, Institut National de la Sant_e et } \\
\text { de la Recherche Medicale, Institut } \\
\text { National de la Recherche Agronomique, } \\
\text { Conservatoire National des Arts et } \\
\text { Metiers, The French National Cancer } \\
\text { Institute }\end{array}$ \\
\hline & & & $\begin{array}{l}\text { Summary: Higher "pro plant-based" } \\
\text { dietary score at } 57 y \text { was associated with } \\
\text { significantly decreased risk of lung cancer } \\
\text { after } 4.3 y \text { f/u. }\end{array}$ \\
\hline Maisonneuve, $2016^{47}$ & Dietary patterns: & Significant: & Key confounders accounted for: \\
\hline $\begin{array}{l}\text { PCS (COSMOS } \\
\text { study) }\end{array}$ & $\begin{array}{l}\text { Alternative Mediterranean Diet Score } \\
(\text { aMED) })^{1 \times x i} \text {, categorical }(0-1,2-4,5-7 \text {, } \\
8-9)\end{array}$ & $\begin{array}{l}\text { aMED score at >50y and lung cancer after } 8.5 \mathrm{y} \text { f/u:: } \\
\text { - } \quad 0-1, \mathrm{n}=16: \mathrm{HR}: 1.00 \\
\text { - } 2-4, \mathrm{n}=110: \mathrm{HR}: 0.72,95 \% \mathrm{Cl}: 0.42,1.22\end{array}$ & $\begin{array}{l}\text { Sex, age, alcohol intake, smoking, } \\
\text { asbestos exposure } \\
\text { Other: }\end{array}$ \\
\hline
\end{tabular}

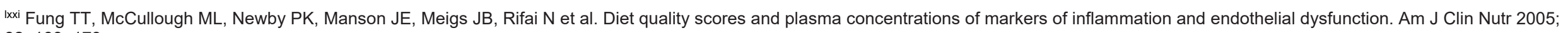

82: 163-173. 


\section{Study and \\ Participant \\ Characteristics \\ Analytic N: 4336 \\ Participants were $34 \%$ female, $>50$ yo, $53 \%$ overweight or obese, $100 \%$ heavy smokers (80\% current) \\ Excluded those with prevalent cancer, $<50 y$ of age, not current smokers or had quit smoking $>10 y$ ago and smoked $<20$ pack years, did not return FFQ, abnormal total energy intake $(\sim 17 \%$ of original sample)}

Results
Intervention/Exposure and Outcomes
- Positive components: Vegetables (not potatoes), legumes, fruits, nuts, whole grains, fish,

MUFA/SFAs

- 5-7, n=72: HR: 0.61, 95\% Cl: 0.34, 1.12

- 8-9, n=2: HR: $0.20,95 \%$ Cl: 0.04, 0.91

- $p$-trend $=0.04$

- Neutral components: Alcohol

- Negative components: Red and

\section{Non-Significant: N/A}

Dietary assessment methods: 188-item, validated FFQ at baseline, age $>50 y$

patient screening, medical records confirmed by histology or cytology processed meat

\section{Outcome assessment methods: Annual}

Confounding, Study Limitations, and Summary of Findings

Total energy intake, dietary inflammatory index score

\section{Limitations:}

- Did not account for race/ethnicity, socioeconomic status, physical activity, anthropometry, family history of the cancer outcome, lung disease

- Exposure occurred prior to start of $\mathrm{f} / \mathrm{u}$

- Only assessed dietary intake once at baseline; did not account for possible changes in dietary intake over f/u

- Did not account for missing data

- No preregistered statistical plan; potential for selective outcome reporting

Funding Sources: Italian Association for Cancer Research, Italian Foundation for Cancer Research, European Institute of Oncology, $\mathrm{NCl}$

Summary: Higher aMED score at $>50 y$ was associated with significantly lower risk of lung cancer after $8.5 \mathrm{y} / \mathrm{f}$.

\section{Schulpen, $2018^{48}$ \\ Study (Netherlands}

\section{Dietary patterns:}

- alternate Mediterranean diet scores $\left(\right.$ aMED) ${ }^{1 \times x i i}$, categorical (tertiles) and continuous (per 2 pt increment), and without alcohol (aMEDr)

\section{Significant:}

\section{Key confounders accounted for:}

Men, WCRF/AICR score at $61 \mathrm{y}$, including alcohol, per SD increment and lung cancer after $20.3 \mathrm{y} / \mathrm{u}$ :

HR: $0.90,95 \% \mathrm{Cl}: 0.83,0.98$
Sex, age, education, alcohol intake, history of the cancer, lung disease physical activity, smoking, BMI, family

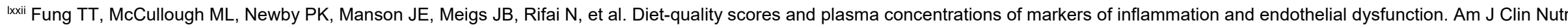
2005;82: 163-73. 


\begin{tabular}{|c|c|c|c|}
\hline $\begin{array}{l}\text { Study and } \\
\text { Participant } \\
\text { Characteristics }\end{array}$ & Intervention/Exposure and Outcomes & Results & $\begin{array}{l}\text { Confounding, Study Limitations, and } \\
\text { Summary of Findings }\end{array}$ \\
\hline $\begin{array}{l}\text { Analytic N: } 6581 \\
\text { Participants were } 52 \% \\
\text { female, } \sim 61 \text { yo ( } 55- \\
69 \mathrm{y}), \sim 24.9 \mathrm{~kg} / \mathrm{m}^{2} \mathrm{BMI}, \\
\sim 56 \% \text { never-smokers; } \\
\sim 9.7 \mathrm{~g} / \mathrm{d} \text { alcohol, } 61.8 \\
\mathrm{~min} / \mathrm{d} \text { non- } \\
\text { occupational physical } \\
\text { activity, } 9.7 \% \text { family } \\
\text { history of lung cancer }\end{array}$ & $\begin{array}{l}\text { Positive components: Vegetables } \\
\text { (not potatoes), legumes, fruits, } \\
\text { nuts, whole grains, fish, } \\
\text { MUFA/SFAs } \\
\circ \quad \text { Neutral components: Alcohol } \\
\text { Negative components: Red and } \\
\text { processed meat } \\
\text { modified Mediterranean diet scores } \\
\text { (mMED) }{ }^{\mid x x i i i} \text {, categorical (tertiles) and } \\
\text { continuous (per } 2 \text { pt increment), and } \\
\text { without alcohol (mMEDr) } \\
\circ \quad \text { Positive components: Vegetables, } \\
\text { legumes, fruit, cereals, fish, } \\
\text { MUFA+PUFA/SFA } \\
\text { Neutral components: Alcohol } \\
\circ \quad \text { Negative components: Meat, } \\
\text { dairy products } \\
\text { WCRF/AICR score } \\
\text { continiv, diet only, } \\
\text { without alcoho (per SD increment), and } \\
\circ \quad \text { Positive components: Vegetables } \\
\text { and fruit, dietary fiber } \\
\circ \quad \text { Negative components: Red and } \\
\text { processed meat, sugary drinks, } \\
\text { alcohol, sodium, energy-dense } \\
\text { foods }\end{array}$ & 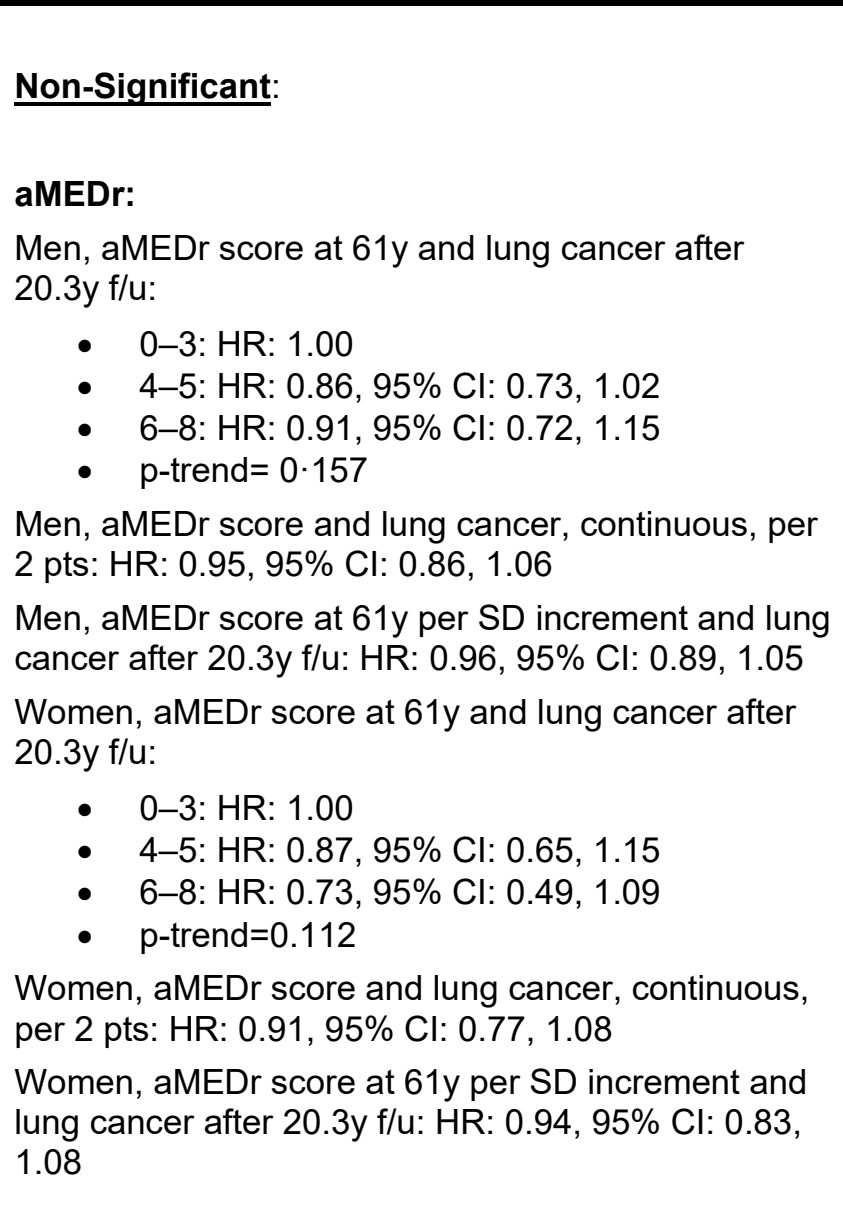 & $\begin{array}{l}\text { Other: } \\
\text { Daily energy intake } \\
\text { Limitations: } \\
\text { - } \quad \text { Did not account for race/ethnicity, } \\
\text { environmental exposures to lung } \\
\text { carcinogens } \\
\text { - } \quad \text { Exposure occurred prior to start of f/u } \\
\text { - Only assessed dietary intake once at } \\
\text { baseline; did not account for possible } \\
\text { changes in dietary intake over f/u } \\
\text { - Did not account for missing data } \\
\text { - } \quad \text { No preregistered statistical plan; } \\
\text { serious potential for selective } \\
\text { outcome reporting }\end{array}$ \\
\hline
\end{tabular}

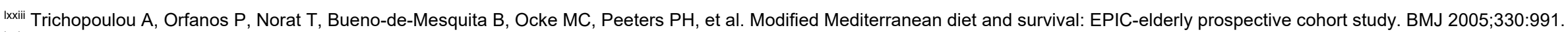

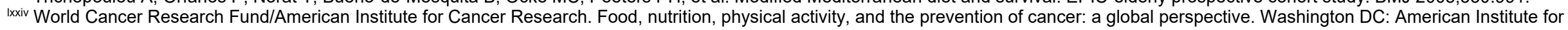
Cancer Research; 2007. 


\section{Intervention/Exposure and Outcomes}

Results

aMED:

Outcome assessment methods:

Netherlands Cancer Registry and the nationwide

Dutch Pathology Registry, and review of pathology records f/u:
Men, aMED score at $61 \mathrm{y}$ and lung cancer after $20.3 \mathrm{y}$

- 0-3: HR: 1.00

- 4-5: HR: $0.86,95 \% \mathrm{Cl}: 0.72,1.03$

- 6-8: HR: $0.89,95 \%$ Cl: 0.72, 1.10

- $p$-trend $=0.177$

Men, aMED score and lung cancer, continuous, per 2 pts: HR: 0.96, 95\% Cl: 0.87, 1.05

Men, aMED score at 61y per SD increment and lung cancer after 20.3y f/u: HR: 0.97, 95\% Cl: 0.89, 1.05

Women, aMED score at $61 \mathrm{y}$ and lung cancer after 20.3y f/u:

- 0-3: HR: 1.00

- 4-5: HR: $1.03,95 \%$ Cl: 0.77, 1.39

- 6-8: HR: $0.80,95 \%$ Cl: $0.55,1.15$

- $p$-trend $=0.326$

Women, aMED score and lung cancer, continuous, per 2 pts: HR: 0.93, 95\% Cl: 0.79, 1.08

Women, aMED score at 61y per SD increment and lung cancer after $20.3 \mathrm{y}$ f/u: HR: 0.94, 95\% Cl: 0.83, 1.07

\section{mMEDr:}

Men, mMEDr score at $61 \mathrm{y}$ and lung cancer after 20.3y f/u:

- 0-3: HR: 1.00

- 4-5: HR: 1.11, 95\% Cl: 0.93, 1.32

- 6-8: HR: $0.96,95 \% \mathrm{Cl}: 0.76,1.21$
Confounding, Study Limitations, and Summary of Findings 
- $\quad$-trend $=0.901$

Men, mMEDr score and lung cancer, continuous, per 2 pts: HR: 1.00, 95\% Cl: 0.90, 1.11

Women, mMEDr score at $61 \mathrm{y}$ and lung cancer after 20.3y f/u:

- 0-3: HR: 1.00

- 4-5: HR: $0.99,95 \%$ Cl: $0.75,1.32$

- 6-8: HR: 0.83, 95\% Cl: 0.56, 1.24

- $p$-trend $=0.474$

Women, mMEDr score and lung cancer, continuous, per 2 pts: HR: $0.94,95 \% \mathrm{Cl}: 0.79,1.11$

\section{MMED:}

Men, mMED score at $61 \mathrm{y}$ and lung cancer after 20.3y f/u:

- 0-3: HR: 1.00

- 4-5: HR: $0.99,95 \%$ Cl: $0.82,1.19$

- 6-8: HR: 0.98, 95\% Cl: 0.78, 1.21

- $p$-trend $=0.823$

Men, mMED score and lung cancer, continuous, per 2 pts: HR: $1.02,95 \%$ Cl: 0.92, 1.12

Women, mMED score at $61 \mathrm{y}$ and lung cancer after 20.3y f/u:

- 0-3: HR: 1.00

- 4-5: HR: $0.83,95 \% \mathrm{Cl}: 0.61,1.11$

- 6-8: HR: 0.87, 95\% Cl: 0.61, 1.25

- $p$-trend $=0.339$

Women, mMED score and lung cancer, continuous, per 2 pts: HR: $0.94,95 \% \mathrm{Cl}: 0.80,1.11$ 
All results were similar when the lung cancer substypes were examined, including adenocarcinoma, squamous cell, small cell carcinoma, and large cell carcinoma.

\section{WCRF/AICR, diet only:}

Men, WCRF/AICR score at 61y, excluding alcohol, per SD increment and lung cancer after 20.3y f/u: HR: 0.96, 95\% Cl: 0.89, 1.05

Women, WCRF/AICR score at 61y, excluding alcohol, per SD increment and lung cancer after 20.3y f/u: HR: 0.94, 95\% Cl: 0.83, 1.08

Women, WCRF/AICR score, including alcohol, at $61 \mathrm{y}$ per SD increment and lung cancer after $20.3 \mathrm{y} / \mathrm{u}$ : HR: $0.94,95 \% \mathrm{Cl}: 0.81,1.08$

\begin{tabular}{|c|c|c|}
\hline Voortman, $2017^{24}$ & \multirow{2}{*}{\multicolumn{2}{|c|}{$\begin{array}{l}\text { Dietary patterns: } \\
\text { - Dutch Dietary Guidelines } 2015 \text { score, } \\
\text { continuous }\end{array}$}} \\
\hline $\begin{array}{l}\text { PCS (Rotterdam } \\
\text { Studv) }\end{array}$ & & \\
\hline The Netherlands & ० & \\
\hline Analytic N: 9619 & & $\begin{array}{l}\text { fish, dairy products, unsaturated } \\
\text { fats and oils, tea }\end{array}$ \\
\hline $\begin{array}{l}\text { Participants were } 58 \% \\
\text { female, } 64.1 \text { yo, } 49.0- \\
82.8 \text { yo, } 26.3 \mathrm{~kg} / \mathrm{m}^{2} \\
\text { BMI. } 32 \% \text { never }\end{array}$ & ○ & $\begin{array}{l}\text { Negative components: Replace } \\
\text { refined grains with whole-grain } \\
\text { products, red meat, processed } \\
\text { meat, alcohol, sodium }\end{array}$ \\
\hline
\end{tabular}
smokers, $61 \%<10 \mathrm{~g} / \mathrm{d}$ alcohol, $15.5 \%$ higher education

Dietary assessment methods: 170 to

\section{Significant: N/A}

\section{$\underline{\text { Non-Significant: }}$}

Dutch Dietary Guidelines 2015 score and lung cancer $(n=204)$ after $11.1 \mathrm{y}$ f/u: HR: $0.93,95 \% \mathrm{Cl}$ $0.86,1.01$ 389-item, validated FFQ at baseline, $64 \mathrm{y}$

\section{Key confounders accounted for:}

Sex, age, education, employment status, alcohol intake, physical activity, smoking, BMI

Other:

Cohort, total energy intake

\section{Limitations:}

- Did not account for race/ethnicity, family history of the cancer outcome, lung disease, environmental exposures to lung carcinogens

- Exposure occurred prior to start of $\mathrm{f} / \mathrm{u}$

- Only assessed dietary intake once at baseline; did not account for possible changes in dietary intake over f/u 


\section{Study and}

Participant

Characteristics

Intervention/Exposure and Outcomes

Results

\section{Outcome assessment methods:}

Excluded those

without reliable dietary

data, prevalent cancer

cases, those who

nationwide registry of histopathology and

cytopathology, f/u with general

developed disease

within 2 y of baseline

missing outcome data

( $19 \%$ of original

sample) practitioners
Confounding, Study Limitations, and Summary of Findings

- Did not account for missing data

- No preregistered statistical plan; potential for selective outcome reporting

Funding Sources: Erasmus University Medical Center and Erasmus University Rotterdam; the Netherlands Organization for Health Research and Development; the Research Institute for Diseases in the Elderly; the Netherlands Genomics Initiative;

the Ministry of Education, Culture and Science; the Ministry of Health, Welfare and Sports; the European Commission (DG XII); and the Municipality of Rotterdam

Summary: Dutch Dietary Guidelines 2015 score at $64 y$ was not significantly associated with lung cancer after $11 y \mathrm{f} / \mathrm{u}$. 
Table 10. Summary of the results from studies that examined the relationship between dietary patterns and lung cancerlxxv

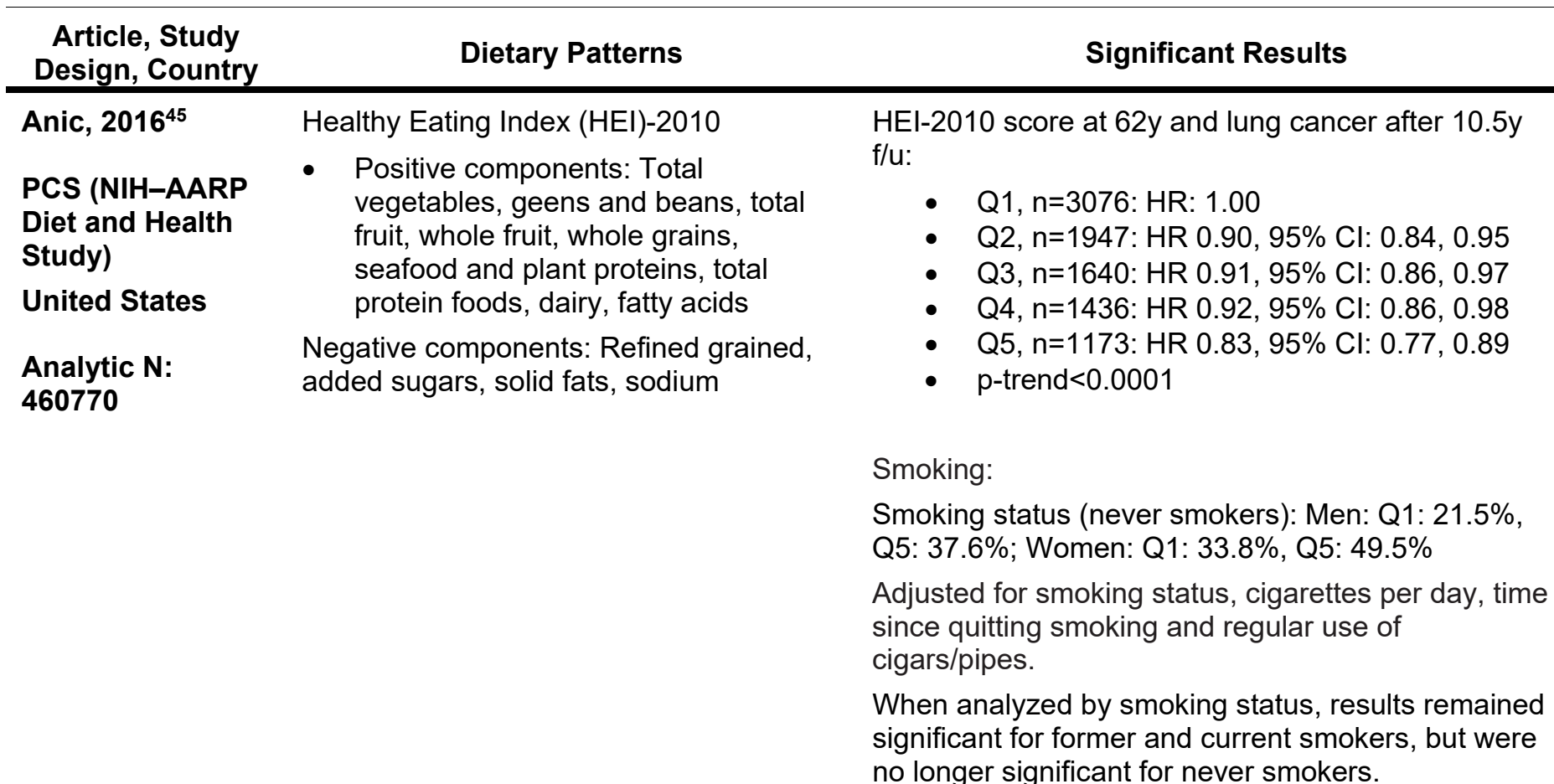

Ixxv Abbreviations: AHEI-2010, Alternative Healthy Eating Index-2010; aMED, Alternative Mediterranean Diet Score ; BMI, body mass index; d, day; DASH, Dietary Approaches to Stop Hypertension; DP, Dietary pattern; \%E, \% of energy; EPIC, European Prospective Investigation into Cancer and Nutrition; FFQ, food frequency questionnaire; FSAm-NPS, Nutrient Profiling System of the British Food Standards Agency (modified version); f/u, follow-up; HR, hazard ratio; MDS, Mediterranean Diet Score; mMEDr, modified Mediterranean diet scores; mMEDr, modified Mediterranean diet scores without alcohol; mo, month or months; N/A, Not applicable; NIH, National Institutes of Health; NS, Not significant; NR, Not reported; PCS, prospective cohort study; pt, point; RCT, randomized controlled trial; WCRF/AICR, World Cancer Research Fund/American Institute for Cancer Research; wk, weeks; y, year 


\begin{tabular}{|c|c|c|c|}
\hline $\begin{array}{l}\text { Article, Study } \\
\text { Design, Country }\end{array}$ & Dietary Patterns & Significant Results & Non-Significant Results \\
\hline & $\begin{array}{l}\text { Alternative Healthy Eating Index (AHEI)- } \\
2010\end{array}$ & $\begin{array}{l}\text { AHEI-2010 score at } 62 y \text { and lung cancer after } 10.5 y \\
\text { f/u: }\end{array}$ & \\
\hline & $\begin{array}{l}\text { - Positive components: Vegetables } \\
\text { (not potatoes, French fries), Fruit, } \\
\text { Whole Grains, Long-Chain Fats (EPA } \\
\text { + DHA), PUFA } \\
\text { - } \quad \text { Neutral components: Alcohol } \\
\text { - Negative components: Red and } \\
\text { Processed Meat, Sugar Sweetened }\end{array}$ & 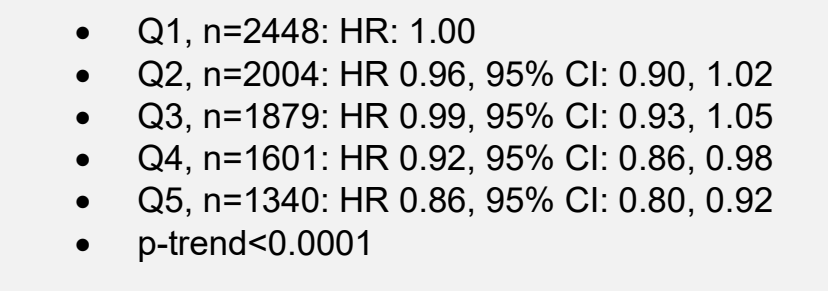 & \\
\hline & $\begin{array}{l}\text { Beverages and Fruit Juice, Trans FA, } \\
\text { Sodium }\end{array}$ & Smoking: & \\
\hline & & $\begin{array}{l}\text { Smoking status (never smokers): Men: Q1: } 27.6 \% \text {, } \\
\text { Q5: 31.9\%; Women: Q1: } 44.9 \% \text {, Q5: } 40.6 \%\end{array}$ & \\
\hline & & $\begin{array}{l}\text { Adjusted for smoking status, cigarettes per day, time } \\
\text { since quitting smoking and regular use of } \\
\text { cigars/pipes. }\end{array}$ & \\
\hline & & $\begin{array}{l}\text { When analyzed by smoking status, results remained } \\
\text { significant for former smokers, but were no longer } \\
\text { significant for never or current smokers. }\end{array}$ & \\
\hline
\end{tabular}


Article, Study Design, Country

Alternative Mediterranean Diet Score (aMED)

- Positive components: Vegetables (not potatoes), legumes, fruits, nuts, whole grains, fish, MUFA/SFAs

- Neutral components: Alcohol

Negative components: Red and processed meat
aMED score at $62 \mathrm{y}$ and lung cancer after $10.5 \mathrm{y} / \mathrm{u}$ :

- Q1, n=2232: HR: 1.00

- Q2, n=1849: HR 0.96, 95\% Cl: 0.91, 1.03

- Q3, $n=1890:$ HR $0.94,95 \%$ Cl: $0.88,1.00$

- Q4, n=1660: HR 0.96, 95\% Cl: 0.90, 1.03

- Q5, $n=1641:$ HR 0.85, 95\% Cl: 0.79, 0.91

- $\mathrm{p}$-trend $<0.0001$

Smoking:

Smoking status (never smokers): Men: Q1: 26.8\%, Q5: 33.4\%; Women: Q1: 41.4\%, Q5: 46.4\%

Adjusted for smoking status, cigarettes per day, time since quitting smoking and regular use of

cigars/pipes.

When analyzed by smoking status, results remained significant for former smokers, but were no longer significant for never or current smokers. 


\begin{tabular}{|c|c|c|c|}
\hline $\begin{array}{l}\text { Article, Study } \\
\text { Design, Country }\end{array}$ & Dietary Patterns & Significant Results & Non-Significant Results \\
\hline & $\begin{array}{l}\text { DASH Score } \\
\text { Positive components: Vegetables } \\
\text { (not potatoes), nuts and legumes, } \\
\quad \text { fruit and fruit juice, whole grains, low- } \\
\quad \text { fat dairy } \\
\text { Negative components: Red and } \\
\text { processed meat, sweetened beverages, } \\
\text { sodium }\end{array}$ & 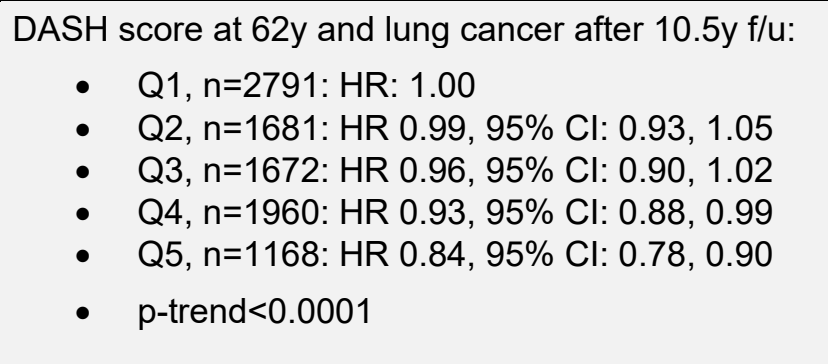 & \\
\hline & & Smoking: & \\
\hline & & $\begin{array}{l}\text { Smoking status (never smokers): Men: Q1: } 24.5 \% \text {, } \\
\text { Q5: 36.1\%; Women: Q1: } 39.3 \% \text {, Q5: } 48.0 \%\end{array}$ & \\
\hline & & $\begin{array}{l}\text { Adjusted for smoking status, cigarettes per day, time } \\
\text { since quitting smoking and regular use of } \\
\text { cigars/pipes. }\end{array}$ & \\
\hline & & $\begin{array}{l}\text { When analyzed by smoking status, results remained } \\
\text { significant for former smokers, but were no longer } \\
\text { significant for never or current smokers. }\end{array}$ & \\
\hline
\end{tabular}


Article, Study

Design, Country

Boden, 201927

PCS

(Vasterbotten

Intervention

Programme)

Sweden

Analytic N: 35393
Dietary Patterns

Mediterranean diet score (MDS)

- Positive components: Vegetables and potatoes, fruit and fresh juices, wholegrain cereals, fish and fish products, MUFA+PUFA/SFA,

- Moderation components: Alcohol

- Negative components: Meat and meat products, dairy products
Significant Results

Non-Significant Results

All participants, MDS score, per tertile increase, at $46 y$ and lung cancer $(n=442$ 15y f/u: HR: 0.90, 95\% Cl: 0.80, 1.01

Men, MDS score, per tertile increase, at and lung cancer $(n=210)$ after 15y f/u: H $0.86,95 \% \mathrm{Cl}: 0.72,1.03$

Women, MDS score, per tertile increase, $46 y$ and lung cancer $(n=232)$ after $15 \mathrm{y} / \mathrm{l}$ HR: $0.94,95 \% \mathrm{Cl}: 0.79,1.10$

Smoking: Smoking status (\% current smokers): Me
T1: 16.8, T2: 15.3, T3: 12.7; Women: T1 19.6, T2: 17.4, T3: 15.2

Adjusted for smoking status (non smoker ever smokers) 


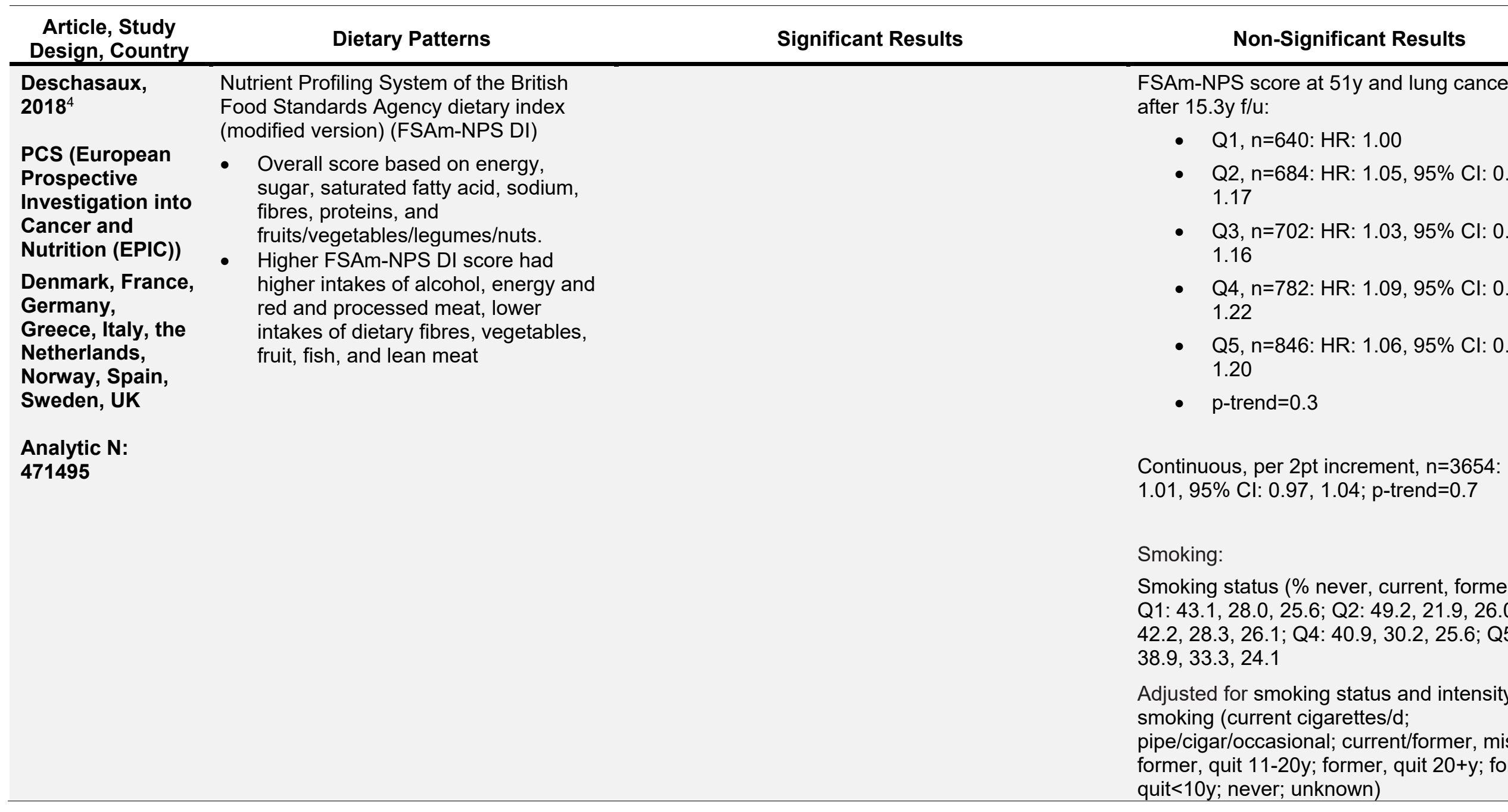




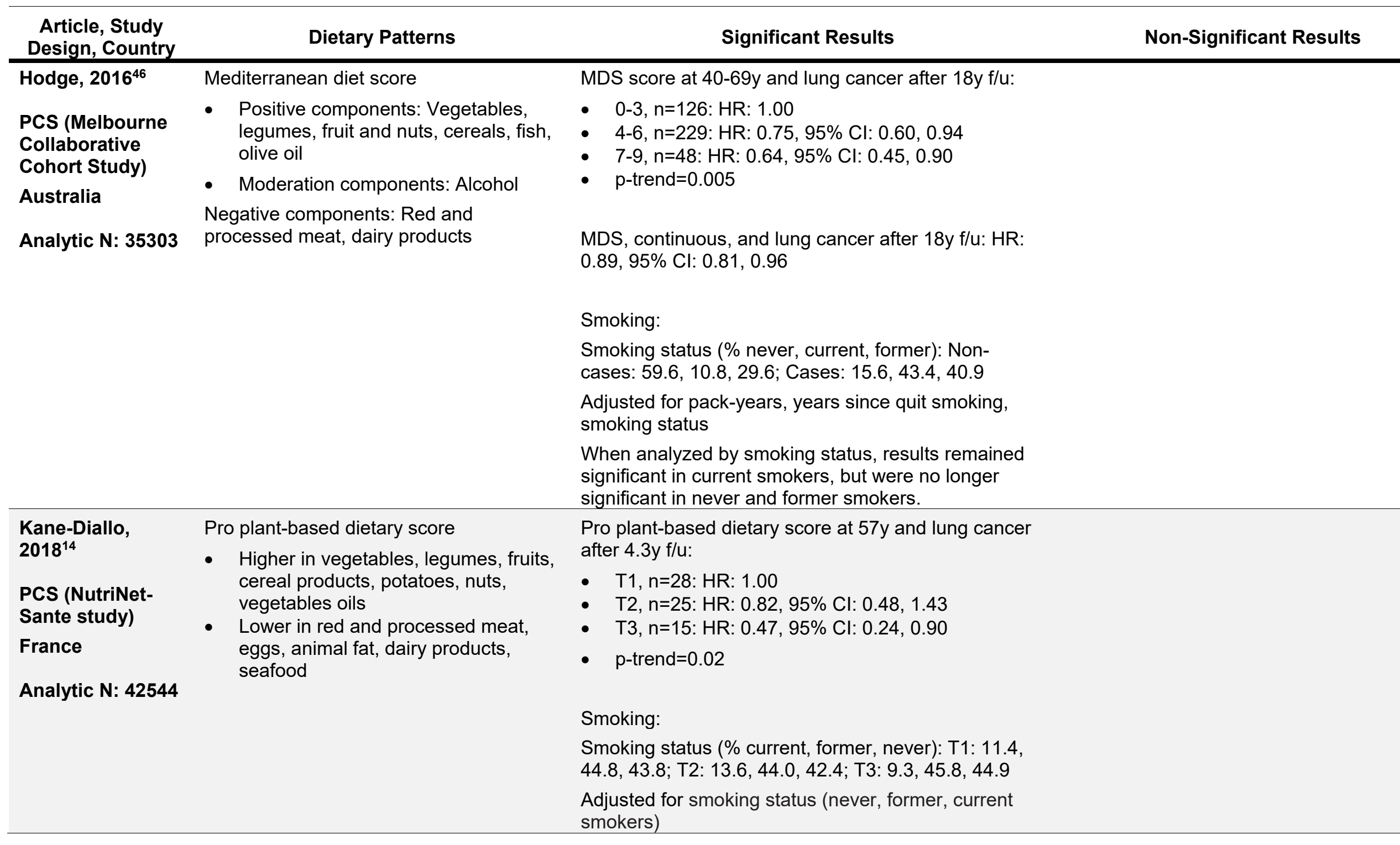


Article, Study Design, Country

Maisonneuve

$2016^{47}$

\section{PCS (COSMOS} study)

Italy

Analytic N: 4336
Alternative Mediterranean Diet Score (aMED)

- Positive components: Vegetables (not potatoes), legumes, fruits, nuts, whole grains, fish, MUFA/SFAs

- Neutral components: Alcohol

- Negative components: Red and processed meat
aMED score at $>50 \mathrm{y}$ and lung cancer after $8.5 \mathrm{y} / \mathrm{u}$ :

- 0-1, n=16: HR: 1.00

- 2-4, n=110: HR: 0.72, 95\% Cl: 0.42, 1.22

- 5-7, n=72: HR: 0.61, 95\% Cl: 0.34, 1.12

- 8-9, n=2: HR: 0.20, 95\% Cl: 0.04, 0.91

p-trend $=0.04$

Smoking:

Smoking: $100 \%$ heavy smokers $(20.2 \%$ former,

$79.8 \%$ current; excluded those who were not current smokers or had quit smoking $>10 \mathrm{y}$ ago and smoked $<20$ pack years)

Adjusted for smoking duration, smoking intensity, and years of smoking cessation 


\begin{tabular}{|c|c|c|c|}
\hline $\begin{array}{c}\text { Article, Study } \\
\text { Design, Country }\end{array}$ & Dietary Patterns & Significant Results & Non-Significant Results \\
\hline Schulpen, $2018^{48}$ & $\begin{array}{l}\text { alternate Mediterranean diet score } \\
\text { (aMED) }\end{array}$ & & $\begin{array}{l}\text { Men, aMED score at } 61 \mathrm{y} \text { and lung cance } \\
\text { after } 20.3 \mathrm{y} \text { f/u: }\end{array}$ \\
\hline $\begin{array}{l}\text { Nested Case- } \\
\text { Control Study } \\
\text { (Netherlands }\end{array}$ & $\begin{array}{l}\text { Positive components: Vegetables } \\
\text { (not potatoes), legumes, fruits, nuts, } \\
\text { whole grains, fish, MUFA/SFAs }\end{array}$ & & $\begin{array}{l}\text { - } \quad \text { 4-3: HR: } 1.00 \\
\text { 4-5: HR: } 0.86,95 \% \mathrm{Cl}: 0.72,1.0 \\
\text { 6-8: HR: } 0.89,95 \% \text { Cl: } 0.72,1.1\end{array}$ \\
\hline Cohort Study & - Neutral components: Alcohol & & - $\quad p$-trend $=0.177$ \\
\hline $\begin{array}{l}(\text { NLCS)) } \\
\text { The Netherlands }\end{array}$ & $\begin{array}{l}\text { - Negative components: Red and } \\
\text { processed meat }\end{array}$ & & $\begin{array}{l}\text { Men, aMED score and lung cancer, } \\
\text { continuous, per } 2 \text { pts: HR: } 0.96,95 \% \mathrm{Cl} \text { : } \\
1.05\end{array}$ \\
\hline \multirow[t]{8}{*}{ Analytic N: 6581} & & & $\begin{array}{l}\text { Men, aMED score at } 61 \mathrm{y} \text { per SD increme } \\
\text { and lung cancer after } 20.3 \mathrm{y} \text { f/u: HR: } 0.97 \\
95 \% \mathrm{Cl}: 0.89,1.05\end{array}$ \\
\hline & & & $\begin{array}{l}\text { Women, aMED score at } 61 \mathrm{y} \text { and lung ca } \\
\text { after } 20.3 \mathrm{y} / \mathrm{u} \text { : }\end{array}$ \\
\hline & & & $\begin{array}{l}\text { - } \quad \text { 0-3: HR: } 1.00 \\
\text { 4-5: HR: } 1.03,95 \% \mathrm{Cl}: 0.77,1.3 \\
\text { - } \quad \text {-8: HR: } 0.80,95 \% \mathrm{Cl}: 0.55,1.1 \\
\text { p-trend }=0.326\end{array}$ \\
\hline & & & $\begin{array}{l}\text { Women, aMED score and lung cancer, } \\
\text { continuous, per } 2 \text { pts: HR: } 0.93,95 \% \mathrm{Cl} \text { : } \\
1.08\end{array}$ \\
\hline & & & $\begin{array}{l}\text { Women, aMED score at } 61 \mathrm{y} \text { per SD } \\
\text { increment and lung cancer after } 20.3 \mathrm{y} / \mathrm{\iota} \\
0.94,95 \% \mathrm{Cl}: 0.83,1.07\end{array}$ \\
\hline & & & Smoking: \\
\hline & & & $\begin{array}{l}\text { Smoking ( } \% \text { never, former): NR by aMED } \\
\text { score }\end{array}$ \\
\hline & & & $\begin{array}{l}\text { Adjusted for smoking status (never, form } \\
\text { current), cigarette smoking duration (yea } \\
\text { centred) and cigarette smoking frequenc } \\
\text { (cigarettes smoked per day, centred) }\end{array}$ \\
\hline
\end{tabular}


\begin{tabular}{ccc}
$\begin{array}{c}\text { Article, Study } \\
\text { Design, Country }\end{array} \quad$ Dietary Patterns & Significant Results \\
\hline
\end{tabular}

aMED without alcohol (aMEDr)

\section{Non-Significant Results}

Men, aMEDr score at $61 \mathrm{y}$ and lung canc after 20.3y f/u:

- 0-3: HR: 1.00

- 4-5: HR: 0.86, 95\% Cl: 0.73, 1.0

- 6-8: HR: 0.91, 95\% Cl: 0.72, 1.1

- $p$-trend $=0.157$

Men, aMEDr score and lung cancer, continuous, per 2 pts: HR: $0.95,95 \% \mathrm{Cl}$ : 1.06

Men, aMEDr score at 61y per SD increm and lung cancer after 20.3y f/u: HR: 0.96 95\% Cl: 0.89, 1.05

Women, aMEDr score at $61 \mathrm{y}$ and lung cé after 20.3y f/u:

- 0-3: HR: 1.00

- 4-5: HR: 0.87, 95\% Cl: 0.65, 1.1

- 6-8: HR: 0.73, 95\% Cl: 0.49, 1.0

- $p$-trend $=0.112$

Women, aMEDr score and lung cancer, continuous, per 2 pts: HR: $0.91,95 \% \mathrm{Cl}$ : 1.08

Women, aMEDr score at 61y per SD increment and lung cancer after $20.3 \mathrm{y} / \mathrm{c}$ $0.94,95 \% \mathrm{Cl}: 0.83,1.08$

Smoking:

Smoking (\% never, former): 0-3: 11.5, 48 5: 13.1, 52.4; 6-8: 15.9, 62.2

Adjusted for smoking status (never, form current), cigarette smoking duration (yea centred) and cigarette smoking frequenc (cigarettes smoked per day, centred) 


\begin{tabular}{|c|c|c|c|}
\hline $\begin{array}{l}\text { Article, Study } \\
\text { Design, Country }\end{array}$ & Dietary Patterns & Significant Results & Non-Significant Results \\
\hline & $\begin{array}{l}\text { modified Mediterranean diet score } \\
\text { (mMED) }\end{array}$ & & $\begin{array}{l}\text { Men, mMED score at } 61 \mathrm{y} \text { and lung cance } \\
\text { after } 20.3 \mathrm{y} / \mathrm{u} \text { : }\end{array}$ \\
\hline & \multirow{9}{*}{$\begin{array}{l}\text { Positive components: Vegetables, } \\
\text { legumes, fruit, cereals, fish, } \\
\text { MUFA+PUFA/SFA } \\
\text { - Neutral components: Alcohol } \\
\text { - Negative components: Meat, dairy } \\
\text { products }\end{array}$} & & $\begin{array}{l}\text { - } \quad \text { 0-3: HR: } 1.00 \\
\text { 4-5: HR: } 0.99,95 \% \mathrm{Cl}: 0.82,1.1 \\
\text { - } \quad \text { 6-8: HR: } 0.98,95 \% \mathrm{Cl}: 0.78,1.2 \\
\text { - p-trend }=0.823\end{array}$ \\
\hline & & & $\begin{array}{l}\text { Men, mMED score and lung cancer, } \\
\text { continuous, per } 2 \text { pts: HR: } 1.02,95 \% \mathrm{Cl} \text { : } \\
1.12\end{array}$ \\
\hline & & & $\begin{array}{l}\text { Women, mMED score at } 61 \mathrm{y} \text { and lung ce } \\
\text { after } 20.3 \mathrm{y} \text { f/u: }\end{array}$ \\
\hline & & & $\begin{array}{ll}- & \text { 0-3: HR: } 1.00 \\
\text { - 4-5: HR: } 0.83,95 \% \mathrm{Cl}: 0.61,1.1 \\
\text { 6-8: HR: } 0.87,95 \% \mathrm{Cl}: 0.61,1.2 \\
\text { - p-trend }=0.339\end{array}$ \\
\hline & & & $\begin{array}{l}\text { Women, mMED score and lung cancer, } \\
\text { continuous, per } 2 \text { pts: HR: } 0.94,95 \% \mathrm{Cl} \text { : } \\
1.11\end{array}$ \\
\hline & & & $\begin{array}{l}\text { All results were similar when the lung car } \\
\text { substypes were examined, including } \\
\text { adenocarcinoma, squamous cell, small c } \\
\text { carcinoma, and large cell carcinoma. }\end{array}$ \\
\hline & & & Smoking: \\
\hline & & & $\begin{array}{l}\text { Smoking (\% never, former): NR by mMEl } \\
\text { score }\end{array}$ \\
\hline & & & $\begin{array}{l}\text { Adjusted for smoking status (never, form } \\
\text { current), cigarette smoking duration (yea } \\
\text { centred) and cigarette smoking frequenc } \\
\text { (cigarettes smoked per day, centre) }\end{array}$ \\
\hline
\end{tabular}


Article, Study

Design, Country

Men, mMEDr score at $61 \mathrm{y}$ and lung canc after 20.3y f/u:

- 0-3: HR: 1.00

- 4-5: HR: $1.11,95 \% \mathrm{Cl}: 0.93,13$

- 6-8: HR: 0.96, 95\% Cl: 0.76, 1.2

- $p$-trend $=0.901$

Men, mMEDr score and lung cancer, continuous, per 2 pts: HR: $1.00,95 \% \mathrm{Cl}$ : 1.11

Women, mMEDr score at 61y and lung c after 20.3y f/u:

- 0-3: HR: 1.00

- 4-5: HR: 0.99, 95\% Cl: $0.75,1.3$

- 6-8: HR: 0.83, 95\% Cl: 0.56, 1.2

- $p$-trend $=0.474$

Women, mMEDr score and lung cancer, continuous, per 2 pts: HR: 0.94, 95\% Cl: 1.11

\section{Smoking:}

Smoking (\% never, former): 0-3: 11.5, 48 75: 14.7, 51.7; 6-8: 10.3, 61.6

Adjusted for smoking status (never, form current), cigarette smoking duration (yea centred) and cigarette smoking frequenc (cigarettes smoked per day, centre) 


\begin{tabular}{|c|c|c|c|}
\hline $\begin{array}{l}\text { Article, Study } \\
\text { Design, Country }\end{array}$ & Dietary Patterns & Significant Results & Non-Significant Results \\
\hline & \multirow{5}{*}{\multicolumn{2}{|c|}{$\begin{array}{l}\text { WCRF/AICR score, diet only } \\
\text { - Positive components: Vegetables } \\
\text { and fruit, dietary fiber } \\
\text { - Negative components: Red and } \\
\text { processed meat, sugary drinks, } \\
\text { alcohol, sodium, energy-dense foods }\end{array}$}} & $\begin{array}{l}\text { Men, WCRF/AICR score at } 61 \mathrm{y} \text {, includin } \\
\text { alcohol, per SD increment and lung canc } \\
\text { after } 20.3 \mathrm{y} / \mathrm{u} \text { : HR: } 0.90,95 \% \mathrm{Cl}: 0.83, \mathrm{C}\end{array}$ \\
\hline & & & $\begin{array}{l}\text { Women, WCRF/AICR score, including } \\
\text { alcohol, at } 61 \mathrm{y} \text { per SD increment and lur } \\
\text { cancer after } 20.3 \mathrm{y} \text { f/u: HR: } 0.94,95 \% \mathrm{Cl} \\
1.08\end{array}$ \\
\hline & & & Smoking: \\
\hline & & & $\begin{array}{l}\text { Smoking (\% never, former): NR by } \\
\text { WCRF/AICR, diet only score }\end{array}$ \\
\hline & & & $\begin{array}{l}\text { Adjusted for smoking status (never, form } \\
\text { current), cigarette smoking duration (yea } \\
\text { centred) and cigarette smoking frequenc } \\
\text { (cigarettes smoked per day, centred) }\end{array}$ \\
\hline & \multirow[t]{5}{*}{$\begin{array}{l}\text { WCRF/AICR score, diet only, without } \\
\text { alcohol }\end{array}$} & & $\begin{array}{l}\text { Men, WCRF/AICR score at } 61 \mathrm{y} \text {, without } \\
\text { alcohol, per SD increment and lung canc } \\
\text { after } 20.3 \mathrm{y} / \mathrm{u}: \mathrm{HR}: 0.96,95 \% \mathrm{Cl}: 0.89,1\end{array}$ \\
\hline & & & $\begin{array}{l}\text { Women, WCRF/AICR score at } 61 \mathrm{y} \text {, with } \\
\text { alcohol, per SD increment and lung canc } \\
\text { after } 20.3 \mathrm{y} / \mathrm{u}: \mathrm{HR}: 0.94,95 \% \mathrm{Cl}: 0.83,1\end{array}$ \\
\hline & & & Smoking: \\
\hline & & & $\begin{array}{l}\text { Smoking (\% never, former): NR by } \\
\text { WCRF/AICR score, diet only, without alc }\end{array}$ \\
\hline & & & $\begin{array}{l}\text { Adjusted for smoking status (never, form } \\
\text { current), cigarette smoking duration (yea } \\
\text { centred) and cigarette smoking frequenc } \\
\text { (cigarettes smoked per day, centred) }\end{array}$ \\
\hline
\end{tabular}


Article, Study

Design, Country

Voortman, 201724

PCS (Rotterdam

Study)

The Netherlands

Analytic N: 9619
Dietary Patterns

Significant Results

Dutch Dietary Guidelines 2015 score

- Positive components: Vegetables, legumes, fruit, nuts, whole grains, fish, dairy products, unsaturated fats and oils, tea

Negative components: Replace refined grains with whole-grain products, red meat, processed meat, alcohol, sodium
Non-Significant Results

Dutch Dietary Guidelines 2015 score anc cancer $(n=204)$ after 11.1y f/u: HR: 0.93, Cl: $0.86,1.01$

Smoking:

Smoking status (\% never, ever, current): 44.2, 23.8

Adjusted for smoking status

Table 11. Risk of bias for observational studies examining dietary patterns and lung cancerlxxvi

\begin{tabular}{|c|c|c|c|c|c|c|c|}
\hline & Confounding & $\begin{array}{l}\text { Selection of } \\
\text { participants }\end{array}$ & $\begin{array}{l}\text { Classification } \\
\text { of exposures }\end{array}$ & $\begin{array}{c}\text { Deviations } \\
\text { from intended } \\
\text { exposures }\end{array}$ & Missing data & $\begin{array}{c}\text { Outcome } \\
\text { measurement }\end{array}$ & $\begin{array}{l}\text { Selection of the } \\
\text { reported result }\end{array}$ \\
\hline Anic, $2016^{45}$ & Serious & Moderate & Low & Serious & Serious & Low & Moderate \\
\hline Boden, 201927 & Serious & Moderate & Low & Serious & Serious & Low & Moderate \\
\hline Deschasaux, $2018^{4}$ & Serious & Moderate & Low & Serious & Moderate & Low & Moderate \\
\hline Hodge, $2016^{46}$ & Serious & Serious & Low & Serious & Moderate & Low & Moderate \\
\hline Kane-Diallo, $2018^{14}$ & Serious & Moderate & Low & Serious & Moderate & Low & Moderate \\
\hline Maisonneuve, $2016^{47}$ & Critical & Moderate & Low & Serious & Serious & Low & Moderate \\
\hline Schulpen, $2018^{48}$ & Serious & Serious & Low & Serious & Moderate & Low & Serious \\
\hline Voortman, $2017^{24}$ & Serious & Moderate & Low & Serious & Moderate & Low & Moderate \\
\hline
\end{tabular}

Ixxvi Possible ratings of low, moderate, serious, critical, or no information determined using the "Risk of Bias for Nutrition Observational Studies" tool (RoBNObs) (Dietary Guidelines Advisory Committee. 2020. Scientific Report of the 2020 Dietary Guidelines Advisory Committee: Advisory Report to the Secretary of Agriculture and the Secretary of Health and Human Services. U.S. Department of Agriculture, Agricultural Research Service, Washington, DC.) 
Table 12. Description of studies that examined the relationship between dietary patterns and prostate cancerlxxvii

\begin{tabular}{llll}
\hline Study and & Intervention/Exposure and & Results & Confounding, Study Limitations, and \\
Participant & Outcomes & & Summary of Findings \\
Characteristics & &
\end{tabular}

Deschasaux, $2018^{4}$

PCS (European

Prospective

Investigation into

Cancer and Nutrition

(EPIC))

Denmark, France,

Germany, Greece,

Italy, the

Netherlands,

Norway, Spain,

Sweden, UK

Analytic N: 140729

Participants were

$100 \%$ male, $\sim 51$ yo,

$\sim 25.4 \mathrm{~kg} / \mathrm{m}^{2} \mathrm{BMI}, 43 \%$

never-smokers, $\sim 5.3$

$\mathrm{g} / \mathrm{d}$ alcohol, $\sim 20.9 \%$

inactive; $17.9 \%$ active

\section{Dietary patterns:}

Nutrient Profiling System of the British

Food Standards Agency dietary index (modified version) (FSAm-NPS DI) score, categorical (quintiles) and continuous (per 2 pt increment) [ - 15 (most healthy) to +40 (least healthy)]

- Overall diet score assigned based on energy, sugar, saturated fatty acid, sodium, fibres, proteins, and fruits/vegetables/legumes/nuts.

- Higher FSAm-NPS score had higher intakes of alcohol, energy and red and processed meat, lower intakes of dietary fibers, vegetables, fruit, fish, and lean meat.

Dietary assessment methods: FFQs or diet history questionnaire and 7-day dietary records, validated, at baseline, age $\sim 51 \mathrm{y}$

Outcome assessment methods:

Record linkage with population-based

\section{Significant:}

FSAm-NPS DI score at $51 \mathrm{y}$ and risk of prostate cancer after 15.3y f/u:

- Q1, $n=1192: H R: 1.00$

- Q2, $\mathrm{n}=1162: \mathrm{HR}: 0.99,95 \% \mathrm{Cl}: 0.91,1.07$

- Q3, $n=1365:$ HR: $1.05,95 \% \mathrm{Cl}: 0.97,1.15$

- Q4, $n=1471$ : HR: $1.06,95 \%$ Cl: 0.97, 1.16

- Q5, $n=1555:$ HR: $1.07,95 \%$ Cl: 0.98, 1.17

- $p$-trend $=0.04$

Continuous, per 2pt increment, $n=6745$ : HR: 1.03 , $95 \% \mathrm{Cl}: 1.00,1.06$; $p$-trend $=0.04$

\section{Non-Significant: N/A}

\section{Key confounders accounted for:}

Sex, age, education, alcohol intake, physical activity, smoking, BMI, height

\section{Other:}

Center, energy intake

\section{Limitations:}

- Did not account for race/ethnicity

- Did not account for dietary exposure earlier in life, that occurred prior to start of the study

- Only assessed dietary intake once at baseline; did not account for possible changes in dietary intake over $\mathrm{f} / \mathrm{u}$

- Did not account for missing data

- No preregistered statistical plan; potentia for selective outcome reporting

Funding Sources: French National Cancer Institute, European Commission, the International Agency for Research on Cancer

Ixxvii Abbreviations: AHEI-2010, Alternative Healthy Eating Index-2010; aMED, Alternative Mediterranean Diet Score ; BMI, body mass index; d, day; DP, Dietary pattern; \%E, \% of energy; EPIC, European Prospective Investigation into Cancer and Nutrition; FFQ, food frequency questionnaire; FSAm-NPS, Nutrient Profiling System of the British Food Standards Agency (modified version); FSAm-NPS DI, Nutrient Profiling System of the British Food Standards Agency dietary index; f/u, follow-up; HR, hazard ratio; MEDI-LITE, Mediterranean diet score; MDS, Mediterranean Diet Score; mMED, modified Mediterranean diet scores; mMEDr, modified Mediterranean diet scores without alcohol; mo, month(s); N/A, Not applicable; NIH, National Institutes of Health; NOVA, Ultra-processed food score; NS, Not significant; NR, Not reported; PCS, prospective cohort study; PNNS-GS, French National Nutrition Health Program-Guideline Score; pt, point; RCT, randomized controlled trial; WCRF/AICR, World Cancer Research Fund/American Institute for Cancer Research; wk, week(s); y, year(s) 


\section{Study and}

Participant

Characteristics

Excluded those with prevalent cancer;

cancer diagnosis in

first $2 y$ of f/u; missing

data; implausible

energy intake $(\sim 10 \%$

of original sample)

\section{Donnenfeld, $2015^{6}$}

PCS using data from an RCT

(SUpplémentation en

VItamines et

Minéraux

AntioXydants cohort)

France

Analytic N: 2753

Participants were $100 \%$ male, $\sim 49 \mathrm{yo}$, $34 \% \mathrm{BMI}>25 \mathrm{~kg} / \mathrm{m}^{2}$,

$48 \%$ never-smokers,

$\sim 18.8 \mathrm{~g} / \mathrm{d}$ alcohol,

$\sim 45 \% \geq 1 \mathrm{~h} / \mathrm{d}$ of

walking, $\sim 35 \%$ family

history of cancer

Excluded those with

prevalent cancer:

cancer diagnosis in

first 3y of f/u; $<6$ 24-hr

recalls within the first

$2 y$ of $f / u$; implausible
Intervention/Exposure and

\section{Outcomes}

Results

cancer registries, health insurance

records, pathology registries, and f/u

with study participants

\section{Dietary patterns:}

Nutrient Profiling System of the British

Food Standards Agency (modified

version) (FSAm-NPS) score,

categorical (quintiles) and continuous

(per 2 pt increment) [ -15 (most

healthy) to +40 (least healthy)]

- Overall diet score assigned based on energy, sugar, saturated fatty acid, sodium, fiber, proteins, and fruits/vegetables/legumes/nuts.

Dietary assessment methods: $6,24-$ hour dietary records during the first $2 y$ of f/up, age $\sim 49 y$, and every 2 mo, for first $2 y$ of $f / u$

\section{Outcome assessment methods:}

Participant report, medical record review, pathological reports

Confounding, Study Limitations, and Summary of Findings

Summary: Consuming a diet that scores higher (i.e., lower nutritional quality) on the Nutrient Profiling System of the British Food Standards Agency dietary index (modified version) (FSAmNPS DI) at 51 y was associated with decreased risk of prostate cancer after 15.3y f/u.

\section{Significant: N/A}

\section{Non-Significant:}

FSAm-NPS score at $49 \mathrm{y}$ and risk of prostate cancer after $12.6 y \mathrm{f} / \mathrm{u}$ :

- $\mathrm{Q} 1, \mathrm{n}=29: 1$

- Q2, n= 18: HR: 0.82, 95\% Cl: 0.45, 1.50

- Q3, $n=26: H R: 1.24,95 \%$ Cl: $0.71,2.14$

- $Q 4, n=16: H R: 0.83,95 \%$ Cl: $0.44,1.56$

- $Q 5, n=23: H R: 1.31,95 \%$ Cl: $0.74,2.33$

- $p$-trend $=0.4$

Continuous, $n=112$ : HR: $1.07,95 \% \mathrm{Cl}: 0.93,1.22$ p-trend $=0.3$

\section{Key confounders accounted for:}

Sex, age, education, alcohol intake, physical activity, smoking, BMI, height, family history of prostate cancer

Other:

Intervention group of the initial SU.VI.MAX trial, number of dietary records, baseline PSA

\section{Limitations:}

- Did not account for race/ethnicity

- Exposure occurred prior to start of $\mathrm{f} / \mathrm{u}$

- Only assessed dietary intake during first 2y of $\mathrm{f} / \mathrm{u}$; did not account for possible changes in dietary intake over $\mathrm{f} / \mathrm{u}$

- Did not account for missing data

- No preregistered statistical plan; potential for selective outcome reporting

Funding Sources: French Ministry of Health (DGS), National Institute for Prevention and Health Education (INPES)

Summary: Nutrient Profiling System of the British Food Standards Agency (modified version) (FSAm-NPS) score at $49 y$ was not 


\section{Study and}

Participant

Characteristics

Intervention/Exposure and

\section{Outcomes}

\section{energy intake ( $51 \%$}

of original sample)

\section{Fiolet, 2018}

\section{PCS (NutriNet-Santé)}

France

Analytic N: 22821

Participants were $100 \%$ male, $42.8 y o$, $23.8 \mathrm{~kg} / \mathrm{m} 2 \mathrm{BMI}, 83 \%$ never or former smokers, $\sim 7.8 \mathrm{~g} / \mathrm{d}$ alcohol, family history of cancer $34 \%$, moderate or high physical activity $\sim 65 \%$

Excluded those with prevalent cancer; $<18$ yo at baseline; <2 valid $24-\mathrm{hr}$ dietary records during first $2 \mathrm{y}$ $\mathrm{f} / \mathrm{u}$; diagnosis in first $2 y$ of $\mathrm{f} / \mathrm{u}(\sim 11 \%$ of original sample)

\section{Dietary patterns:}

- Ultra-processed food score (NOVA), categorical (quartiles)

- Main food groups contributing to NOVA score were sugary drinks, drinks, starchy foods and breakfast cereals, ultraprocessed fruits and vegetables, dairy products, meats, fish, and eggs processed meats, fats, and salty snacks

Dietary assessment methods: $3,24-$ hour dietary records every 6 months, assessed during the first $2 y$ of $f / u$, at age $\sim 49 y$

\section{Outcome assessment methods:}

Participant report, medical record review, pathological reports, linked to health insurance and mortality databases

Results

Confounding, Study Limitations, and Summary of Findings

significantly associated with risk of prostate

\section{Significant: N/A}

\section{Non-Significant:}

Ultra-processed food score at $49 y$ and prostate cancer after $5.4 \mathrm{y}$ f/u:

- Q1, n=96: HR: 1.00

- Q2, n=96: HR: 1.18, 95\% Cl: 0.89, 1.57

- Q3, n=59: HR: 0.95, 95\% Cl: 0.68, 1.32

- Q4, n=30: HR: 0.93, 95\% Cl: 0.61, 1.40

- $\quad p$-trend=0.6

Ultra-processed food score, continuous, and prostate cancer $(n=281)$ : HR: $0.98,95 \% \mathrm{Cl}: 0.83$, $1.16 ; p$-trend $=0.8$ cancer after 12.6 y $\mathrm{f} / \mathrm{u}$.

\section{Key confounders accou}

Sex, age, education, alcohol intake, physical activity, smoking, BMI, height, family history of the cancer

\section{Other:}

Energy intake without alcohol, number of 24 hour dietary records, intakes of lipids, sodium, and carbohydrates and western dietary pattern

\section{Limitations:}

- Did not account for race/ethnicity

- Exposure occurred prior to start of $\mathrm{f} / \mathrm{u}$

- $\quad$ Only assessed dietary intake during first $2 y$ of $\mathrm{f} / \mathrm{u}$; did not account for possible changes in dietary intake over $\mathrm{f} / \mathrm{u}$

- Did not account for missing data

- No preregistered statistical plan; potentia for selective outcome reporting

Funding Sources: Région lle-de-France, Cancéropôle lle-de-France, Ministère de la Santé, Institut de Veille Sanitaire, Institut

National de la Prévention etde l'Education pour la Santé, Région Ile-de-France, Institut Nationalde la Santé et de la Recherche Médicale, Institut National de la Recherche Agronomique (INRA), onservatoire National des Arts et Métiers and Université Paris 13 


\section{Study and}

Participant

Characteristics

Intervention/Exposure and

Outcomes
Results
Confounding, Study Limitations, and Summary of Findings

Summary: Ultra-processed food score at 49y was not significantly associated with risk of prostate cancer after $5.4 \mathrm{y} / \mathrm{f}$.

\section{Kane-Diallo, $2018^{14}$}

\section{PCS (NutriNet-Sante} study)

\section{France}

Analytic N: 11615

Participants were $100 \%$ male, $\sim 56.9 y$ $(\geq 45 \mathrm{y}), \sim 25 \mathrm{~kg} / \mathrm{m} 2$, $\sim 44 \%$ never-smokers, $9.7 \mathrm{~g} / \mathrm{d}$ alcohol, $\sim 80 \%$ high or moderate physical activity, $51 \%$ family history of

cancer

Excluded those with prevalent cancer; $<3$ 24-hr recalls within the first year of $f / u$; missing f/u data;

implausible energy intake; $<45 y$ y $(21 \%$ of original sample)

\section{Dietary patterns:}

- "Pro plant-based" dietary score, categorical (tertiles)

- Higher in plant foods: vegetables, legumes, fruits cereal products, potatoes, nuts, vegetables oils

- Lower in animal foods: red and processed meat, eggs, animal fat, dairy products, seafood

Dietary assessment methods: 3, 24hour dietary records every 6 months, assessed during the first $2 \mathrm{y}$ of $\mathrm{f} / \mathrm{u}$, at age $\sim 57 y$

\section{Outcome assessment methods:}

Participant report, medical record review, pathological reports, linked to health insurance and mortality databases

\section{Significant: N/A}

\section{Non-Significant:}

"Pro plant-based" dietary score at $49 y$ and risk of prostate cancer after $4.3 \mathrm{y} / \mathrm{u}$ :

- T1, $n=84 / 3849:$ HR: 1.00

- T2, n=85/3679: HR: 0.90, 95\% Cl: 0.66, 1.23

- T3, n=74/3844: HR: 0.76, 95\% Cl: 0.55, 1.06

- $\quad$-trend $=0.1$

\section{Key confounders accounted for:}

Sex, age, education, alcohol intake (in adults), physical activity, smoking, height, BMI, family history of the cancer outcome

Other:

Energy intake without alcohol, number of 24-hr dietary records, lipids intake

\section{Limitations:}

- Did not account for race/ethnicity

- Exposure occurred prior to start of $f / u$

- Only assessed dietary intake during first 2y of $\mathrm{f} / \mathrm{u}$; did not account for possible changes in dietary intake over $\mathrm{f} / \mathrm{u}$

- Did not account for missing data

- No preregistered statistical plan; potential for selective outcome reporting

Funding Sources: Ministere de la Sante, Institut de Veille Sanitaire, Institut National de la Prevention et de l'Education pour la Sante Region lle-de-France, Institut National de la Sant e et de la Recherche Medicale, Institut National de la Recherche Agronomique,

Conservatoire National des Arts et Metiers, The French National Cancer Institute

Summary: "Pro plant-based" dietary score at $57 y$ was not significantly associated with risk of prostate cancer after $4.3 y$ f/u. 


\section{Study and \\ Participant \\ Characteristics \\ Lavalette, $2018^{16}$ \\ PCS (NutriNet-Sante study)}

\section{France}

Analytic N: 11018

Participants were $100 \%$ male, $\sim 54$ yo (all $>40 \mathrm{y}$, $\sim 24.5 \mathrm{~kg} / \mathrm{m} 2$, $\sim 44 \%$ never-smokers, $\sim 9.4 \mathrm{~g} / \mathrm{d}$ alcohol, $78.2 \%$ high or moderate physical activity, $\sim 49 \%$ family history of cancer

Excluded those with prevalent cancer; $<3$ 24-hr recalls within the first year of $f / u$; missing f/u data; implausible energy intake; $<40 y(>50 \%$ of original sample)

\section{Intervention/Exposure and}

\section{Outcomes}

\section{Dietary patterns:}

- Alternate Healthy Eating Index 2010 (AHEI-2010)|xxviii, categorical (quintiles) and continuous (per 2 pt increment)

- Positive components:

Vegetables (not potatoes,

French fries), Fruit, Whole

Grains, Long-Chain Fats (EPA + DHA), PUFA

- Neutral components: Alcohol

- Negative components: Red and Processed Meat, Sugar Sweetened Beverages and Fruit Juice, Trans FA, Sodium

- Mediterranean diet score (MEDILITE)|'xxix , categorical (quintiles) and continuous (per $2 \mathrm{pt}$ increment)

- Positive components: Vegetables, legumes, fruit and nuts, cereals, fish, olive oil

- Neutral components: Alcohol

- Negative components: Meat, dairy products

- French National Nutrition Health Program-Guideline Score (PNNSGS) ${ }^{1 \times x x}$, categorical (quintiles) and continuous (per 2 pt increment)

\section{Results}

\section{Significant: N/A}

\section{Non-Significant:}

AHEI-2010 score at $~ 55 y$ and risk of prostate cancer after $8.5 \mathrm{y} / \mathrm{u}$ :

- Q1, $n=32 / 2171: H R: 1.00$

- Q2, $n=42 / 2162: H R: 0.93,95 \% \mathrm{Cl}: 0.59$, 1.48

- Q3, $n=55 / 2149: H R: 1.14,95 \% \mathrm{Cl}: 0.73$, 1.77

- Q4, n=48/2156: HR: 0.90, 95\% Cl: 0.57, 1.42

- Q5, $n=45 / 2158: H R: 0.82,95 \% \mathrm{Cl}: 0.51$, 1.31

- $p$-trend $=0.3$

AHEI-2010 score, continuous, $n=222 / 10796$ : HR: $0.96,95 \% \mathrm{Cl}: 0.85,1.08$; $\mathrm{p}$-trend $=0.5$

MEDI-LITE score at $\sim 49 y$ and risk of prostate cancer after $8.5 \mathrm{y} / \mathrm{u}$ :

- Q1, $n=38 / 2473: H R: 1.00$

- $Q 2, n=32 / 1530: H R: 1.16,95 \% \mathrm{Cl}: 0.72$, 1.86

- Q3, $n=37 / 1711: \mathrm{HR}: 1.12,95 \% \mathrm{Cl}: 0.71$ 1.76

- Q4, n=74/3043: HR: $1.14,95 \%$ Cl: 0.77 , 1.70
Confounding, Study Limitations, and Summary of Findings

(n)

Ixxviii Chiuve SE, Fung TT, Rimm EB, Hu FB, McCullough ML, Wang M, et al. Alternative dietary indices both strongly predict risk of chronic disease. J Nutr 2012;142:1009-18.

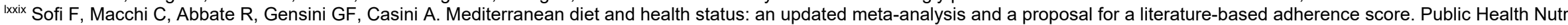
2014;17: 2769-82.

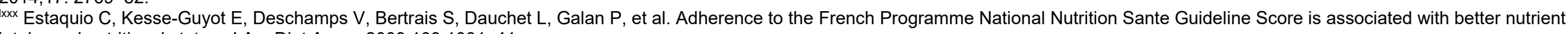
intake and nutritional status. J Am Diet Assoc 2009;109:1031-41.

Sex, age, education, alcohol intake, physical activity, smoking, height, BMI, family history of the cancer

\section{Other:}

Number of 24-hours dietary records, energy intake without alcohol

\section{Limitations:}

- Did not account for race/ethnicity

- Exposure occurred prior to start of f/u

- Only assessed dietary intake during first $2 y$ of $f / u$; did not account for possible changes in dietary intake over $\mathrm{f} / \mathrm{u}$

- Did not account for missing data

- No preregistered statistical plan; potentia for selective outcome reporting

Funding Sources: Ministere de la Sante, Institut de Veille Sanitaire, Institut National de la

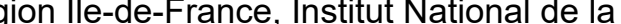

Sant_e et de la Recherche Medicale, Institut

National de la Recherche Agronomique,

Conservatoire National des Arts et Metiers, The French National Cancer Institute 


\section{Study and}

Participant

Characteristics
Intervention/Exposure and

\section{Outcomes}

- Positive components: Vegetables and Fruit,

Seafood, Vegetable Fat

- Neutral components: Breads, cereals, potatoes, legumes, meat and poultry, seafood, and eggs, milk and dairy products, alcohol

- Negative components: Sweetened foods, soda, added fat, salt

Dietary assessment methods: 3, 24hour dietary records every $6 \mathrm{mo}$, assessed during the first $2 y$ of $f / u$, at age $\sim 55 y$

\section{Outcome assessment methods:}

Participant report, medical record review, pathological reports, linked to health insurance and mortality databases

Schulpen, 201951
Nested Case-Control
Study (Netherlands
Cohort Study
(NLCS))

\section{Dietary patterns:}

- alternate Mediterranean diet scores (aMED) $)^{\mathrm{Ixx}}$, categorical (tertiles) and continuous (per 2 pt increment), and without alcohol (aMEDr)c

- Positive components: Vegetables (not potatoes),
Results

- Q5, n=41/2039: HR: 0.95, 95\% Cl: 0.61, 1.50

- $\quad$-trend $=0.9$

MEDI-LITE score, continuous, $n=222 / 10796$ : HR: $0.98,95 \% \mathrm{Cl}: 0.93,1.04 ; p$-trend $=0.5$

PNNS-GS score at $\sim 49 y$ and risk of prostate cancer after $8.5 \mathrm{y} f / \mathrm{u}$ :

- $Q 1, n=35 / 2161: H R: 1.00$

- Q2, $\mathrm{n}=38 / 1998$ : HR: $0.88,95 \% \mathrm{Cl}$ : 0.55 , 1.40

- Q3, $n=46 / 2292: H R: 0.85,95 \%$ Cl: 0.55 , 1.34

- Q4, n=44/2086: HR: $0.74,95 \% \mathrm{Cl}: 0.47$, 1.17

- Q5, n=59/2259: HR: 0.83, 95\% Cl: 0.54, 1.28

- $\quad \mathrm{p}$-trend $=0.3$

PNNS-GS score, continuous, $n=222 / 10796$ : $H R$ : $0.97,95 \% \mathrm{Cl}: 0.89,1.05 ; \mathrm{p}$-trend $=0.4$

\section{Significant:}

\section{aMEDr:}

aMEDr score at $61 \mathrm{y}$ and risk of nonadvanced prostate cancer after $20.3 \mathrm{y} / \mathrm{u}$ :

- $0-3, n=854:$ HR: 1.00

- 4-5, n=1,048: HR: 1.14, 95\% Cl: 0.99, 1.31

- $6-8, n=427:$ HR: $1.22,95 \%$ Cl: $1.01,1.48$
Confounding, Study Limitations, and Summary of Findings

Summary: AHEI-2010, MEDI-LITE, and PNNSGS scores at 55y were not significantly associated with risk of prostate cancer after $8.5 y$ f/u.

\section{Key confounders accounted for:}

Sex, age, race/ethnicity, education, alcohol intake, physical activity, smoking, BMI, family history of the cancer outcome

\section{Other:}

Daily energy intake

Limitations:

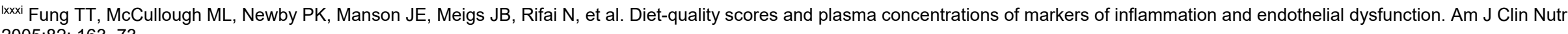
2005;82: 163-73. 


\section{Study and}

Participant

Characteristics

Intervention/Exposure and

\section{Outcomes}

\section{The Netherlands}

Analytic N: 5748

Participants were $100 \%$ male, $\sim 61$ yo (55-69y), $\sim 24.9 \mathrm{~kg} / \mathrm{m}^{2}$ $\mathrm{BMI}, \sim 68 \%$ nonsmokers; $\sim 8.5 \mathrm{~g} / \mathrm{d}$ alcohol, $61.8 \mathrm{~min} / \mathrm{d}$ non-occupational physical activity, 3.1\% family history of prostate cancer

Excluded those with prevalent cancer (except skin); missing data on diet and alcohol ( $8 \%$ of original sample) legumes, fruits, nuts, whole grains, fish, MUFA/SFAs

- Neutral components: Alcohol

- Negative components: Red and processed meat modified Mediterranean diet scores (mMED) ) $^{\mid x \times x i i}$, categorical (tertiles) and continuous (per $2 \mathrm{pt}$ increment), and without alcohol

(mMEDr)

- Positive components:

Vegetables, legumes, fruit, cereals, fish,

\section{MUFA+PUFA/SFA}

- Neutral components: Alcohol

- Negative components: Meat, dairy products

- WCRF/AICR score, ${ }^{1 \times x x i i i}$ diet only, continuous (per SD increment), and without alcohol

- Positive components:

Vegetables and fruit, dietary fiber

- Negative components: Red and processed meat, sugary drinks, alcohol, sodium, energy-dense foods

Dietary assessment methods: 150 item, validated FFQ at baseline, age $\sim 61 \mathrm{y}$

\section{Results}

- $\quad$-trend=0.037

aMEDr score and nonadvanced prostate cancer, continuous, per 2 pts, n=2,329: HR: 1.12, 95\% Cl: $1.04,1.22$

aMEDr score at 61y per SD increment and nonadvanced prostate cancer after $20.3 \mathrm{y} / \mathrm{u}$ : HR: $1.09,95 \% \mathrm{Cl}: 1.03,1.17$

aMEDr score and prostate cancer, continuous, per 2 pts, n=3,868: HR: $1.09,95 \% \mathrm{Cl}: 1.01,1.17$ aMEDr score at 61y per SD increment and risk of prostate cancer after $20.3 \mathrm{y}$ f/u: HR: $1.07,95 \% \mathrm{Cl}$ : $1.01,1.13$

\section{aMED:}

aMED score at 61y and risk of nonadvanced prostate cancer after $20.3 \mathrm{y} / \mathrm{u}$ :

- $0-3, n=666:$ HR: 1.00

- 4-5, n=1,008: HR: $1.07,95 \% \mathrm{Cl}: 0.92$, 1.25

- 6-9, n=655: HR: $1.28,95 \% \mathrm{Cl}: 1.08,1.53$

- $p$-trend $=0.012$

aMED score, continuous, per 2 pts, and nonadvanced prostate cancer, $\mathrm{n}=2,329$ : HR: 1.12 , 95\% Cl: $1.04,1.21$

aMED score at 61y per SD increment and prostate cancer after $20.3 y$ f/u: HR: $1.07,95 \% \mathrm{Cl}$ : $1.01,1.14$
Confounding, Study Limitations, and Summary of Findings

- $\quad$ Did not account for race/ethnicity, physical activity, smoking

- Exposure occurred prior to start of $f / u$

- Only assessed dietary intake once at baseline; did not account for possible changes in dietary intake over f/u

- Did not account for missing data

- $\quad$ No preregistered statistical plan; serious potential for selective outcome reporting

Funding Sources: Wereld Kanker Onderzoek Fonds Nederland, World Cancer Research

Fund International

\section{Summary:}

Higher aMED score with and without alcohol was associated with significantly increased risk of nonadvanced prostate cancer after 20.3y f/u. However, aMED scores, with and without alcohol, were not significantly associated with total risk of prostate cancer or risk of advanced prostate cancer after $20.3 y \mathrm{f} / \mathrm{u}$.

mMED score, with and without alcohol, was not significantly associated with risk of prostate cancer, including nonadvanced and advanced prostate cancer, after $20.3 y \mathrm{f} / \mathrm{u}$.

WCRF/AICR diet only score, with and without alcohol, was not significantly associated with

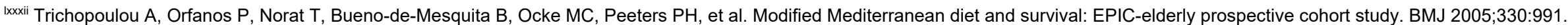

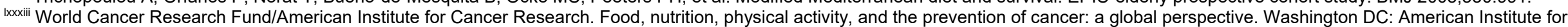
Cancer Research; 2007. 


\section{Study and} Participant Characteristics
Intervention/Exposure and Outcomes

\section{Outcome assessment methods:}

Netherlands Cancer Registry and the nationwide

Dutch Pathology Registry, and review of pathology records
Results

aMED score, continuous, per 2 pts, and

nonadvanced prostate cancer, $n=3,868$ : HR: 1.09 , 95\% Cl: $1.01,1.17$

aMED score at 61y per SD increment and

nonadvanced prostate cancer after 20.3y f/u: HR:

$1.10,95 \% \mathrm{Cl}: 1.03,1.17$

\section{mMEDr:}

mMEDr, continuous, per 2 pts, $n=2,329: H R: 1.11$, $95 \% \mathrm{Cl}: 1.02,1.21$

\section{MMED:}

mMED, continuous, per 2 pts, $n=3,868$ : HR: 1.07 , 95\% Cl: $1.00,1.16$

mMED score at $61 \mathrm{y}$ and risk of nonadvanced prostate cancer after $20.3 \mathrm{y} / \mathrm{u}$ :

- $\quad 0-3, n=571:$ HR: 1.00

- 4-5, $n=1,069$ : HR: $0.99,95 \% \mathrm{Cl}: 0.84$

$$
1.16
$$

- 6-9, n=689: HR: 1.18, 95\% Cl: 0.99, 1.41

- $p$-trend $=0.034$

mMED, continuous, per 2 pts, $n=2,329$ : HR: 1.11 , $95 \% \mathrm{Cl}: 1.02,1.20$

\section{Non-Significant:}

\section{aMEDr:}

aMEDr score at $61 \mathrm{y}$ and risk of prostate cancer after 20.3y f/u:
Confounding, Study Limitations, and Summary of Findings risk of prostate cancer, including nonadvanced and advanced prostate cancer, after 20.3y f/u.

\footnotetext{
- $0-3, n=1,473:$ HR: 1.00
} 


\section{Study and}

Participant

Characteristics
Intervention/Exposure and

Outcomes
Results

Confounding, Study Limitations, and Summary of Findings

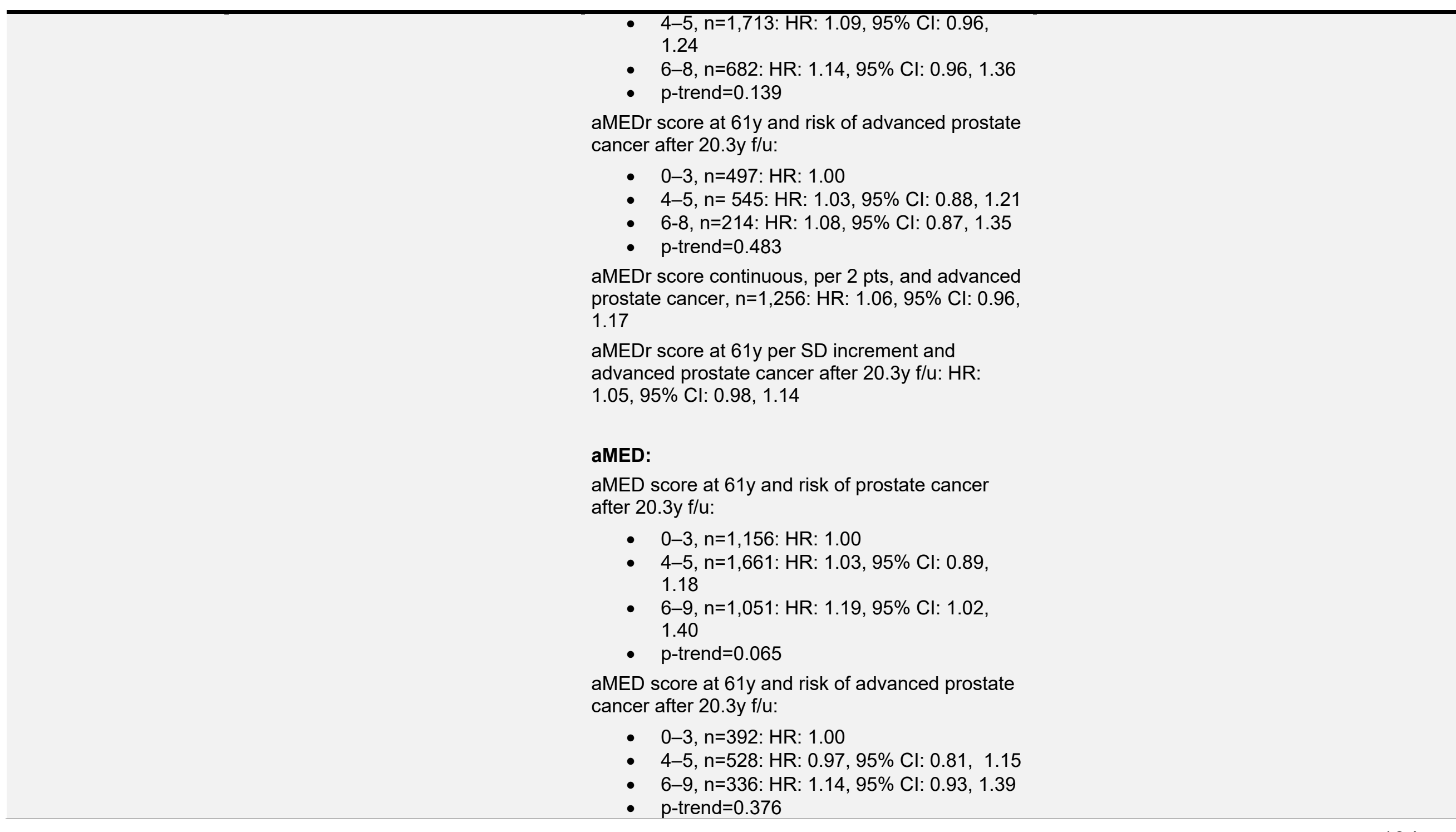




\section{Study and}

Participant

Characteristics
Intervention/Exposure and

Outcomes
Results

aMED score, continuous, per 2 pts, and advanced prostate cancer $n=1,256$ : HR: $1.07,95 \% \mathrm{Cl}: 0.98$, 1.17

aMED score at 61y per SD increment and advanced prostate cancer after 20.3y f/u: HR: $1.07,95 \% \mathrm{Cl}: 0.99,1.15$

\section{mMEDr:}

mMEDr score at 61y and risk of prostate cancer after 20.3y f/u:

- $\quad 0-3, n=1,329:$ HR: 1.00

- 4-5, n=1,857: HR: 1.10, 95\% Cl: 0.97 , 1.26

- 6-8, n= 682: HR: $1.10,95 \% \mathrm{Cl}: 0.92,1.31$

- $p$-trend $=0.285$

mMEDr, continuous, per 2 pts, $n=3,868$ : HR: 1.07 , 95\% Cl: 0.99, 1.16

mMEDr score at $61 \mathrm{y}$ and risk of nonadvanced prostate cancer after $20.3 \mathrm{y} / \mathrm{u}$ :

- $\quad 0-3, n=790:$ HR: 1.00

- 4-5, $n=1,111$ : HR: $1.11,95 \% \mathrm{Cl}: 0.96$, 1.28

- 6-8, n= 428: HR: $1.17,95 \%$ Cl: 0.97, 1.41

- $p$-trend $=0.111$

mMEDr score at 61y and risk of advanced prostate cancer after $20.3 \mathrm{y} / \mathrm{u}$ :

- $0-3, n=437:$ HR: 1.00

- 4-5, $n=614:$ HR: $1.12,95 \% \mathrm{Cl}: 0.95,1.32$

- 6-8, n=205: HR: 1.01, 95\% Cl: 0.81, 1.26

- $\mathrm{p}$-trend $=0.889$
Confounding, Study Limitations, and Summary of Findings 


\section{Study and}

Participant

Characteristics
Intervention/Exposure and

Outcomes
Results

Confounding, Study Limitations, and Summary of Findings

mMEDr, continuous, per 2 pts, $n=1,256$ : HR: 1.02, 95\% Cl: 0.92, 1.13

\section{MMED:}

$\mathrm{mMED}$ score at $61 \mathrm{y}$ and risk of prostate cancer after 20.3y f/u:

- $\quad 0-3, n=955:$ HR: 1.00

- 4-5, $n=1,817$ : HR: $1.00,95 \% \mathrm{Cl}: 0.87$, 1.16

- 6-9, n=1,096: HR: $1.12,95 \% \mathrm{Cl}: 0.95$, 1.32

- $p$-trend $=0.122$

mMED score at $61 \mathrm{y}$ and risk of advanced prostate cancer after 20.3y f/u:

- $\quad 0-3, n=310:$ HR: 1.00

- 4-5, n=612: HR: $1.05,95 \% \mathrm{Cl}: 0.87,1.26$

- 6-9, n=334: HR: 1.07, 95\% Cl: 0.87, 1.31

- $p$-trend $=0.577$

mMED, continuous, per 2 pts, $n=1,256$ : HR: 1.04 , 95\% Cl: 0.95, 1.14

\section{WCRF/AICR, diet only:}

WCRF/AICR score at $61 \mathrm{y}$, excluding alcohol, per $\mathrm{SD}$ increment and risk of prostate cancer after 20.3y f/u: HR: 1.03, 95\% Cl: 0.97, 1.09

WCRF/AICR score at $61 \mathrm{y}$, excluding alcohol, per SD increment and risk of nonadvanced prostate cancer after $20.3 \mathrm{y}$ f/u: HR: $1.03,95 \% \mathrm{Cl}: 0.97$, 1.10

WCRF/AICR score at 61y, excluding alcohol, per SD increment and risk of advanced prostate 


\section{Study and}

Participant

Characteristics
Intervention/Exposure and

Outcomes
Results
Confounding, Study Limitations, and Summary of Findings

cancer after 20.3y f/u: HR: 1.05, 95\% Cl: 0.97,

1.13

WCRF/AICR score, including alcohol, at $61 \mathrm{y}$ per SD increment and risk of prostate cancer after 20.3y f/u: HR: 1.04, 95\% Cl: 0.97, 1.10

WCRF/AICR score, including alcohol, at 61y per SD increment and risk of nonadvanced prostate cancer after $20.3 y$ f/u: HR: $1.04,95 \%$ Cl: 0.98 ,

1.11

WCRF/AICR score, including alcohol, at 61y per SD increment and risk of advanced prostate cancer after $20.3 y$ f/u: HR: $1.04,95 \%$ Cl: 0.97 , 1.13

\section{Shin, $2018^{37}$}

PCS (The Japan Public Health Centerbased Prospective Study (JPHC)) Japan

Analytic N: 43469

\section{Participants were} $100 \%$ male, $\sim 56$ yo (40-69y), 23.5kg/m2, $54 \%$ non-smokers, $\sim 66 \%$ regular drinkers

Excluded those with prevelant prostate cancer, implausible energy intake $(6 \%$ of study

\section{Dietary patterns:}

Adherence to 3 dietary patterns were derived using exploratory factors analysis, categorical (quintiles):

- "Prudent" pattern: high loadings for vegetables, fruit, noodles, potatoes, soy products, mushrooms, and seaweed

- "Westernized" pattern: higher loadings for meat and processed meat, eel, dairy products, fruit juice, coffee, tea, soft drink, sauces, and alcohol

- "Traditional" pattern: higher loadings for pickles, seafood, fish (oily, salty, and lean fish, and salmon), chicken, and sake

Dietary assessment methods: 138item, validated, FFQ at baseline, age $\sim 56 \mathrm{y}$

\section{Significant:}

\section{“Westernized" Pattern:}

Adherence to the "westernized" pattern at 56y and risk of total prostate cancer after 13.8y f/u:

- Q1, n=274: HR: 1.00

- Q2, n=247: HR: $1.06,95 \% \mathrm{Cl}: 0.89,1.26$

- Q3, $n=219:$ HR: $1.04,95 \%$ Cl: 0.86, 1.24

- Q4, n=231: HR: $1.22,95 \% \mathrm{Cl}: 1.02,1.47$

- Q5, n=185: HR: 1.22, 95\% Cl: 1.00, 1.49

- $p$-trend $=0.021$

Adherence to the "westernized" pattern at 56y and risk of localized prostate cancer after $13.8 \mathrm{y} / \mathrm{u}$ :

- Q1, $n=184: H R: 1.00$

- Q2, $n=167: H R: 1.03,95 \%$ Cl: $0.83,1.27$

- Q3, $n=155:$ HR: $1.03,95 \%$ Cl: $0.83,1.29$

- Q4, $n=160: H R: 1.18,95 \%$ Cl: $0.95,1.47$

- Q5, $n=136:$ HR: $1.24,95 \%$ Cl: 0.97, 1.57

- $p$-trend $=0.045$

\section{Key confounders accounted for:}

Sex, age, race/ethnicity, marital status, alcohol intake, smoking, BMI

Other: Public health center area, vitamin supplement use

\section{Limitations:}

- Did not account for physical activity, family history of the cancer

- Exposure may be misclassified

- Exposure occurred prior to start of $f / u$

- Only assessed dietary intake once at baseline; did not account for possible changes in dietary intake over $\mathrm{f} / \mathrm{u}$

- Did not account for missing data

- No published protocol; potential for selective outcome reporting

Funding Sources: National Cancer Center 


\section{Study and}

Participant

Characteristics
Intervention/Exposure and

Outcomes
Results

\section{Non-Significant:}

Patient $f / u$, hospital records, population-based cancer registries, death certificates

\section{"Prudent" Pattern:}

Adherence to the "prudent" pattern at 56y and risk of total prostate cancer after $13.8 \mathrm{y} / \mathrm{u}$ :

- Q1, $n=193:$ HR: 1.00

- Q2, $n=185:$ HR: $0.91,95 \%$ Cl: 0.74, 1.12

- Q3, $n=242:$ HR: $1.05,95 \% \mathrm{Cl}: 0.87,1.27$

- $Q 4, n=255:$ HR: $1.01,95 \%$ Cl: $0.84,1.23$

- Q5, n=281: HR: 0.93, 95\% Cl: 0.76, 1.12

- $p$-trend $=0.715$

Adherence to the "prudent" pattern at 56y and risk of localized prostate cancer after 13.8y f/u:

- Q1, $n=123: H R: 1.00$

- Q2, $n=128: H R: 0.98,95 \% \mathrm{Cl}: 0.77,1.26$

- Q3, n=177: HR: $1.19,95 \% \mathrm{Cl}: 0.94,1.50$

- Q4, $n=184:$ HR: $1.15,95 \% \mathrm{Cl}: 0.91,1.45$

- Q5, n=190: HR: 1.00, 95\% Cl: 0.79, 1.27

- $p$-trend $=0.555$

Adherence to the "prudent" pattern at 56y and risk of advanced prostate cancer after $13.8 \mathrm{y} / \mathrm{u}$ :

- Q1, n=59: HR: 1.00

- Q2, $n=50: H R: 0.82,95 \%$ Cl: $0.56,1.21$

- Q3, $n=54:$ HR: $0.80,95 \%$ Cl: $0.55,1.16$

- Q4, n=57: HR: $0.76,95 \%$ Cl: 0.52, 1.10

- Q5, $n=73:$ HR: $0.78,95 \%$ Cl: 0.55, 1.12

- $p$-trend $=0.135$
Confounding, Study Limitations, and Summary of Findings

Research and Development Fund, Ministry of Health, Labour and Welfare of Japan, National Research Foundation of Korea

Summary: Higher adherence to a "westernized" pattern at 56y was associated with associated with significantly increased risk of total and localized prostate cancer after $13.8 \mathrm{y} f / \mathrm{u}$.

Adherence to the "westernized" pattern was not significantly associated with risk of advanced prostate cancer.

Adherence to the "prudent" pattern and the "traditional" pattern at 56y were not significantly associated with risk of advanced prostate cancer after 13.8y f/u. 


\section{Study and}

Participant

Characteristics
Intervention/Exposure and

Outcomes
Results

Confounding, Study Limitations, and Summary of Findings

Adherence to the "westernized" pattern at 56y and risk of advanced prostate cancer after 13.8y f/u:

- Q1, n=73: HR: 1.00

- Q2, $n=65: H R: 1.12,95 \% \mathrm{Cl}: 0.80,1.57$

- Q3, $n=56: H R: 1.09,95 \% \mathrm{Cl}: 0.76,1.56$

- Q4, $n=56: H R: 1.25,95 \%$ Cl: $0.87,1.81$

- Q5, n=43: HR: $1.23,95 \% \mathrm{Cl}: 0.82,1.84$

- $\quad$-trend $=0.233$

\section{“Traditional" Pattern:}

Adherence to the "traditional" pattern at 56y and risk of total prostate cancer after 13.8y f/u:

- Q1, $n=253:$ HR: 1.00

- Q2, n=230: HR: $1.17,95 \% \mathrm{Cl}: 0.95,1.45$

- Q3, $n=215:$ HR: $1.10,95 \% \mathrm{Cl}: 0.87,1.39$

- Q4, n=229: HR: $1.10,95 \% \mathrm{Cl}: 0.86,1.40$

- Q5, n=229: HR: $1.01,95 \% \mathrm{Cl}: 0.79,1.29$

- $\mathrm{p}$-trend $=0.895$

Adherence to the "traditional" pattern at 56y and risk of localized prostate cancer after $13.8 \mathrm{y}$ f/u:

- Q1, n=182: HR: 1.00

- Q2, $n=146: H R: 1.08,95 \% \mathrm{Cl}: 0.83,1.40$

- Q3, $n=147:$ HR: $1.06,95 \%$ Cl: $0.80,1.41$

- Q4, n=165: HR: 1.10, 95\% Cl: 0.82, 1.48

- Q5, n=160: HR: 0.97, 95\% Cl: 0.72, 1.31

- $p$-trend=0.869

Adherence to the "traditional" pattern at 56y and risk of advanced prostate cancer after $13.8 \mathrm{y} / \mathrm{u}$ :

- Q1, n=54: HR: 1.00

- Q2, n=65: HR: 1.36, 95\% Cl: 0.89, 2.07

- Q3, $n=55: H R: 1.16,95 \% \mathrm{Cl}: 0.73,1.84$ 


\section{Study and}

Participant

Characteristics
Intervention/Exposure and

Outcomes
Results
Confounding, Study Limitations, and Summary of Findings
- Q4, n=54: HR: 1.05, 95\% Cl: 0.65, 1.71

- Q5, n=65: HR: $1.16,95 \%$ Cl: 0.72, 1.88

- $p$-trend $=0.830$

\section{Tantamango-Bartley, Dietary patterns:}

$2016^{50}$

PCS (Adventist Health Study-2 (AHS2))

\section{United States}

Analytic N: 27188

Participants were $100 \%$ male, $\geq 30$ yo $(73 \%>59 \mathrm{y}), 66 \%$ $\mathrm{BMI}>25 \mathrm{~kg} / \mathrm{m}^{2}, 74 \%$ never smokers, $9 \%$ used alcohol in last $2 \mathrm{y}$ $\sim 15 \%$ family history of prostate cancer, $\sim 19 \%$ no vigorous exercise

\section{Excluded subjects} from ME and WI (no cancer registry), with prevalent cancers, self-reported cancer with no medical record verification, missing or invalid dietary data, age $<25 y$, missing
- "Vegans"|xxxiv: Red meat, poultry, fish; eggs; and dairy < 1 time/mo

- "Lacto-ovo-vegetarian": Red meat, poultry, and fish $<1$ time/mo, eggs or dairy $>1$ time/mo

- "Pesco-vegetarian": Red meat or poultry $<1$ time/mo, fish $>1$ time/mo, and eggs/dairy in any amount

- "Semi-vegetarian": Red meat or poultry $>1$ time/mo, and all meats combined (including fish) $<1$ time/wk and eggs/dairy in any amount

- "Nonvegetarians": Red meat and poultry $>1$ time/mo and all meats combined (including fish) $>1$ time/wk, and eggs/dairy in any amount

"Vegetarians" vs. "nonvegetarians" consumed higher amounts of fruits, vegetables, avocados, non-fried potatoes, whole grains, legumes, soy foods, nuts and seeds, and was observed among vegetarians; and lower amounts of meats, dairy products, eggs, refined grains, added

\section{Significant:}

Dietary pattern at $>30 \mathrm{y}$ and risk of overall prostate cancer after $7.8 \mathrm{y} / \mathrm{u}$ :

- "Vegan”, n=59: HR: 0.66, 95\% Cl: 0.50, 0.87

- "Lacto-ovo-vegetarian", n=333: HR: 0.96, 95\% $\mathrm{Cl}: 0.83,1.12$

- "Pesco-vegetarian", n=121: HR: 1.07, 95\% Cl: $0.88,1.31$

- "Semi-vegetarian", n=63: HR: 1.18, 95\% Cl: $0.91,1.54$

- "Nonvegetarian", n=503: HR: 1.00

When results were stratified by race, results were only significant in white subjects.

\section{Non-Significant:}

Dietary pattern at $>30 y$ and risk of advanced prostate cancer after $7.8 \mathrm{y} / \mathrm{u}$ :

- “Vegan”, n=15: HR: 0.78, 95\% Cl: 0.45, 1.35

- "Lacto-ovo-vegetarian", n=70: HR: 0.91 $95 \% \mathrm{Cl}: 0.66,1.24$

- “Pesco-vegetarian”, n=28: HR: 1.10, 95\% $\mathrm{Cl}: 0.72,1.68$
Key confounders accounted for:

Sex, age, race/ethnicity, education, alcohol intake, BMI, family history of the cancer

\section{Other:}

Energy intake, screening for prostate cancer

\section{Limitations:}

- Did not account for physical activity, smoking

- Exposure occurred prior to start of $f / u$

- Only assessed dietary intake once at baseline; did not account for possible changes in dietary intake over f/u

- Did not account for missing data

- No published protocol; potential for selective outcome reporting

Funding Sources: NIH, USDA, World Cancer Research Fund International

Summary: Consuming a "vegan" diet vs. a "nonvegetarian" diet was associated with a significantly lower risk of prostate cancer after $7.8 y \mathrm{f} / \mathrm{u}$. When stratified by race, results were only significant in white participants.

Ixxiv Orlich MJ, Jaceldo-Siegl K, Sabate J, et al. Patterns of food consumption among vegetarians and non-vegetarians. Br J Nutr. 2014; 112:1644-1653. [PubMed: 25247790] 


\section{Study and}

Participant

Characteristics

\section{Intervention/Exposure and}

Outcomes

fats, sweets, snack foods and non-

data on age/sex, implausible energy

intake $(\sim 20 \%$ of

original sample)
Results

- "Semi-vegetarian", n=13: HR: 1.09, 95\% Cl: $0.61,1.95$

- "Nonvegetarian", $n=111:$ HR: 1.00
Confounding, Study Limitations, and Summary of Findings

Consuming "vegan", "vegetarian", and "nonvegetarian" diets were not significantly associated with risk of advanced prostate cancer.

Dietary assessment methods: $>220$ -

item, validated FFQ, at baseline, $>30 y$

Outcome assessment methods:

State cancer registries, patient followup with medical record verification 
Table 13. Summary of the results from studies that examined the relationship between dietary patterns and prostate cancer ${ }^{\mid x x x v}$

\begin{tabular}{|c|c|c|c|}
\hline $\begin{array}{c}\text { Article, Study } \\
\text { Design, Country }\end{array}$ & Dietary Patterns & Significant Results & Non-Significant Results \\
\hline $\begin{array}{l}\text { Deschasaux, } \\
2018^{4} \\
\text { PCS (European } \\
\text { Prospective } \\
\text { Investigation into } \\
\text { Cancer and } \\
\text { Nutrition (EPIC)) } \\
\text { Denmark, France, } \\
\text { Germany, } \\
\text { Greece, Italy, the } \\
\text { Netherlands, } \\
\text { Norway, Spain, } \\
\text { Sweden, UK }\end{array}$ & $\begin{array}{l}\text { Nutrient Profiling System of the } \\
\text { British Food Standards Agency } \\
\text { dietary index (modified version) } \\
\text { (FSAm-NPS DI) } \\
\text { - Overall score based on } \\
\text { energy, sugar, saturated fatty } \\
\text { acid, sodium, fibres, proteins, } \\
\text { and } \\
\text { fruits/vegetables/legumes/nuts. } \\
\text { Higher FSAm-NPS DI score } \\
\text { had higher intakes of alcohol, } \\
\text { energy and red and } \\
\text { processed meat, lower } \\
\text { intakes of dietary fibres, } \\
\text { vegetables, fruit, fish, and } \\
\text { lean meat }\end{array}$ & 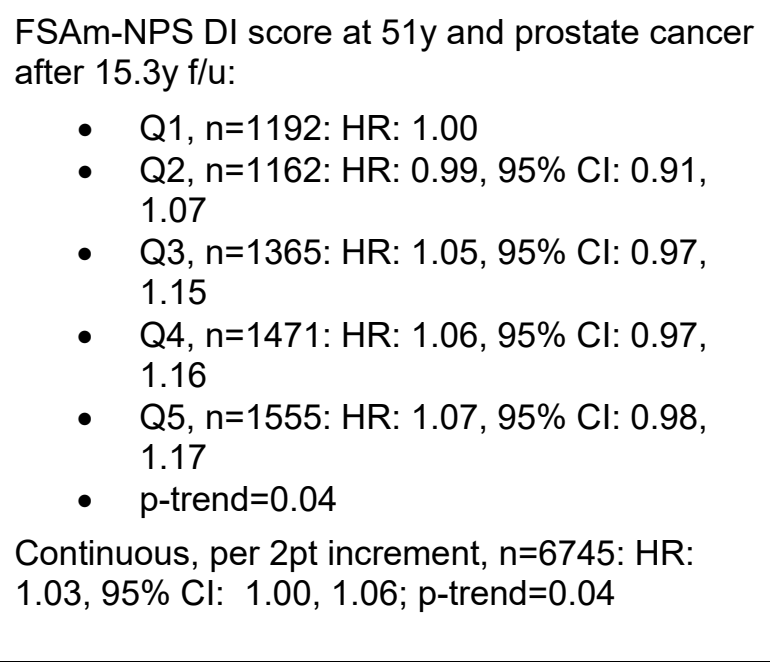 & \\
\hline
\end{tabular}

Ixxxv Abbreviations: AHEI-2010, Alternative Healthy Eating Index-2010; aMED, Alternative Mediterranean Diet Score ; BMI, body mass index; d, day; DP, Dietary pattern; \%E, \% of energy; EPIC, European Prospective Investigation into Cancer and Nutrition; FFQ, food frequency questionnaire; FSAm-NPS, Nutrient Profiling System of the British Food Standards Agency (modified version); FSAm-NPS DI, Nutrient Profiling System of the British Food Standards Agency dietary index; f/u, follow-up; HR, hazard ratio; MEDI-LITE, Mediterranean diet score; MDS, Mediterranean Diet Score; mMED, modified Mediterranean diet scores; mMEDr, modified Mediterranean diet scores without alcohol; mo, month(s); N/A, Not applicable; NIH, National Institutes of Health; NOVA, Ultra-processed food score; NS, Not significant; NR, Not reported; PCS, prospective cohort study; PNNS-GS, French National Nutrition Health Program-Guideline Score; pt, point; RCT, randomized controlled trial; WCRF/AICR, World Cancer Research Fund/American Institute for Cancer Research; wk, week(s); y, year(s) 


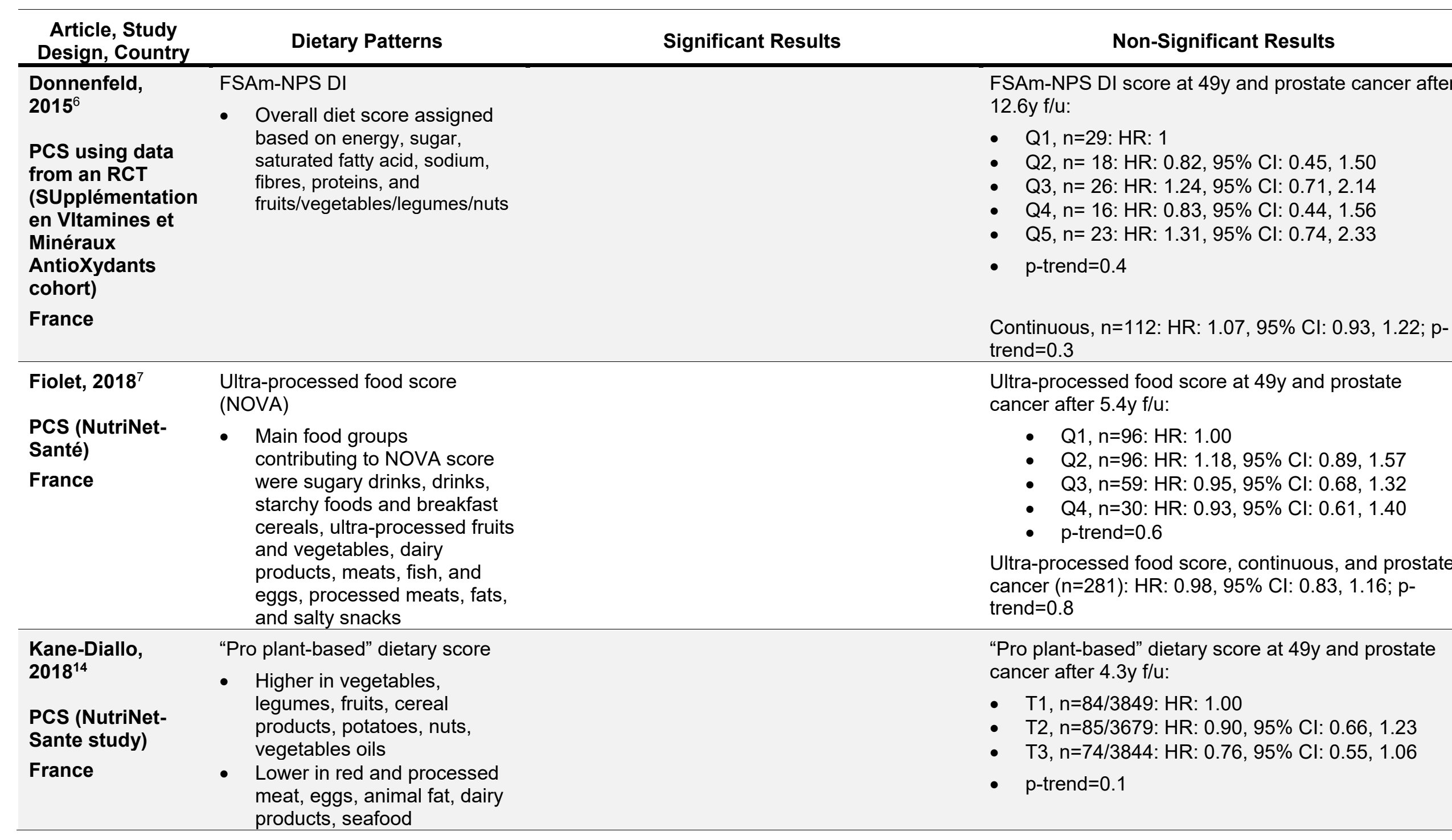




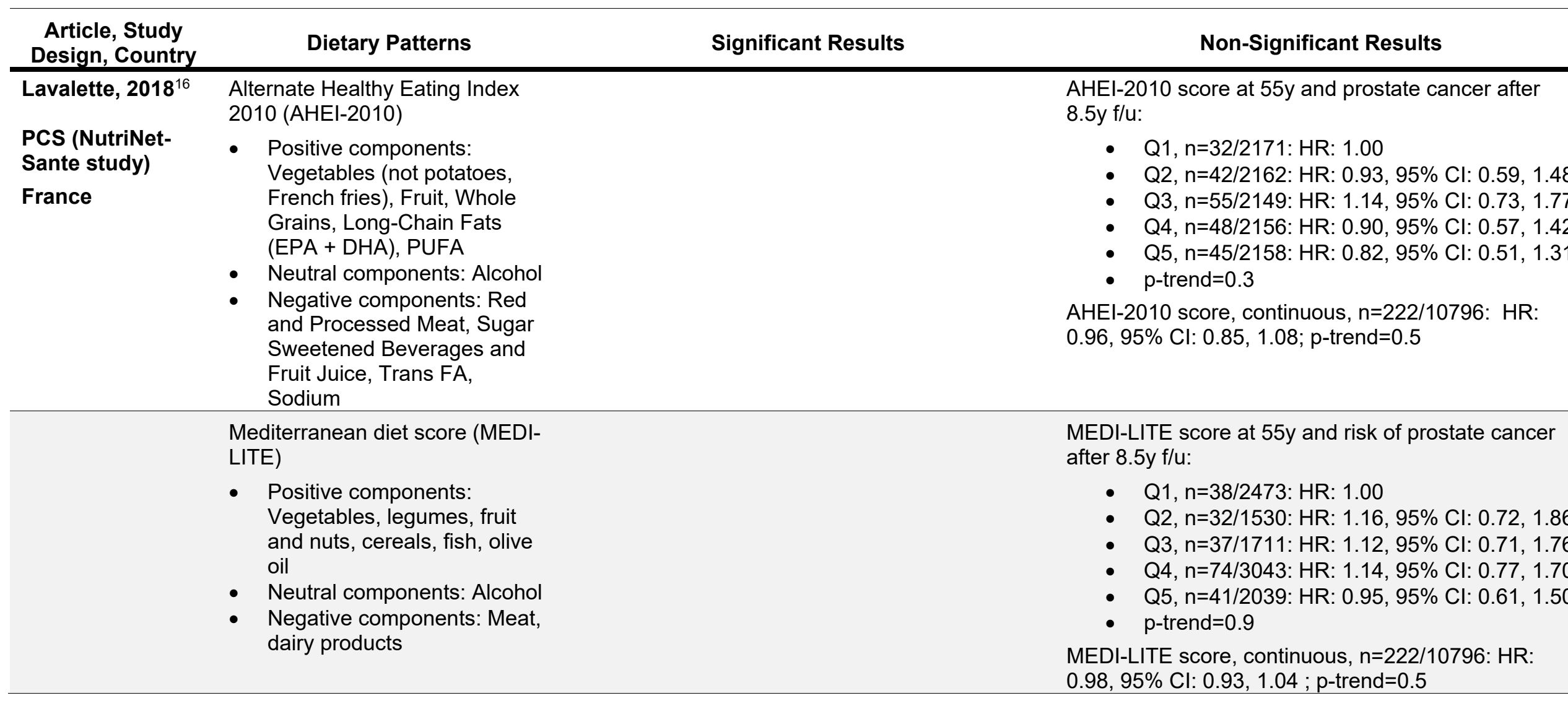




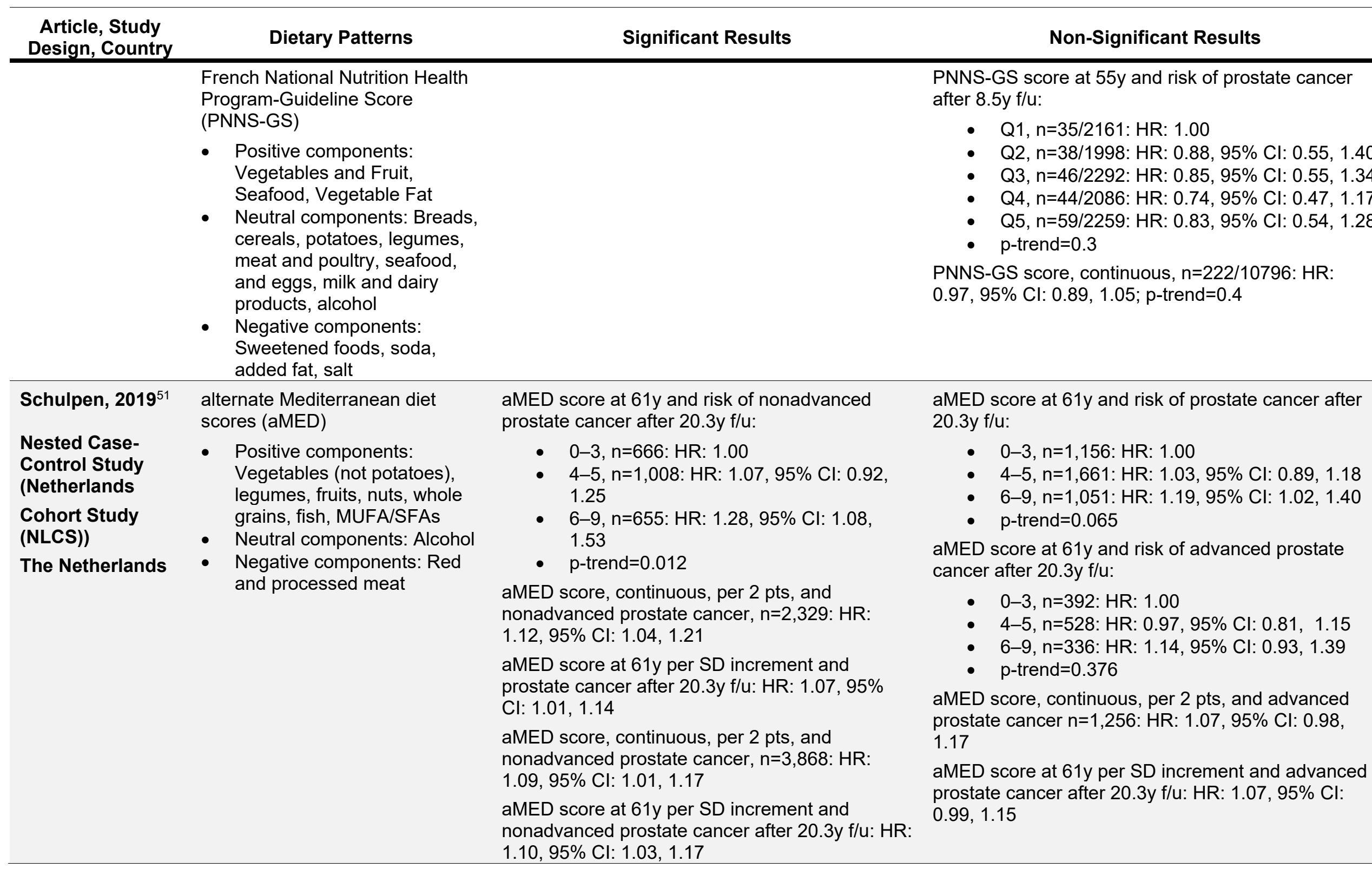




\begin{tabular}{|c|c|c|c|}
\hline $\begin{array}{c}\text { Article, Study } \\
\text { Design, Country }\end{array}$ & Dietary Patterns & Significant Results & Non-Significant Results \\
\hline & aMED without alcohol (aMEDr) & 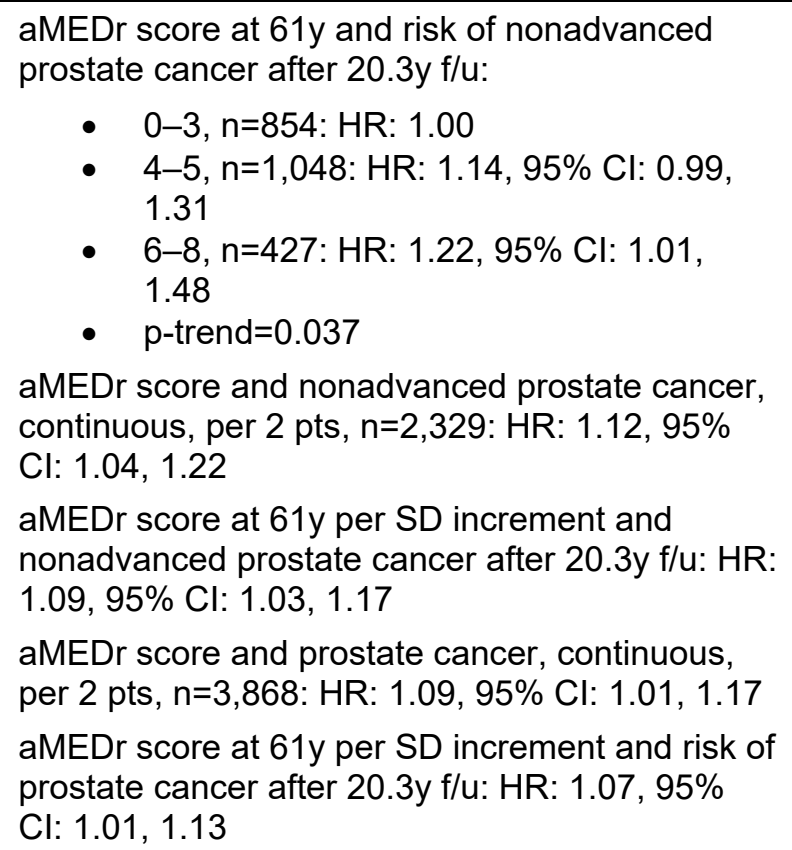 & 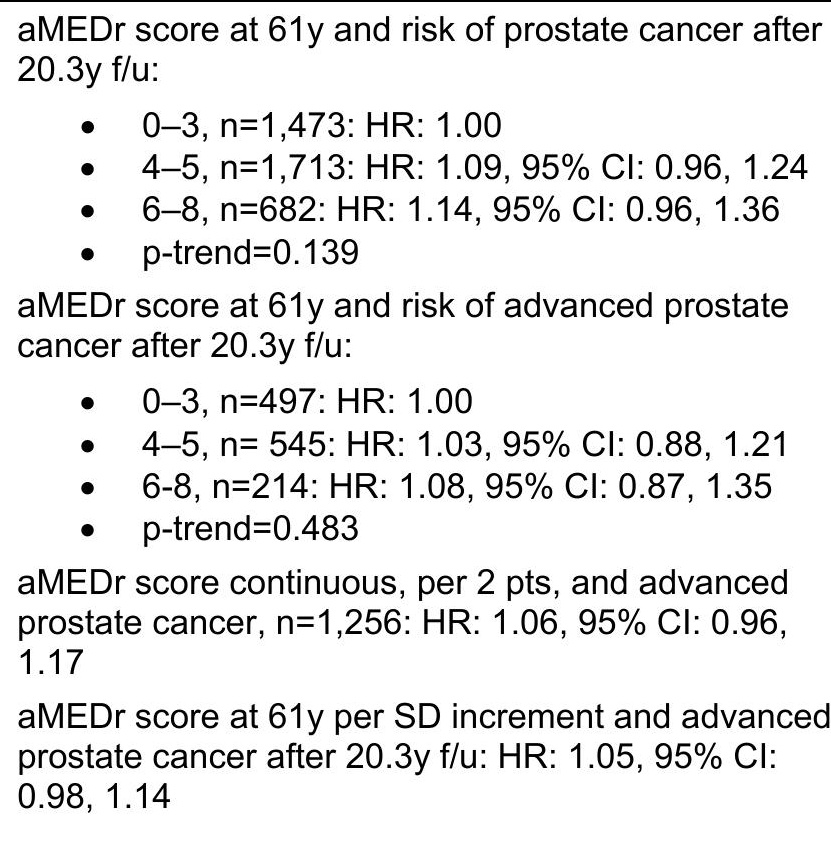 \\
\hline & $\begin{array}{l}\text { modified Mediterranean diet } \\
\text { score (mMED) } \\
\text { - } \quad \text { Positive components: } \\
\text { Vegetables, legumes, fruit, } \\
\text { cereals, fish, } \\
\text { MUFA+PUFA/SFA } \\
\text { - Neutral components: Alcohol } \\
\text { - Negative components: Meat, } \\
\text { dairy products }\end{array}$ & 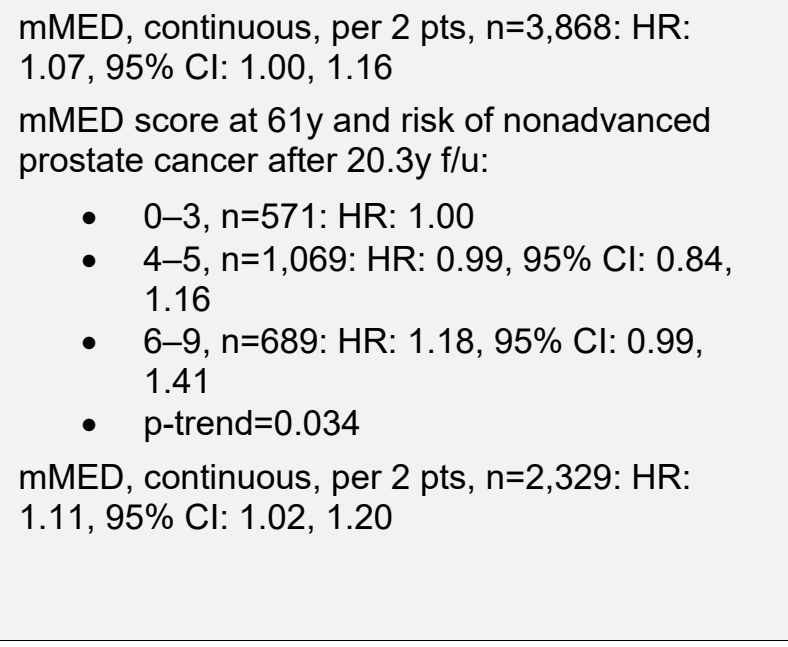 & 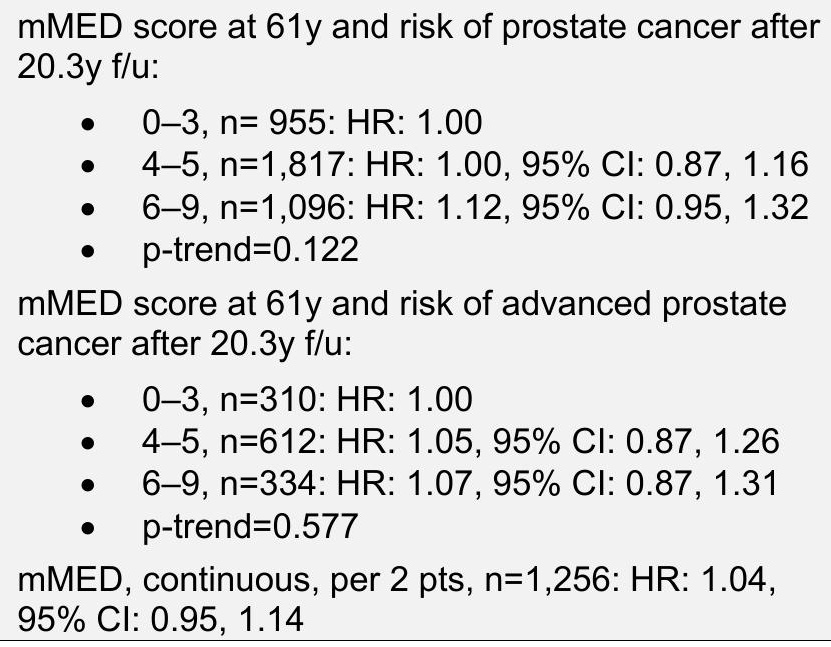 \\
\hline
\end{tabular}




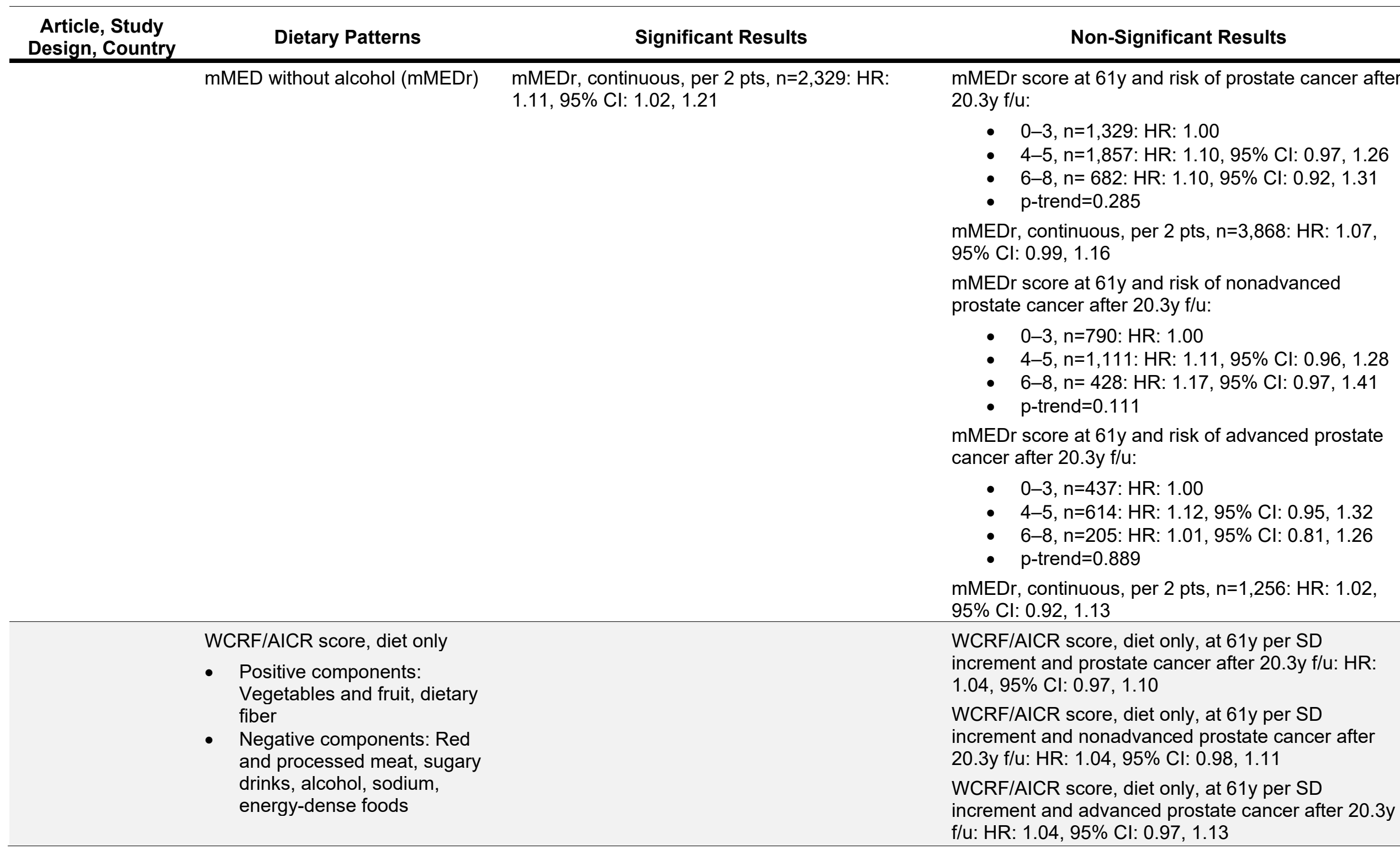




\begin{tabular}{|c|c|c|c|}
\hline $\begin{array}{l}\text { Article, Study } \\
\text { Design, Country }\end{array}$ & Dietary Patterns & Significant Results & Non-Significant Results \\
\hline & \multirow[t]{3}{*}{$\begin{array}{l}\text { WCRF/AICR score, diet only, } \\
\text { without alcohol }\end{array}$} & & $\begin{array}{l}\text { WCRF/AICR score at } 61 \mathrm{y} \text {, without alcohol, per SD } \\
\text { increment and prostate cancer after } 20.3 \mathrm{y} \text { flu: HR: } \\
1.03,95 \% \mathrm{Cl}: 0.97,1.09\end{array}$ \\
\hline & & & $\begin{array}{l}\text { WCRF/AICR score at } 61 \mathrm{y} \text {, without alcohol, per SD } \\
\text { increment and nonadvanced prostate cancer after } \\
20.3 \mathrm{y} / \mathrm{u}: \mathrm{HR}: 1.03,95 \% \mathrm{Cl}: 0.97,1.10\end{array}$ \\
\hline & & & $\begin{array}{l}\text { WCRF/AICR score at } 61 \mathrm{y} \text {, without alcohol, per SD } \\
\text { increment and advanced prostate cancer after } 20.3 \mathrm{y} \\
\text { f/u: HR: } 1.05,95 \% \mathrm{Cl}: 0.97,1.13\end{array}$ \\
\hline \multirow{6}{*}{$\begin{array}{l}\text { Shin, } 2018^{37} \\
\text { PCS (The Japan } \\
\text { Public Health } \\
\text { Center-based } \\
\text { Prospective } \\
\text { Study (JPHC)) } \\
\text { Japan }\end{array}$} & \multirow{6}{*}{$\begin{array}{l}\text { "Prudent" pattern: higher loadings } \\
\text { for vegetables, fruit, noodles, } \\
\text { potatoes, soy products, } \\
\text { mushrooms, and seaweed }\end{array}$} & & $\begin{array}{l}\text { Adherence to the "prudent" pattern at } 56 \mathrm{y} \text { and risk of } \\
\text { total prostate cancer after } 13.8 \mathrm{y} / \mathrm{u} \text { : }\end{array}$ \\
\hline & & & $\begin{array}{l}\text { - Q1, } n=193: H R: 1.00 \\
\text { - Q2, } n=185: H R: 0.91,95 \% \mathrm{Cl}: 0.74,1.12 \\
\text { - Q4, } n=242: \text { HR: } 1.05,95 \% \text { Cl: } 0.87,1.27 \\
\text { - } \quad \text { Q5, } n=281: \text { HR: } 1.01,95 \% \text { Cl: } 0.84,1.23 \\
\text { p-trend=0.715 }\end{array}$ \\
\hline & & & $\begin{array}{l}\text { Adherence to the "prudent" pattern at } 56 \mathrm{y} \text { and risk of } \\
\text { localized prostate cancer after } 13.8 \mathrm{y} \text { f/u: }\end{array}$ \\
\hline & & & $\begin{array}{l}\text { - Q1, } n=123: H R: 1.00 \\
\text { - Q2, } n=128: H R: 0.98,95 \% \text { Cl: } 0.77,1.26 \\
\text { - } Q 4, n=177: H R: 1.19,95 \% \text { Cl: } 0.94,1.50 \\
\text { - } Q 5, n=190: H R: 1.15,95 \% \text { Cl: } 0.91,1.45 \\
\text { p-trend=0.555 }\end{array}$ \\
\hline & & & $\begin{array}{l}\text { Adherence to the "prudent" pattern at } 56 \mathrm{y} \text { and risk of } \\
\text { advanced prostate cancer after } 13.8 \mathrm{y} / \mathrm{u} \text { : }\end{array}$ \\
\hline & & & $\begin{array}{l}\text { - } \quad \text { Q1, } n=59: \text { HR: } 1.00 \\
\text { - } \quad \text { Q2, } n=50: \text { HR: } 0.82,95 \% \mathrm{Cl}: 0.56,1.21 \\
\text { - } \quad \text { Q4, } n=57: \text { HR: } 0.80,95 \% \mathrm{Cl}: 0.55,1.16 \\
\text { - } \quad \text { Q5, } n=73: \text { HR: } 0.78,95 \% \mathrm{Cl}: 0.52,1.10 \\
\text { - } \text {-trend=0.135 }\end{array}$ \\
\hline
\end{tabular}




\begin{tabular}{|c|c|c|c|}
\hline $\begin{array}{c}\text { Article, Study } \\
\text { Design, Country }\end{array}$ & Dietary Patterns & Significant Results & Non-Significant Results \\
\hline & $\begin{array}{l}\text { "Westernized" pattern: higher } \\
\text { loadings for meat and processed } \\
\text { meat, eel, dairy products, fruit } \\
\text { juice, coffee, tea, soft drink, } \\
\text { sauces, and alcohol }\end{array}$ & 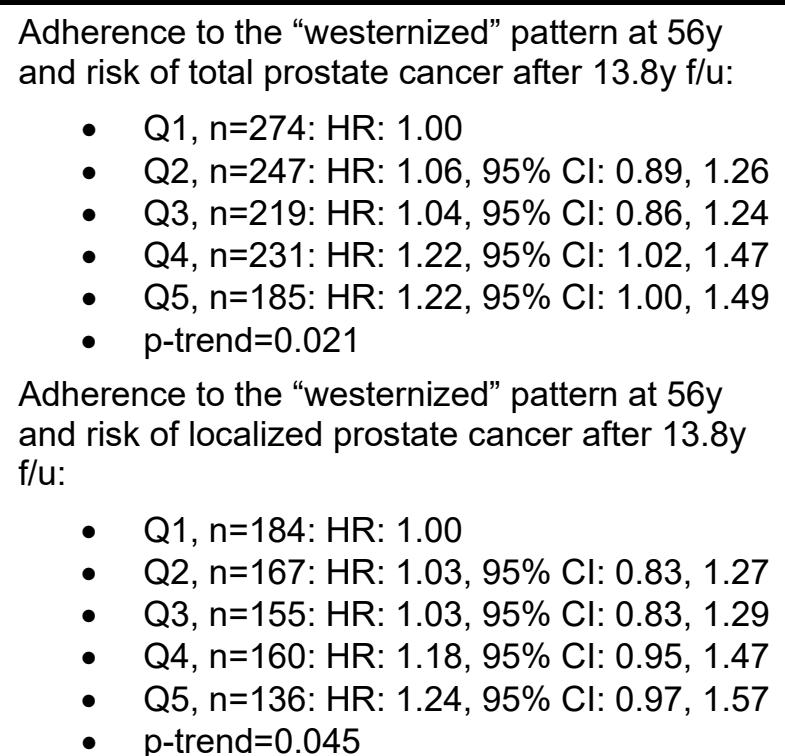 & $\begin{array}{l}\text { Adherence to the "westernized" pattern at } 56 \mathrm{y} \text { and } \\
\text { risk of advanced prostate cancer after } 13.8 \mathrm{y} \text { f/u: } \\
\text { - Q1, } \mathrm{n}=73: \mathrm{HR}: 1.00 \\
\text { - Q2, n=65: HR: } 1.12,95 \% \mathrm{Cl}: 0.80,1.57 \\
\text { - Q3, n=56: HR: } 1.09,95 \% \mathrm{Cl}: 0.76,1.56 \\
\text { - Q4, n=56: HR: } 1.25,95 \% \mathrm{Cl}: 0.87,1.81 \\
\text { - } \quad \mathrm{p} \text {-trend=0. } \mathrm{HR}: 1.23,95 \% \mathrm{Cl}: 0.82,1.84\end{array}$ \\
\hline
\end{tabular}




\begin{tabular}{|c|c|c|c|}
\hline $\begin{array}{l}\text { Article, Study } \\
\text { Design, Country }\end{array}$ & Dietary Patterns & Significant Results & Non-Significant Results \\
\hline & \multirow{6}{*}{$\begin{array}{l}\text { "Traditional" pattern: higher } \\
\text { loadings for pickles, seafood, fish } \\
\text { (oily, salty, and lean fish, and } \\
\text { salmon), chicken, and sake }\end{array}$} & & $\begin{array}{l}\text { Adherence to the "traditional" pattern at } 56 \mathrm{y} \text { and risk } \\
\text { of total prostate cancer after } 13.8 \mathrm{y} / \mathrm{u} \text { : }\end{array}$ \\
\hline & & & 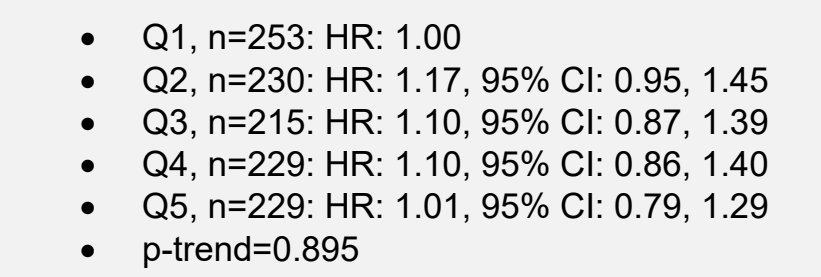 \\
\hline & & & $\begin{array}{l}\text { Adherence to the "traditional" pattern at } 56 \mathrm{y} \text { and risk } \\
\text { of localized prostate cancer after } 13.8 \mathrm{y} / \mathrm{u} \text { : }\end{array}$ \\
\hline & & & $\begin{array}{l}\text { - } Q 1, n=182: H R: 1.00 \\
\text { - } Q 2, n=146: H R: 1.08,95 \% \mathrm{Cl}: 0.83,1.40 \\
\text { - } Q 4, n=147: H R: 1.06,95 \% \mathrm{Cl}: 0.80,1.41 \\
\text { - } Q 5, n=160: H R: 1.10,95 \% \mathrm{Cl}: 0.82,1.48 \\
\text { - } \text { p-trend=0.869 }\end{array}$ \\
\hline & & & $\begin{array}{l}\text { Adherence to the "traditional" pattern at } 56 \mathrm{y} \text { and risk } \\
\text { of advanced prostate cancer after } 13.8 \mathrm{y} \text { f/u: }\end{array}$ \\
\hline & & & 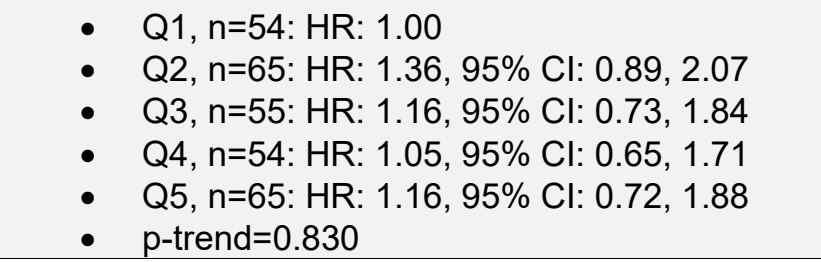 \\
\hline
\end{tabular}




\begin{tabular}{|c|c|c|c|}
\hline $\begin{array}{c}\text { Article, Study } \\
\text { Design, Country }\end{array}$ & Dietary Patterns & Significant Results & Non-Significant Results \\
\hline $\begin{array}{l}\text { Tantamango- } \\
\text { Bartley, 2016 } \\
\\
\text { PCS (Adventist } \\
\text { Health Study-2 } \\
\text { (AHS-2)) } \\
\text { United States }\end{array}$ & $\begin{array}{l}\text { "Vegetarian" dietary pattern: } \\
\text { "Vegans": Red meat, poultry, } \\
\text { fish; eggs; and dairy <1 } \\
\text { time/mo } \\
\text { "Lacto-ovo-vegetarian": Red } \\
\text { meat, poultry, and fish <1 } \\
\text { time/mo, eggs or dairy }>1 \\
\text { time/mo } \\
\text { "Pesco-vegetarian": Red } \\
\text { meat or poultry <1 time/mo, } \\
\text { fish }>1 \text { time/mo, and } \\
\text { eggs/dairy in any amount } \\
\text { "Semi-vegetarian": Red meat } \\
\text { or poultry }>1 \text { time/mo, and all } \\
\text { meats combined (including } \\
\text { fish) }<1 \text { time/wk and } \\
\text { eggs/dairy in any amount } \\
\text { "Nonvegetarians": Red meat } \\
\text { and poultry }>1 \text { time/mo and } \\
\text { all meats combined } \\
\text { (including fish) }>1 \text { time/wk, } \\
\text { and eggs/dairy in any amount } \\
\text { "Vegetarians" vs. } \\
\text { "nonvegetarians" consumed } \\
\text { higher amounts of fruits, } \\
\text { vegetables, avocados, non-fried } \\
\text { potatoes, whole grains, legumes, } \\
\text { soy foods, nuts and seeds, and } \\
\text { was observed among } \\
\text { vegetarians; and lower amounts } \\
\text { of meats, dairy products, eggs, } \\
\text { refined grains, added fats, } \\
\text { sweets, snack foods and non- } \\
\text { water beverages }\end{array}$ & $\begin{array}{l}\text { Dietary pattern at }>30 \mathrm{y} \text { and risk of overall } \\
\text { prostate cancer after } 7.8 \mathrm{y} \text { f/u: } \\
\text { - "Vegan", n=59: HR: } 0.66,95 \% \mathrm{Cl}: 0.50,0.87 \\
\text { - "Lacto-ovo-vegetarian", n=333: HR: } 0.96, \\
95 \% \mathrm{Cl}: 0.83,1.12 \\
\text { - "Pesco-vegetarian", n=121: HR: } 1.07,95 \% \\
\mathrm{Cl}: 0.88,1.31 \\
\text { - "Semi-vegetarian", n=63: HR: } 1.18,95 \% \mathrm{Cl} \text { : } \\
0.91,1.54 \\
\text { - "Nonvegetarian", n=503: HR: } 1.00 \\
\text { When results were stratified by race, results were } \\
\text { only significant in white subjects. }\end{array}$ & 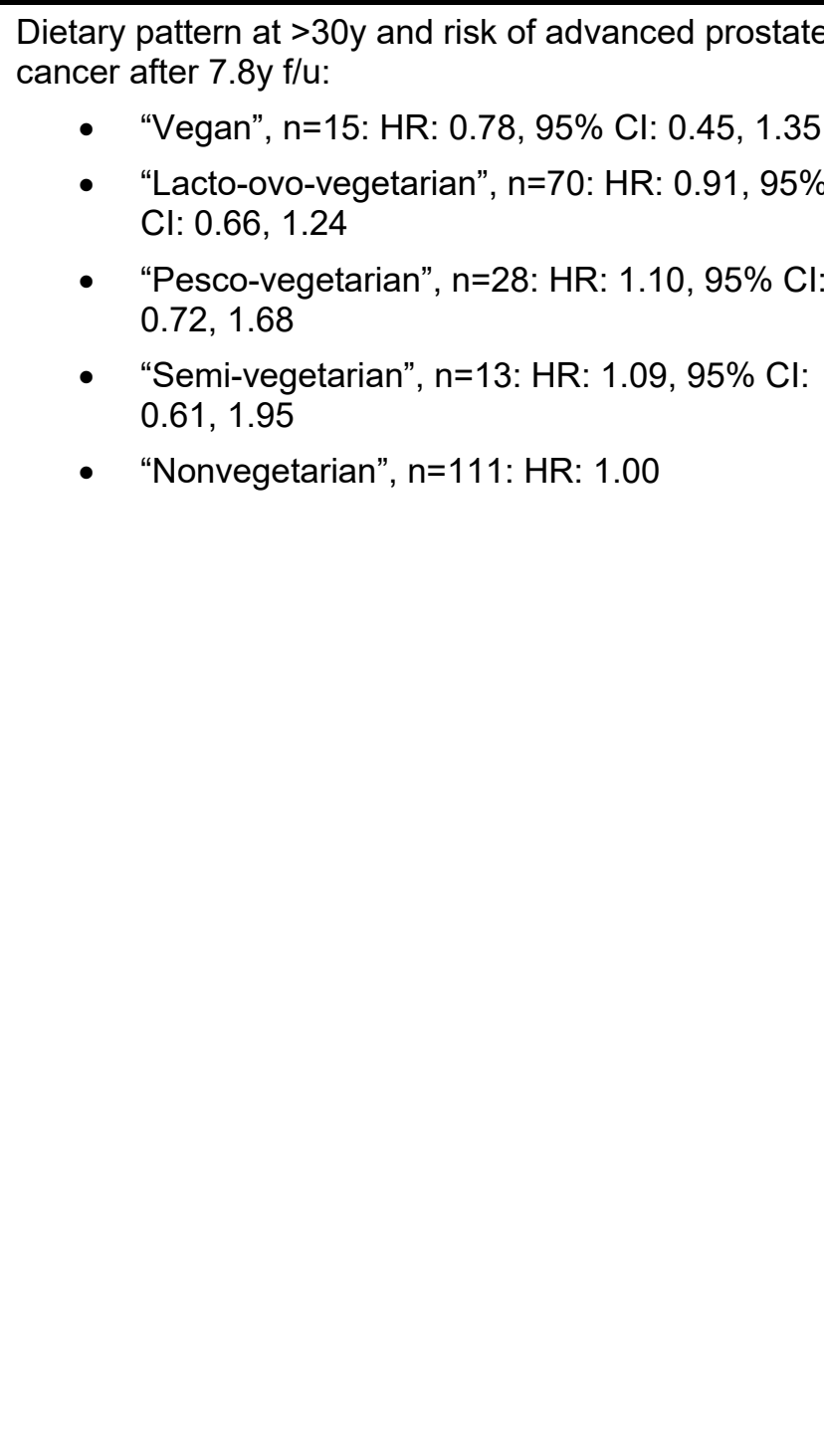 \\
\hline
\end{tabular}


Table 14. Risk of bias for observational studies examining dietary patterns and prostate cancer|xxxvi,Ixxxvii

\begin{tabular}{|c|c|c|c|c|c|c|c|}
\hline & Confounding & $\begin{array}{l}\text { Selection of } \\
\text { participants }\end{array}$ & $\begin{array}{l}\text { Classification } \\
\text { of exposures }\end{array}$ & $\begin{array}{c}\text { Deviations } \\
\text { from intended } \\
\text { exposures }\end{array}$ & Missing data & $\begin{array}{c}\text { Outcome } \\
\text { measurement }\end{array}$ & $\begin{array}{l}\text { Selection of the } \\
\text { reported result }\end{array}$ \\
\hline Deschasaux, $2017^{5}$ & Serious & Moderate & Low & Serious & Moderate & Low & Moderate \\
\hline Donnenfeld, $2015^{6}$ & Serious & Moderate & Low & Serious & Moderate & Low & Moderate \\
\hline Fiolet, $2018^{7}$ & Serious & Moderate & Moderate & Serious & Moderate & Low & Moderate \\
\hline Kane-Diallo, 2018 ${ }^{14}$ & Serious & Moderate & Low & Serious & Moderate & Low & Moderate \\
\hline Lavalette, $2018^{16}$ & Serious & Moderate & Low & Serious & Moderate & Low & Moderate \\
\hline Schulpen, $2019^{51}$ & Serious & Serious & Low & Serious & Moderate & Low & Serious \\
\hline Shin, $2018^{37}$ & Serious & Moderate & Moderate & Serious & Moderate & Low & Moderate \\
\hline $\begin{array}{l}\text { Tantamango-Bartley, } \\
2016^{50}\end{array}$ & Serious & Moderate & Low & Moderate & Serious & Low & Moderate \\
\hline
\end{tabular}

Ixxxvi A detailed description of the methodology used for assessing risk of bias is available on the NESR website: https://nesr.usda.gov/2020-dietaryguidelines-advisory-committee-systematic-reviews and in Part C of the following reference: Dietary Guidelines Advisory Committee. 2020. Scientific Report of the 2020 Dietary Guidelines Advisory Committee: Advisory Report to the Secretary of Agriculture and the Secretary of Health and Human Services. U.S. Department of Agriculture, Agricultural Research Service, Washington, DC.

Ixxxvii Possible ratings of low, moderate, serious, critical, or no information determined using the "Risk of Bias for Nutrition Observational Studies" tool (RoBNObs) (Dietary Guidelines Advisory Committee. 2020. Scientific Report of the 2020 Dietary Guidelines Advisory Committee: Advisory Report to the Secretary of Agriculture and the Secretary of Health and Human Services. U.S. Department of Agriculture, Agricultural Research Service, Washington, DC.) 
The NESR team used its rigorous, protocol-driven methodology to support the 2020 Dietary Guidelines Advisory Committee in conducting this update to an existing systematic review.

NESR's systematic review methodology involves:

- Developing a protocol,

- Searching for and selecting studies,

- Extracting data from and assessing the risk of bias of each included study,

- Synthesizing the evidence,

- Developing conclusion statements,

- Grading the evidence underlying the conclusion statement, and

- Recommending future research.

A detailed description of the methodology used in conducting this systematic review is available on the NESR website: https://nesr.usda.gov/2020-dietary-guidelines-advisory-committee-systematicreviews, and can be found in the 2020 Dietary Guidelines Advisory Committee Report, Part C: Methodology. Ixxxviii This systematic review was peer reviewed by Federal scientists, and information about the peer review process can also be found in the Committee's Report, Part C. Methodology. Additional information about this systematic review, including a description of and rationale for any modifications made to the protocol can be found in the 2020 Dietary Guidelines Advisory Committee Report, Part D: Chapter 8. Dietary Patterns.

The systematic review described in this document updates existing systematic reviews that were conducted by the 2015 Dietary Guidelines Advisory Committee with support from USDA's Nutrition Evidence Systematic Review (NESR) team. Information about the 2015 Dietary Guidelines Advisory Committee's review of the evidence on dietary patterns and cancer can be found in their report, which is available at the following website: https://nesr.usda.gov/dietary-patterns-foods-and-nutrients-andhealth-outcomes-subcommittee and https://www.dietaryguidelines.gov/current-dietaryguidelines/process-develop-2015-2020-dg/advisory-committee

Below are details of the final protocol for the systematic review described herein, including the:

- Analytic framework

- Literature search and screening plan

- Literature search and screening results

\section{ANALYTIC FRAMEWORK}

The analytic framework (Figure 1) illustrates the overall scope of the systematic review, including the population, the interventions and/or exposures, comparators, and outcomes of interest. It also includes definitions of key terms and identifies key confounders and other factors to be considered in the systematic review. The inclusion and exclusion criteria that follow provide additional information about how parts of the analytic framework were defined and operationalized for the review.

Ixxxviii Dietary Guidelines Advisory Committee. 2020. Scientific Report of the 2020 Dietary Guidelines Advisory Committee: Advisory Report to the Secretary of Agriculture and the Secretary of Health and Human Services. U.S. Department of Agriculture, Agricultural Research Service, Washington, DC. 


\section{Figure 1: Analytic framework}

\section{Systematic review question: What is the relationship between dietary patterns consumed and risk of certain types}

\section{of cancer?}

\section{Intervention/exposure}

Consumption of and/or

adherence to a dietary

pattern

Population: Children through older adults; healthy and/or at risk of chronic disease

\section{Comparator}

- Consumption of and/or adherence to a different dietary pattern

- Different levels of consumption of and/or adherence to a dietary pattern

\section{Endpoint outcome(s)}

Incident cases of:

- Breast cancer

- Colorectal cancer

- Prostate cancer

- Lung cancer

Population: Children through older adults

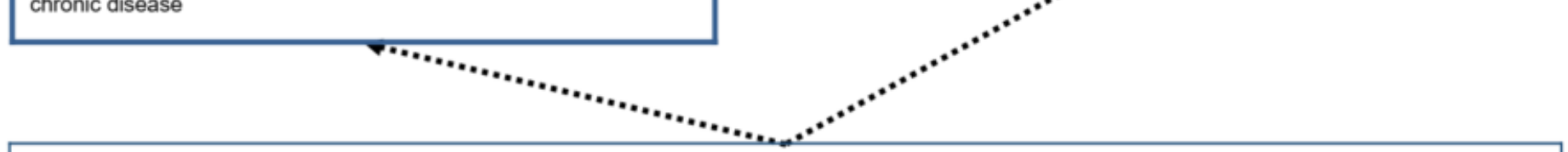

Key Confounders: Sex, Age, Race/ethnicity, Socioeconomic status, Alcohol intake (in adults), Physical activity, Smoking, Anthropometry, Family history of the cancer outcome, Hormonal contraceptive (Breast), Menopausal status (Breast), Inflammatory bowel disease (Colon and rectum), Colorectal polyps (Colon and rectum), Lung disease (Lung), Environmental exposures to lung carcinogens (Lung)

Other Factors to be Considered: Total energy intake

\section{Key definitions}

Dietary patterns - The quantities, proportions, variety, or combination of different foods, drinks, and nutrients (when available) in diets, and the frequency with which they are habitually consumed.

\section{Legend}

$\longrightarrow$ The relationship of interest in the systematic review

$\ldots \ldots \ldots$ Factors that may impact the relationship of interest in the systematic review 


\section{LITERATURE SEARCH AND SCREENING PLAN}

\section{Inclusion and exclusion criteria}

This table provides the inclusion and exclusion criteria for this update to systematic reviews. The inclusion and exclusion criteria are a set of characteristics used to determine which articles identified in the literature search were included in or excluded from the systematic review.

\section{Table 15. Inclusion and exclusion criteria}

\begin{tabular}{|c|c|c|}
\hline Category & Inclusion Criteria & Exclusion Criteria \\
\hline Study design & $\begin{array}{l}\text { - } \quad \text { Randomized controlled trials } \\
\text { - } \quad \text { Non-randomized controlled trials, including } \\
\text { quasi-experimental and controlled before } \\
\text { and after studies } \\
\text { - } \quad \text { Prospective cohort studies } \\
\text { - } \quad \text { Retrospective cohort studies } \\
\text { - } \quad \text { Nested case-control studies }\end{array}$ & $\begin{array}{l}\text { - Uncontrolled trials } \\
\text { - } \text { Cross-sectional studies } \\
\text { - Uncontrolled before-and-after studies } \\
\text { - } \quad \text { Narrative reviews } \\
\text { - } \quad \text { Systematic reviews } \\
\text { - } \quad \text { Meta-analyses }\end{array}$ \\
\hline $\begin{array}{l}\text { Intervention/ } \\
\text { exposure }\end{array}$ & $\begin{array}{l}\text { Studies that examine consumption of and/or } \\
\text { adherence to a dietary pattern [i.e., the } \\
\text { quantities, proportions, variety, or combination } \\
\text { of different foods, drinks, and nutrients (when } \\
\text { available) in diets, and the frequency with } \\
\text { which they are habitually consumed], } \\
\text { including, at a minimum, a description of the } \\
\text { foods and beverages in the pattern } \\
\text { - Dietary patterns may be measured or } \\
\text { derived using a variety of approaches, } \\
\text { such as adherence to a priori patterns } \\
\text { (indices/scores), data driven patterns } \\
\text { (factor or cluster analysis), reduced rank } \\
\text { regression, or other methods, including } \\
\text { clinical trials }\end{array}$ & $\begin{array}{l}\text { Studies that do not provide a description of the } \\
\text { dietary pattern, which at minimum, must } \\
\text { include the foods and beverages in the pattern } \\
\text { (i.e., studies that examine a labeled dietary } \\
\text { patterns, but do not describe the foods and } \\
\text { beverages consumed) }\end{array}$ \\
\hline Comparator & $\begin{array}{l}\text { Dietary patterns described by foods and } \\
\text { beverages consumed: } \\
\text { - Consumption of and/or adherence to a } \\
\text { different dietary pattern } \\
\text { - Different levels of consumption of and/or } \\
\text { adherence to a dietary pattern }\end{array}$ & - N/A \\
\hline Outcomes & $\begin{array}{l}\text { Incident cases of: } \\
\text { - } \text { Breast cancer } \\
\text { - } \text { Colorectal cancer } \\
\text { - } \quad \text { Lung cancer } \\
\text { - } \quad \text { Prostate cancer }\end{array}$ & $\begin{array}{l}\text { - Studies that exclusively examine cancer- } \\
\text { related mortality, prevalence, survivorship, } \\
\text { or recurrence of cancer }\end{array}$ \\
\hline
\end{tabular}




\begin{tabular}{|c|c|c|}
\hline Category & Inclusion Criteria & Exclusion Criteria \\
\hline Date of publication & $\begin{array}{l}\text { January } 2014 \text { - January } 2020 \text { (this date } \\
\text { range is in addition to the original } \\
\text { systematic review, which included articles } \\
\text { published from January } 2000 \text { - January } \\
2014 \text { ) }\end{array}$ & $\begin{array}{l}\text { - Articles published prior to January } 2000 \text { or } \\
\text { after January } 2020\end{array}$ \\
\hline Publication status & Articles that have been peer-reviewed & $\begin{array}{l}\text { Articles that have not been peer-reviewed and } \\
\text { are not published in peer-reviewed journals, } \\
\text { including unpublished data, manuscripts, } \\
\text { reports, abstracts, and conference } \\
\text { proceedings }\end{array}$ \\
\hline $\begin{array}{l}\text { Language of } \\
\text { publication }\end{array}$ & Articles published in English & $\begin{array}{l}\text { Articles published in languages other than } \\
\text { English }\end{array}$ \\
\hline Country ${ }^{1 \times x x i x}$ & $\begin{array}{l}\text { Studies conducted in countries ranked as high } \\
\text { or higher human development }\end{array}$ & $\begin{array}{l}\text { Studies conducted in countries ranked as } \\
\text { medium or lower human development }\end{array}$ \\
\hline Study participants & $\begin{array}{l}\text { - } \text { Human participants } \\
\text { - } \text { Males } \\
\text { - Females }\end{array}$ & $\begin{array}{l}\text { - Non-human participants (e.g., animal or in- } \\
\text { vitro models) }\end{array}$ \\
\hline \multirow[t]{2}{*}{$\begin{array}{l}\text { Age of study } \\
\text { participants }\end{array}$} & $\begin{array}{l}\text { - Age at intervention or exposure: } \\
\quad \text { Children and adolescents (ages 2-18 } \\
\text { years) } \\
\circ \text { Adults (ages 19-64 years) } \\
\circ \text { Older adults (ages } 65 \text { years and older) }\end{array}$ & $\begin{array}{l}\text { - Age at intervention or exposure: } \\
\circ \quad \text { Infants and toddlers (birth to } 24 \\
\text { months) }\end{array}$ \\
\hline & $\begin{array}{l}\text { - Age at outcome: } \\
\quad \text { Children and adolescents (ages 2-18 } \\
\text { years) } \\
\circ \text { Adults (ages 19-64 years) } \\
\circ \text { Older adults (ages } 65 \text { years and older) }\end{array}$ & $\begin{array}{l}\text { - Age at outcome: } \\
\text { Infants and toddlers (birth to } 24 \\
\text { months) }\end{array}$ \\
\hline
\end{tabular}

Ixxxix The Human Development classification was based on the Human Development Index (HDI) ranking from the year the study intervention occurred or data were collected (UN Development Program. HDI 1990-2017 HDRO calculations based on data from UNDESA (2017a), UNESCO Institute for Statistics (2018), United Nations Statistics Division (2018b), World Bank (2018b), Barro and Lee (2016) and IMF (2018). Available from:

http://hdr.undp.org/en/data). If the study did not report the year in which the intervention occurred or data were collected, the HDI classification for the year of publication was applied. HDI values are available from 1980, and then from 1990 to present. If a study was conducted prior to 1990, the HDI classification from 1990 was applied. If a study was conducted in 2018 or 2019 , the most current HDI classification was applied. When a country was not included in the HDI ranking, the current country classification from the World Bank was used instead (The World Bank. World Bank country and lending groups. Available from: https://datahelpdesk.worldbank.org/knowledgebase/articles/906519-world- country-and-lending-groups). 


\begin{tabular}{lll}
\hline Category & Inclusion Criteria & Exclusion Criteria \\
\hline $\begin{array}{l}\text { Health status of } \\
\text { study } \\
\text { participants }\end{array}$ & $\begin{array}{l}\text { Studies that enroll participants who are } \\
\text { healthy and/or at risk for chronic } \\
\text { disease, including those with obesity }\end{array}$ & $\begin{array}{l}\text { Studies that exclusively enroll participants } \\
\text { diagnosed with a disease, or hospitalized } \\
\text { patients with illness or injury. (For this } \\
\text { criterion, studies that exclusively enroll } \\
\text { subjects with obesity will be included.) }\end{array}$ \\
& $\begin{array}{l}\text { Studies that enroll some participants } \\
\text { diagnosed with a disease }\end{array}$ & $\begin{array}{l}\text { Studies that exclusively enroll participants } \\
\text { with cancer (i.e., studies that aim to treat } \\
\text { participants who have already been diagnosed } \\
\text { with the outcome of interest) }\end{array}$ \\
\hline
\end{tabular}

\section{Electronic databases and search terms}

\section{PubMed}

- Provider: U.S. National Library of Medicine

- Date(s) Searched: January 13, 2020

- Date range searched: January 1, 2014-January 13, 2020

- Search Terms:

\#1 - dietary pattern* OR diet pattern* OR eating pattern* OR food pattern* OR diet quality* OR eating habit ${ }^{*}$ OR dietary habit* OR diet habit* OR food habit* OR beverage habit* OR "Feeding Behavior"[Mesh:NoExp] OR dietary profile* OR food profile* OR diet profile* OR eating profile* OR dietary guideline ${ }^{*}$ OR dietary recommendation* OR dietary intake* OR eating style* OR "Diet, Mediterranean"[Mesh] OR Mediterranean Diet*[tiab] OR "Dietary Approaches To Stop Hypertension"[Mesh] OR Dietary Approaches To Stop Hypertension Diet* OR DASH $\operatorname{diet}^{*}$ OR "Diet, Gluten-Free"[Mesh] OR Gluten Free diet* OR prudent diet* OR "Diet, Paleolithic"[Mesh] OR Paleolithic Diet ${ }^{*}$ OR "Diet, Vegetarian"[Mesh] OR vegetarian $\operatorname{diet}^{\star}\left[\right.$ tiab] OR vegan $\operatorname{diet}^{*}$ OR "Diet, Healthy"[Mesh] OR plant based diet* OR "Diet, Western"[Mesh] OR western diet* OR "Diet, Carbohydrate-Restricted"[Mesh] OR low-carbohydrate diet* OR high carbohydrate diet* OR Ketogenic Diet* OR Nordic Diet* OR "Diet, Fat-Restricted"[Mesh] OR "Diet, HighFat"[Mesh] OR "Diet, High-Protein"[Mesh] OR high protein diet*'tiab] OR protein intake* OR high-fat diet* OR low fat diet* OR "Diet, Protein-Restricted"[Mesh] OR low protein diet $^{\star}$ OR "Diet, Sodium-Restricted"[Mesh] OR low-sodium diet* OR low salt diet* OR (("Dietary Proteins"[Mesh] OR dietary protein*[tiab] OR "Dietary Carbohydrates"[Mesh] OR dietary carbohydrate*[tiab] OR "Dietary Fats"[Mesh] OR dietary fat*[tiab] OR hypocaloric OR hypocaloric) AND (diet[tiab] OR diets[tiab] OR consumption[tiab] OR intake[tiab] OR supplement*[tiab])) OR ((“Guideline Adherence"[Mesh] OR guideline adherence*)AND (diet[tiab] OR dietary[tiab] OR food[tiab] OR beverage*[tiab] OR nutrition*[tiab])) OR diet score* OR diet quality score* OR diet quality index* OR kidmed OR diet index* OR dietary index* OR food score* OR MedDietScore OR healthy eating index[tiab] OR ((pattern[tiab] OR patterns[tiab] OR consumption[tiab] OR habit"[tiab]) AND ("Diet"[Mesh:NoExp] OR diet[tiab] OR diets[tiab] OR dietary[tiab] OR "Food"[Mesh] OR food[tiab] OR foods[tiab] OR "Beverages"[Mesh] OR beverage[tiab] OR beverages[tiab]))

\# 2- "Breast Neoplasms"[Mesh] OR breast neoplasm* OR breast cancer*[tiab] OR breast carcino* OR "Colorectal Neoplasms"[Mesh] OR colorectal neoplasm* OR colorectal cancers* 
OR colorectal carcino* OR colon neoplasm* OR colon cancer* OR colon carcino* OR "Intestinal Polyps"[Mesh] OR intestinal polyp* OR colonic polyp* OR colorectal polyp* OR colorectal lesion* OR rectal neoplasm* OR rectal cancer* OR rectal carcino* OR "Prostatic Neoplasms"[Mesh] OR prostate neoplasm* OR prostate cancer* OR prostate carcino* OR "Lung Neoplasms"[Mesh] OR lung neoplasm* OR lung carcino* OR lung cancer* OR "Liver Neoplasms"[Mesh] OR liver neoplasm* OR liver cancer* OR hepatic neoplasm* OR hepatic cancer $^{*}$ OR hepatocellular carcino* OR "Pancreatic Neoplasms"[Mesh] OR pancreatic neoplasm* OR pancreatic cancer* OR pancreatic carcino* OR pancreatic adenocarcinoma* OR pancreatic neuro* OR ampullary cancer ${ }^{*}$ OR ampullary carcino* OR exocrine cancer ${ }^{*}$ OR exocrine carcino* OR "Endometrial Neoplasms"[Mesh] OR endometrial neoplasm* OR endometrial cancer* OR endometrial carcino* OR endometrioid neoplasm* OR endometrioid cancer ${ }^{*}$ OR endometrioid carcino* OR "Endometrial Hyperplasia"[Mesh] OR endometrial hyperplasia* OR "Leukemia"[Mesh] OR leukem* OR leukaem* OR leucem* OR leucaem* OR ((cancer*[tiab] OR "Neoplasms"[Mesh] OR neoplasm*[tiab] OR carcino*[tiab] OR "Carcinogens"[Mesh] OR malignan*[tiab] OR adenocarcinoma*[tiab] OR sarcoma*[tiab] OR metastasis[tiab] OR metastases[tiab] OR tumor[tiab] OR tumors[tiab] OR tumour*[tiab] OR "Polyps"[Mesh] OR polyp[tiab] OR polyps[tiab]) AND ("Colon"[Mesh] OR colonic*[tiab] OR colon[tiab] OR colorect*[tiab] OR rectal[tiab] OR rectum[tiab] OR "Colonic Diseases"[Mesh:noexp] OR "Breast"[Mesh] OR "Breast Diseases"[Mesh:noexp] OR breast $^{*}[$ tiab] OR mammary[tiab] OR "Prostate"[Mesh] OR prostate*[tiab] OR prostatic[tiab] OR "Prostatic Diseases"[Mesh:noexp] OR "Lung"[Mesh] OR "Lung Diseases"[Mesh:noexp] OR lung[tiab] OR pulmonary[tiab] OR endometri* OR endometrium* OR "Uterine Diseases"[Mesh:noexp] OR "uterine diseases*" OR "Liver"[Mesh] OR liver[tiab] OR "Liver Diseases"[Mesh:noexp] OR "Pancreas"[Mesh] OR pancreas[tiab] OR pancreati" [tiab] OR "Pancreatic Diseases"[Mesh:noexp] OR "Ampulla of Vater"[Mesh] OR "ampulla of vater"))

\#3 - (\#1 AND \#2) NOT ("Animals"[Mesh] NOT ("Animals"[Mesh] AND "Humans"[Mesh])) NOT editorial[ptyp] OR comment[ptyp] OR news[ptyp] OR letter[ptyp] OR review[ptyp] OR systematic review[ptyp] OR systematic review[ti] OR meta-analysis[ptyp] OR meta-analysis[ti] OR meta-analyses[ti] OR retracted publication[ptyp] OR retraction of publication[ptyp] OR retraction of publication[tiab] OR retraction notice[ti] Filters: Publication date from 2014/01/01 to 2020/01/13; English

\section{Cochrane Central Register of Controlled Trials (CENTRAL)}

- Provider: John Wiley \& Sons

- Date(s) Searched: January 13, 2020

- Date range searched: January 1, 2014-January 13, 2020

- Search Terms:

\#1 - "dietary pattern*" OR "diet pattern*" OR "eating pattern*" OR "food pattern*" OR "diet quality" OR "eating habit" OR "dietary habit*" OR "diet habit" OR "food habit*" OR "beverage habit*" OR [mh ^"Feeding Behavior"] OR "feeding behavior" OR "dietary profile*" OR "food profile*" OR "diet profile*" OR "eating profile*" OR "dietary guideline*" OR "dietary recommendation" OR "dietary intake*" OR "eating style*" OR [mh "Diet, Mediterranean"] OR "Mediterranean Diet*" OR [mh "Dietary Approaches To Stop Hypertension"] OR "Dietary Approaches To Stop Hypertension Diet" OR "DASH diet*" OR [mh "Diet, Gluten-Free"] OR "Gluten Free diet*" OR "prudent diet*" OR [mh "Diet, Paleolithic"] OR "Paleolithic Diet" OR [mh "Diet, Vegetarian"] OR "vegetarian diet*" OR "vegan diet*" OR [mh "Diet, Healthy"] OR 
"healthy diet" OR "plant based diet*" OR [mh "Diet, Western"] OR "western diet*" OR [mh "Diet, Carbohydrate-Restricted"] OR "low-carbohydrate diet"" OR "high carbohydrate diet*" OR "Ketogenic Diet*" OR "Nordic Diet*" OR [mh "Diet, Fat-Restricted"] OR [mh "Diet, High-Fat"] OR [mh "Diet, High-Protein"] OR "high protein diet*" OR "protein intake*" OR "high-fat diet" OR "low fat diet*" OR [mh "Diet, Protein-Restricted"] OR "low protein diet*" OR [mh "Diet, Sodium-Restricted"] OR "low-sodium diet*" OR "low salt diet*"

\#2 - (([mh "Dietary Proteins"] OR "dietary protein*" OR [mh "Dietary Carbohydrates"] OR "dietary carbohydrate*" OR [mh "Dietary Fats"] OR "dietary fat*" OR hypocaloric OR hypocaloric) NEAR/6 (diet OR diets OR consumption OR intake OR supplement*))

\#3 - (([mh "Guideline Adherence"] OR guideline adherence*) NEAR/6 (diet OR dietary OR food OR beverage* OR nutrition $\left.{ }^{\star}\right)$ )

\#4 - ("diet score*" OR "diet quality score*" OR "diet quality index*" OR kidmed OR "diet index*" OR "dietary index" OR "food score*" OR MedDietScore OR "healthy eating index"):ti,ab,kw

\#5 - ((pattern OR patterns OR consumption OR habit*) NEAR/6 ([mh ^"Diet"] OR diet OR diets OR dietary OR [mh "Food"] OR food OR foods OR [mh "Beverages"] OR beverage OR beverages))

\section{\#6 - \#1 OR \#2 OR \#3 OR \#4 OR \#5}

\#7 - [mh "Breast Neoplasms"] OR [mh "Colorectal Neoplasms"] OR [mh "Intestinal Polyps"] OR [mh "Prostatic Neoplasms"] OR [mh "Lung Neoplasms"] OR [mh "Liver Neoplasms"] OR [mh "Pancreatic Neoplasms"] OR [mh "Endometrial Neoplasms"] OR [mh "Endometrial Hyperplasia"] OR [mh "Leukemia"]

\#8 - ("breast neoplasm*" OR "breast cancer*" OR "breast carcino*" OR "colorectal neoplasm*" OR "colorectal cancers*" OR "colorectal carcino*" OR "colon neoplasm*" OR "colon cancer*" OR "colon carcino*" OR "intestinal polyp*" OR "colonic polyp*" OR "colorectal polyp*" OR "colorectal lesion*" OR "rectal neoplasm*" OR "rectal cancer*" OR "rectal carcino*" OR "prostate neoplasm*" OR "prostate cancer*" OR "prostate carcino*" OR "lung neoplasm*" OR "lung carcino*" OR "lung cancer*" OR "liver neoplasm*" OR "liver cancer*" OR "hepatic neoplasm*" OR "hepatic cancer*" OR "hepatocellular carcino*" OR "pancreatic neoplasm*" OR "pancreatic cancer*" OR "pancreatic carcino*" OR "pancreatic adenocarcinoma*" OR "pancreatic neuro*" OR "ampullary cancer*" OR "ampullary carcino*" OR "exocrine cancer" OR "exocrine carcino*" OR "endometrial neoplasm*" OR "endometrial cancer*" OR "endometrial carcino*" OR "endometrioid neoplasm*" OR "endometrioid cancer*" OR "endometrioid carcino*" OR "endometrial hyperplasia*" OR leukem* OR leukaem* OR leucem* OR leucaem*):ti,ab,kw

\#9 - ((cancer* OR [mh "Neoplasms"] OR neoplasm* OR carcino* OR [mh "Carcinogens"] OR malignan* OR adenocarcinoma* OR sarcoma* OR metastasis OR metastases OR tumor OR tumors OR tumour* OR [mh "Polyps"] OR polyp OR polyps) NEAR/6 ([mh "Colon"] OR colonic* OR colon OR colorect* OR rectal OR rectum OR [mh ^"Colonic Diseases"] OR [mh "Breast"] OR [mh ^"Breast Diseases"] OR breast* OR mammary OR [mh "Prostate"] OR prostate* OR prostatic OR [mh ^"Prostatic Diseases"] OR [mh "Lung"] OR [mh ^"Lung Diseases"] OR lung OR pulmonary OR endometri* OR endometrium* OR [mh ^"Uterine Diseases"] OR "uterine diseases*" OR [mh "Liver"] OR liver OR [mh ^"Liver Diseases"] OR [mh "Pancreas"] OR pancreas OR pancreati* OR [mh ^"Pancreatic Diseases"] OR [mh "Ampulla of Vater"] OR "ampulla of vater")) 
\#10 - \#7 OR \#8 OR \#9

\#11 - \#6 AND \#10" with Publication Year from 2014 to 2020, in Trials (Word variations have been searched)

\section{Embase}

- Provider: Elsevier

- Date(s) Searched: January 13, 2020

- Date range searched: January 1, 2014-January 13, 2020

- Search Terms

\#1- 'feeding behavior'/de OR 'mediterranean diet'/exp OR 'dash diet'/exp OR 'gluten free diet'/exp OR 'paleolithic diet'/de OR 'vegetarian diet'/exp OR 'healthy diet'/exp OR 'western diet'/de OR 'low carbohydrate diet'/exp OR 'low fat diet'/de OR 'lipid diet'/exp OR 'protein diet'/exp OR 'protein restriction'/exp OR 'sodium restriction'/exp

\#2 - 'dietary pattern*':ab,ti OR 'diet pattern*':ab,ti OR 'eating pattern"':ab,ti OR 'food pattern' :ab,ti OR 'diet quality"':ab,ti OR 'eating habit':ab,ti OR 'dietary habit':ab,ti OR 'diet habit':ab,ti OR 'food habit*':ab,ti OR 'beverage habit':ab,ti OR 'feeding behavior':ab,ti OR 'dietary profile ${ }^{*}: a b, t i$ OR 'food profile ${ }^{*}: a b, t i$ OR 'diet profile':ab,ti OR 'eating profile*':ab,ti OR 'dietary guideline ${ }^{* \prime}: a b, t i$ OR 'dietary recommendation ${ }^{* \prime}: a b$, ti OR 'dietary intake ${ }^{* \prime}: a b, t i$ OR 'eating style $^{\star \prime}:$ ab,ti OR 'mediterranean diet $^{\star \prime}:$ ab,ti OR 'dietary approaches to stop hypertension $\operatorname{diet}^{* \prime}: a b, t i$ OR 'dash $\operatorname{diet}^{* \prime}: a b, t i$ OR 'gluten free $\operatorname{diet}^{* \prime}: a b, t i$ OR 'prudent diet' $\operatorname{diet}^{* \prime}: a b, t i$ OR 'vegetarian $\operatorname{diet}^{* \prime}: a b, t i$ OR 'vegan diet': ab,ti OR 'healthy diet':ab,ti OR 'plant based diet $^{* \prime}: a b, t i$ OR 'western diet $^{* \prime}: a b, t i$ OR 'low-carbohydrate diet':ab,ti OR 'high carbohydrate diet $^{* \prime}: a b$, ti OR 'ketogenic diet $^{* \prime}:$ ab,ti OR 'nordic diet ${ }^{* \prime}: a b$, ti OR 'high protein diet $^{* \prime}: a b, t i$ OR 'protein intake ${ }^{* \prime}: a b, t i$ OR 'high-fat diet ${ }^{* \prime}: a b$, ti OR 'low fat $\operatorname{diet}^{* \prime}: a b, t i$ OR 'low protein diet"':ab,ti OR 'low-sodium diet"':ab,ti OR 'low salt diet"':ab,ti

\#3 - ('dietary protein*' OR 'dietary carbohydrate*' OR 'dietary fat*' OR hypocaloric OR 'hypo caloric') NEAR/6 (diet OR diets OR consumption OR intake OR supplement*)):ab,ti

\#4 - ('guideline adherence*' NEAR/6 (diet OR dietary OR food OR beverage* OR nutrition*)):ab,ti

\#5 - 'diet score*':ab,ti OR 'diet quality score':ab,ti OR 'diet quality index'':ab,ti OR kidmed:ab,ti OR 'diet index"':ab,ti OR 'dietary index"':ab,ti OR 'food score*':ab,ti OR meddietscore:ab,ti OR 'healthy eating index*':ab,ti

\#6 - ((pattern OR patterns OR consumption OR habit*) NEAR/6 (diet OR diets OR dietary OR food OR foods OR beverage OR beverages)):ab,ti

\section{\#7 - \#1 OR \#2 OR \#3 OR \#4 OR \#5 OR \#6}

\#8 - 'breast cancer'/exp OR 'colorectal cancer'/exp OR 'intestine polyp'/exp OR 'prostate cancer'/exp OR 'lung cancer'/exp OR 'liver cancer'/exp OR 'pancreas cancer'/exp OR 'endometrium cancer'/exp OR 'endometrium hyperplasia'/exp OR 'leukemia'/exp

\#9 - 'breast neoplasm' 'ab,ti OR 'breast cancer':ab,ti OR 'breast carcino*':ab,ti OR 'colorectal neoplasm*':ab,ti OR 'colorectal cancers ${ }^{* 1}: a b, t i$ OR 'colorectal carcino*':ab,ti OR 'colon neoplasm*':ab,ti OR 'colon cancer':ab,ti OR 'colon carcino*':ab,ti OR 'intestinal polyp*':ab,ti OR 'colonic polyp*':ab,ti OR 'colorectal polyp*':ab,ti OR 'colorectal lesion*':ab,ti OR 'rectal neoplasm*':ab,ti OR 'rectal cancer*':ab,ti OR 'rectal carcino*':ab,ti OR 'prostate neoplasm':ab,ti 
OR 'prostate cancer"':ab,ti OR 'prostate carcino*':ab,ti OR 'lung neoplasm*':ab,ti OR 'lung carcino*':ab,ti OR 'lung cancer*':ab,ti OR 'liver neoplasm'*':ab,ti OR 'liver cancer':ab,ti OR 'hepatic neoplasm*':ab,ti OR 'hepatic cancer"':ab,ti OR 'hepatocellular carcino*':ab,ti OR 'pancreatic neoplasm ${ }^{* \prime}: a b, t i$ OR 'pancreatic cancer*':ab,ti OR 'pancreatic carcino*':ab,ti OR 'pancreatic adenocarcinoma ${ }^{* 1}: a b, t i$ OR 'pancreatic neuro"':ab,ti OR 'ampullary cancer': ab,ti OR 'ampullary carcino*':ab,ti OR 'exocrine cancer':ab,ti OR 'exocrine carcino*':ab,ti OR 'endometrial neoplasm*':ab,ti OR 'endometrial cancer":ab,ti OR 'endometrial carcino*':ab,ti OR 'endometrioid neoplasm*':ab,ti OR 'endometrioid cancer*':ab,ti OR 'endometrioid carcino*':ab,ti OR 'endometrial hyperplasia*':ab,ti OR leukem*:ab,ti OR leukaem*:ab,ti OR leucem*:ab,ti OR leucaem*:ab,ti

\#10 - ((cancer* OR neoplasm* OR carcino* OR malignan* OR adenocarcinoma* OR sarcoma* OR metastasis OR metastases OR tumor OR tumors OR tumour* OR polyp OR polyps) NEAR/6 (colonic* OR colon OR colorect* OR rectal OR rectum OR breast* OR mammary OR prostate* OR prostatic OR lung OR pulmonary OR endometri* OR endometrium* OR 'uterine diseases $^{* \prime}$ OR liver OR pancreas OR pancreati* OR 'ampulla of vater')):ab,ti

\#11 - \#8 OR \#9 OR \#10

\#12 - \#7 AND \#11

\#13 - \#7 AND \#11 AND [humans]/lim AND [english]/lim AND [2014-2020]/py NOT ([conference abstract]/lim OR [conference review]/lim OR [conference paper]/lim OR [editorial]/lim OR [erratum]/lim OR [letter]/lim OR [note]/lim OR [review]/lim OR [systematic review]/lim OR [meta analysis]/lim)

\section{Cumulative Index of Nursing and Allied Health Literature (CINAHL)}

- Provider: EBSCOhost

- Date(s) Searched: January 13, 2020

- Date range searched: January 1, 2014-January 13, 2020

- Search Strategy:

\#S1 - (MH "Eating Behavior") OR (MH "Mediterranean Diet") OR (MH "DASH Diet") OR (MH "Diet, Gluten-Free") OR (MH "Diet, High Protein") OR (MH "Diet, Ketogenic") OR (MH "Diet, Low Carbohydrate") OR (MH "Diet, Nordic") OR (MH "Diet, Paleolithic") OR (MH "Diet, Sodium-Restricted") OR (MH "Diet, Western") OR (MH "Vegetarianism") OR (MH "Diet, Atherogenic") OR (MH "Diet, Fat-Restricted")

\#S2 - ("dietary pattern*" OR "diet pattern*" OR "eating pattern*" OR "food pattern*" OR "diet quality*" OR "eating habit*" OR "dietary habit*" OR "diet habit" OR "food habit*" OR "beverage habit*" OR "feeding behavior" OR "dietary profile*" OR "food profile*" OR "diet profile*" OR "eating profile*" OR "dietary guideline*" OR "dietary recommendation*" OR "dietary intake*" OR "eating style*" OR "Mediterranean Diet" OR "Dietary Approaches To Stop Hypertension Diet" OR "DASH diet*" OR "Gluten Free diet" OR "prudent diet"" OR "Paleolithic Diet" OR "vegetarian diet" ${ }^{*}$ OR "vegan diet*" OR "healthy diet" OR "plant based diet*" OR "western diet $^{* "}$ OR "low-carbohydrate diet" OR "high carbohydrate diet*" OR "Ketogenic Diet*" OR "Nordic Diet*" OR "high protein diet" OR "protein intake*" OR "high-fat diet" OR "low fat diet" OR "low protein diet" OR "low-sodium diet" OR "low salt diet"

\#S3 - (((MH "Dietary Proteins+") OR "dietary protein*" OR (MH "Dietary Carbohydrates+") OR "dietary carbohydrate*" OR (MH "Dietary Fats+") OR "dietary fat*") N6 (diet OR diets OR 
consumption OR intake OR supplement $\left.{ }^{*}\right)$ )

\#S4 - ((MH "Guideline Adherence") OR “guideline adherence*”) N6 (diet OR dietary OR food OR beverage* OR nutrition*))

\#S5 - "diet score*" OR "diet quality score*" OR "diet quality index*" OR kidmed OR "diet index*" OR "dietary index*" OR "food score*" OR MedDietScore OR "healthy eating index*"

\#S6 - ((pattern OR patterns OR consumption OR habit*) N6 ((MH "Diet") OR diet OR diets OR dietary OR (MH "Food+") OR food OR foods OR (MH "Beverages+") OR beverage OR beverages))

\section{\#S7 - S1 OR S2 OR S3 OR S4 OR S5 OR S6}

\#S8 - (MH "Breast Neoplasms+") OR (MH "Colorectal Neoplasms+") OR (MH "Intestinal Polyps+") OR (MH "Prostatic Neoplasms+") OR (MH "Lung Neoplasms+") OR (MH "Liver Neoplasms+") OR (MH "Pancreatic Neoplasms+") OR (MH "Endometrial Neoplasms") OR (MH "Leukemia+")

\#S9 - "breast neoplasm*" OR "breast cancer*" OR "breast carcino*" OR "colorectal neoplasm*" OR "colorectal cancers*" OR "colorectal carcino*" OR "colon neoplasm*" OR "colon cancer*" OR "colon carcino*" OR "intestinal polyp*" OR "colonic polyp*" OR "colorectal polyp*" OR "colorectal lesion*" OR "rectal neoplasm*" OR "rectal cancer" OR "rectal carcino*" OR "prostate neoplasm*" OR "prostate cancer*" OR "prostate carcino*" OR "lung neoplasm*" OR "lung carcino*" OR "lung cancer*" OR "liver neoplasm*" OR "liver cancer*" OR "hepatic neoplasm*" OR "hepatic cancer*" OR "hepatocellular carcino*" OR "pancreatic neoplasm*" OR "pancreatic cancer*" OR "pancreatic carcino*" OR "pancreatic adenocarcinoma*" OR "pancreatic neuro*" OR "ampullary cancer*" OR "ampullary carcino*" OR "exocrine cancer*" OR "exocrine carcino*" OR "endometrial neoplasm*" OR "endometrial cancer*" OR "endometrial carcino*" OR "endometrioid neoplasm*" OR "endometrioid cancer" OR "endometrioid carcino*" OR "endometrial hyperplasia*" OR leukem* OR leukaem* OR leucem* OR leucaem*

\#S10 - ((cancer* OR (MH "Neoplasms+") OR neoplasm* OR carcino* OR (MH "Carcinogens+") OR malignan* OR adenocarcinoma* OR sarcoma* OR metastasis OR metastases OR tumor OR tumors OR tumour* OR (MH "Polyps+") OR polyp OR polyps) N6 (colonic* OR colon OR colorect* OR rectal OR rectum OR (MH "Colon+") OR (MH "Colonic Diseases") OR (MH "Breast+") OR (MH "Breast Diseases") OR breast* OR mammary OR (MH "Prostate") OR prostate* OR prostatic OR (MH "Prostatic Diseases") OR (MH "Lung+") OR (MH "Lung Diseases") OR lung OR pulmonary OR endometri* OR endometrium* OR (MH "Uterine Diseases") OR "uterine diseases*" OR (MH "Liver") OR liver OR (MH "Liver Diseases") OR (MH "Pancreas+") OR pancreas OR pancreati* OR (MH "Pancreatic Diseases") OR "ampulla of vater"))

\section{\#S11 - S8 OR S9 OR S10}

\#S12 - S7 AND S11 NOT (MH "Literature Review" OR MH "Meta Analysis" OR MH "Systematic Review" OR MH "News" OR MH "Retracted Publication" OR MH "Retraction of Publication") Limiters - Publication Year: 2014-2020; Peer Reviewed; English Language; Human 


\section{LITERATURE SEARCH AND SCREENING RESULTS}

The flow chart (Figure 2) below illustrates the literature search and screening results for articles in this update to existing systematic reviews. The results of the electronic database searches, after removal of duplicates, were screened independently by two NESR analysts using a step-wise process by reviewing titles, abstracts, and full-texts to determine which articles met the inclusion criteria. Refer to Table 15 for the rationale for exclusion for each excluded full-text article. A manual search was done to find articles that were not identified when searching the electronic databases; all manually identified articles were also screened to determine whether they meet criteria for inclusion. 
Figure 2: Flow chart of literature search and screening results

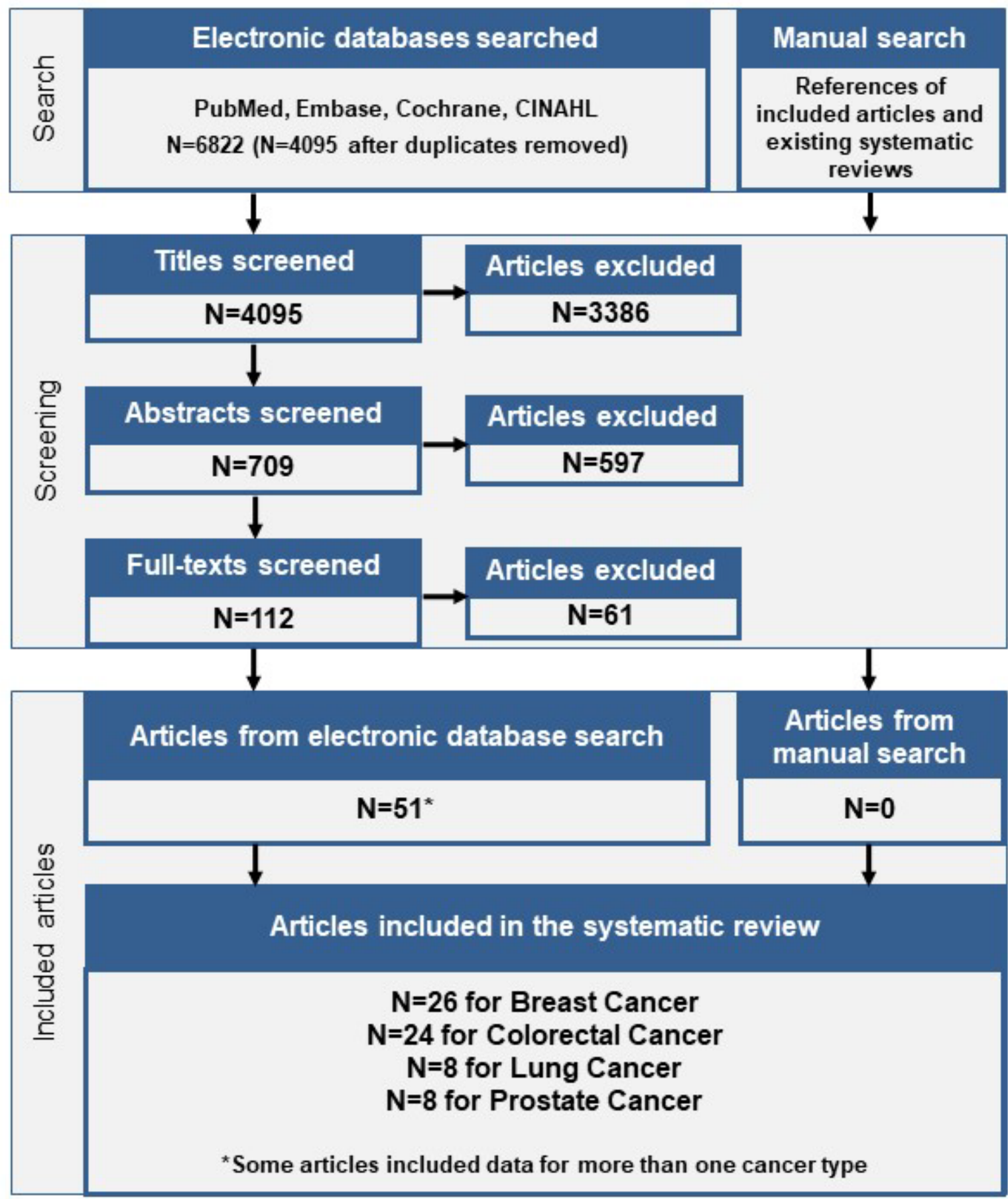




\section{Excluded articles from updated literature search}

The table below lists the articles excluded after full-text screening for this update to existing systematic reviews. At least one reason for exclusion is provided for each article, which may not reflect all possible reasons for exclusion. Information about articles excluded after title and abstract screening is available upon request.

\section{Table 16. Articles excluded after full-text screening with rationale for exclusion}

\section{Citation}

1. Mediterranean diet supplemented with extra virgin olive oil reduces the incidence of invasive breast cancer in a randomised controlled trial. Evidence-based medicine. 21 (2) (pp 72), 2016. Date of publication: April 2016.2016. doi:10.1136/ebmed-2015-110366

2. Akinyemiju, T, Wiener, H, Pisu, M. Cancer-related risk factors and incidence of major cancers by race, gender and region; analysis of the NIH-AARP diet and health study. BMC Cancer. 2017. 17:597. doi:10.1186/s12885-017-3557-1

3. Ax, E, Garmo, H, Grundmark, B, Bill-Axelson, A, Holmberg, L, Becker, W, Zethelius, B, Cederholm, T, Sjogren, P. Dietary patterns and prostate cancer risk: report from the population based ULSAM cohort study of Swedish men. Nutr Cancer. 2014. 66:77-87. doi:10.1080/01635581.2014.851712

4. Berberian, P, Obimba, C, Glickman-Simon, R, Sethi, T. Herbs for Low-Back Pain, Acupuncture for Psychological Distress, Osteopathic Manipulative Therapy for Chronic Migraine, Honey Dressings for Burns, Vegetarian Diet and Risk of Colorectal Cancer. Explore (NY). 2015. 11:410-4. doi:10.1016/j.explore.2015.07.011

5. Berstad, P, Botteri, E, Larsen, IK, Loberg, M, Kalager, M, Holme, O, Bretthauer, M, Hoff, G. Lifestyle changes at middle age and mortality: a population-based prospective cohort study. J Epidemiol Community Health. 2017. 71:5966. doi:10.1136/jech-2015-206760

6. Bradbury, KE, Murphy, N, Key, TJ. Diet and colorectal cancer in UK Biobank: a prospective study. Int J Epidemiol. 2019. doi:10.1093/ije/dyz064

7. Brinton, LA, Smith, L, Gierach, GL, Pfeiffer, RM, Nyante, SJ, Sherman, ME, Park, Y, Hollenbeck, AR, Dallal, CM. Breast cancer risk in older women: results from the NIH-AARP Diet and Health Study. Cancer Causes Control. 2014 25:843-57. doi:10.1007/s10552-014-0385-3

8. Castro-Quezada, I, Sanchez-Villegas, A, Martinez-Gonzalez, MA, Salas-Salvado, J, Corella, D, Estruch, R, Schroder, H, Alvarez-Perez, J, Ruiz-Lopez, MD, Artacho, R, Ros, E, Bullo, M, Sorli, JV, Fito, M, Ruiz-Gutierrez, V, Toledo, E, Buil-Cosiales, P, Garcia Rodriguez, A, Lapetra, J, Pinto, X, Salaverria, I, Tur, JA, Romaguera, D, Tresserra-Rimbau, A, Serra-Majem, L. Glycemic index, glycemic load and invasive breast cancer incidence in postmenopausal women: The PREDIMED study. Eur J Cancer Prev. 2016. 25:524-32. doi:10.1097/cej.0000000000000209

9. Catsburg, $\mathrm{C}$, Miller, AB, Rohan, TE. Adherence to cancer prevention guidelines and risk of breast cancer. Int $J$ Cancer. 2014. 135:2444-52. doi:10.1002/ijc.28887

10. Cifu, G, Arem, H. Adherence to lifestyle-related cancer prevention guidelines and breast cancer incidence and mortality. Ann Epidemiol. 2018. 28:767-773.e1. doi:10.1016/j.annepidem.2018.09.002

\section{Rationale}

Study design; Publication status

Intervention or exposure

Publication date

Study design; Publication status

Outcome

Intervention or exposure

Intervention or exposure

Intervention or exposure

Intervention or exposure

Intervention or exposure 


\section{Citation}

11. Dunneram, Y, Greenwood, DC, Cade, JE. Diet and risk of breast, endometrial and ovarian cancer: UK Women's Cohort Study. Br J Nutr. 2019. 122:564-574. doi:10.1017/s0007114518003665

12. Elwood, PC, Whitmarsh, A, Gallacher, J, Bayer, A, Adams, R, Heslop, L, Pickering, J, Morgan, G, Galante, J, Dolwani, S, Longley, M, Roberts, ZE. Healthy living and cancer: evidence from UK Biobank. Ecancermedicalscience. 2018. 12:792. doi:10.3332/ecancer.2018.792

13. Er, V, Lane, JA, Martin, RM, Emmett, P, Gilbert, R, Avery, KN, Walsh, E, Donovan, JL, Neal, DE, Hamdy, FC Jeffreys, M. Adherence to dietary and lifestyle recommendations and prostate cancer risk in the prostate testing for cancer and treatment (ProtecT) trial. Cancer Epidemiol Biomarkers Prev. 2014. 23:2066-77 . doi:10.1158/10559965.Epi-14-0322

14. Farvid, MS, Cho, E, Chen, WY, Eliassen, AH, Willett, WC. Adolescent meat intake and breast cancer risk. Int J Cancer. 2015. 136:1909-20. doi:10.1002/ijc.29218

15. Freedland, SJ. The effect of diet and supplements on prostate cancer. Clin Adv Hematol Oncol. 2014. 12:538-40.

16. Gilsing, AM, Schouten, LJ, Goldbohm, RA, Dagnelie, PC, van den Brandt, PA, Weijenberg, MP. Vegetarianism, low meat consumption and the risk of colorectal cancer in a population based cohort study. Sci Rep. 2015. 5:13484. doi:10.1038/srep13484

17. Gilsing, AM, Weijenberg, MP, Goldbohm, RA, Dagnelie, PC, van den Brandt, PA, Schouten, LJ. Vegetarianism, low meat consumption and the risk of lung, postmenopausal breast and prostate cancer in a population-based cohort study. Eur J Clin Nutr. 2016. 70:723-9. doi:10.1038/ejcn.2016.25

18. Guinter, MA, McLain, AC, Merchant, AT, Sandler, DP, Steck, SE. An estrogen-related lifestyle score is associated with risk of postmenopausal breast cancer in the PLCO cohort. Breast Cancer Res Treat. 2018. 170:613-622. doi:10.1007/s10549-018-4784-0

19. Haraldsdottir, A, Torfadottir, JE, Valdimarsdottir, UA, Adami, HO, Aspelund, T, Tryggvadottir, L, Thordardottir, M, Birgisdottir, BE, Harris, TB, Launer, LJ, Gudnason, V, Steingrimsdottir, L. Dietary habits in adolescence and midlife and risk of breast cancer in older women. PLoS One. 2018. 13:e0198017. doi:10.1371/journal.pone.0198017

20. Harris, HR, Bergkvist, L, Wolk, A. Adherence to the World Cancer Research Fund/American Institute for Cancer Research recommendations and breast cancer risk. Int J Cancer. 2016. 138:2657-64 . doi:10.1002/ijc.30015

21. He, X, Wu, K, Zhang, X, Nishihara, R, Cao, Y, Fuchs, CS, Giovannucci, EL, Ogino, S, Chan, AT, Song, M. Dietary intake of fiber, whole grains and risk of colorectal cancer: An updated analysis according to food sources, tumor location and molecular subtypes in two large US cohorts. Int J Cancer. 2019. 145:3040-3051. doi:10.1002/ijc.32382

22. Heitz, AE, Baumgartner, RN, Baumgartner, KB, Boone, SD. Healthy lifestyle impact on breast cancer-specific and allcause mortality. Breast Cancer Res Treat. 2018. 167:171-181. doi:10.1007/s10549-017-4467-2

23. Helander, S, Heinavaara, S, Sarkeala, T, Malila, N. Lifestyle in population-based colorectal cancer screening over 2year follow-up. Eur J Public Health. 2018. 28:333-338. doi:10.1093/eurpub/ckx139

\section{Rationale}

Intervention or exposure

Intervention or exposure

Intervention or exposure

Intervention or exposure

Study design; Publication status

Intervention or exposure

Intervention or exposure

Outcome

Intervention or exposure

Intervention or exposure

Intervention or exposure

Outcome 
24. Hidaka, BH, Kimler, BF, Fabian, CJ, Carlson, SE. An empirically derived dietary pattern associated with breast cancer risk is validated in a nested case-control cohort from a randomized primary prevention trial. Clin Nutr ESPEN. 2017. 17:8-17. doi:10.1016/j.clnesp.2016.10.008

25. Hirko, KA, Willett, WC, Hankinson, SE, Rosner, BA, Beck, AH, Tamimi, RM, Eliassen, AH. Healthy dietary patterns and risk of breast cancer by molecular subtype. Breast Cancer Res Treat. 2016. 155:579-88. doi:10.1007/s10549016-3706-2

26. Jones, P, Cade, JE, Evans, CEL, Hancock, N, Greenwood, DC. Does adherence to the World Cancer Research Fund/American Institute of Cancer Research cancer prevention guidelines reduce risk of colorectal cancer in the UK Women's Cohort Study?. Br J Nutr. 2018. 119:340-348. doi:10.1017/s0007114517003622

27. Kenfield, SA, DuPre, N, Richman, EL, Stampfer, MJ, Chan, JM, Giovannucci, EL. Mediterranean diet and prostate cancer risk and mortality in the Health Professionals Follow-up Study. Eur Urol. 2014. 65:887-94. doi:10.1016/j.eururo.2013.08.009

28. Key, TJ, Appleby, PN, Crowe, FL, Bradbury, KE, Schmidt, JA, Travis, RC. Cancer in British vegetarians: updated analyses of 4998 incident cancers in a cohort of 32,491 meat eaters, 8612 fish eaters, 18,298 vegetarians, and 2246 vegans. Am J Clin Nutr. 2014. 100 Suppl 1:378s-85s. doi:10.3945/ajcn.113.071266

29. Kohler, LN, Harris, RB, Oren, E, Roe, DJ, Lance, P, Jacobs, ET. Adherence to Nutrition and Physical Activity Cancer Prevention Guidelines and Development of Colorectal Adenoma. Nutrients. 2018. 10. doi:10.3390/nu10081098

30. Kouloulias, V, Platoni, K, Kantzou, I, Zygogianni, A, Kyriazi, K, Kougioumtzopoulou, A, Papaloucas, M, Papaloucas, C. Physical activity, early first delivery and residence as parameters for breast cancer prevention: an observational study. J buon. 2019. 24:1512-1515.

31. Lagerlund, M, Drake, I, Wirfalt, E, Sontrop, JM, Zackrisson, S. Health-related lifestyle factors and mammography screening attendance in a Swedish cohort study. Eur J Cancer Prev. 2015. 24:44-50. doi:10.1097/cej.0000000000000025

32. Lecuyer, L, Dalle, C, Lefevre-Arbogast, S, Micheau, P, Lyan, B, Rossary, A, Demidem, A, Petera, M, Lagree, M, Centeno, D, Galan, P, Hercberg, S, Samieri, C, Assi, N, Ferrari, P, Viallon, V, Deschasaux, M, Partula, V, Srour, B Latino-Martel, P, Kesse-Guyot, E, Druesne-Pecollo, N, Vasson, MP, Durand, S, Pujos-Guillot, E, Manach, C, Touvier M. Diet-Related Metabolomic Signature of Long-Term Breast Cancer Risk Using Penalized Regression: An Exploratory Study in the SU.VI.MAX Cohort. Cancer Epidemiol Biomarkers Prev. 2019. doi:10.1158/1055-9965.Epi$19-0900$

33. Liao, LM, Loftfield, E, Etemadi, A, Graubard, BI, Sinha, R. Substitution of dietary protein sources in relation to colorectal cancer risk in the NIH-AARP cohort study. Cancer Causes Control. 2019. 30:1127-1135. doi:10.1007/s10552-019-01210-1 

A, Song, M, Cao, Y, Twombly, TS, Shi, Y, Liu, H, Gu, M, Koh, H, Li, W, Du, C, Chen, Y, Li, C, Li, W, Mehta, RS, Wu,

K, Wang, M, Kostic, AD, Giannakis, M, Garrett, WS, Hutthenhower, C, Chan, AT, Fuchs, CS, Nishihara, R, Ogino, S, Giovannucci, EL. Diets That Promote Colon Inflammation Associate With Risk of Colorectal Carcinomas That Contain Fusobacterium nucleatum. Clin Gastroenterol Hepatol. 2018. 16:1622-1631.e3. doi:10.1016/j.cgh.2018.04.030

35. Llanos, AA, Krok, JL, Peng, J, Pennell, ML, Olivo-Marston, S, Vitolins, MZ, Degraffinreid, CR, Paskett, ED. Favorable effects of low-fat and low-carbohydrate dietary patterns on serum leptin, but not adiponectin, among overweight and obese premenopausal women: a randomized trial. Springerplus. 2014. 3:175. doi:10.1186/2193-1801-3-175

36. Makarem, N, Lin, Y, Bandera, EV, Jacques, PF, Parekh, N. Concordance with World Cancer Research Fund/American Institute for Cancer Research (WCRF/AICR) guidelines for cancer prevention and obesity-related cancer risk in the Framingham Offspring cohort (1991-2008). Cancer Causes Control. 2015. 26:277-286. doi:10.1007/s10552-014-0509-9

37. Marcondes, LH, Franco, OH, Ruiter, R, Ikram, MA, Mulder, M, Stricker, BH, Kiefte-de Jong, JC. Animal foods and postmenopausal breast cancer risk: a prospective cohort study. Br J Nutr. 2019. 122:583-591. doi:10.1017/s0007114519000072

38. McBride, D. Vegetarian Diets May Decrease Risk of Colorectal Cancer. ONS Connect. 2015. 30:51.

Outcome

39. Mehta, RS, Nishihara, R, Cao, Y, Song, M, Mima, K, Qian, ZR, Nowak, JA, Kosumi, K, Hamada, T, Masugi, Y, Bullman, S, Drew, DA, Kostic, AD, Fung, TT, Garrett, WS, Huttenhower, C, Wu, K, Meyerhardt, JA, Zhang, X, Willett, WC, Giovannucci, EL, Fuchs, CS, Chan, AT, Ogino, S. Association of Dietary Patterns With Risk of Colorectal Cancer Subtypes Classified by Fusobacterium nucleatum in Tumor Tissue. JAMA Oncol. 2017. 3:921-927. doi:10.1001/jamaoncol.2016.6374

40. Nguyen, S, Li, H, Yu, D, Gao, J, Gao, Y, Tran, H, Xiang, YB, Zheng, W, Shu, XO. Adherence to dietary recommendations and colorectal cancer risk: results from two prospective cohort studies. Int J Epidemiol. 2019. doi:10.1093/ije/dyz118

41. Nimptsch, K, Malik, VS, Fung, TT, Pischon, T, Hu, FB, Willett, WC, Fuchs, CS, Ogino, S, Chan, AT, Giovannucci, E, $\mathrm{Wu}, \mathrm{K}$. Dietary patterns during high school and risk of colorectal adenoma in a cohort of middle-aged women. Int $\mathrm{J}$ Cancer. 2014. 134:2458-67. doi:10.1002/ijc.28578

42. Papadimitriou, N, Muller, D, van den Brandt, PA, Geybels, M, Patel, CJ, Gunter, MJ, Lopez, DS, Key, TJ, PerezCornago, A, Ferrari, P, Vineis, P, Weiderpass, E, Boeing, H, Agudo, A, Sanchez, MJ, Overvad, K, Kuhn, T, Fortner, RT, Palli, D, Drake, I, Bjartell, A, Santiuste, C, Bueno-de-Mesquita, BH, Krogh, V, Tjonneland, A, Lauritzen, DF, Gurrea, AB, Quiros, JR, Stattin, P, Trichopoulou, A, Martimianaki, G, Karakatsani, A, Thysell, E, Johansson, I, Ricceri, F, Tumino, R, Larranaga, N, Khaw, KT, Riboli, E, Tzoulaki, I, Tsilidis, KK. A nutrient-wide association study for risk of prostate cancer in the European Prospective Investigation into Cancer and Nutrition and the Netherlands Cohort Study. Eur J Nutr. 2019. doi:10.1007/s00394-019-02132-z 
43. Park, YM, Steck, SE, Fung, TT, Merchant, AT, Elizabeth Hodgson, M, Keller, JA, Sandler, DP. Higher diet-dependent acid load is associated with risk of breast cancer: Findings from the sister study. Int J Cancer. 2019. 144:1834-1843.

Intervention or exposure doi:10.1002/ijc.31889

44. Printz, C. Vegetarian diet associated with lower risk of colorectal cancer. Cancer. 2015. 121:2667. doi:10.1002/cncr.29582

45. Rada-Fernandez de Jauregui, D, Evans, CEL, Jones, P, Greenwood, DC, Hancock, N, Cade, JE. Common dietary patterns and risk of cancers of the colon and rectum: Analysis from the United Kingdom Women's Cohort Study (UKWCS). Int J Cancer. 2018. 143:773-781. doi:10.1002/ijc.31362

46. Shivappa, N, Blair, CK, Prizment, AE, Jacobs, DR, Hebert, JR. Prospective study of the dietary inflammatory index and risk of breast cancer in postmenopausal women. Mol Nutr Food Res. 2017. 61. doi:10.1002/mnfr.201600592

47. Shivappa, N, Prizment, AE, Blair, CK, Jacobs, DR, Jr, Steck, SE, Hebert, JR. Dietary inflammatory index and risk of colorectal cancer in the lowa Women's Health Study. Cancer Epidemiol Biomarkers Prev. 2014. 23:2383-92. doi:10.1158/1055-9965.Epi-14-0537

48. Shivappa, N, Wang, R, Hebert, JR, Jin, A, Koh, WP, Yuan, JM. Association between inflammatory potential of diet and risk of lung cancer among smokers in a prospective study in Singapore. Eur J Nutr. 2019. 58:2755-2766 doi:10.1007/s00394-018-1825-8

49. Sieri, S. Consuming a high-fat diet is associated with increased risk of certain types of BC. J Natl Cancer Inst. 2014. 106. doi:10.1093/jnci/dju114

50. Sieri, S, Agnoli, C, Pala, V, Grioni, S, Brighenti, F, Pellegrini, N, Masala, G, Palli, D, Mattiello, A, Panico, S, Ricceri, F, Fasanelli, F, Frasca, G, Tumino, R, Krogh, V. Dietary glycemic index, glycemic load, and cancer risk: results from the EPIC-Italy study. Sci Rep. 2017. 7:9757. doi:10.1038/s41598-017-09498-2

51. Solbak, NM, Xu, JY, Vena, JE, Csizmadi, I, Whelan, HK, Robson, PJ. Diet quality is associated with reduced incidence of cancer and self-reported chronic disease: Observations from Alberta's Tomorrow Project. Preventive Medicine. 2017. 101:178-187. doi:10.1016/j.ypmed.2017.06.009

52. Sun, JW, Zheng, W, Li, HL, Gao, J, Yang, G, Gao, YT, Rothman, N, Lan, Q, Shu, XO, Xiang, YB. Dietary Glycemic Load, Glycemic Index, and Carbohydrate Intake on the Risk of Lung Cancer among Men and Women in Shanghai. Nutr Cancer. 2018. 70:671-677. doi:10.1080/01635581.2018.1460675

53. Tabung, FK, Steck, SE, Liese, AD, Zhang, J, Ma, Y, Caan, B, Chlebowski, RT, Freudenheim, JL, Hou, L, MossavarRahmani, Y, Shivappa, N, Vitolins, MZ, Wactawski-Wende, J, Ockene, JK, Hebert, JR. Association between dietary inflammatory potential and breast cancer incidence and death: results from the Women's Health Initiative. $\mathrm{Br} \mathrm{J}$ Cancer. 2016. 114:1277-85. doi:10.1038/bjc.2016.98

54. Tabung, FK, Steck, SE, Liese, AD, Zhang, J, Ma, Y, Johnson, KC, Lane, DS, Qi, L, Snetselaar, L, Vitolins, MZ, Ockene, JK, Hebert, JR. Patterns of change over time and history of the inflammatory potential of diet and risk of breast cancer among postmenopausal women. Breast Cancer Res Treat. 2016. 159:139-49. doi:10.1007/s10549016-3925-6 
55. $\quad$ Tabung, FK, Steck, SE, Ma, Y, Liese, AD, Zhang, J, Caan, B, Hou, L, Johnson, KC, Mossavar-Rahmani, Y, Shivappa, N, Wactawski-Wende, J, Ockene, JK, Hebert, JR. The association between dietary inflammatory index and risk of colorectal cancer among postmenopausal women: results from the Women's Health Initiative. Cancer Causes Control. 2015. 26:399-408. doi:10.1007/s10552-014-0515-y

56. Tabung, FK, Steck, SE, Ma, Y, Liese, AD, Zhang, J, Lane, DS, Ho, GYF, Hou, L, Snetselaar, L, Ockene, JK, Hebert, JR. Changes in the Inflammatory Potential of Diet Over Time and Risk of Colorectal Cancer in Postmenopausal Women. Am J Epidemiol. 2017. 186:514-523. doi:10.1093/aje/kwx115

57. Thomson, CA, McCullough, ML, Wertheim, BC, Chlebowski, RT, Martinez, ME, Stefanick, ML, Rohan, TE, Manson, JE, Tindle, HA, Ockene, J, Vitolins, MZ, Wactawski-Wende, J, Sarto, GE, Lane, DS, Neuhouser, ML. Nutrition and physical activity cancer prevention guidelines, cancer risk, and mortality in the women's health initiative. Cancer Prev Res (Phila). 2014. 7:42-53. doi:10.1158/1940-6207.Capr-13-0258

58. Urquiza-Salvat, N, Pascual-Geler, M, Lopez-Guarnido, O, Rodrigo, L, Martinez-Burgos, A, Cozar, JM, OcanaPeinado, FM, Alvarez-Cubero, MJ, Rivas, A. Adherence to Mediterranean diet and risk of prostate cancer. Aging Male. 2019. 22:102-108. doi:10.1080/13685538.2018.1450854

59. Wang, W, Fung, TT, Wang, M, Smith-Warner, SA, Giovannucci, EL, Tabung, FK. Association of the Insulinemic Potential of Diet and Lifestyle With Risk of Digestive System Cancers in Men and Women. JNCI Cancer Spectr. 2018. 2:pky080. doi:10.1093/jncics/pky080

60. Wang, Z, Uchida, K, Ohnaka, K, Morita, M, Toyomura, K, Kono, S, Ueki, T, Tanaka, M, Kakeji, Y, Maehara, Y, Okamura, T, Ikejiri, K, Futami, K, Maekawa, T, Yasunami, Y, Takenaka, K, Ichimiya, H, Terasaka, R. Sugars, sucrose and colorectal cancer risk: the Fukuoka colorectal cancer study. Scand J Gastroenterol. 2014. 49:581-8. doi:10.3109/00365521.2013.822091

61. Wie, GA, Cho, YA, Kang, HH, Ryu, KA, Yoo, MK, Kim, J, Shin, S, Chun, OK, Joung, H. Identification of major dietary patterns in Korean adults and their association with cancer risk in the Cancer Screening Examination Cohort. Eur J Clin Nutr. 2017. 71:1223-1229. doi:10.1038/ejcn.2017.6 